\title{
Exercise and training in NAFLD and insulin resistance
}

Citation for published version (APA):

Brouwers, B. (2016). Exercise and training in NAFLD and insulin resistance: psychological approach in humans . [Doctoral Thesis, Maastricht University]. Uitgeverij BOXPress || Proefschriftmaken.nl. https://doi.org/10.26481/dis.20160624bb

Document status and date:

Published: 01/01/2016

DOI:

10.26481/dis.20160624bb

Document Version:

Publisher's PDF, also known as Version of record

\section{Please check the document version of this publication:}

- A submitted manuscript is the version of the article upon submission and before peer-review. There can be important differences between the submitted version and the official published version of record.

People interested in the research are advised to contact the author for the final version of the publication, or visit the DOI to the publisher's website.

- The final author version and the galley proof are versions of the publication after peer review.

- The final published version features the final layout of the paper including the volume, issue and page numbers.

Link to publication

\footnotetext{
General rights rights.

- You may freely distribute the URL identifying the publication in the public portal. please follow below link for the End User Agreement:

www.umlib.nl/taverne-license

Take down policy

If you believe that this document breaches copyright please contact us at:

repository@maastrichtuniversity.nl

providing details and we will investigate your claim.
}

Copyright and moral rights for the publications made accessible in the public portal are retained by the authors and/or other copyright owners and it is a condition of accessing publications that users recognise and abide by the legal requirements associated with these

- Users may download and print one copy of any publication from the public portal for the purpose of private study or research.

- You may not further distribute the material or use it for any profit-making activity or commercial gain

If the publication is distributed under the terms of Article $25 \mathrm{fa}$ of the Dutch Copyright Act, indicated by the "Taverne" license above, 


\section{EXERCISE AND TRAINING}

IN NAFLD AND INSULIN

RESISTANCE

PHYSIOLOGICAL APPROACH IN HUMANS 
The studies described in this thesis were performed within NUTRIM School of Nutrition and Translational Research in Metabolism and are supported by the Dutch Diabetes Foundation (Diabetes Fonds Nederland, grant 2009.60.003).
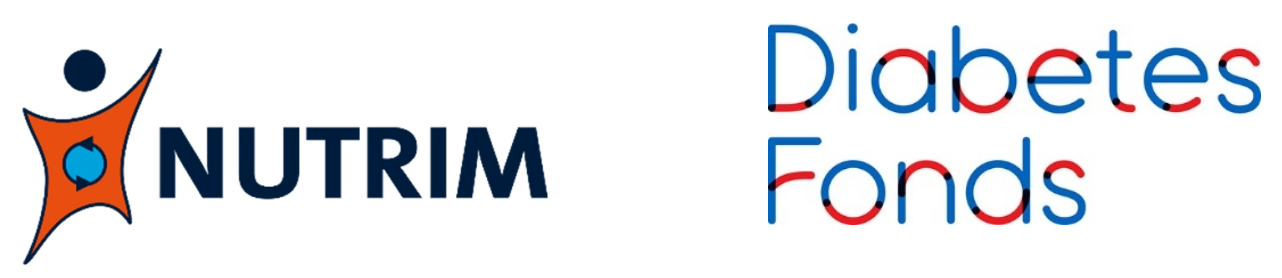

(C) Copyright Bram Brouwers 2016

Cover Design: Uitgeverij BOXPress || Proefschriftmaken.nl Lay Out: Bram Brouwers Printed by: Uitgeverij BOXPress || Proefschriftmaken.nl Published by: Uitgeverij BOXPress, Vianen 


\section{EXERCISE AND TRAINING \\ IN NAFLD AND INSULIN \\ RESISTANCE}

\section{PHYSIOLOGICAL APPROACH IN HUMANS}

\section{Proefschrift}

Ter verkrijging van de graad van doctor aan de Universiteit Maastricht, op gezag van de Rector Magnificus, Prof. Dr. L.L.G. Soete, volgens het besluit van het College van Decanen, in het openbaar te verdedigen op vrijdag 24 juni 2016 om 10.00 uur

door

Bram Brouwers

Geboren op 24 maart 1986 te Gent, België 


\section{Promotores}

Prof. dr. P. Schrauwen

Prof. dr. M.K.C. Hesselink

\section{Copromotor}

Dr. V.B. Schrauwen-Hinderling

\section{Beoordelingscommissie}

Prof. dr. Ir. A.M.W.J. Schols, voorzitter

Dr. M.R. Mensink, Wageningen Universiteit, Wageningen, Nederland Prof. dr. J. Plat

Prof. dr. C.D.A. Stehouwer

Prof. dr. S.R. Smith, Translational Research Institute for Metabolism and Diabetes and Sanford Burnham Prebys Medical Discovery Institute, Orlando, United States 


\section{TABLE OF CONTENT}

CHAPTER 1

GENERAL INTRODUCTION

CHAPTER 2

EFFECTS OF ACUTE EXERCISE AND EXERCISE TRAINING ON INTRAHEPATIC LIPID CONTENT IN HUMANS

CHAPTER 3

HEPATIC, SKELETAL MUSCLE AND ADIPOSE TISSUE INSULIN SENSITIVITY IS SIMILARLY IMPAIRED IN MEN WITH NAFL AND IN TYPE 2 DIABETIC PATIENTS

CHAPTER 4

ACUTE EXERCISE DOES NOT DECREASE LIVER FAT IN MEN WITH OVERWEIGHT OR NAFLD

CHAPTER 5 101

EXERCISE TRAINING REDUCES LIVER FAT CONTENT AND IMPROVES PERIPHERAL BUT NOT HEPATIC INSULIN SENSITIVITY IN OVERWEIGHT AND OBESE MEN WITH NON-ALCOHOLIC FATTY LIVER

CHAPTER 6

EXERCISE TRAINING-INDUCED EFFECTS ON ABDOMINAL SUBCUTANEOUS ADIPOSE TISSUE GENE EXPRESSION IN OBESE HUMANS

CHAPTER 7

151

COMBINED AEROBIC AND RESISTANCE EXERCISE TRAINING IMPROVES THE CARDIO-METABOLIC PROFILE IN OVERWEIGHT AND OBESE MEN WITH METABOLIC SYNDROME

CHAPTER 8

THE DUAL FACES OF EXERCISE TRAINING ON WHOLE BODY INSULIN SENSITIVITY

CHAPTER 9

GENERAL DISCUSSION 



\section{CHAPTER 1}

GENERAL INTRODUCTION 


\section{OBESITY AND TYPE 2 DIABETES MELLITUS}

The prevalence of obesity is very high and is reaching epidemic proportions worldwide ${ }^{1}$. Obesity is one of the most important risk factor contributing to mortality and the overall burden of disease worldwide ${ }^{2}$. The World Health Organization (WHO) describes obesity as one of the most blatantly visible, yet most neglected, public health problems that threatens to overwhelm both more and less developed countries ${ }^{3}$. Environmental factors like consumption of high-caloric diets and low levels of physical activity are likely to underlie this high prevalence of obesity. Since 1980, obesity prevalence has nearly doubled, affecting 1.4 billion adults in 2008. Moreover, more than 40 million children under the age of 5 suffered from obesity ${ }^{4}$. The problems of becoming obese are certainly not new. Already around 400 BCE Hippocrates noted adverse effects of excess adiposity. In his book On Airs, Waters, Places he even documented childhood obesity in the Scythian population, a nomadic race roaming around what is now $\operatorname{Iran}^{5}$. However, the development of obesity on the scale of a global population pandemic is certainly new ${ }^{5}$.

Obesity is associated with several disease conditions, the most devastating of which may be type 2 diabetes mellitus (T2DM) ${ }^{6}$. T2DM is characterized by a gradual reduction in insulin sensitivity over several years. Throughout this process the distinction is made between normal glucose tolerance (NGT), impaired glucose tolerance (IGT) and overt T2DM ${ }^{7}$. IGT is defined as an abnormal response to an oral glucose tolerance test ( $2 \mathrm{~h}$ oral glucose tolerance test (OGTT) plasma glucose values between 7.8 and $11.0 \mathrm{mmol} / \mathrm{I}^{8}$. The diagnosis of overt T2DM further includes fasting hyperglycemia (fasting plasma glucose $>6.1 \mathrm{mmol} / \mathrm{l}$ ), most commonly attributed to elevated hepatic glucose output due to resistance of the liver to the inhibitory effect of insulin on hepatic glucose production.

In 2011, the American Diabetes Association (ADA) reported that 25.8 million people in the United States were suffering from diabetes. Ninety percent of those people suffered from T2DM, the most common form of diabetes ${ }^{9,10}$. These numbers are likely to increase. Predictions by the WHO indicate that the worldwide prevalence of diabetes will be more than doubled between 2000 and $2030^{11}$. 


\section{INTRAHEPATIC LIPID CONTENT AND ITS LINK WITH INSULIN RESISTANCE}

Both obesity and T2DM are associated with insulin resistance ${ }^{12}$. The development of insulin resistance is often associated with the accumulation of fat within peripheral tissues, also known as ectopic fat accumulation ${ }^{13,14}$. In the liver, this is referred to as non-alcoholic fatty liver (NAFL), which includes patients with isolated steatosis and patients with steatosis and mild, nonspecific inflammation. NAFL is a subtype of non-alcoholic fatty liver disease (NAFLD), a spectrum of diseases which has been described for the first time in $1980^{15}$. NAFLD also includes the progressive subtype non-alcoholic steatohepatitis (NASH), which is distinguished from NAFL by the additional presence of features of hepatocellular injury with or without fibrosis $^{15-17}$.

Increased liver fat (intrahepatic lipid, IHL) content is present in up to $30 \%$ of the general adult population ${ }^{15,17,18}$. In obese people, the prevalence of NAFL may be as high as $50-70 \%{ }^{16-18}$. If alcoholic liver damage is not taken into account, then NAFL is nowadays the most abundant liver diseases worldwide and has a very high prevalence in North and South America, much of Asia-Pacific, the Middle East, and Europe. Because the prevalence of obesity and T2DM continued to rise since the $1980^{\prime} \mathrm{s}^{1,17}$, also the prevalence of NAFL will likely continue to rise.

The liver is one of the key sites of action of insulin. Moreover, the liver is one of the few and most important organs to release glucose to the circulation and hence plays a central role in glucose and lipid homeostasis. Thus, excessive fat storage in liver is a risk factor for the development of metabolic complications ${ }^{8}$. A strong association between IHL content and insulin resistance has been frequently reported ${ }^{19-26}$. Increased $\mathrm{IHL}$ content is not only associated with reduced hepatic insulin sensitivity ${ }^{22,23,25}$, but is also an independent risk factor to develop skeletal muscle and adipose tissue insulin resistance ${ }^{19,20}$. As a consequence, NAFL has been linked to the development of T2DM. Prospective studies revealed that elevations in liver enzymes predict the onset of T2DM later in life 27,28 , and the incidence of NAFL in T2DM might be as high as $70 \%{ }^{18,29}$. 
Interestingly, hepatic lipid accumulation can as well be cause as consequence of insulin resistance ${ }^{18}$ and several pathways can contribute to hepatic lipid accumulation. These pathways will we discussed in more detail in chapter $\mathbf{2}$.

\section{EXERCISE IN THE PREVENTION AND TREATMENT OF TYPE 2 DIABETES MELLITUS}

Exercise training is considered fundamental in the treatment and prevention of $\mathrm{T}^{2} \mathrm{DM}^{9}$. Exercise training - with an average follow-up of 2.8 years - delayed and reduced the incidence of T2DM in people at high risk by $58 \%^{30}$. Furthermore, exercise training can also modify lipid abnormalities and hypertension? Therapeutically, it is of interest to note that exercise training is at least as effective in older people as it is in younger individuals ${ }^{30}$. Current American Heart Association and ADA guidelines target $150 \mathrm{~min}$ of moderate-intensity or $60 \mathrm{~min}$ of vigorous physical activity per week to prevent and treat obesity-related diseases ${ }^{19}$. The American College of Sports Medicine suggests that people with T2DM should strive to achieve a minimum cumulative total of $1000 \mathrm{kcal}$ per week from physical activity $^{9}$. The physical activity programs should include endurance and resistance exercise, to develop and maintain cardiorespiratory fitness, body composition, muscle strength and endurance ${ }^{9}$.

\section{EXERCISE AND LIVER FAT METABOLISM}

In the past, most research has focused on the beneficial metabolic consequences of exercise training on skeletal muscle metabolism ${ }^{31-33}$, and less attention has been paid to the effects of exercise training on the liver. However, not all metabolic health effects of exercise training can be explained by skeletal muscle, suggesting that exercise training also has effects beyond muscle. Given the strong association between IHL content and insulin resistance and the liver's central role in glucose and lipid homeostasis, exercise might also have beneficial metabolic consequences on liver fat metabolism. 
One of the earliest observations of a possible link between regular physical activity and NAFL was in 1990 by Palmer et al. ${ }^{34}$. They report a normalization of alanine aminotransferase (ALAT) levels, a biomarker for liver disease, in overweight patients with NAFL who reduced body weight by $\geq 10 \%$ via combined dietary restriction and regular unstructured physical activity. However, ALAT is not a direct measure of hepatic fat content and to better understand the relationship between exercise and liver fat metabolism, it is important to measure IHL content directly, rather than rely on plasma markers of liver malfunction. The traditional gold standard method to measure $\mathrm{IHL}$ content is to assess the amount of triglycerides (TG) in a liver biopsy. Nowadays, however, proton magnetic resonance spectroscopy ( $\left({ }^{1} \mathrm{H}-\mathrm{MRS}\right)$ facilitates the quantification of $\mathrm{IHL}$ content non-invasively ${ }^{35}$. Since then, several studies have investigated the effect of exercise training ${ }^{36-43}$, but also acute exercise ${ }^{44-46}$, on hepatic lipid content. Nevertheless, the mechanism by which exercise affects liver fat content and metabolism in humans is still unresolved. Furthermore, consensus is lacking on whether putative positive effects of exercise on liver fat metabolism also results in improvements in hepatic insulin sensitivity, as reviewed in more detail in chapter 2 .

\section{OUTLINE OF THIS THESIS}

The research performed in this $\mathrm{PhD}$ thesis focusses on the impact exercise has as a therapeutic tool on modulating liver fat metabolism, insulin resistance and cardiovascular risk factors associated with T2DM.

In chapter 2, we review the contribution of possible pathways to hepatic lipid accumulation. Next, we give an overview of the current knowledge on how both acute exercise and exercise training might be effective in reducing $\mathrm{IHL}$ content.

Although most data indicate that a fatty liver promotes the vulnerability to progressive development of metabolic disturbances, the majority of these studies do not take into account obesity per se as a major contributing factor to the progressive metabolic aberrations. In chapter 3, we aimed to examine the metabolic disturbances related to insulin sensitivity in obese patients with or 
without NAFL, and compared the severity of these aberrations to BMI-matched patients with overt T2DM.

As outlined above, exercise training is an insulin sensitizing intervention and might also decrease IHL content. In chapter $\mathbf{4}$, we investigated if a single bout of exercise is able to lower intrahepatic lipid content in men with overweight or NAFL. In chapter 5, we examined if a well-controlled 12 week progressive, combined aerobic and resistance exercise training program results in reduced IHL content in healthy overweight/obese control subjects, subjects with NAFL and type 2 diabetic patients. We further investigated whether these possible reductions in IHL content upon exercise training coincide with improved hepatic insulin sensitivity.

In chapter 6, we examined the effect of a well-controlled 12 week progressive, combined aerobic and resistance exercise training program on adipose tissue in obese, well-phenotyped subjects. More specifically, we investigated if exercise training influenced abdominal subcutaneous adipose tissue gene expression of markers involved in mitochondrial biogenesis and function, browning, lipolysis, inflammation and adipokines.

The metabolic syndrome (MetS) is a constellation of cardiovascular risk factors associated with an increased risk for T2DM and cardiovascular disease. In chapter 7, the effect of a well-controlled 12 week progressive, combined aerobic and resistance exercise training program on risk components of the MetS in overweight/obese, middle-aged men is described. In addition, we investigated whether exercise training influences other risk factors of cardiovascular disease, such as central blood pressure, arterial stiffness and cardiac function.

Some people do not benefit from the well-known favorable effects of exercise training. Approximately $20 \%$ of individuals fail to improve glucose homeostasis, insulin sensitivity and/or muscle mitochondrial density. In a large cohort of people that performed 12 weeks of supervised exercise training, we investigated whether people who do not improve insulin sensitivity with exercise training also differently respond on exercise training regarding other metabolic benefits. These results are described in chapter 8. 
Finally, in chapter 9, the results of the previous chapters will be discussed in a broader perspective, in the light of the current knowledge and literature. Also future directions for research within the research field will be addressed. 


\section{REFERENCES}

1. Ng, M., et al. Global, regional, and national prevalence of overweight and obesity in children and adults during 1980-2013: a systematic analysis for the Global Burden of Disease Study 2013. Lancet 384, 766-781 (2014).

2. Haslam, D.W. \& James, W.P. Obesity. Lancet 366, 1197-1209 (2005).

3. Obesity: preventing and managing the global epidemic. Report of a WHO consultation. World Health Organ Tech Rep Ser 894, i-xii, 1-253 (2000).

4. Nguyen, T. \& Lau, D.C. The obesity epidemic and its impact on hypertension. Can J Cardiol 28, 326-333 (2012).

5. Haslam, D. \& Rigby, N. A long look at obesity. Lancet 376, 85-86 (2010).

6. Kahn, S.E., Hull, R.L. \& Utzschneider, K.M. Mechanisms linking obesity to insulin resistance and type 2 diabetes. Nature 444, 840-846 (2006).

7. Gastaldelli, A. Role of beta-cell dysfunction, ectopic fat accumulation and insulin resistance in the pathogenesis of type 2 diabetes mellitus. Diabetes Res Clin Pract 93 Suppl 1, S60-65 (2011).

8. Abdul-Ghani, M.A. \& DeFronzo, R.A. Pathophysiology of prediabetes. Curr Diab Rep 9, 193-199 (2009).

9. Albright, A., et al. American College of Sports Medicine position stand. Exercise and type 2 diabetes. Med Sci Sports Exerc 32, 1345-1360 (2000).

10. Association, A.D. Diagnosis and Classification of Diabetes Mellitus. Diabetes Care 34(2011).

11. Wild, S., Roglic, G., Green, A., Sicree, R. \& King, H. Global prevalence of diabetes: estimates for the year 2000 and projections for 2030. Diabetes Care 27, 1047-1053 (2004).

12. Reaven, G.M. Banting lecture 1988. Role of insulin resistance in human disease. Diabetes 37, 1595-1607 (1988).

13. Krssak, M. \& Roden, M. The role of lipid accumulation in liver and muscle for insulin resistance and type 2 diabetes mellitus in humans. Rev Endocr Metab Disord 5, 127-134 (2004).

14. Bilet, L., et al. Exercise-induced modulation of cardiac lipid content in healthy lean young men. Basic Res Cardiol 106, 307-315 (2011).

15. Rinella, M.E. Nonalcoholic fatty liver disease: a systematic review. Jama 313, 2263-2273 (2015). 
16. Lavoie, J.M. \& Gauthier, M.S. Regulation of fat metabolism in the liver: link to non-alcoholic hepatic steatosis and impact of physical exercise. Cell Mol Life Sci 63, 1393-1409 (2006).

17. Farrell, G.C. \& Larter, C.Z. Nonalcoholic fatty liver disease: from steatosis to cirrhosis. Hepatology 43, S99-S112 (2006).

18. Gaggini, M., et al. Non-alcoholic fatty liver disease (NAFLD) and its connection with insulin resistance, dyslipidemia, atherosclerosis and coronary heart disease. Nutrients 5, 1544-1560 (2013).

19. Utzschneider, K.M. \& Kahn, S.E. Review: The role of insulin resistance in nonalcoholic fatty liver disease. J Clin Endocrinol Metab 91, 4753-4761 (2006).

20. Korenblat, K.M., Fabbrini, E., Mohammed, B.S. \& Klein, S. Liver, muscle, and adipose tissue insulin action is directly related to intrahepatic triglyceride content in obese subjects. Gastroenterology 134, 1369-1375 (2008).

21. Yki-Jarvinen, $\mathrm{H}$. Liver fat in the pathogenesis of insulin resistance and type 2 diabetes. Dig Dis 28, 203-209 (2010).

22. Fabbrini, E., et al. Intrahepatic fat, not visceral fat, is linked with metabolic complications of obesity. Proc Natl Acad Sci U S A 106, 15430-15435 (2009).

23. Seppala-Lindroos, A., et al. Fat accumulation in the liver is associated with defects in insulin suppression of glucose production and serum free fatty acids independent of obesity in normal men. J Clin Endocrinol Metab 87, 3023-3028 (2002).

24. Kotronen, A., Juurinen, L., Tiikkainen, M., Vehkavaara, S. \& Yki-Jarvinen, H. Increased liver fat, impaired insulin clearance, and hepatic and adipose tissue insulin resistance in type 2 diabetes. Gastroenterology 135, 122-130 (2008).

25. Deivanayagam, S., et al. Nonalcoholic fatty liver disease is associated with hepatic and skeletal muscle insulin resistance in overweight adolescents. Am J Clin Nutr 88, 257-262 (2008).

26. Tiikkainen, M., et al. Liver-fat accumulation and insulin resistance in obese women with previous gestational diabetes. Obes Res 10, 859-867 (2002).

27. Fraser, A., Thinggaard, M., Christensen, K. \& Lawlor, D.A. Alanine aminotransferase, gamma-glutamyltransferase (GGT) and all-cause mortality: results from a population-based Danish twins study alanine 
aminotransferase, GGT and mortality in elderly twins. Liver Int 29, 14941499 (2009).

28. Andre, P., et al. Hepatic markers and development of type 2 diabetes in middle aged men and women: a three-year follow-up study. The D.E.S.I.R. Study (Data from an Epidemiological Study on the Insulin Resistance syndrome). Diabetes Metab 31, 542-550 (2005).

29. Targher, G., et al. Prevalence of nonalcoholic fatty liver disease and its association with cardiovascular disease among type 2 diabetic patients. Diabetes Care 30, 1212-1218 (2007).

30. Knowler, W.C., et al. Reduction in the incidence of type 2 diabetes with lifestyle intervention or metformin. N Engl J Med 346, 393-403 (2002).

31. Meex, R.C., et al. Restoration of muscle mitochondrial function and metabolic flexibility in type 2 diabetes by exercise training is paralleled by increased myocellular fat storage and improved insulin sensitivity. Diabetes 59, 572-579 (2010).

32. Bruce, C.R., Kriketos, A.D., Cooney, G.J. \& Hawley, J.A. Disassociation of muscle triglyceride content and insulin sensitivity after exercise training in patients with Type 2 diabetes. Diabetologia 47, 23-30 (2004).

33. Misra, A., et al. Effect of supervised progressive resistance-exercise training protocol on insulin sensitivity, glycemia, lipids, and body composition in Asian Indians with type 2 diabetes. Diabetes Care 31, 1282-1287 (2008).

34. Palmer, M. \& Schaffner, F. Effect of weight reduction on hepatic abnormalities in overweight patients. Gastroenterology 99, 1408-1413 (1990).

35. Reeder, S.B., Cruite, I., Hamilton, G. \& Sirlin, C.B. Quantitative assessment of liver fat with magnetic resonance imaging and spectroscopy. J Magn Reson Imaging 34, 729-749 (2011).

36. Bacchi, E., et al. Both resistance training and aerobic training reduce hepatic fat content in type 2 diabetic subjects with NAFLD (The RAED2 randomized trial). Hepatology (2013).

37. Johnson, N.A., et al. Aerobic exercise training reduces hepatic and visceral lipids in obese individuals without weight loss. Hepatology 50, 1105-1112 (2009). 
38. Hallsworth, K., et al. Resistance exercise reduces liver fat and its mediators in non-alcoholic fatty liver disease independent of weight loss. Gut 60, 1278-1283 (2011).

39. Pugh, C.J., et al. Exercise training reverses endothelial dysfunction in nonalcoholic fatty liver disease. Am J Physiol Heart Circ Physiol (2014).

40. Lee, S., et al. Aerobic exercise but not resistance exercise reduces intrahepatic lipid content and visceral fat and improves insulin sensitivity in obese adolescent girls: a randomized controlled trial. Am J Physiol Endocrinol Metab 305, E1222-1229 (2013).

41. Sullivan, S., Kirk, E.P., Mittendorfer, B., Patterson, B.W. \& Klein, S. Randomized trial of exercise effect on intrahepatic triglyceride content and lipid kinetics in nonalcoholic fatty liver disease. Hepatology 55, 1738-1745 (2012).

42. Lee, S., et al. Effects of aerobic versus resistance exercise without caloric restriction on abdominal fat, intrahepatic lipid, and insulin sensitivity in obese adolescent boys: a randomized, controlled trial. Diabetes $61,2787-$ 2795 (2012).

43. Finucane, F.M., et al. The effects of aerobic exercise on metabolic risk, insulin sensitivity and intrahepatic lipid in healthy older people from the Hertfordshire Cohort Study: a randomised controlled trial. Diabetologia 53, 624-631 (2010).

44. Johnson, N.A., et al. Effect of prolonged exercise and pre-exercise dietary manipulation on hepatic triglycerides in trained men. Eur J Appl Physiol 112, 1817-1825 (2012).

45. Bucher, J., et al. The effect of a single $2 \mathrm{~h}$ bout of aerobic exercise on ectopic lipids in skeletal muscle, liver and the myocardium. Diabetologia (2014).

46. Egger, A., et al. The effect of aerobic exercise on intrahepatocellular and intramyocellular lipids in healthy subjects. PLoS One 8, e70865 (2013). 



\section{CHAPTER 2}

\section{EFFECTS OF ACUTE EXERCISE AND EXERCISE}

TRAINING ON INTRAHEPATIC LIPID CONTENT IN HUMANS

Bram Brouwers, Matthijs K.C. Hesselink, Patrick Schrauwen, Vera B. SchrauwenHinderling

Submitted 


\section{ABSTRACT}

Non-alcoholic fatty liver (NAFL) is the most common liver disorder in western society. NAFL originates from increased delivery of lipids to the liver, decreased hepatic lipid oxidation, a decreased secretion of hepatic lipids to the circulation or a combination of these factors. If delivery of lipids to the liver outweighs the sum of hepatic lipid oxidation and secretion, intrahepatic lipid (IHL) content starts to increase and NAFL may develop. NAFL is closely related to obesity and insulin resistance and a fatty liver increases the vulnerability to develop type 2 diabetes mellitus (T2DM). Exercise training is a recognized cornerstone in the treatment and prevention of T2DM. A large body of literature has described the beneficial metabolic consequences of exercise training on skeletal muscle. Recent studies have started to investigate the effect of exercise training on liver metabolism, but data until now is still limited, however. In this review, we first describe the routes by which IHL content is modulated. Secondly, we review whether and how these contributing routes might be modulated by long-term exercise training. Thirdly, we will focus on the effects of acute exercise on hepatic lipid metabolism, since exercise also might affect hepatic metabolism in the physically active state. This will give insight in whether the effect of exercise training on IHL content could be explained by the accumulated effect of acute bouts of exercise, or whether adaptations might occur only after long-term exercise training. The primary focus of this review will be on observations made in humans. Where human data is missing, data obtained from well-accepted animal models will be used. 


\section{INTRAHEPATIC LIPID ACCUMULATION}

The high prevalence of obesity, affecting 1.4 billion adults worldwide in $2008^{1}$, has reached pandemic proportions ${ }^{2}$. Environmental factors like consumption of highcaloric diets and low levels of physical activity are likely to underlie this high prevalence of obesity. Associated with obesity is the excessive storage of lipids in skeletal muscle, heart and liver, known as so-called 'ectopic' fat accumulation ${ }^{3}$. In liver, this is diagnosed as non-alcoholic fatty liver (NAFL), if a fatty liver occurs in the absence of high alcohol consumption. In obese people, the prevalence of NAFL may be as high as $50-70 \%{ }^{4,5}$. In a subset of patients, NAFL may develop further in non-alcoholic steatohepatitis (NASH), cirrhosis or liver carcinoma ${ }^{6}$.

Although NAFL is thought to be essentially benign and to be fully reversible, it should be noted that NAFL strongly correlates with hepatic and whole body insulin resistance ${ }^{7-9}$. Insulin resistance is the earliest hallmark in the development of type 2 diabetes mellitus (T2DM) and the risk of developing T2DM is strongly increased in the presence of insulin resistance. Interestingly, intrahepatic lipid (IHL) accumulation can as well be cause as consequence of insulin resistance.

A major challenge in investigating the role of IHL content in metabolic disorders like obesity and T2DM is the poor accessibility of hepatic tissue, as liver biopsies are normally only taken upon clear clinical indication. Nowadays, non-invasive tools have become more and more accessible and important in the investigation of liver metabolism. Proton magnetic resonance spectroscopy $\left({ }^{1} \mathrm{H}-\mathrm{MRS}\right)$ has been accepted as the non-invasive gold standard to measure $\mathrm{IHL}$ content $\mathrm{t}^{10}$. This has facilitated $\mathrm{IHL}$ content quantification over a wide range of recent human intervention studies.

Hepatic lipid accumulation can occur due to changes in (i) free fatty acid plasma concentrations, (ii) lipids originating from a meal, (iii) de novo lipogenic activity, (iv) hepatic very low density lipoprotein export or (v) hepatic fat oxidation (figure 1). These routes and their possible link with IHL accumulation are discussed below. 


\section{FREE FATTY ACIDS}

The uptake of plasma free fatty acids (FFA) by the liver might depend on both the concentration of FFA in plasma and the capacity of the liver to take up FFA from the plasma. However, it was shown that hepatic FFA uptake is not regulated and, as a results, plasma FFA concentrations are directly related to the influx of FFA to the liver ${ }^{11,12}$. Thus, an elevation of plasma FFA levels, predominantly originating from elevated white adipose tissue lipolysis, might contribute to an increasing hepatic triglycerides (TG) depot. This has been described in a sophisticated isotope study by Donnelly et al. ${ }^{13}$, in which isotope infusion started 4 days prior to a liver biopsy. It was demonstrated that the serum FFA pool accounted for approximately $60 \%$ of hepatic TG content in the fasting state in patients with NAFL. Iozzo and colleagues ${ }^{14}$ showed that hepatic plasma FFA uptake tended to be increased in obesity, together with a trend for increased plasma FFA levels. Further, in obese subjects that were characterized by high hepatic lipid content, a 2-fold higher systemic fasting FFA turnover (determined by ${ }^{13} \mathrm{C}$ FFA rate of appearance during a constant infusion of ${ }^{13} \mathrm{C}_{4}$ palmitate) has been measured in comparison to $\mathrm{BMI}$-matched controls with low IHL content ${ }^{15}$. Therefore, high fasting FFA concentrations associated with obesity ${ }^{16}$ might contribute to elevated IHL content ${ }^{17}$.

It should be noted that not all studies find associations between fasting FFA levels and $\mathrm{IHL}^{18,19}$, and postprandial FFA concentrations might be of equal importance in relation to IHL content. In metabolically healthy subjects, insulin efficiently inhibits white adipose tissue lipolysis in the postprandial phase, leading to the welldocumented reduction in FFA after a meal ${ }^{20,21}$. If adipose tissue becomes insensitive to insulin, this drop in plasma FFA concentrations is blunted and circulatory FFA concentrations stay relatively high ${ }^{22}$. The higher plasma concentrations of FFA after a meal may contribute to hepatic fat accumulation. Indeed, a study that compared older, obese, NAFL subjects to young, lean control subjects showed that suppression of lipolysis during insulin stimulation $\left(40 \mathrm{mU} / \mathrm{m}^{2} / \mathrm{min}\right)$ was significantly hampered in the NAFL group ${ }^{23}$. Later, Holt et al. ${ }^{17}$ elegantly demonstrated that in obese subjects, the reduction in FFA concentrations during an oral glucose tolerance test (OGTT) was diminished when IHL content was high. Similarly, after $3 \mathrm{~h}$ and $4 \mathrm{~h}$ of insulin infusion $\left(0.3 \mathrm{mU} / \mathrm{kg} / \mathrm{min}\right.$, approximately $16.0-20.0 \mathrm{mU} / \mathrm{m}^{2} / \mathrm{min}$ for men with BMI $26 \mathrm{~kg} / \mathrm{m}^{2}$ ) plasma FFA concentrations decreased less in subjects 
with high IHL content ${ }^{18}$. These studies suggest an important role of postprandial FFA plasma concentrations in determining IHL.

A recent study using adipose tissue microdialysis investigated adipose tissue insulin resistance in relation to $\mathrm{IHL}$ content in more detail24. Using adipose tissue microdialysis, it was found that interstitial glycerol release in the fasted state, a direct measure of adipose tissue function, was not different between patients with NASH and control subjects. However, glycerol release upon insulin stimulation was higher in NASH patients, concomitant with higher plasma FFA levels. This indicates that insulin resistance of adipose tissue upon insulin stimulation may be an underlying factor of fatty liver disease.

\section{LIPIDS ORIGINATING FROM A MEAL}

Fat in the liver can also originate from a meal. Dietary TG are hydrolyzed in the intestinal lumen and FFA and monoacylglycerol (MAG) are taken up by enterocytes ${ }^{25}$. Enterocytes resynthesize these FFA and MAG back to TG and package them into chylomicron particles for secretion via the lymphatic system. Release and uptake of chylomicron-fatty acids occurs via the action of lipoprotein lipase (LPL), which is an enzyme located in the endothelium of adipose tissue and skeletal muscle ${ }^{26-29}$. The chylomicron remnants can then penetrate the fenestrations in the liver for receptor-mediated uptake into hepatocytes ${ }^{30-32}$. An alternative route how meal-derived fatty acids can reach the liver is via chylomicron-TG "spillover" into the serum FFA pool after incomplete lipolysis via $\mathrm{LPL}^{29,31}$. The regulation of postprandial hepatic fatty acid (FA) uptake is incompletely elucidated and various modulators of LPL, such as liver-produced apolipoproteins and angiopoietin-like proteins, are suggested to influence postprandial fat deposition in the liver ${ }^{28}$.

An overconsumption of dietary fat might lead to a postprandial oversupply of both, FFA and chylomicron remnants and may increase IHL content after a single meal ${ }^{33}$. Only one study measured the contribution of dietary fatty acids to hepatic TG content directly ${ }^{13}$. After consuming a diet with a typical composition (15 energy \% protein, 30 energy \% fat, and 55 energy \% carbohydrates) for 3 days in combination 
with isotope infusion, it was found that $15 \%$ of the hepatic TG pool in NAFL patients originated from dietary FA. Moreover, several studies have investigated the effect of dietary modulations on IHL content using iso-, hypo- and hypercaloric diet interventions. In general, consuming an isocaloric low-fat diet seems to decrease $\mathrm{IHL}$ content, while consuming an isocaloric high-fat diet seems to increase IHL content ${ }^{34-36}$. Westerbacka et al. ${ }^{36}$ found that when obese women consumed an isocaloric low-fat diet (16\% of total energy as fat) for 2 weeks, IHL content decreased with $20 \%$, while 2 weeks of a high-fat isocaloric diet (56 energy \% fat) increased IHL content by $35 \%$. These results were confirmed in overweight men ${ }^{35}$, where IHL content decreased by $13 \%$ and increased by $17 \%$ after consumption of an isocaloric low- (20 energy \% fat) or high-fat (55 energy \% fat) diet, respectively, for 3 weeks. Utzschneider et al. ${ }^{34}$ found a significant decrease in IHL content after 4 weeks of a low-fat isocaloric diet ( 25 energy \% fat), but found no change after 4 weeks of high-fat isocaloric diet ( 45 energy $\%$ fat). The discrepancy in result upon high-fat diet with the study of Westerbacka et al. and Van Herpen et al. may be related to the $10 \%$ lower contribution of fat to the total energy consumed. Nevertheless, together, these data suggest that in isocaloric conditions, the amount of dietary fat consumed may be an important contributor to IHL content.

Contrary to low- and high-fat isocaloric conditions, both low- and high-fat hypocaloric diets decrease IHL content, often accompanied by a reduction in body weight ${ }^{37}$. On the opposite, low- and high-fat hypercaloric diets markedly increase $\mathrm{IHL}$ content in subjects with steatosis and subjects with low IHL content ${ }^{37}$. Thus, in energy imbalance, it appears that the diet composition may be less important, and that energy intake might be the main determinant for changes in $\mathrm{IHL}$ content.

\section{DE NOVO LIPOGENESIS}

While de novo lipogenesis (DNL) was initially thought to account for only a small part of the hepatic TG content in humans, more recent studies revealed the opposite by showing that at least in patients with a fatty liver, DNL contributed up to one-fourth of the hepatic TG pool measured after an overnight fast ${ }^{13}$. The process of DNL is stimulated by insulin, which activates hepatic sterol regulatory element binding protein-1 (SREBP-1) ${ }^{38}$. SREBP-1 activates fatty acid synthase (FAS) and acetyl-COA carboxylase (ACC). FAS and ACC are two rate-limiting steps in 
hepatic fatty acid biosynthesis ${ }^{39,40}$, and lowering the activity of FAS and ACC will lower hepatic lipogenesis.

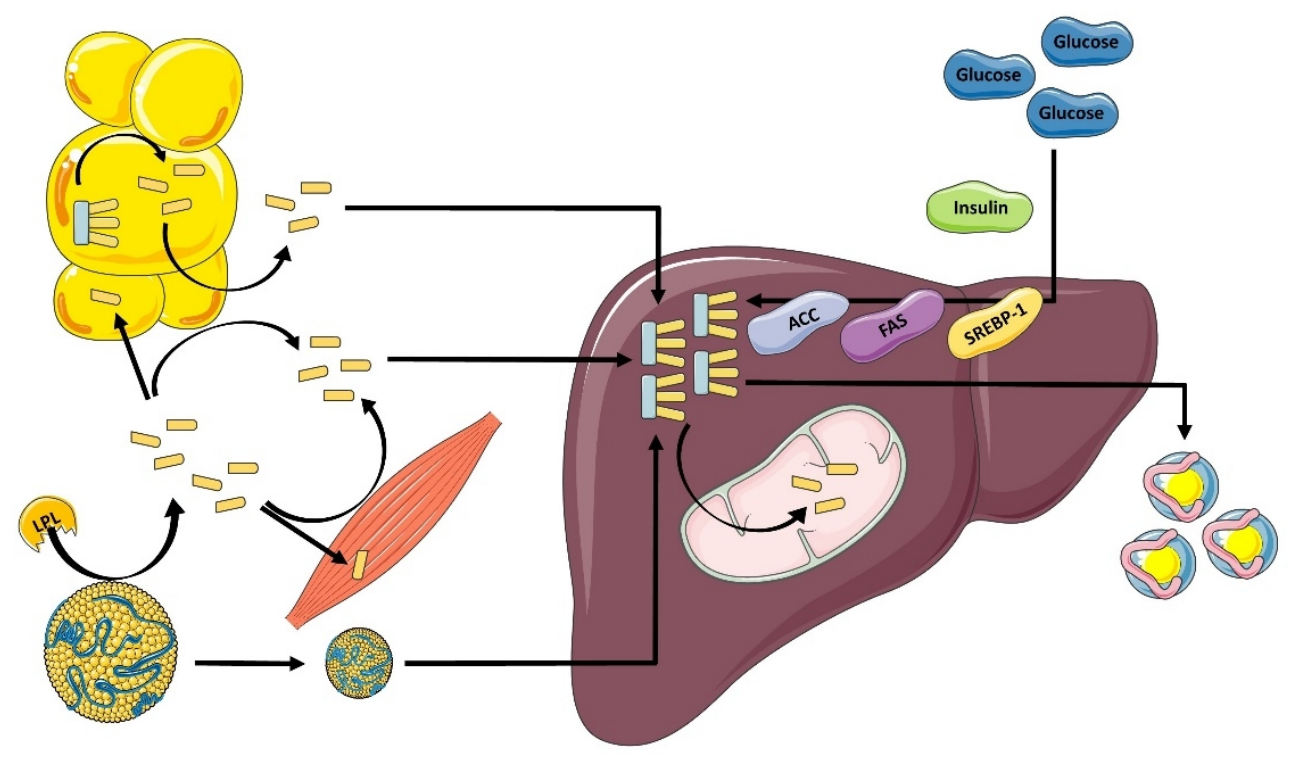

Figure 1. Pathways involved in hepatic lipid metabolism.

Adipose tissue releases free fatty acids (FFA) into the plasma via the process of lipolysis. Elevated fasting and postprandial plasma FFA, origination from reduced inhibition of adipose tissue lipolysis, are taken up at the hepatic site. Fat originating from a meal is transported in chylomicrons. Adipose tissue and skeletal muscle take up the chylomicronfatty acids (FA) via the action of lipoprotein lipase (LPL), while chylomicron remnants are taken up by the liver. However, when dietary fat availability is very high, the released FA end up in the plasma FFA pool via chylomicron-FA "spillover", and these FFA are taken up by the liver. Furthermore, hyperinsulinemia increases hepatic glucose uptake, which activates de novo lipogenesis via sterol regulatory element binding protein-1 (SREBP-1), fatty acid synthase (FAS) and acetyl-CoA carboxylase (ACC). To compensate for the increased hepatic fat delivery and synthesis, hepatic triglyceride secretion via VLDL and hepatic triglyceride mitochondrial oxidation are upregulated.

After consuming an isocaloric high-fat low-carbohydrate diet for 5 days (40 energy $\%$ fat and 46 energy \% carbohydrates), it was found that DNL was 5.3- and 3.7-fold higher in hyperinsulinemic obese subjects in comparison to more insulin sensitive lean and obese individuals ${ }^{41}$. Also in insulin resistant NAFL subjects DNL was significantly higher when compared to people with low IHL content ${ }^{42}$. It should be 
noted that these NAFL subjects were characterized by elevated fasting insulin levels. Insulin is a strong stimulator of lipogenesis and therefore, hyperinsulinemia may be underlying the increased rates of $D N L$, thereby contributing to hepatic lipid accumulation in humans.

\section{HEPATIC VLDL METABOLISM}

Rodents in which the very low density lipoprotein (VLDL) assembly is compromised, are the most commonly used dietary animal model to investigate NAFL ${ }^{43}$. These animals are fed a methionine- and choline-deficient (MCD) diet, which impairs hepatic VLDL secretion and in turn, the animals develop hepatic steatosis ${ }^{44}$. However, the value of such models could be questioned, as decreased VLDL production is generally not thought to be an underlying factor in the development of NAFL in the general population. An association between low VLDL production and increased fat storage has only been described in relation to rare apolipoprotein B-100 (apoB100) mutations ${ }^{45}$. On the contrary, in the general population, an elevated VLDL-TG secretion rate upon higher liver fat content was measured in many studies after an overnight fast ${ }^{7,42,46,47}$. In NAFL patients using stable isotope tracer, for example, a 2-fold higher hepatic VLDL-TG secretion rate compared to BMI-matched control subjects has been consistently demonstrated ${ }^{7,42,46}$. Also during the postprandial phase elevated VLDL-TG concentrations were observed in patients with NASH and in T2DM subjects ${ }^{48,49}$.

Despite consensus on the increased VLDL-TG secretion in NAFL, it is still unclear how hepatic lipid content determines VLDL secretion rate. While IHL content in obese subjects showed a positive, linear correlation with VLDL-TG secretion in one study $^{50}$, another study described a rise-to-plateau relationship between hepatic fat content and VLDL-TG secretion ${ }^{46}$. In the latter study, a direct correlation between IHL content and VLDL-TG secretion was observed among subjects with low IHL content, but not among subjects with NAFL. This may imply that there is an upper limit to VLDL-TG secretion, and that above this threshold VLDL-TG secretion rate does not further increase despite a progressive increase in IHL. This rise-to-plateau relationship might be explained by the limited capacity to increase VLDL-apoB100 production, which represents the number of VLDL particles secreted. Despite 
higher VLDL-TG secretion in NAFL patients, VLDL-apoB100 secretion was not different between people with elevated IHL content and people with normal IHL content ${ }^{46}$. Therefore, with elevated IHL content, the TG loading of VLDL particles increases until maximal size of the particles is reached and TG export saturation will be attained. After this, VLDL export cannot be matched with hepatic lipid storage anymore and hepatic lipid accumulation increases even further.

\section{HEPATIC MITOCHONDRIAL METABOLISM}

Oxidation of FA is the preferred hepatic energy source in the fasted state ${ }^{51}$ and the insulin resistant, fatty liver tends to activate hepatic oxidative metabolism ${ }^{14,23}$. The latter may be an adaptation to increased energy demand, as some endogenous pathways, such as gluconeogenesis, are upregulated with insulin resistance ${ }^{15}$. Also, ongoing liver damage during NAFL and concomitant hepatocellular regeneration may increase the hepatic energy requirements, as occurs in other forms of liver damage ${ }^{15,52}$.

Using positron emission tomography, a 2-fold higher hepatic oxidation of FA was measured in obese versus non-obese humans ${ }^{14}$. When subjects with and without NAFL were investigated, hepatic mitochondrial oxidative metabolism was doubled in NAFL subjects and mitochondrial oxidative metabolism was positively correlated with $\mathrm{IHL}$ content ${ }^{15}$. Koliaki et al. ${ }^{53}$ further measured mitochondrial function in isolated mitochondria of liver biopsies. In their study, maximal coupled (state 3) and uncoupled (state $u$ ) mitochondrial respiration was higher in obese subjects with and without NAFL than in lean control subjects, whereas mitochondrial content was comparable between all groups. Thus, hepatic steatosis develops in the presence of elevated mitochondrial oxidative metabolism in the liver. This suggests that the increase in oxidative metabolism (and in VLDL-TG secretion, as mentioned previously) cannot fully compensate for increased lipid delivery, and the excess lipids are redirected towards hepatic TG storage. Although mitochondrial content was comparable between NAFL and obese control subjects, Koliaki et al. ${ }^{53}$ found that the mRNA expression of proliferator-activated receptor gamma coactivator 1-alpha (PGC-1 $\alpha$ ), nuclear respiratory factor-1 (NRF1) and mitochondrial transcriptional factor A (TFAM) was markedly reduced in NAFL 
subjects in comparison to obese control subjects, suggesting lower hepatic mitochondrial biogenesis.

The reductions in mRNA levels of key intermediates in mitochondrial biogenesis may pave the way to further NAFL progression, and eventually may lead to impaired mitochondrial function. This hypothesis is supported by the decreased mitochondrial function observed in $\mathrm{NASH}^{53-56}$. Impaired mitochondrial function in NASH has been demonstrated using an intravenous fructose bolus, which is known to deplete hepatic ATP concentrations followed by measuring hepatic ATP recovery, a well-known measurement for hepatic mitochondrial function ${ }^{55}$. Upon challenging patients with NASH with an intravenous fructose bolus ${ }^{54}$, ATP was comparably depleted in both control subjects and NASH patients immediately after fructose ingestion. However, $60 \mathrm{~min}$ after the fructose challenge, NASH patients barely recovered their hepatic ATP levels, whereas control subjects replenished their ATP stores back to baseline values ${ }^{54}$. Although this design did not permit to identify mitochondrial mechanisms responsible for delayed ATP recovery, a liverbiopsy study revealed a lower activity of all mitochondrial respiratory chain complexes in NASH patients $5^{56}$. In support of this data, Koliaki et al..$^{53}$ found that subjects with NASH had impaired maximal coupled and uncoupled mitochondrial respiration, which was accompanied by higher mitochondrial uncoupling, lipid peroxidation and reactive oxygen species (ROS) production as the anti-oxidant defense system was altered. Also PGC-1 $\alpha$ mRNA expression was decreased. Thus, the observed higher oxidative metabolism found with NAFL is lost with NASH, and reduced mitochondrial biogenesis may be important. 


\section{EFFECTS OF EXERCISE TRAINING ON LIVER FAT METABOLISM}

Exercise training is a recognized cornerstone in the treatment of obesity and its comorbidities, and recent studies have shown that exercise training might also have beneficial effects on IHL content ${ }^{57-68}$. One of the earliest observations of a possible link between regular physical activity and NAFL has been made by Palmer et al. ${ }^{69}$, who noted normalization of alanine aminotransferase (ALAT) plasma levels in overweight patients with NAFL who reduced body weight by $\geq 10 \%$ via combined dietary restriction and regular unstructured physical activity. After this, other studies showed that physical activity levels were correlated negatively with the prevalence of NAFL ${ }^{57-59}$. However, only few studies have investigated the direct effect of exercise training on IHL content in interventional studies ${ }^{60-68}$.

In obese, older subjects, 12 weeks of supervised aerobic exercise training for $1 \mathrm{~h}$ a day decreased IHL content significantly compared to a non-exercising control group ${ }^{61}$. Consistently, in obese, sedentary adults, 16 weeks of aerobic exercise training for 5 times a week, decreased IHL content with $10 \%$ in the absence of weight loss ${ }^{67}$, and even 4 weeks of aerobic exercise training positively affected IHL content in a comparable group of subjects ${ }^{63}$. Also resistance exercise training for 8 weeks lowered IHL content with $13 \%$ in obese, sedentary adults ${ }^{62}$. In the study of Bacchi et $a l .{ }^{60}$, it was demonstrated that both aerobic training and resistance training decreased IHL content to a similar extent (25 - $30 \%$ decrease from baseline). Van der Heijden et al. ${ }^{68}$ showed that 12 weeks of aerobic exercise training was also effective in lowering $\mathrm{IHL}$ content in younger people, reporting a decrease in IHL content from 8.9 to $5.6 \%$ in obese Hispanic adolescents. Interestingly, at least during adolescence, there also seems to be a gender effect. In the studies performed by Lee et al. ${ }^{64,65}$, adolescent boys showed decreased IHL content after both aerobic and resistance exercise ${ }^{64}$, while girls only benefited from aerobic exercise training ${ }^{65}$.

While the finding that exercise training reduces IHL content is very reproducible, the mechanisms by which exercise training affects liver fat content in humans are still unresolved. The simplest view is that the increase in energy expenditure due to 
physical activity simply induces a negative energy balance, which in turn may result in the mobilization of hepatic lipids as a substrate to fuel the energy deficit. Most exercise training studies, however, do not show significant effects on body mass ${ }^{61-}$ ${ }^{63,67}$ or fat and fat free mass ${ }^{61,62,67}$. In fact, only Bacchi et al. ${ }^{60}$ concluded that there was a significant reduction in $\mathrm{BMI}$ and fat mass upon exercise training. In this 4 month supervised aerobic or resistance exercise training program, T2DM patients with NAFL performed 3 times a week $60 \mathrm{~min}$ of aerobic exercise training (60 - $65 \%$ of heart rate reserve) or 3 times a week resistance exercise training focusing on 9 major muscle groups ( $70-80 \%$ of the 1 repeated maximum). Two months before onset of the training program, participants met with a nutritionist to encourage to follow a healthy diet, and this might have affected the observations. Caloric intake was measured by a 3-day food recall just before and at the end of the exercise training intervention. After exercise training, caloric intake decreased with 77.4 $\mathrm{kcal} /$ day in the aerobic training group and $91.9 \mathrm{kcal} /$ day in the resistance training group. Therefore, the decrease in caloric intake throughout the exercise training program might have been responsible for reduced BMI and fat mass, rather than the exercise training program itself.

As outlined above, NAFL develops in the presence of alterations in fat delivery, synthesis, secretion and oxidation. Since exercise training has the potential to lower IHL content, it may possibly do so by affecting one or more of the pathways involved.

\section{EFFECTS OF EXERCISE TRAINING ON FREE FATTY ACIDS}

Endurance-trained subjects are characterized by low fasting plasma FFA concentrations ${ }^{70}$, suggesting that regular exercise may have an effect on adipose tissue lipid FFA uptake and lipolysis. In line with this, a 6 month hypocaloric diet in combination with exercise resulted in decreased in vitro basal adipocyte lipolysis in obese, postmenopausal women ${ }^{71}$. Shojaee-Moradie and colleagues ${ }^{72}$ reported a decreased basal glycerol rate of appearance $\left(R_{a}\right)$ and palmitate $R_{a}$ (indicative of a lower rate of lipolysis) $72 \mathrm{~h}$ after a 6 week exercise training program in sedentary overweight men, however, this was not accompanied by a reduction in IHL content. Other studies did not observe exercise training induced effects on adipose tissue 
lipolysis. For example, using adipose tissue microdialysis in seventeen healthy elderly women, interstitial glycerol levels were not affected after 12 weeks of exercise training ${ }^{73}$. In studies that report a decrease in adipose tissue lipolysis, this is often paralleled by a concomitant decrease in (visceral) adipose tissue ${ }^{71,72}$, which may explain part of the effect on plasma FFA concentrations.

The exercise training induced decrease in hepatic fat content does not necessarily occur in parallel to decreased plasma FFA concentrations in the fasted state, as several studies report a decrease in hepatic fat content even though fasted plasma FFA concentrations remained unchanged ${ }^{62,67}$. Exercise training also promotes whole body insulin sensitivity and hence might also promote the insulin-stimulated suppression of adipose tissue lipolysis ${ }^{74}$. We previously found that 12 weeks of combined aerobic and resistance exercise training improved insulin-mediated suppression of plasma FFA both in T2DM subjects and BMI-matched healthy control subjects $^{75}$. Similarly, in obese men, 12 weeks of dynamic strength training did improve insulin-stimulated suppression of FFA ${ }^{76}$. Nevertheless, absolute FFA levels during insulin infusion were comparable with pre-intervention concentrations, and the effect was mainly due to an increase in fasting FFA levels after exercise training. In contrast, no change in insulin-stimulated suppression of FFA was observed after 12 weeks of aerobic exercise training in people with impaired glucose tolerance ${ }^{77,78}$ and/or impaired fasting glucose $\mathrm{e}^{77}$. In non-obese sedentary women insulinstimulated FFA suppression improved after 9 months of high intensity (80\% $\left.\mathrm{VO}_{2 \text { max }}\right)$, but not after moderate-intensity $\left(65 \% \mathrm{VO}_{2 \max }\right)$, aerobic exercise training ${ }^{79}$.

Next to the potential effect on adipose tissue lipolysis, exercise training may also favor the clearance of FA by skeletal muscle. In that respect, it is well established that endurance training improves whole body fat oxidation ${ }^{73,80}$ and this is accompanied by higher FA uptake in skeletal muscle. With exercise training, this increased capacity for FA uptake into the myocyte has been associated with the upregulation of membrane-associated FA transport proteins ${ }^{81}$. Using $14(\mathrm{R}, \mathrm{S})-\left[{ }^{18} \mathrm{~F}\right]-$ 6-thia-heptadecanoic $\left({ }^{18} \mathrm{~F}-\mathrm{FTHA}\right)$ acid (a FFA analog that is transported across the cellular membrane but is not further metabolized) during a hyperinsulinemiceuglycemic clamp, athletes possessed a higher FFA uptake in skeletal muscle, while hepatic retention of FFA was shown to be $20 \%$ lower than in healthy sedentary subjects $^{82}$. Moreover, using the same FFA analog in a monozygotic twin study, 
hepatic FFA uptake was $33 \%$ lower in the more active twin in comparison to the less active twin during a low-intensity $90 \mathrm{~min}$ knee extension exercise ${ }^{83}$.

Taken together, exercise training may lower IHL content via effects on plasma FFA. Whereas the effects on adipose tissue lipolysis are less clear, exercise training does seem to improve FA uptake in muscle. This might lower the FA availability for the liver, lowering hepatic FA uptake, and changing fatty acid partitioning from liver to muscle.

\section{EFFECTS OF EXERCISE TRAINING ON LIPIDS ORIGINATING FROM A MEAL}

Next to effects on plasma FFA levels, exercise training may also affect postprandial lipid metabolism. It is known that exercise training lowers the serum TG response to a fatty meal ${ }^{84}$ and the prevalence of postprandial hypertriglyceridemia is significantly higher in sedentary people compared to trained people ${ }^{85}$. The reduction in postprandial hypertriglyceridemia associated with endurance exercise adaptations is due to a decrease in chylomicron-TG half-life ${ }^{86}$. The difference in TG half-life between athletes and sedentary men is most probably due to a direct effect of exercise training on the lipoprotein lipase (LPL)-mediated TG removal to skeletal muscle ${ }^{86}$.

Changes in the activity of LPL may play a major role in the more favorable lipoprotein-lipid profile of physically active individuals. Heparin is known to release LPL from its endothelial binding sites into the circulating blood ${ }^{87}$ and post-heparin plasma LPL activity has been reported to be higher in endurance-trained individuals compared to inactive controls ${ }^{88,89}$. Furthermore, post-heparin LPL activity increased after 20 weeks of endurance training in healthy, sedentary subjects ${ }^{90}$. It is known that, following endurance training, LPL activity measured shortly after heparin infusion reflects LPL derived from skeletal muscle rather than from adipose tissue ${ }^{91}$. This suggests that with exercise training postprandial TG are more likely to be taken up by the skeletal muscle. 
Hepatic lipase $(\mathrm{HL})$ plays an important role in lipoprotein remodeling ${ }^{90}$, hydrolyzing TG and phospholipids from chylomicrons, high-, intermediate-, and low-density lipoproteins ${ }^{92,93}$, so that TG are taken up by the liver. It was shown that post-heparin $\mathrm{HL}$ activity reduced significantly after 20 weeks of endurance training ${ }^{90}$, and this might result in lower postprandial hepatic TG uptake. Although these indirect measurements give an indication for postprandial TG channeling towards skeletal muscle with exercise training and away from the liver, the exercise training effects on postprandial hepatic fat storage have not been investigated directly.

\section{EFFECTS OF EXERCISE TRAINING ON DE NOVO LIPOGENESIS}

The effect of exercise training on DNL in humans has not been investigated yet. However, it is well documented that plasma concentrations of insulin and glucose are lower in endurance-trained subjects ${ }^{94}$, as whole body insulin sensitivity is known to be improved ${ }^{70}$. Insulin is recognized to stimulate de novo lipogenesis from glucose $^{38}$. Lowered plasma concentrations of insulin and glucose may therefore blunt DNL. This holds true for the fasted situation but even more so for the metabolic response to a meal. In exercise training studies that lowered IHL content, decreases in fasting insulin ${ }^{61,64,65,68,95}$, fasting glucose ${ }^{62,66}, 2 \mathrm{~h}$ glucose $\mathrm{e}^{61,65,95}$ and $2 \mathrm{~h}$ insulin ${ }^{61,65,95}$ have been reported.

Whereas human data is very limited, the effect of exercise training on DNL has been studied in animal models. In Otsuka Long-Evans Tokushima Fatty (OLETF) rats, a rat model for T2DM, exercise training reduced ${ }^{96,97}$ or prevented ${ }^{98,99}$ hepatic fat storage concomitant with a decrease in key intermediates in hepatic fatty acid synthesis. Thus, in comparison to their sedentary littermates, OLETF rats performing voluntary exercise or exercise training for several weeks were characterized by a significant reduction in total SREBP-1C, ACC and FAS protein content and a marked increase in ACC phosphorylation, the inactive form. Decreases of key intermediates in hepatic DNL were accompanied by exercise training induced decreases in plasma glucose and insulin concentrations ${ }^{96,97,99}$, pointing to improved insulin sensitivity of the OLETF rats. Moreover, when trained OLETF rats were put back on a sedentary program for 4 weeks, after 12 weeks of voluntary wheel running, the exercise training induced suppression of lipogenesis-stimulating proteins and plasma 
glucose was partly maintained, together with a partly continued suppression of hepatic TG content ${ }^{99}$. These findings suggest that, at least in OLETF rats, exercise training leads to a sustained reduction of hepatic lipogenesis, which might be important in a continued suppression of IHL content.

\section{EFFECTS OF EXERCISE TRAINING ON HEPATIC VLDL METABOLISM}

Although VLDL metabolism is generally not thought to be an underlying factor in the development of fatty liver disease in the general population, exercise training could still influence hepatic lipid content by modifying VLDL metabolism. Until now, no study has investigated the effect of exercise training induced changes in VLDL metabolism on IHL content in humans, but some studies have investigated how exercise training influences the VLDL secretion and clearance in humans. For example, 6 months of supervised exercise training resulted in a significant decrease in VLDL-apoB100 secretion rate in obese, T2DM patients ${ }^{100}$. The VLDL-apoB100 catabolic rate, representing removal of VLDL particles from the vascular compartment by complete hydrolysis to intermediate-density lipoproteins (IDL) or by direct removal via the hepatic VLDL receptor ${ }^{100,101}$, did not change. Moreover, the VLDL-TG/apoB100 ratio was not altered, suggesting that there was no change in TG content per VLDL particle secreted. Since VLDL-apoB100 secretion rate decreased, plasma VLDL-TG concentrations decreased. These findings were confirmed in a 2 month exercise training experiment with sedentary, non-obese young men ${ }^{102}$. Stable isotope-labeled $\left[1,1,2,3,3,-{ }^{2} \mathrm{H}_{5}\right]$ glycerol tracer infusion in the post-absorptive phase revealed VLDL-TG concentrations to be reduced by $28 \%$, due to a $35 \%$ reduction in hepatic VLDL-TG secretion rate, whereas no differences in VLDL-TG clearance could be observed.

Thus, with exercise training, there is a decrease in hepatic VLDL-TG secretion. Although this indicates beneficial adaptation in liver metabolism, it cannot explain why exercise training lowers IHL content, since a lower VLDL-TG secretion would theoretically lead to more hepatic fat storage. Therefore, the reduced VLDL-TG secretion rate is most likely a consequence of the reduced IHL content upon exercise training. Concomitant with the lower VLDL-TG secretion after 6 months of exercise training in obese, T2DM patients, Alam et al. ${ }^{100}$ found a decrease in fasting 
FFA concentrations (from 0.9 to $0.5 \mathrm{mmol} / \mathrm{l}$ ) after exercise training. Thus, the lower FFA levels after exercise training may have contributed to a lower hepatic TG pool, which, in turn, may have resulted in a decrease in VLDL-TG secretion. However, in the sedentary non-obese young men fasting FFA concentrations were not altered after exercise training, although VLDL-TG secretion decreased with $35 \%{ }^{102}$. Therefore, physical activity training can alter VLDL-TG secretion also independently of FFA availability. Improved insulin sensitivity after exercise training may affect VLDL-apoB100 production directly. Insulin normally suppresses VLDL production and it has been shown in models of insulin resistance that VLDL export is deregulated with insulin resistance ${ }^{48}$. Currently, it is yet unknown how exercise training downregulates VLDL-TG metabolism mechanistically. More research is needed to directly link exercise training induced changes in VLDL metabolism to potential changes in IHL content.

\section{EFFECTS OF EXERCISE TRAINING ON HEPATIC MITOCHONDRIAL METABOLISM}

The effect of exercise training on hepatic mitochondrial function has not yet been investigated in humans. In rodents, several indices of hepatic mitochondrial content and function were assessed in OLETF rats after a voluntary wheel running ${ }^{96,98}$ or an exercise training program ${ }^{97}$. In these OLETF rats, exercise training reduced $^{96,97}$ or prevented ${ }^{98}$ hepatic fat storage concomitant with a decrease in key intermediates in hepatic fatty acid synthesis. Next to the effect on hepatic steatosis and DNL, these exercised OLETF rats increased complete palmitate oxidation to $\mathrm{CO}_{2}{ }^{96-98}$. Moreover, hepatic citrate synthase activity ${ }^{97,98}, \beta$-hydroxyacyl-CoA dehydrogenase activity ${ }^{97,98}$ and cytochrome $c$ protein content ${ }^{96}$ were significantly upregulated. High citrate synthase and $\beta$-hydroxyacyl-CoA dehydrogenase activity indicate increased aerobic capacity and mitochondrial density, whereas elevated cytochrome c protein content suggests enhanced final steps of oxidative phosphorylation. How exercise training might improve hepatic mitochondrial function is still unclear, but it might involve enhanced mitochondrial biogenesis. A study in mice showed that hepatic cytochrome $\mathrm{c}$ and cytochrome oxidase complex I protein content increased upon exercise training in wild-type mice ${ }^{103}$, but the increase was absent in PGC- $1 \alpha$ knock out mice ${ }^{103}$. This suggests PGC-1 $\alpha$ is required 
for the exercise training induced adaptations of mitochondrial oxidative proteins in mouse liver.

\section{EFFECTS OF ACUTE EXERCISE ON LIVER FAT METABOLISM}

An alternative way to explain the effects of exercise training on IHL content is by accumulating effects of one acute bout of exercise. However, the acute effects of exercise on liver fat metabolism have only been poorly investigated so far.

It is evident from measurements of substrate oxidation by indirect calorimetry that FA are a major source of fuel during low and moderate-intensity exercise on whole body level ${ }^{104}$. Stable isotope studies have revealed that only approximately $50 \%$ of total fat oxidation during exercise is accounted for by oxidation of plasma FFA ${ }^{105-}$ 107. This suggests that also other fat sources play a substantial role in energy provision ${ }^{108-110}$. Therefore, also hepatic TG may be exported from the liver to be oxidized. However, IHL content was reported to be either unchanged or increased after exercise ${ }^{104,111-113}$. Two studies reported a $35-40 \%$ increase in $\mathrm{IHL}$ content immediately after a $2 \mathrm{~h}$ exercise bout $\left(60 \% \mathrm{VO}_{2 \max }\right)$ in healthy, normal-weight participants ${ }^{111,112}$. In both studies, participants were put on an isocaloric, fat rich diet for either seventy-two ${ }^{111}$ or thirty-six ${ }^{112}$ hours prior to the exercise bout, a diet known to replete intramyocellular lipid stores ${ }^{111,112}$. After consuming a standardized meal, $\mathrm{IHL}$ content was measured before and immediately after $2 \mathrm{~h}$ of aerobic exercise. So, these 2 studies demonstrated that an acute exercise bout is not able to decrease IHL content after consuming a high-fat diet. Johnsen et al. ${ }^{104}$ then further examined whether acute exercise is able to decrease IHL content in healthy trained athletes, with and without consuming a high-fat diet. Trained participants were given an isocaloric, fat rich diet or an isocaloric control diet for $67 \mathrm{~h}$ prior to the acute exercise bout. IHL content was measured after an overnight fast, immediately after a $90 \mathrm{~min}$ exercise bout ( $65 \%$ of $\mathrm{VO}_{2 \text { peak }}$ ), and $4.5 \mathrm{~h}$ postexercise. After the $2^{\text {nd }} \mathrm{IHL}$ content quantification, a glucose beverage was given to minimize the risk of hypoglycemia. The authors found that $4.5 \mathrm{~h}$ post-exercise $\mathrm{IHL}$ content increased in both groups to a similar extent. It is known that performing exercise in the fasted state increases plasma FFA, which might have biased the 
observed exercise effects ${ }^{3,114}$. Therefore, we performed a study in men with overweight and NAFL ${ }^{113}$. Subjects performed $2 \mathrm{~h}$ of acute exercise ( $50 \%$ of $\mathrm{W}_{\max }$ ), once fasted (drinking water) and once glucose supplemented (drinking a glucose beverage) to suppress the plasma FFA levels. We found that $2 \mathrm{~h}$ of exercise in the fasted state increased IHL content significantly $4 \mathrm{~h}$ after exercise. IHL content was unchanged after exercise with glucose supplementation, however ${ }^{113}$.

Taken together, these data suggest acute exercise cannot lower IHL content and that the increase in plasma FFA induced by acute exercise might actually increase $\mathrm{IHL}$ content in the post-exercise phase. It has been shown that post-exercise splanchnic FFA uptake increases by $25 \%$ on average compared to pre-exercise in T2DM subjects ${ }^{115}$, and using stable isotope techniques it was calculated that approximately $50 \%$ of whole body re-esterification immediately after physical exercise ( $1 \mathrm{~h}$ at $60 \%$ of $\mathrm{VO}_{2 \text { peak }}$ ) occurs in the splanchnic area ${ }^{116}$. This data indicates that splanchnic (i.e. hepatic) FFA re-esterification might be an important factor immediately after exercise. So, while long-term exercise training results in lower FFA levels and decreased hepatic fat content, FFA are increased in the acute stimulation and hepatic TG content tends to increase after acute exercise in the fasted state.

\section{EFFECTS OF ACUTE EXERCISE ON LIPIDS ORIGINATING FROM A MEAL}

Acute exercise attenuates postprandial hypertriglyceridemia, which is generally described as a short-term effect ${ }^{117}$, and both pre- ${ }^{118-121}$ and post-meal ${ }^{122,123}$ exercise have shown to lower the TG response beneficially. This can be due to either altered TG clearance or decreased hepatic TG secretion, or a combination of the two.

There is evidence that changes in the magnitude of postprandial hypertriglyceridemia with pre-meal exercise are the result of an increase in muscle LPL activity ${ }^{117}$. For example plasma LPL, reflecting muscle LPL availability, increased by $20 \%$ the day after a pre-meal exercise bout in healthy young men ${ }^{124}$. After an acute exercise bout, LPL activity does not seem to increase immediately, but rather 4-8h after exercise ${ }^{125-127}$, and it is not maintained $20 \mathrm{~h}$ after the exercise bout ${ }^{126}$. Higher LPL activity has also been described in endurance-trained athletes ${ }^{88,89}$ and 
exercise training for 20 weeks increased post-heparin LPL activity in healthy, sedentary subjects ${ }^{90}$. Thus, the accumulated effect of acute exercise bouts on LPL activity might contribute to the observed effects in exercise training. Because of the narrow timeframe in which increased LPL activity is present after an acute bout of exercise, the increase in muscle LPL activity cannot completely explain the decrease in postprandial TG. Therefore, when exercise is performed immediately before a meal or after a meal, other mechanisms must exist, such as a reduction in hepatic VLDL-TG secretion ${ }^{128}$. Contrary to exercise training, acute exercise does not seem to affect $\mathrm{HL}$ activity ${ }^{118,123,124,129}$.

\section{EFFECTS OF ACUTE EXERCISE ON DE NOVO LIPOGENESIS}

De novo lipogenesis is increased by higher plasma glucose and higher insulin concentrations. Exercise training has been shown to lower glucose ${ }^{62,66}$ and insulin ${ }^{61,64,65,68,95}$ concentrations concomitant with decreases in IHL content, but the effect of exercise training on hepatic lipogenesis has not been investigated in humans. In contrast to exercise training, the effect of acute exercise on DNL has been tested in humans. Rabøl and colleagues ${ }^{130}$ investigated the effect of a single exercise bout on DNL directly. After 45 min of exercise or rest, followed by $\mathrm{IHL}$ content determination, healthy, young, lean but insulin resistant individuals were supplied with a high-carbohydrate liquid meal ( 55 energy \% carbohydrates), and deuterium-labeled water was given orally to measure DNL rates. It was shown that acute exercise significantly decreased DNL activity and this prevented the increase in IHL content that occurred in the postprandial condition, indicating that exercise can positively influence the rate of DNL after a subsequent meal. In this study, plasma glucose and insulin levels were not different between the exercise condition and the resting condition, but acute exercise induced a threefold increase in postprandial muscle glycogen synthesis. Thus, the single exercise bout redirected plasma glucose towards storage in skeletal muscle instead of liver, most likely via non-insulin-stimulated skeletal muscle glucose uptake by translocation of glucose transporter 4 (GLUT-4) $)^{131,132}$. Haus et al. ${ }^{133}$ reported that 7 consecutive days of exercise decreased the degree of saturation of IHL content when measured on the day after the last exercise bout. Since DNL results in saturated FA, changes in the degree of FA saturation in the liver may be indicative of changed DNL activity. In 
this study, fasting plasma insulin concentrations and the glucose incremental area under the curve during an OGTT were significantly improved after the last exercise bout. From these studies, it might be derived that the accumulated effect of single exercise bouts on lowering de novo lipogenesis by redirecting plasma glucose towards skeletal muscle, might contribute to the exercise training induced decrease in IHL content.

\section{EFFECTS OF ACUTE EXERCISE ON HEPATIC VLDL METABOLISM}

Exercise training reduces VLDL-TG secretion possibly due to a reduction in hepatic fat availability, although it cannot be excluded that exercise training may downregulate VLDL-TG metabolism directly. With acute exercise, plasma FFA increase and this may alter hepatic VLDL-TG secretion. Furthermore, changed hepatic VLDL-TG secretion and/or clearance may be important for the observed decrease in plasma TG after acute exercise. Two studies using VLDL-TG and palmitate tracers measured VLDL-secretion directly during and after 90min of aerobic exercise at $50 \%$ of the $\mathrm{VO}_{2 \max }{ }^{134,135}$. They found that in both healthy lean subjects and in overweight untrained men hepatic VLDL-TG secretion and clearance were reduced during exercise and in the early recovery phase. Thus, the increase in plasma FFA levels upon acute exercise does not seem to stimulate hepatic VLDL-TG secretion during exercise or in the early recovery phase. This misalignment might contribute to the observed increase in IHL content immediately ${ }^{111,112}$ and $4-5 \mathrm{~h}$ after exercise $^{104,113}$. Furthermore, because VLDL-TG secretion and clearance were both reduced to a similar extent, plasma VLDL-TG concentrations were not changed. Therefore it can be suggested that, in the early recovery phase, changes in VLDL-TG secretion and/or clearance do not contribute to decreases in plasma TG levels.

Plasma TG remain relatively low until the day after an acute exercise bout ${ }^{124}$, after which they return to baseline values. Although changes in VLDL-TG metabolism do not contribute to the decrease in plasma TG in the early recovery phase, it might be that they contribute to the sustained lower plasma TG the day after a single exercise bout ${ }^{124}$. It was found that an exercise bout of $1 \mathrm{~h}$ in the evening $(60 \%$ of $\mathrm{VO}_{2 \text { peak }}$ ) had no effect on VLDL-TG secretion and clearance on the following day in both men and women ${ }^{136,137}$. It has been shown that moderate-intensity exercise 
bouts of at least $2 \mathrm{~h}$ duration reduce plasma TG concentrations by approximately 30 $\%^{119,138-141}$, whereas shorter bouts of similar exercise had no effect on plasma TG concentrations ${ }^{142,143}$. Consistently, it was found that $2 \mathrm{~h}$ of evening exercise $(60 \%$ of the $\mathrm{VO}_{2 \text { peak }}$ ) did increase fasted VLDL-TG clearance rate by approximately $40 \%$ on the following day, without affecting VLDL-TG secretion in healthy, lean, young men $^{124}$. In women, a comparable exercise protocol decreased VLDL-TG secretion rate on the following day by approximately $22 \%$, concomitant with increased VLDLTG clearance ${ }^{144}$. Thus, performing acute exercise for $2 \mathrm{~h}$ in the evening induces changes in VLDL-TG metabolism on the following day that positively affect TG concentrations. However, the main effect is increased clearance of VLDL-TG from the plasma and only marginally involves changes in hepatic VLDL-TG secretion.

\section{EFFECTS OF ACUTE EXERCISE ON HEPATIC MITOCHONDRIAL METABOLISM}

As with exercise training, the effect of acute exercise on hepatic mitochondrial function has not been investigated in humans. In rodents, exercise training increases hepatic mitochondrial capacity and function, most likely via increased mitochondrial biogenesis. A single bout of acute exercise does not seem to increase hepatic mitochondrial protein capacity yet ${ }^{145}$. Also maximal hepatic mitochondrial oxidation, measured as cytochrome c oxidase activity, does not seem to increase immediately ${ }^{145,146}$. Interestingly, however, Haase et al. ${ }^{103}$ investigated hepatic cytochrome $\mathrm{c}$ and cytochrome oxidase complex I mRNA and protein content in PGC1- $\alpha$ knock-out mice and wild-type mice after acute exercise and exercise training. They found that, in wild-type mice, acute exercise increased hepatic cytochrome $\mathrm{c}$ and cytochrome oxidase complex I mRNA content, while exercise training increased hepatic cytochrome $\mathrm{c}$ and cytochrome oxidase complex I protein content. Interestingly, both effects were absent in PGC-1 $\alpha$ knock-out mice. This could suggest that, at least in rodents, PGC-1 $\alpha$ mediated effects after acute exercise might be responsible for the PGC-1 $\alpha$ mediated effects after exercise training. Further research in animal models and extrapolation to humans is needed to investigate if and how improved mitochondrial capacity contributes to changes in IHL content. 


\section{CONCLUSION}

NAFL develops due to higher hepatic fat availability and synthesis, which is not fully compensated by increased secretion and oxidation of hepatic TG. Exercise training lowers IHL content most likely via a reduction in hepatic fat availability and synthesis, and an increase in hepatic TG oxidation. A single bout of exercise seems to increase rather than decrease IHL content, and elevated plasma FFA during exercise and in the post-exercise period might be the main determinant. However, acute exercise might be responsible for several mediated effects observed after exercise training. Clearly, further research needs to be performed to better understand the underlying pathways and systems involved in the benefit of physical activity on hepatic metabolism and cross-talk with whole body metabolism. 


\section{REFERENCES}

1. Nguyen, T. \& Lau, D.C. The obesity epidemic and its impact on hypertension. Can J Cardiol 28, 326-333 (2012).

2. Ng, M., et al. Global, regional, and national prevalence of overweight and obesity in children and adults during 1980-2013: a systematic analysis for the Global Burden of Disease Study 2013. Lancet 384, 766-781 (2014).

3. Bilet, L., et al. Exercise-induced modulation of cardiac lipid content in healthy lean young men. Basic Res Cardiol 106, 307-315 (2011).

4. Lavoie, J.M. \& Gauthier, M.S. Regulation of fat metabolism in the liver: link to non-alcoholic hepatic steatosis and impact of physical exercise. Cell Mol Life Sci 63, 1393-1409 (2006).

5. Johnson, N.A., et al. Noninvasive assessment of hepatic lipid composition: Advancing understanding and management of fatty liver disorders. Hepatology 47, 1513-1523 (2008).

6. Day, C.P. Pathogenesis of steatohepatitis. Best Pract Res Clin Gastroenterol 16, 663-678 (2002).

7. Fabbrini, E., et al. Intrahepatic fat, not visceral fat, is linked with metabolic complications of obesity. Proc Natl Acad Sci U S A 106, 15430-15435 (2009).

8. Deivanayagam, S., et al. Nonalcoholic fatty liver disease is associated with hepatic and skeletal muscle insulin resistance in overweight adolescents. Am J Clin Nutr 88, 257-262 (2008).

9. Hwang, J.H., et al. Increased intrahepatic triglyceride is associated with peripheral insulin resistance: in vivo MR imaging and spectroscopy studies. Am J Physiol Endocrinol Metab 293, E1663-1669 (2007).

10. Reeder, S.B., Cruite, I., Hamilton, G. \& Sirlin, C.B. Quantitative assessment of liver fat with magnetic resonance imaging and spectroscopy. J Magn Reson Imaging 34, 729-749 (2011).

11. Bradbury, M.W. Lipid metabolism and liver inflammation. I. Hepatic fatty acid uptake: possible role in steatosis. American journal of physiology. Gastrointestinal and liver physiology 290, G194-198 (2006).

12. Tamura, S. \& Shimomura, I. Contribution of adipose tissue and de novo lipogenesis to nonalcoholic fatty liver disease. The Journal of clinical investigation 115, 1139-1142 (2005). 
13. Donnelly, K.L., et al. Sources of fatty acids stored in liver and secreted via lipoproteins in patients with nonalcoholic fatty liver disease. J Clin Invest 115, 1343-1351 (2005).

14. Iozzo, P., et al. Fatty acid metabolism in the liver, measured by positron emission tomography, is increased in obese individuals. Gastroenterology 139, 846-856, 856 e841-846 (2010).

15. Sunny, N.E., Parks, E.J., Browning, J.D. \& Burgess, S.C. Excessive hepatic mitochondrial TCA cycle and gluconeogenesis in humans with nonalcoholic fatty liver disease. Cell Metab 14, 804-810 (2011).

16. Nielsen, S., Guo, Z., Johnson, C.M., Hensrud, D.D. \& Jensen, M.D. Splanchnic lipolysis in human obesity. The Journal of clinical investigation 113, 15821588 (2004).

17. Holt, H.B., et al. Non-esterified fatty acid concentrations are independently associated with hepatic steatosis in obese subjects. Diabetologia 49, 141148 (2006).

18. Seppala-Lindroos, A., et al. Fat accumulation in the liver is associated with defects in insulin suppression of glucose production and serum free fatty acids independent of obesity in normal men. J Clin Endocrinol Metab 87, 3023-3028 (2002).

19. Tiikkainen, M., et al. Liver-fat accumulation and insulin resistance in obese women with previous gestational diabetes. Obes Res 10, 859-867 (2002).

20. van Hees, A.M., et al. Skeletal muscle fatty acid handling in insulin resistant men. Obesity 19, 1350-1359 (2011).

21. Most, J., Goossens, G.H., Jocken, J.W. \& Blaak, E.E. Short-term supplementation with a specific combination of dietary polyphenols increases energy expenditure and alters substrate metabolism in overweight subjects. International journal of obesity 38, 698-706 (2014).

22. Hsieh, J., Hayashi, A.A., Webb, J. \& Adeli, K. Postprandial dyslipidemia in insulin resistance: mechanisms and role of intestinal insulin sensitivity. Atheroscler Supp/ 9, 7-13 (2008).

23. Sanyal, A.J., et al. Nonalcoholic steatohepatitis: association of insulin resistance and mitochondrial abnormalities. Gastroenterology 120, 11831192 (2001). 
24. Armstrong, M.J., et al. Abdominal subcutaneous adipose tissue insulin resistance and lipolysis in patients with non-alcoholic steatohepatitis. Diabetes Obes Metab 16, 651-660 (2014).

25. Hussain, M.M. Intestinal lipid absorption and lipoprotein formation. Current opinion in lipidology 25, 200-206 (2014).

26. Berk, E.S., et al. Higher post-absorptive skeletal muscle LPL activity in African American vs. non-Hispanic White pre-menopausal women. Obesity 16, 199-201 (2008).

27. Ruge, T., Svensson, M., Eriksson, J.W. \& Olivecrona, G. Tissue-specific regulation of lipoprotein lipase in humans: effects of fasting. Eur J Clin Invest 35, 194-200 (2005).

28. Kersten, S. Physiological regulation of lipoprotein lipase. Biochimica et biophysica acta 1841, 919-933 (2014).

29. Jacome-Sosa, M.M. \& Parks, E.J. Fatty acid sources and their fluxes as they contribute to plasma triglyceride concentrations and fatty liver in humans. Current opinion in lipidology 25, 213-220 (2014).

30. Ryysy, L., et al. Hepatic fat content and insulin action on free fatty acids and glucose metabolism rather than insulin absorption are associated with insulin requirements during insulin therapy in type 2 diabetic patients. Diabetes 49, 749-758 (2000).

31. Barrows, B.R., Timlin, M.T. \& Parks, E.J. Spillover of dietary fatty acids and use of serum nonesterified fatty acids for the synthesis of VLDLtriacylglycerol under two different feeding regimens. Diabetes 54, 26682673 (2005).

32. Fraser, R., Dobbs, B.R. \& Rogers, G.W. Lipoproteins and the liver sieve: the role of the fenestrated sinusoidal endothelium in lipoprotein metabolism, atherosclerosis, and cirrhosis. Hepatology 21, 863-874 (1995).

33. Lindeboom, L., et al. Proton magnetic resonance spectroscopy reveals increased hepatic lipid content after a single high-fat meal with no additional modulation by added protein. Am J Clin Nutr 101, 65-71 (2015).

34. Utzschneider, K.M., et al. Beneficial effect of a weight-stable, low-fat/lowsaturated fat/low-glycaemic index diet to reduce liver fat in older subjects. The British journal of nutrition 109, 1096-1104 (2013).

35. van Herpen, N.A., Schrauwen-Hinderling, V.B., Schaart, G., Mensink, R.P. \& Schrauwen, $\mathrm{P}$. Three weeks on a high-fat diet increases intrahepatic lipid 
accumulation and decreases metabolic flexibility in healthy overweight men. J Clin Endocrinol Metab 96, E691-695 (2011).

36. Westerbacka, J., et al. Dietary fat content modifies liver fat in overweight nondiabetic subjects. The Journal of clinical endocrinology and metabolism 90, 2804-2809 (2005).

37. Green, C.J. \& Hodson, L. The influence of dietary fat on liver fat accumulation. Nutrients 6, 5018-5033 (2014).

38. Ameer, F., Scandiuzzi, L., Hasnain, S., Kalbacher, H. \& Zaidi, N. De novo lipogenesis in health and disease. Metabolism: clinical and experimental 63, 895-902 (2014).

39. Najjar, S.M., et al. Insulin acutely decreases hepatic fatty acid synthase activity. Cell metabolism 2, 43-53 (2005).

40. Griffiths, M.A., et al. Exercise down-regulates hepatic lipogenic enzymes in food-deprived and refed rats. J Nutr 126, 1959-1971 (1996).

41. Schwarz, J.M., Linfoot, P., Dare, D. \& Aghajanian, K. Hepatic de novo lipogenesis in normoinsulinemic and hyperinsulinemic subjects consuming high-fat, low-carbohydrate and low-fat, high-carbohydrate isoenergetic diets. Am J Clin Nutr 77, 43-50 (2003).

42. Lambert, J.E., Ramos-Roman, M.A., Browning, J.D. \& Parks, E.J. Increased de novo lipogenesis is a distinct characteristic of individuals with nonalcoholic fatty liver disease. Gastroenterology 146, 726-735 (2014).

43. Koteish, A. \& Diehl, A.M. Animal models of steatosis. Seminars in liver disease 21, 89-104 (2001).

44. Cano, A., et al. Hepatic VLDL assembly is disturbed in a rat model of nonalcoholic fatty liver disease: is there a role for dietary coenzyme Q? Journal of applied physiology 107, 707-717 (2009).

45. Visser, M.E., et al. Hepatic steatosis does not cause insulin resistance in people with familial hypobetalipoproteinaemia. Diabetologia 54, 21132121 (2011).

46. Fabbrini, E., et al. Alterations in adipose tissue and hepatic lipid kinetics in obese men and women with nonalcoholic fatty liver disease. Gastroenterology 134, 424-431 (2008).

47. Adiels, M., et al. Overproduction of large VLDL particles is driven by increased liver fat content in man. Diabetologia 49, 755-765 (2006). 
48. Annuzzi, G., et al. Insulin resistance is independently associated with postprandial alterations of triglyceride-rich lipoproteins in type 2 diabetes mellitus. Arterioscler Thromb Vasc Biol 24, 2397-2402 (2004).

49. Cassader, M., et al. Postprandial triglyceride-rich lipoprotein metabolism and insulin sensitivity in nonalcoholic steatohepatitis patients. Lipids 36, 1117-1124 (2001).

50. Magkos, F., Fabbrini, E., Mohammed, B.S., Patterson, B.W. \& Klein, S. Increased whole-body adiposity without a concomitant increase in liver fat is not associated with augmented metabolic dysfunction. Obesity (Silver Spring) 18, 1510-1515 (2010).

51. McGarry, J.D. \& Foster, D.W. Regulation of hepatic fatty acid oxidation and ketone body production. Annu Rev Biochem 49, 395-420 (1980).

52. Schofield, P.S., Sugden, M.C., Corstorphine, C.G. \& Zammit, V.A. Altered interactions between lipogenesis and fatty acid oxidation in regenerating rat liver. Biochem J 241, 469-474 (1987).

53. Koliaki, C., et al. Adaptation of hepatic mitochondrial function in humans with non-alcoholic Fatty liver is lost in steatohepatitis. Cell metabolism 21, 739-746 (2015).

54. Cortez-Pinto, H., et al. Alterations in liver ATP homeostasis in human nonalcoholic steatohepatitis: a pilot study. JAMA 282, 1659-1664 (1999).

55. Otsuka, H., Harada, M., Koga, K. \& Nishitani, H. Effects of hepatic impairment on the metabolism of fructose and 5-fluorouracil, as studied in fatty liver models using in vivo 31P-MRS and 19F-MRS. Magn Reson Imaging 17, 283-290 (1999).

56. Perez-Carreras, M., et al. Defective hepatic mitochondrial respiratory chain in patients with nonalcoholic steatohepatitis. Hepatology 38, 999-1007 (2003).

57. Kantartzis, K., et al. High cardiorespiratory fitness is an independent predictor of the reduction in liver fat during a lifestyle intervention in nonalcoholic fatty liver disease. Gut 58, 1281-1288 (2009).

58. Church, T.S., et al. Association of cardiorespiratory fitness, body mass index, and waist circumference to nonalcoholic fatty liver disease. Gastroenterology 130, 2023-2030 (2006).

59. Perseghin, G., et al. Habitual physical activity is associated with intrahepatic fat content in humans. Diabetes Care 30, 683-688 (2007). 
60. Bacchi, E., et al. Both resistance training and aerobic training reduce hepatic fat content in type 2 diabetic subjects with NAFLD (The RAED2 randomized trial). Hepatology (2013).

61. Finucane, F.M., et al. The effects of aerobic exercise on metabolic risk, insulin sensitivity and intrahepatic lipid in healthy older people from the Hertfordshire Cohort Study: a randomised controlled trial. Diabetologia 53, 624-631 (2010).

62. Hallsworth, K., et al. Resistance exercise reduces liver fat and its mediators in non-alcoholic fatty liver disease independent of weight loss. Gut 60, 1278-1283 (2011).

63. Johnson, N.A., et al. Aerobic exercise training reduces hepatic and visceral lipids in obese individuals without weight loss. Hepatology 50, 1105-1112 (2009).

64. Lee, S., et al. Effects of aerobic versus resistance exercise without caloric restriction on abdominal fat, intrahepatic lipid, and insulin sensitivity in obese adolescent boys: a randomized, controlled trial. Diabetes 61, 27872795 (2012).

65. Lee, S., et al. Aerobic exercise but not resistance exercise reduces intrahepatic lipid content and visceral fat and improves insulin sensitivity in obese adolescent girls: a randomized controlled trial. Am J Physiol Endocrinol Metab 305, E1222-1229 (2013).

66. Pugh, C.J., et al. Exercise training reverses endothelial dysfunction in nonalcoholic fatty liver disease. Am J Physiol Heart Circ Physiol (2014).

67. Sullivan, S., Kirk, E.P., Mittendorfer, B., Patterson, B.W. \& Klein, S. Randomized trial of exercise effect on intrahepatic triglyceride content and lipid kinetics in nonalcoholic fatty liver disease. Hepatology 55, 1738-1745 (2012).

68. van der Heijden, G.J., et al. A 12-week aerobic exercise program reduces hepatic fat accumulation and insulin resistance in obese, Hispanic adolescents. Obesity (Silver Spring) 18, 384-390 (2010).

69. Palmer, M. \& Schaffner, F. Effect of weight reduction on hepatic abnormalities in overweight patients. Gastroenterology 99, 1408-1413 (1990).

70. Phielix, E., et al. High oxidative capacity due to chronic exercise training attenuates lipid-induced insulin resistance. Diabetes 61, 2472-2478 (2012). 
71. You, T., Berman, D.M., Ryan, A.S. \& Nicklas, B.J. Effects of hypocaloric diet and exercise training on inflammation and adipocyte lipolysis in obese postmenopausal women. The Journal of clinical endocrinology and metabolism 89, 1739-1746 (2004).

72. Shojaee-Moradie, F., et al. Exercise training reduces fatty acid availability and improves the insulin sensitivity of glucose metabolism. Diabetologia 50, 404-413 (2007).

73. Lange, K.H., et al. Endurance training and GH administration in elderly women: effects on abdominal adipose tissue lipolysis. American journal of physiology. Endocrinology and metabolism 280, E886-897 (2001).

74. Hickner, R.C., Racette, S.B., Binder, E.F., Fisher, J.S. \& Kohrt, W.M. Suppression of whole body and regional lipolysis by insulin: effects of obesity and exercise. The Journal of clinical endocrinology and metabolism 84, 3886-3895 (1999).

75. Meex, R.C., et al. Restoration of muscle mitochondrial function and metabolic flexibility in type 2 diabetes by exercise training is paralleled by increased myocellular fat storage and improved insulin sensitivity. Diabetes 59, 572-579 (2010).

76. Polak, J., et al. Dynamic strength training improves insulin sensitivity and functional balance between adrenergic alpha $2 \mathrm{~A}$ and beta pathways in subcutaneous adipose tissue of obese subjects. Diabetologia 48, 26312640 (2005).

77. Malin, S.K., et al. Insulin sensitivity and metabolic flexibility following exercise training among different obese insulin-resistant phenotypes. American journal of physiology. Endocrinology and metabolism 305, E12921298 (2013).

78. Solomon, T.P., Haus, J.M., Marchetti, C.M., Stanley, W.C. \& Kirwan, J.P. Effects of exercise training and diet on lipid kinetics during free fatty acidinduced insulin resistance in older obese humans with impaired glucose tolerance. American journal of physiology. Endocrinology and metabolism 297, E552-559 (2009).

79. DiPietro, L., Dziura, J., Yeckel, C.W. \& Neufer, P.D. Exercise and improved insulin sensitivity in older women: evidence of the enduring benefits of higher intensity training. Journal of applied physiology 100, 142-149 (2006). 
80. de Glisezinski, I., et al. Aerobic training improves exercise-induced lipolysis in SCAT and lipid utilization in overweight men. American journal of physiology. Endocrinology and metabolism 285, E984-990 (2003).

81. Tunstall, R.J., et al. Exercise training increases lipid metabolism gene expression in human skeletal muscle. American journal of physiology. Endocrinology and metabolism 283, E66-72 (2002).

82. Iozzo, P., et al. Effect of training status on regional disposal of circulating free fatty acids in the liver and skeletal muscle during physiological hyperinsulinemia. Diabetes Care 27, 2172-2177 (2004).

83. Hannukainen, J.C., et al. Increased physical activity decreases hepatic free fatty acid uptake: a study in human monozygotic twins. The Journal of physiology 578, 347-358 (2007).

84. Patsch, J.R., Karlin, J.B., Scott, L.W., Smith, L.C. \& Gotto, A.M., Jr. Inverse relationship between blood levels of high density lipoprotein subfraction 2 and magnitude of postprandial lipemia. Proceedings of the National Academy of Sciences of the United States of America 80, 1449-1453 (1983).

85. Ziogas, G.G., Thomas, T.R. \& Harris, W.S. Exercise training, postprandial hypertriglyceridemia, and LDL subfraction distribution. Med Sci Sports Exerc 29, 986-991 (1997).

86. Cohen, J.C., Noakes, T.D. \& Benade, A.J. Postprandial lipemia and chylomicron clearance in athletes and in sedentary men. The American journal of clinical nutrition 49, 443-447 (1989).

87. Neuger, L., et al. Effects of heparin on the uptake of lipoprotein lipase in rat liver. BMC Physiol 4, 13 (2004).

88. Kantor, M.A., Cullinane, E.M., Sady, S.P., Herbert, P.N. \& Thompson, P.D. Exercise acutely increases high density lipoprotein-cholesterol and lipoprotein lipase activity in trained and untrained men. Metabolism: clinical and experimental 36, 188-192 (1987).

89. Podl, T.R., et al. Lipoprotein lipase activity and plasma triglyceride clearance are elevated in endurance-trained women. Metabolism: clinical and experimental 43, 808-813 (1994).

90. Ukkola, O., et al. Genetic variation at the lipoprotein lipase locus and plasma lipoprotein and insulin levels in the Quebec Family Study. Atherosclerosis 158, 199-206 (2001). 
91. Brunzell, J.D., et al. Heterogeneity of primary lipoprotein lipase deficiency. Metabolism: clinical and experimental 29, 624-629 (1980).

92. Teran-Garcia, M., et al. Hepatic lipase gene variant $-514 C>T$ is associated with lipoprotein and insulin sensitivity response to regular exercise: the HERITAGE Family Study. Diabetes 54, 2251-2255 (2005).

93. Jansen, $\mathrm{H}$. Hepatic lipase: friend or foe and under what circumstances? Curr Atheroscler Rep 6, 343-347 (2004).

94. van Loon, L.J., et al. Intramyocellular lipid content in type 2 diabetes patients compared with overweight sedentary men and highly trained endurance athletes. American journal of physiology. Endocrinology and metabolism 287, E558-565 (2004).

95. St George, A., et al. Independent effects of physical activity in patients with nonalcoholic fatty liver disease. Hepatology 50, 68-76 (2009).

96. Rector, R.S., et al. Daily exercise increases hepatic fatty acid oxidation and prevents steatosis in Otsuka Long-Evans Tokushima Fatty rats. American journal of physiology. Gastrointestinal and liver physiology 294, G619-626 (2008).

97. Linden, M.A., et al. Combining metformin and aerobic exercise training in the treatment of type 2 diabetes and NAFLD in OLETF rats. American journal of physiology. Endocrinology and metabolism 306, E300-310 (2014).

98. Rector, R.S., et al. Daily exercise vs. caloric restriction for prevention of nonalcoholic fatty liver disease in the OLETF rat model. American journal of physiology. Gastrointestinal and liver physiology 300, G874-883 (2011).

99. Linden, M.A., et al. Hepatic steatosis development with four weeks of physical inactivity in previously active, hyperphagic OLETF rats. Am J Physiol Regul Integr Comp Physiol 304, R763-771 (2013).

100. Alam, S., et al. The effect of a six-month exercise program on very lowdensity lipoprotein apolipoprotein B secretion in type 2 diabetes. J Clin Endocrinol Metab 89, 688-694 (2004).

101. Kissebah, A.H., Alfarsi, S., Evans, D.J. \& Adams, P.W. Integrated regulation of very low density lipoprotein triglyceride and apolipoprotein-B kinetics in non-insulin-dependent diabetes mellitus. Diabetes 31, 217-225 (1982).

102. Tsekouras, Y.E., et al. High-intensity interval aerobic training reduces hepatic very low-density lipoprotein-triglyceride secretion rate in men. $A m$ J Physiol Endocrinol Metab 295, E851-858 (2008). 
103. Haase, T.N., et al. Role of PGC-1alpha in exercise and fasting-induced adaptations in mouse liver. Am J Physiol Regul Integr Comp Physiol 301, R1501-1509 (2011).

104. Johnson, N.A., et al. Effect of prolonged exercise and pre-exercise dietary manipulation on hepatic triglycerides in trained men. Eur J Appl Physiol 112, 1817-1825 (2012).

105. Romijn, J.A., et al. Regulation of endogenous fat and carbohydrate metabolism in relation to exercise intensity and duration. Am J Physiol 265, E380-391 (1993).

106. van Loon, L.J., Greenhaff, P.L., Constantin-Teodosiu, D., Saris, W.H. \& Wagenmakers, A.J. The effects of increasing exercise intensity on muscle fuel utilisation in humans. The Journal of physiology 536, 295-304 (2001).

107. Martin, W.H., 3rd, et al. Effect of endurance training on plasma free fatty acid turnover and oxidation during exercise. The American journal of physiology 265, E708-714 (1993).

108. Tamura, Y., et al. Effects of diet and exercise on muscle and liver intracellular lipid contents and insulin sensitivity in type 2 diabetic patients. J Clin Endocrinol Metab 90, 3191-3196 (2005).

109. Lewis, G.F., Carpentier, A., Adeli, K. \& Giacca, A. Disordered fat storage and mobilization in the pathogenesis of insulin resistance and type 2 diabetes. Endocr Rev 23, 201-229 (2002).

110. Wei, Y., Rector, R.S., Thyfault, J.P. \& Ibdah, J.A. Nonalcoholic fatty liver disease and mitochondrial dysfunction. World J Gastroenterol 14, 193-199 (2008).

111. Bucher, J., et al. The effect of a single $2 \mathrm{~h}$ bout of aerobic exercise on ectopic lipids in skeletal muscle, liver and the myocardium. Diabetologia (2014).

112. Egger, A., et al. The effect of aerobic exercise on intrahepatocellular and intramyocellular lipids in healthy subjects. PLoS One 8, e70865 (2013).

113. Bilet, L., et al. Acute exercise does not decrease liver fat in men with overweight or NAFLD. Sci Rep 5, 9709 (2015).

114. Schrauwen, P., et al. Effect of acute exercise on uncoupling protein 3 is a fat metabolism-mediated effect. American journal of physiology. Endocrinology and metabolism 282, E11-17 (2002). 
115. Simonsen, L., Henriksen, O., Enevoldsen, L.H. \& Bulow, J. The effect of exercise on regional adipose tissue and splanchnic lipid metabolism in overweight type 2 diabetic subjects. Diabetologia 47, 652-659 (2004).

116. Van Hall, G., et al. Regional fat metabolism in human splanchnic and adipose tissues; the effect of exercise. The Journal of physiology 543, 10331046 (2002).

117. Katsanos, C.S. Prescribing aerobic exercise for the regulation of postprandial lipid metabolism : current research and recommendations. Sports medicine 36, 547-560 (2006).

118. Zhang, J.Q., Thomas, T.R. \& Ball, S.D. Effect of exercise timing on postprandial lipemia and HDL cholesterol subfractions. Journal of applied physiology 85, 1516-1522 (1998).

119. Tsetsonis, N.V. \& Hardman, A.E. Effects of low and moderate intensity treadmill walking on postprandial lipaemia in healthy young adults. Eur J Appl Physiol Occup Physiol 73, 419-426 (1996).

120. Tsetsonis, N.V. \& Hardman, A.E. Reduction in postprandial lipemia after walking: influence of exercise intensity. Med Sci Sports Exerc 28, 1235-1242 (1996).

121. Aldred, H.E., Perry, I.C. \& Hardman, A.E. The effect of a single bout of brisk walking on postprandial lipemia in normolipidemic young adults. Metabolism: clinical and experimental 43, 836-841 (1994).

122. Katsanos, C.S. \& Moffatt, R.J. Acute effects of premeal versus postmeal exercise on postprandial hypertriglyceridemia. Clin J Sport Med 14, 33-39 (2004).

123. Hardman, A.E. \& Aldred, H.E. Walking during the postprandial period decreases alimentary lipaemia. J Cardiovasc Risk 2, 71-78 (1995).

124. Magkos, F., Wright, D.C., Patterson, B.W., Mohammed, B.S. \& Mittendorfer, B. Lipid metabolism response to a single, prolonged bout of endurance exercise in healthy young men. Am J Physiol Endocrinol Metab 290, E355-362 (2006).

125. Seip, R.L. \& Semenkovich, C.F. Skeletal muscle lipoprotein lipase: molecular regulation and physiological effects in relation to exercise. Exerc Sport Sci Rev 26, 191-218 (1998). 
126. Seip, R.L., Mair, K., Cole, T.G. \& Semenkovich, C.F. Induction of human skeletal muscle lipoprotein lipase gene expression by short-term exercise is transient. The American journal of physiology 272, E255-261 (1997).

127. Kiens, B., Lithell, H., Mikines, K.J. \& Richter, E.A. Effects of insulin and exercise on muscle lipoprotein lipase activity in man and its relation to insulin action. The Journal of clinical investigation 84, 1124-1129 (1989).

128. Borsheim, E., Knardahl, S. \& Hostmark, A.T. Short-term effects of exercise on plasma very low density lipoproteins (VLDL) and fatty acids. Med Sci Sports Exerc 31, 522-530 (1999).

129. Katsanos, C.S., Grandjean, P.W. \& Moffatt, R.J. Effects of low and moderate exercise intensity on postprandial lipemia and postheparin plasma lipoprotein lipase activity in physically active men. Journal of applied physiology 96, 181-188 (2004).

130. Rabol, R., Petersen, K.F., Dufour, S., Flannery, C. \& Shulman, G.I. Reversal of muscle insulin resistance with exercise reduces postprandial hepatic de novo lipogenesis in insulin resistant individuals. Proc Natl Acad Sci U S A 108, 13705-13709 (2011).

131. Richter, E.A. \& Hargreaves, M. Exercise, GLUT4, and skeletal muscle glucose uptake. Physiol Rev 93, 993-1017 (2013).

132. Stanford, K.I. \& Goodyear, L.J. Exercise and type 2 diabetes: molecular mechanisms regulating glucose uptake in skeletal muscle. Adv Physiol Educ 38, 308-314 (2014).

133. Haus, J.M., et al. Improved hepatic lipid composition following short-term exercise in nonalcoholic Fatty liver disease. J Clin Endocrinol Metab 98, E1181-1188 (2013).

134. Sondergaard, E., et al. Effects of exercise on VLDL-triglyceride oxidation and turnover. American journal of physiology. Endocrinology and metabolism 300, E939-944 (2011).

135. Nellemann, B., et al. Kinetics and utilization of lipid sources during acute exercise and acipimox. American journal of physiology. Endocrinology and metabolism 307, E199-208 (2014).

136. Magkos, F., Patterson, B.W., Mohammed, B.S. \& Mittendorfer, B. Basal adipose tissue and hepatic lipid kinetics are not affected by a single exercise bout of moderate duration and intensity in sedentary women. Clin Sci (Lond) 116, 327-334 (2009). 
137. Magkos, F., Patterson, B.W., Mohammed, B.S. \& Mittendorfer, B. A single 1-h bout of evening exercise increases basal FFA flux without affecting VLDL-triglyceride and VLDL-apolipoprotein B-100 kinetics in untrained lean men. Am J Physiol Endocrinol Metab 292, E1568-1574 (2007).

138. Annuzzi, G., Jansson, E., Kaijser, L., Holmquist, L. \& Carlson, L.A. Increased removal rate of exogenous triglycerides after prolonged exercise in man: time course and effect of exercise duration. Metabolism: clinical and experimental 36, 438-443 (1987).

139. Cullinane, E., Siconolfi, S., Saritelli, A. \& Thompson, P.D. Acute decrease in serum triglycerides with exercise: is there a threshold for an exercise effect? Metabolism 31, 844-847 (1982).

140. Ferguson, M.A., et al. Effects of four different single exercise sessions on lipids, lipoproteins, and lipoprotein lipase. Journal of applied physiology 85 , 1169-1174 (1998).

141. Gill, J.M., et al. Effects of short-term detraining on postprandial metabolism, endothelial function, and inflammation in endurance-trained men: dissociation between changes in triglyceride metabolism and endothelial function. j.gill@bio.gla.ac.uk. The Journal of clinical endocrinology and metabolism 88, 4328-4335 (2003).

142. Cullinane, E., Lazarus, B., Thompson, P.D., Saratelli, A. \& Herbert, P.N. Acute effects of a single exercise session on serum lipids in untrained men. Clin Chim Acta 109, 341-344 (1981).

143. Altena, T.S., Michaelson, J.L., Ball, S.D. \& Thomas, T.R. Single sessions of intermittent and continuous exercise and postprandial lipemia. Med Sci Sports Exerc 36, 1364-1371 (2004).

144. Bellou, E., et al. Acute effects of exercise and calorie restriction on triglyceride metabolism in women. Med Sci Sports Exerc 45, 455-461 (2013).

145. Venditti, P., Masullo, P. \& Meo, S.D. Effect of exercise duration on characteristics of mitochondrial population from rat liver. Arch Biochem Biophys 368, 112-120 (1999).

146. Venditti, P., De Rosa, R., Caldarone, G. \& Di Meo, S. Effect of prolonged exercise on oxidative damage and susceptibility to oxidants of rat tissues in severe hyperthyroidism. Arch Biochem Biophys 442, 229-237 (2005). 




\section{CHAPTER 3}

\section{HEPATIC, SKELETAL MUSCLE AND ADIPOSE}

TISSUE INSULIN SENSITIVITY IS SIMILARLY IMPAIRED IN MEN WITH NAFL AND IN TYPE 2 DIABETIC PATIENTS

Bram Brouwers, Vera B. Schrauwen-Hinderling, Anne Gemmink, Bas Havekes, Yvonne Bruls, Dennis Dahlmans, Patrick Schrauwen, Matthijs K.C. Hesselink

An adjusted version has been submitted 


\section{ABSTRACT}

A strong association between non-alcoholic fatty liver (NAFL) and insulin resistance has been reported and people with increased intrahepatic lipid (IHL) content are characterized by reduced hepatic insulin sensitivity and compromised postprandial glucose clearance. Here, we examined the metabolic disturbances related to insulin sensitivity in obese patients with or without NAFL and compared the severity of these aberrations to BMI-matched patients with overt type 2 diabetes mellitus (T2DM).

Eleven sedentary overweight/obese male control (CTRL) subjects, 11 male NAFL subjects and 13 male T2DM patients of comparable body weight, BMI and aerobic capacity $\left(\mathrm{VO}_{2 \max }\right)$ participated in this study. IHL content (proton magnetic resonance spectroscopy $\left({ }^{1} \mathrm{H}-\mathrm{MRS}\right)$ ), insulin sensitivity (2-step hyperinsulinemic-euglycemic clamp) and substrate oxidation (indirect calorimetry) were measured.

T2DM patients had elevated fasting plasma glucose (FPG) levels, whereas NAFL and CTRL subjects were normoglycemic $(p<0.001)$. Fasting insulin levels in NAFL subjects were similar to T2DM patients, and significantly higher compared to CTRL subjects $(p=0.012)$. Insulin stimulation of glucose disposal (delta $R_{d}$ ) was comparable in NAFL subjects and T2DM patients, and this was significantly lower than in CTRL subjects $(p<0.001)$. Also suppression of endogenous glucose production $(E G P)(p<0.001)$ and suppression of plasma free fatty acids (FFA) $(p=0.030)$ was similarly impaired in NAFL subjects and T2DM patients $(p>0.05)$ when compared to CTRL subjects.

Thus, NAFL subjects are characterized by hepatic, skeletal muscle and adipose tissue insulin resistance, which is already reduced to a similar extent as in patients with overt T2DM. Therefore, in people with NAFL, early diagnosis and proper treatment of insulin resistance is necessary to prevent development of type 2 diabetes mellitus. 


\section{INTRODUCTION}

Type 2 diabetes mellitus (T2DM) originates from a sustained reduction in insulin sensitivity. Thus, in the T2DM state insulin-mediated glucose uptake is compromised and the inhibitory effect of insulin on adipose tissue lipolysis and hepatic glucose production is alleviated. As a consequence, postprandial glucose levels in the blood are elevated, as are levels of free fatty acids. Progression from the early stages of insulin resistance (when glycemic control is not yet abnormal) to overt T2DM is a gradual and underdiagnosed process. In this process the distinction is made between normal glucose tolerance (NGT), impaired glucose tolerance (IGT), impaired fasting glucose (IFG) and overt T2DM ${ }^{1}$. IGT is referred to as an abnormal response to an oral glucose tolerance test ( $2 \mathrm{~h}$ oral glucose tolerance test (OGTT) values between 7.8 and $11.0 \mathrm{mmol} / \mathrm{L})^{2}$. The diagnosis of IFG and overt T2DM includes elevated fasted glucose levels, most commonly attributed to elevated hepatic glucose output due to resistance of the liver to the inhibitory effect of insulin on hepatic glucose production.

Impaired hepatic insulin sensitivity has been hypothesized to originate from increased hepatic fat storage ${ }^{3}$. Hepatic fat (intrahepatic lipid, $\mathrm{IHL}$ ) content is subject to rapid fluctuations, even upon physiological stimuli like exercise and nutrition ${ }^{4,5}$. A sustained elevation of liver fat (steatosis), however, is associated with obesity ${ }^{6}$ and compromised hepatic insulin sensitivity ${ }^{6-12}$. If hepatic fat content exceeds 5.0 $\%$ of the total liver's weight, and is of non-alcoholic origin, this condition is referred to as non-alcoholic fatty liver (NAFL) ${ }^{13}$.

A strong association between NAFL and insulin resistance has been reported frequently,6-12 and subjects with increased IHL content were characterized by reduced hepatic insulin sensitivity and compromised postprandial glucose clearance compared to people with normal IHL content ${ }^{7,8,11}$. Moreover, fasting insulin levels were significantly higher in people with increased IHL content, and it has been shown that IHL content strongly correlates with fasting plasma insulin and fasting C-peptide concentrations ${ }^{14-16}$. Interestingly, prospective studies revealed that elevations in liver enzymes precede the development of $\mathrm{T}_{2} \mathrm{DM}^{17,18}$. In a 4 year prospective case-control study obese (BMI $28.7 \mathrm{~kg} / \mathrm{m}^{2}$ ) subjects diagnosed with NAFL (cases) were compared to lean (BMI $23.0 \mathrm{~kg} / \mathrm{m}^{2}$ ) subjects without NAFL 
(controls) ${ }^{19}$. The obese subjects with NAFL showed more progression of the disease over these 4 years with higher prevalence and more severe metabolic disturbances in the obese subjects with NAFL, relative to the lean without NAFL. Although these data indicate that a fatty liver promotes the vulnerability to progressive development of metabolic disturbances, it does not exclude that obesity per se is also a major contributing factor to the progressive metabolic aberrations observed in obese NAFL patients.

Therefore, we aimed to examine the metabolic disturbances related to insulin sensitivity in overweight/obese patients with or without NAFL. Moreover, we aimed to compare the severity of these aberrations to BMI-matched patients with overt T2DM. We hypothesized that the markers of insulin resistance in subjects with NAFL would be intermediate compared to BMI-matched people without NAFL and BMI-matched T2DM patients.

\section{RESEARCH DESIGN AND METHODS}

Eleven overweight/obese male non-NAFL control (CTRL) subjects, 11 male NAFL subjects and 13 male T2DM patients, matched for BMI, were included in the study. Subjects were sedentary, middle-aged ( $40-70$ years old) men with stable dietary habits. General exclusion criteria were unstable body weight, cardiac disease, impaired renal function, hemoglobin $<7.5 \mathrm{mmol} / \mathrm{l}$, use of beta-blockers, use of antithrombotic medication, elevated blood pressure (> 160/100 $\mathrm{mmHg}$ ), claustrophobia, contra-indications for MRI, recent participation in a weight loss or vigorous exercise program, history of substantial alcohol use ( $>3$ units per day), history of drug abuse, use of insulin therapy and use of medication known to interfere with glucose homeostasis (except for antidiabetic medication in T2DM patients). CTRL subjects needed to have a liver fat content $\leq 4 \%$ when measured with ${ }^{1} \mathrm{H}-\mathrm{MRS}$, in the absence of clinical signs of liver disease or liver dysfunction (ALAT > $2.5 \mathrm{x}$ increased), and had to be normoglycemic according to the WHO criteria. NAFL subjects needed to have a liver fat content $\geq 5 \%$ when measured with ${ }^{1} \mathrm{H}-\mathrm{MRS}$, with a fasting plasma glucose (FPG) concentration $<7.0 \mathrm{mmol} / \mathrm{l}$. T2DM patients were allowed to be on sulphonyl urea or metformin therapy for at least 6 months with constant dose for at least 2 months or on a dietary treatment 
for 6 months, with FPG concentration $\geq 7.0 \mathrm{mmol} / \mathrm{l}$ and $<10.0 \mathrm{mmol} / \mathrm{l}$ at the time of screening. T2DM patients were not screened for liver fat content.

Subjects were recruited via advertisements in local newspapers. Before the onset of the study, routine medical laboratory testing, a medical history and physical examination was performed, and a resting electrocardiogram was taken. Maximal work load $\left(\mathrm{W}_{\max }\right)$ and maximal aerobic capacity $\left(\mathrm{VO}_{2 \max }\right)$ were assessed during a graded cycling test with concurrent ECG until exhaustion ${ }^{20}$. Body composition was determined by DXA (Hologic Discovery A, Waltham, MA, USA).

All subjects gave written informed consent before participation in the study. The Maastricht University Medical Ethical Committee had approved the study. The study was performed according to the principles expressed in the most recent version of the declaration of Helsinki.

\section{PROTON MAGNETIC RESONANCE SPECTROSCOPY}

IHL content was measured in the morning (6.00 - 7.00 A.M.) of the 2-step hyperinsulinemic-euglycemic clamp test day. Proton magnetic resonance spectroscopy ( $\left.{ }^{1} \mathrm{H}-\mathrm{MRS}\right)$ was used to quantify IHL content as described earlier ${ }^{4}$, however using a five element coil on a $3 \mathrm{~T}$ whole body scanner (Philips Achieva 3Tx; Philips Healthcare, Best, The Netherlands) and a repetition time $=4000 \mathrm{msec}$, echo time $=32.5 \mathrm{msec}$, and number of averages $=64$. To minimize motion artifacts, subjects were asked to breathe in the rhythm of the measurement and to be at end-expiration during acquisition of spectra. To determine the intensity of the lipid peak, the water signal was suppressed using frequency-selective prepulses. The unsuppressed water resonance was used as internal reference (number of averages = 32) and spectra were fitted with MATLAB R2014b (Mathworks, Natick, MA, USA). Values are given as T2-corrected ratios of the $\mathrm{CH}_{2}$ resonance, relative to the unsuppressed water resonance, as percentage. 


\section{PHOSPHORUS MAGNETIC RESONANCE SPECTROSCOPY}

Measurements were performed on a $3 \mathrm{~T}$ whole body scanner (Philips Achieva 3Tx; Philips Healthcare, Best, The Netherlands). Subjects were positioned in a supine position. A $14 \mathrm{~cm}$ diameter circular ${ }^{31} \mathrm{P}$ receive and transmit surface coil was positioned at the level of the liver and secured in place using Velcro bands. Before spectroscopic acquisition, fifteen axial, coronal and sagittal ${ }^{1} \mathrm{H}-\mathrm{MR}$ images (slice thickness $=10 \mathrm{~mm}$, field of view $=450 * 450 \mathrm{~mm}$, matrix size $=192 * 96, \mathrm{TE}=1.82$ $\mathrm{msec}, \mathrm{TR}=3.8 \mathrm{msec}$ ) from the liver region were acquired using a gradient-echo sequence during one breath hold. The VOI was positioned parallel to the coil at a distance of $5 \mathrm{~cm}$ from the center of the coil. Manual tuning and matching of the ${ }^{31} \mathrm{P}$ receive and transmit surface coil was performed and pencil bean shimming was performed. Localized phosphorus spectra were obtained using Image Selected In Vivo Spectroscopy (ISIS). Sequence parameters were repetition time (TR): 5500 msec, number of single acquisitions (NSA): 128, number of sample points: 1024 and spectral bandwidth: $3000 \mathrm{~Hz}$. The carrier frequency was chosen to match the resonance frequency of $\mathrm{y}$-ATP.

\section{HYPERINSULINEMIC-EUGLYCEMIC CLAMP}

All participants underwent a 2-step $6 \mathrm{~h}$ hyperinsulinemic-euglycemic clamp (10 and $\left.40 \mathrm{mU} / \mathrm{m}^{2} / \mathrm{min}\right)^{21}$. After an overnight fast, participants received a primed continuous infusion of $\left[6,6-{ }^{2} \mathrm{H}_{2}\right]$ glucose $(0.04 \mathrm{mg} / \mathrm{kg} / \mathrm{min})$ to determine rates of endogenous glucose production (EGP) and whole body glucose disposal rates $\left(R_{d}\right)$ as previously described ${ }^{22}$. After $180 \mathrm{~min}$, low-dose insulin infusion was started (10 $\mathrm{mU} / \mathrm{m}^{2} / \mathrm{min}$ ) for $3.5 \mathrm{~h}$ until a steady state was reached, after which blood sampling and indirect calorimetry was performed during $30 \mathrm{~min}$. Thereafter, high-dose insulin infusion was started $\left(40 \mathrm{mU} / \mathrm{m}^{2} / \mathrm{min}\right)$ for $1.5 \mathrm{~h}$ until steady state was reached, after which blood sampling and indirect calorimetry were repeated for $30 \mathrm{~min}$. During the clamp, oxygen consumption and carbon dioxide production were measured with daily calibrated automated respiratory gas analyzer using a ventilated hood system (Omnical, IDEE, Maastricht, The Netherlands). Whole body glucose and fat oxidation rates were calculated using stoichiometric equations based on measured 
oxygen consumption and carbon dioxide concentrations ${ }^{23}$ with the assumption that protein oxidation was negligible.

\section{TRACER CALCULATIONS}

Isotopic enrichment of plasma glucose was determined by electric impact ionization gas chromatography-mass spectroscopy as previously described ${ }^{24}$. Steele's single-pool steady state equations were used to calculate glucose rate of appearance $\left(R_{a}\right)$ and $R_{d}{ }^{25}$. Volume of distribution was assumed to be $0.160 \mathrm{l} / \mathrm{kg}$ for glucose. Insulin-stimulated glucose disposal (delta $R_{d}$ ) was computed as the difference between $R_{d}$ under insulin-stimulated conditions and $R_{d}$ under basal, noninsulin-stimulated conditions. Endogenous glucose production (EGP) was calculated as $R_{a}$ - exogenous glucose infusion rate. Non-oxidative glucose disposal (NOGD) was calculated as $R_{d}$ - glucose oxidation.

\section{BLOOD SAMPLE ANALYSIS}

On the 2-step hyperinsulinemic-euglycemic clamp test day, arterialized blood samples were collected and immediately centrifuged at high speed. Plasma was frozen in liquid nitrogen and stored at $-80^{\circ} \mathrm{C}$ until assayed. Plasma free fatty acids and FPG were measured with enzymatic assays automated on a Cobas Fara/Mira (FFA: Wako Nefa C test kit; Wako Chemicals, Neuss, Germany) (FPG: hexokinase method; La Roche, Basel, Switzerland). Plasma triglycerides (TG) were measured colorimetrically (Roche, Vienna, Austria). Insulin and serum liver function parameters (alanine transaminase (ALAT), aspartate aminotransferase (ASAT), $\gamma$ glutamyl transpeptidase (GGT)) were routinely measured during the screening visit and analyzed at the clinical chemistry department. 


\section{MUSCLE BIOPSY AND ANALYSIS}

Muscle biopsies were taken from the musculus vastus lateralis, prior to the 2-step hyperinsulinemic-euglycemic clamp under local anesthesia (2\% xylocaine), according to the Bergstrom technique ${ }^{26}$. Any visible non-muscle material was dissected from muscle tissue. Samples were stored at $-80^{\circ} \mathrm{C}$ until further use. In the muscle tissue obtained, lipid accumulation was assessed histochemically in crosssections using a modified oil red $\mathrm{O}$ staining for fluorescence microscopy ${ }^{27}$.

Oxidative phosphorylation (OXPHOS) was measured in whole muscle tissue by western blotting as a reflection of mitochondrial density, as previously described ${ }^{28}$. OXPHOS proteins were detected using a monoclonal antibody cocktail of 5 monoclonal antibodies directed against the different OXPHOS complexes (MS601, Mitosciences, Eugene, OR, USA). Gels were loaded with equal amounts of protein lysates to allow valid comparison. To adjust for inter-gel variation, the optical density of the band of interest per subject was normalized to the mean optical density of the complete gel. Protein content is expressed as arbitrary units (AU).

\section{STATISTICS}

All values are reported as the mean \pm standard error of the mean (SEM). Comparison of groups was performed using one-way ANOVA with Tukey (equal variances of observations) or Games-Howell (unequal variances of observations) post-hoc correction, or using Kruskal-Wallis H test with Dunn's (1964) procedure with Bonferroni correction for multiple comparison. Statistical significance was set at $P<0.05$. Normality was assessed by the Shapiro-Wilk test or determination of skewness and kurtosis. Homogeneity of variances was assessed by Levene's test for equality of variances. All statistic calculations were performed using IBM SPSS 21 (SPSS, Chicago, IL, USA). 


\section{RESULTS}

\section{SUBJECT CHARACTERISTICS}

Groups were carefully matched for BMI (table 1). NAFL subjects tended to be younger than any of the other groups $(57.6 \pm 2.5,54.5 \pm 2.0$ and $61.5 \pm 1.6$ years, $\mathrm{p}=0.055$ for CTRL, NAFL and T2DM, respectively). There was no significant difference in body composition or physical fitness (table 1). By design, FPG after an overnight fast was significantly different across groups $(5.4 \pm 0.2,5.6 \pm 0.2$ and 8.7 $\pm 0.6 \mathrm{mmol} / \mathrm{l}, \mathrm{p}<0.001$ for CTRL, NAFL and T2DM, respectively), and post-hoc analysis confirmed that FPG was significantly higher in the T2DM patients compared to the CTRL $(p<0.001)$ and NAFL $(p<0.001)$ subjects. Fasting insulin levels were significantly higher in NAFL and T2DM than in CTRL $(9.2 \pm 0.7,15.2 \pm 1.9$ and $14.9 \pm 2.6 \mathrm{mU} / \mathrm{l}, \mathrm{p}=0.012$ for CTRL, NAFL and T2DM, respectively). Plasma glucose values $120 \mathrm{~min}$ after an OGTT bolus were significantly higher in NAFL $(6.7 \pm 0.7$ $\mathrm{mmol} / \mathrm{l})$ compared to CTRL $(4.8 \pm 0.3 \mathrm{mmol} / \mathrm{l})(\mathrm{p}=0.021)$. For T2DM, no OGTT was performed. Plasma TG levels were similar across groups (table 1 ).

\section{INTRAHEPATIC LIPID CONTENT AND HEPATIC ENERGY STATUS}

By design IHL content was significantly different across groups $(1.9 \pm 0.4,9.7 \pm 2.0$ and $8.9 \pm 3.3 \%, p<0.001$ for CTRL, NAFL and T2DM, respectively). Post-hoc analysis revealed that IHL content was significantly higher in NAFL and T2DM than in CTRL, while levels in NAFL and T2DM were similar. Hepatic energy status defined as ATP/Total Phosphorus ratio did not differ between groups $(15.5 \pm 0.4,15.8 \pm 0.6$, $15.8 \pm 0.4 \%, p=0.896$ for obese controls, NAFL and T2DM, respectively).

\section{LIVER ENZYMES}

ASAT, ALAT and GGT correlated significantly with IHL content when subjects were pooled $(r=0.413, p=0.014$ for ASAT; $r=0.567, p<0.001$ for ALAT and $r=0.426, p=0.012$ for GGT). However, no significant differences were found across groups for either 
ASAT, ALAT or GGT with mean values $22.1 \pm 1.2,25.5 \pm 2.4$ and $26.5 \pm 2.0, p=0.255$ for ASAT; mean values $30.0 \pm 3.6,34.5 \pm 2.2$ and $38.3 \pm 4.2, p=0.258$ for ALAT and mean values $34.3 \pm 4.7,35.4 \pm 3.7$ and $40.6 \pm 4.9, p=0.563$ for GGT in CTRL, NAFL and T2DM, respectively (table 1 ).

Table 1. Subject characteristics.

\begin{tabular}{lcccc}
\hline Subject characteristics & CTRL & NAFL & T2DM & P value \\
\hline Subjects & 11 & 11 & 13 & \\
Age (years) & $57.6 \pm 2.5$ & $54.5 \pm 2.0$ & $61.5 \pm 1.6$ & 0.055 \\
VO2max (ml/min/kg) & $27.6 \pm 1.4$ & $26.1 \pm 1.4$ & $25.6 \pm 1.0$ & 0.492 \\
Wmax (W/kg) & $2.1 \pm 0.1$ & $1.9 \pm 0.1$ & $1.9 \pm 0.1$ & 0.272 \\
BMI (kg/m2) & $29.5 \pm 0.8$ & $30.9 \pm 0.8$ & $29.8 \pm 0.6$ & 0.384 \\
Fasting glucose (mmol/l) & $5.4 \pm 0.2$ & $5.6 \pm 0.2$ & $8.7 \pm 0.6^{\#, \$}$ & $<0.001^{*}$ \\
Insulin (mU/I) & $9.2 \pm 0.7^{\$}$ & $15.2 \pm 1.9^{\#}$ & $14.9 \pm 2.6^{\#}$ & $0.012^{*}$ \\
Glucose 120 (mmol/I) & $4.8 \pm 0.3^{\$}$ & $6.7 \pm 0.7^{\#}$ & $\mathrm{ND}$ & $0.021^{*}$ \\
IHL (\% of H2O peak) & $1.9 \pm 0.4^{\$}$ & $9.7 \pm 2.0^{\#}$ & $8.9 \pm 3.3^{\#}$ & $<0.001^{*}$ \\
ATP/Total Phosphorus (\%) & $15.5 \pm 0.4$ & $15.8 \pm 0.6$ & $15.8 \pm 0.4$ & 0.896 \\
ASAT (U/I) & $22.1 \pm 1.2$ & $25.5 \pm 2.4$ & $26.5 \pm 2.0$ & 0.255 \\
ALAT (U/I) & $30.0 \pm 3.6$ & $34.5 \pm 2.2$ & $38.3 \pm 4.2$ & 0.258 \\
Y-GT (U/I) & $34.3 \pm 4.7$ & $35.4 \pm 3.7$ & $40.6 \pm 4.9$ & 0.563 \\
Fat mass (kg) & $27.49 \pm 1.92$ & $30.70 \pm 1.15$ & $27.06 \pm 1.09$ & 0.157 \\
Fat free mass (kg) & $64.20 \pm 1.94$ & $69.00 \pm 1.98$ & $64.92 \pm 1.25$ & 0.127 \\
Visceral adipose tissue (kg) & $0.66 \pm 0.06$ & $0.81 \pm 0.06$ & $0.83 \pm 0.05$ & 0.089 \\
Fat percentage (\%) & $28.84 \pm 1.03$ & $29.95 \pm 0.70$ & $28.52 \pm 0.76$ & 0.460 \\
Triglycerides (mmol/I) & $1.26 \pm 0.17$ & $1.67 \pm 0.17$ & $1.57 \pm 0.17$ & 0.238 \\
\hline
\end{tabular}

$P$ value for group comparison (one-way ANOVA); Tukey or Games-Howell post-hoc analysis with ${ }^{\#} p<0.05$ to $C T R L ; ~ p ~ p 0.05$ to NAFL. Data are mean \pm SEM.

\section{INSULIN SENSITIVITY}

\section{ENDOGENOUS GLUCOSE PRODUCTION}

Basal EGP, reflecting hepatic glucose output, was significantly different between groups (table 2). Basal EGP was significantly higher in T2DM patients in comparison to CTRL $(p=0.002)$ and NAFL $(p<0.001)$ subjects, with the latter two being comparable $(p=0.293)$. Suppression of EGP under hyperinsulinemic conditions, expressed as the relative change in EGP upon $10 \mathrm{mU} / \mathrm{m}^{2} / \mathrm{min}$ insulin infusion (delta EGP \%), was significantly different between groups $(-70.0 \pm 3.2,-41.0 \pm 6.0,-42.1 \pm$ 
$1.9 \mu \mathrm{mol} / \mathrm{kg} / \mathrm{min}, \mathrm{p}<0.001$ for CTRL, NAFL and T2DM, respectively) (figure 1 ). Posthoc analysis revealed that EGP was suppressed more in CTRL than in the other 2 groups. Strikingly, EGP suppression was similar in NAFL and T2DM. Upon 40 $\mathrm{mU} / \mathrm{m}^{2} / \mathrm{min}$ insulin infusion, EGP was almost completely suppressed relative to baseline in all three groups (table 2).

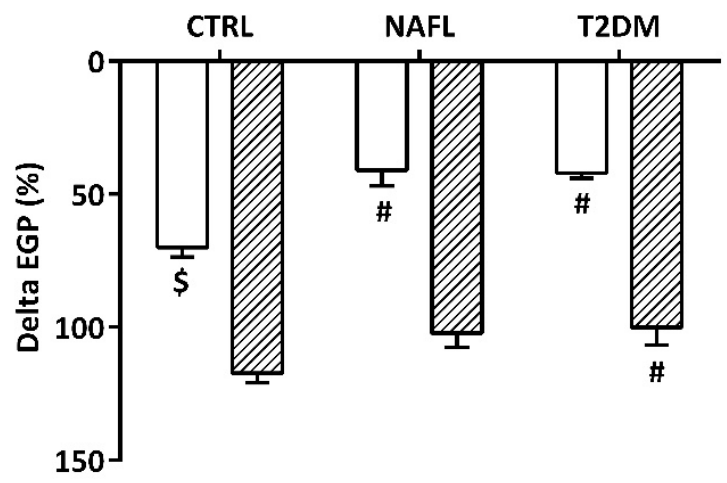

Figure 1. Insulin-stimulated suppression of endogenous glucose production (delta EGP \%) with $10 \mathrm{mU} / \mathrm{m}^{2} / \mathrm{min}$ (white columns) and 40 $\mathrm{mU} / \mathrm{m}^{2} / \mathrm{min} \quad$ (striped columns) insulin infusion. $\mathrm{n}=10$ for $C T R L, \mathrm{n}=8$ for NAFL, $\mathrm{n}=11$ for T2DM. ${ }^{*} \mathrm{p}<0.05$ to CTRL; ${ }^{\$} p<0.05$ to NAFL. Data are mean \pm SEM.

\section{INSULIN-MEDIATED GLUCOSE UPTAKE}

Basal $R_{d}$ was significantly different between groups, with basal $R_{d}$ being lowest in the groups with the lowest FPG levels, CTRL and NAFL subjects, whereas in T2DM patients with elevated FPG levels basal glucose uptake was elevated (table 2).

Insulin-stimulated glucose disposal with low-dose insulin infusion, expressed as $R_{d}$ during $10 \mathrm{mU} / \mathrm{m}^{2} / \mathrm{min}$ insulin infusion minus basal $R_{d}$, was significantly different between groups $(1.8 \pm 0.8,-0.2 \pm 0.8,-4.6 \pm 0.5 \mu \mathrm{mol} / \mathrm{kg} / \mathrm{min}, \mathrm{p}<0.001$ for CTRL, NAFL and T2DM, respectively). T2DM patients responded significantly different $(p<0.001)$ than any of the other groups, whereas no differences were detected between CTRL and NAFL subjects. In T2DM patients, this deviating effect of a lowdose insulin infusion on $R_{d}$ is generally explained by a glucose mass effect, as basal $R_{d}$ is measured under hyperglycemic conditions.

Delta $R_{d}$ with high-dose insulin infusion, expressed as $R_{d}$ during $40 \mathrm{mU} / \mathrm{m}^{2} / \mathrm{min}$ insulin infusion minus basal $R_{d}$ was significantly different between groups (22.6 \pm 
2.0, $7.7 \pm 1.7,6.1 \pm 1.0 \mu \mathrm{mol} / \mathrm{kg} / \mathrm{min}, \mathrm{p}<0.001$ for CTRL, NAFL and T2DM, respectively) (figure 2). Post-hoc analysis revealed that delta $R_{d}$ was significantly lower in NAFL and T2DM than in CTRL (both $p<0.001$ ). Strikingly, there was no differences in delta $R_{d}$ between NAFL subjects and T2DM patients $(p=0.404)$, suggesting skeletal muscle insulin sensitivity was similarly reduced in NAFL and T2DM compared to CTRL.

\section{NON-OXIDATIVE GLUCOSE DISPOSAL}

NOGD was significantly different across groups $(4.5 \pm 1.0,4.1 \pm 1.4,7.9 \pm 0.8$, $\mathrm{p}=0.021$ for CTRL, NAFL and T2DM, respectively) and was significantly higher in T2DM compared to CTRL ( $p=0.048$ ) and NAFL $(p=0.046)$ subjects (table 2$)$. T2DM patients also had the highest FPG values and basal $R_{d}$, indicating that the mass effect of glucose in T2DM patients drives basal glucose uptake in a non-oxidative manner (increased glycogen synthesis). Insulin stimulation of NOGD, measured as the increase in NOGD during $40 \mathrm{mU} / \mathrm{m}^{2} / \mathrm{min}$ insulin stimulation compared to baseline, was significantly different across groups $(13.4 \pm 2.8,3.4 \pm 1.6,0.4 \pm 1.3$ $\mu \mathrm{mol} / \mathrm{kg} / \mathrm{min}, \mathrm{p}<0.001$ for CTRL, NAFL and T2DM, respectively). Post-hoc analysis revealed that the stimulatory effect of insulin on NOGD was highest in the CTRL compared to NAFL subjects $(p=0.008)$ or to T2DM patients $(p<0.001)$. Insulinstimulated NOGD was blunted to a similar extend in NAFL subjects and T2DM patients $(p=0.478)$.

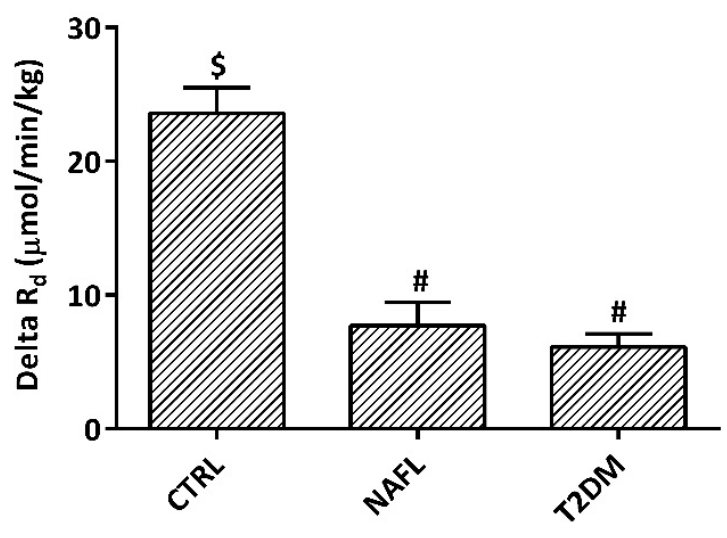

68

Figure 2. Insulin-stimulated glucose disposal (delta $R_{d}$ ) with 40 $\mathrm{mU} / \mathrm{m}^{2} / \mathrm{min}$ insulin infusion. $\mathrm{n}=10$ for CTRL, $n=8$ for NAFL, $n=11$ for T2DM. $"$ p $<0.05$ to CTRL; $\$ p<0.05$ to NAFL. Data are mean \pm SEM. 


\section{SUBSTRATE OXIDATION}

Basal glucose oxidation was not significantly different between groups (table 2). Insulin stimulation of glucose oxidation, measured as the increase in glucose oxidation during $40 \mathrm{mU} / \mathrm{m}^{2} / \mathrm{min}$ insulin infusion compared to baseline, was significantly different between groups $(8.9 \pm 0.4,5.8 \pm 0.9,5.7 \pm 0.7 \mu \mathrm{mol} / \mathrm{kg} / \mathrm{min}$, $\mathrm{p}=0.005$ for CTRL, NAFL and T2DM, respectively). Post-hoc analysis revealed that insulin-stimulated glucose oxidation was highest in CTRL $(p=0.049$ compared to NAFL and $p=0.006$ compared to T2DM), whereas this stimulation was similarly blunted ( $p=0.999$ ) in NAFL and T2DM (table 2).

Basal fat oxidation was not significantly different between groups (table 2). Suppression of fat oxidation by insulin was significantly different between groups with both low-dose $(-1.3 \pm 0.3,-0.4 \pm 0.2,-0.8 \pm 0.2 \mu \mathrm{mol} / \mathrm{kg} / \mathrm{min}, \mathrm{p}=0.044$ for CTRL, NAFL and T2DM, respectively) and high-dose $(-2.3 \pm 0.2,-1.5 \pm 0.2,-1.7 \pm 0.2$ $\mu \mathrm{mol} / \mathrm{kg} / \mathrm{min}, \mathrm{p}=0.021$ for CTRL, NAFL and T2DM, respectively) insulin infusion. Post-hoc analysis revealed that the inhibitory effect of insulin on fat oxidation was most pronounced in CTRL subjects at both rates of insulin infusion. Both NAFL and T2DM had lost their capacity to reduce fat oxidation rates by insulin to a similar extent, although the insulin-mediated suppression of fat oxidation during high-dose insulin stimulation tended to be less in the NAFL subjects compared to the T2DM patients ( $p=0.065)$ (table 2$)$.

\section{PLASMA FREE FATTY ACIDS}

Plasma FFA levels after an overnight fast were comparable across groups (table 2). Adipose tissue insulin sensitivity was measured as insulin-mediated suppression of plasma FFA concentrations on a low- and a high-dose infusion of insulin (table 2). There was a significant difference in the suppression of plasma FFA concentration with low-dose insulin infusion between groups $(-69.6 \pm 3.3,-54.5 \pm 5.0,-59.1 \pm 3.0$ $\%, p=0.030$ for CTRL, NAFL and T2DM, respectively) (figure 3). Post-hoc analysis revealed that CTRL subjects were most sensitive to the inhibitory effect of insulin on adipose tissue lipolysis, whereas no differences were observed between NAFL subjects and T2DM patients. These observations were not made for the high-dose insulin infusion (table 2) (figure 3). 
Table 2. Substrate kinetics.

\begin{tabular}{|c|c|c|c|c|}
\hline & CTRL & NAFL & T2DM & $P$ value \\
\hline \multicolumn{5}{|c|}{$\mathrm{EGP}(\mu \mathrm{mol} / \mathrm{min} / \mathrm{kg})$} \\
\hline Basal & $10.1 \pm 0.4$ & $9.6 \pm 0.2$ & $12.5 \pm 0.5^{\#, \$}$ & $<0.001$ \\
\hline $10 \mathrm{mU}$ & $3.1 \pm 0.4^{\$}$ & $5.6 \pm 0.6^{\#}$ & $7.2 \pm 0.3^{\#, \$}$ & $<0.001$ \\
\hline Delta \% & $-70.0 \pm 3.2^{\$}$ & $-41.0 \pm 6.0^{\#}$ & $-42.1 \pm 1.9^{\#}$ & $<0.001$ \\
\hline $40 \mathrm{mU}$ & $-1.9 \pm 0.4$ & $-0.2 \pm 0.5$ & $0.0 \pm 0.7$ & 0.047 \\
\hline Delta \% & $-119.9 \pm 4.1$ & $-102.4 \pm 5.2$ & $-100.0 \pm 6.7^{\#}$ & 0.034 \\
\hline \multicolumn{5}{|c|}{$R_{d}(\mu \mathrm{mol} / \mathrm{min} / \mathrm{kg})$} \\
\hline Basal & $10.3 \pm 0.4$ & $9.8 \pm 0.2$ & $12.7 \pm 0.5^{\#, \$}$ & $<0.001$ \\
\hline $10 \mathrm{mU}$ & $12.1 \pm 0.8^{\$}$ & $9.6 \pm 0.8^{\#}$ & $8.1 \pm 0.3^{\#}$ & $<0.001$ \\
\hline Delta & $1.8 \pm 0.8$ & $-0.2 \pm 0.8$ & $-4.6 \pm 0.5^{\#, \$}$ & $<0.001$ \\
\hline $40 \mathrm{mU}$ & $32.9 \pm 2.0^{\$}$ & $17.5 \pm 1.8^{\#}$ & $18.8 \pm 1.1^{\#}$ & $<0.001$ \\
\hline Delta & $22.6 \pm 2.0^{\$}$ & $7.7 \pm 1.7^{\#}$ & $6.1 \pm 1.0^{\#}$ & $<0.001$ \\
\hline \multicolumn{5}{|c|}{ Glucose $0 x .(\mu \mathrm{mol} / \mathrm{min} / \mathrm{kg})$} \\
\hline Basal & $5.6 \pm 1.3$ & $5.5 \pm 1.3$ & $4.8 \pm 0.7$ & 0.807 \\
\hline $10 \mathrm{mU}$ & $8.8 \pm 1.0$ & $5.8 \pm 1.0$ & $6.6 \pm 0.7$ & 0.067 \\
\hline Delta & $3.9 \pm 1.2$ & $0.4 \pm 1.0$ & $1.8 \pm 0.6$ & 0.057 \\
\hline $40 \mathrm{mU}$ & $14.5 \pm 1.6$ & $10.8 \pm 1.3$ & $10.5 \pm 0.6^{\#}$ & 0.041 \\
\hline Delta & $8.9 \pm 0.4^{\$}$ & $5.8 \pm 0.9^{\#}$ & $5.7 \pm 0.7^{\#}$ & 0.005 \\
\hline \multicolumn{5}{|c|}{ NOGD $(\mu \mathrm{mol} / \mathrm{min} / \mathrm{kg})$} \\
\hline Basal & $4.5 \pm 1.0$ & $4.1 \pm 1.4$ & $7.9 \pm 0.8^{\#, \$}$ & 0.021 \\
\hline $10 \mathrm{mU}$ & $2.8 \pm 1.0$ & $3.8 \pm 0.6$ & $1.5 \pm 0.6$ & 0.129 \\
\hline Delta & $-2.3 \pm 1.5$ & $0.1 \pm 1.2$ & $-6.4 \pm 0.7^{\#, \$}$ & 0.002 \\
\hline $40 \mathrm{mU}$ & $17.7 \pm 1.9 \$$ & $6.5 \pm 1.3^{\#}$ & $8.3 \pm 1.0^{\#}$ & $<0.001$ \\
\hline Delta & $13.4 \pm 2.8^{\$}$ & $3.4 \pm 1.6^{\#}$ & $0.4 \pm 1.3^{\#}$ & $<0.001$ \\
\hline \multicolumn{5}{|c|}{ Fat Ox. $(\mu \mathrm{mol} / \mathrm{min} / \mathrm{kg})$} \\
\hline Basal & $3.7 \pm 0.2$ & $3.4 \pm 0.3$ & $3.9 \pm 0.2$ & 0.329 \\
\hline $10 \mathrm{mU}$ & $2.7 \pm 0.3$ & $3.0 \pm 0.3$ & $3.1 \pm 0.2$ & 0.528 \\
\hline Delta & $-1.3 \pm 0.3^{\$}$ & $-0.4 \pm 0.2^{\#}$ & $-0.8 \pm 0.2$ & 0.044 \\
\hline $40 \mathrm{mU}$ & $1.6 \pm 0.3$ & $2.0 \pm 0.3$ & $2.2 \pm 0.2$ & 0.338 \\
\hline Delta & $-2.3 \pm 0.2^{\$}$ & $-1.5 \pm 0.2^{\#}$ & $-1.7 \pm 0.2$ & 0.021 \\
\hline \multicolumn{5}{|c|}{ Plasma FFA ( $\mu \mathrm{mol} / \mathrm{l})$} \\
\hline Basal & $657.6 \pm 46.7$ & $726.1 \pm 54.5$ & $674.9 \pm 27.7$ & 0.533 \\
\hline $10 \mathrm{mU}$ & $193.4 \pm 17.6^{\$}$ & $317.4 \pm 33.2^{\#}$ & $271.7 \pm 19.0$ & 0.004 \\
\hline Delta \% & $-69.6 \pm 3.3^{\$}$ & $-54.5 \pm 5.0^{\#}$ & $-59.1 \pm 3.0$ & 0.030 \\
\hline $40 \mathrm{mU}$ & $98.3 \pm 13.5$ & $149.4 \pm 21.3$ & $144.6 \pm 18.7$ & 0.106 \\
\hline Delta \% & $-85.3 \pm 1.4$ & $-80.0 \pm 1.7$ & $-78.6 \pm 2.6$ & 0.056 \\
\hline
\end{tabular}

$\mathrm{P}$ value for group comparison (one-way ANOVA); Tukey or Games-Howell post-hoc analysis with ${ }^{*} p<0.05$ to $C T R L ;{ }^{\$} p<0.05$ to NAFL. Data are mean \pm SEM. 


\section{SKELETAL MUSCLE LIPID CONTENT}

Lipid/cell area was determined in 10 CTRL, 9 NAFL and 13 T2DM participants. There was no significant difference in lipid/cell area in type I muscle fibers $(2.21 \pm 0.47$, $3.66 \pm 0.75$ and $2.99 \pm 0.37 \%, p=0.331$ for CTRL, NAFL and T2DM, respectively). Also in type II muscle fibers lipid/cell area was not significantly different across groups $(1.36 \pm 0.30,1.74 \pm 0.36$ and $1.82 \pm 0.20 \%, p=0.190$ in CTRL, NAFL and T2DM, respectively).

\section{MITOCHONDRIAL DENSITY}

Mitochondrial density was evaluated by measuring protein content of 5 structural subunits of the 5 distinct complexes of the electron transport chain. Neither the individual, nor averaging the protein content of these complexes revealed significant difference in mitochondrial density between CTRL, NAFL and T2DM patients (table 3).

\section{DISCUSSION}

NAFL has been associated with obesity and insulin resistance ${ }^{3,6-12}$. Moreover, deterioration of metabolic abnormalities over time is more prevalent and more severe in obese subjects with NAFL than in lean subjects without NAFL ${ }^{19}$. Fabbrini et al. ${ }^{8}$ showed that obese people with high IHL content are more insulin resistant than obese people with low IHL content, and Magkos et al..$^{29}$ demonstrated that an increase in whole body adiposity without a concomitant increase in $\mathrm{HL}$ content is not associated with augmented metabolic dysfunction. It is not known, however, how severe the metabolic aberrations in obese subjects with NAFL are relative to patients with overt T2DM. We hypothesized that metabolic aberrations in obese subjects with NAFL would be intermediate to values observed for overweight/obese CTRL subjects and T2DM patients. In contrast to this hypothesis, we demonstrate that under conditions of insulin stimulation hepatic, skeletal muscle and adipose tissue insulin sensitivity all are impaired in NAFL subjects to a 
similar degree than in patients with overt T2DM. This striking observation underscores that NAFL in obese and normoglycemic subjects should be viewed as a serious health threat that requires early diagnosis and treatment.

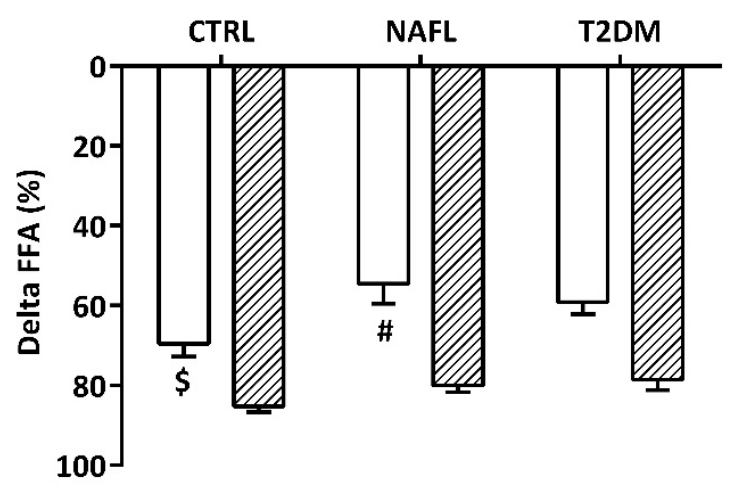

Figure 3. Insulin-stimulated suppression of plasma free fatty acids (delta FFA \%) with $10 \mathrm{mU} / \mathrm{m}^{2} / \mathrm{min}$ (white columns) and $40 \mathrm{mU} / \mathrm{m}^{2} / \mathrm{min}$ (striped columns) insulin infusion. $\mathrm{n}=10$ for $\mathrm{CTRL}, \mathrm{n}=8$ for NAFL, $\mathrm{n}=11$ for T2DM. ${ }^{\#} \mathrm{p}<0.05$ to $\mathrm{CTRL} ;{ }^{\$} \mathrm{p}<0.05$ to NAFL. Data are mean \pm SEM.

In accordance with previous observations, NAFL subjects were not characterized by fasting hyperglycemia. Nevertheless, they possessed elevated fasting insulin and glucose values at the end of an OGTT measurement, indicative of insulin resistance. Non-insulin-stimulated EGP, $R_{d}$, glucose oxidation and NOGD were comparable in NAFL subjects to CTRL subjects. This underscores that to unveil the metabolic aberrations in obese patients with NAFL challenged conditions like an OGTT or a 2step hyperinsulinemic-euglycemic clamp are warranted. Interestingly, fasting insulin levels in NAFL patients were as high as observed for patients with overt T2DM. Thus the elevated insulin levels in the NAFL subjects may suffice to mask deteriorations in glucose metabolism that are already present in NAFL.

Although it has long been recognized that elevated liver enzymes precede the development of T2DM ${ }^{17,18}$, it should be noted that despite an average hepatic fat content of $9.7 \%$, the NAFL subjects in the present study had liver enzymes that were not different from the CTRL subjects and were well within the normal physiological range. This is in line with previous cohort studies demonstrating that plasma liver enzymes are within normal limits in nearly $80 \%$ of patients with $\mathrm{NAFL}^{30}$. Thus, simply using circulatory liver enzymes as a diagnostic marker for NAFL will result in underestimation of NAFL and its metabolic corollaries. 
NAFL might predict T2DM ${ }^{31}$, with NAFL preceding metabolic disorders ${ }^{31}$. Furthermore, it has been described that the prevalence of IFG, IGT and T2DM is considerably more common in obese people with NAFL ${ }^{32,33}$. In our NAFL subjects, we found that hepatic, skeletal muscle and adipose tissue insulin sensitivity was impaired to a similar extent as in our patients with overt T2DM. Given that metabolic aberrations, if not treated, progress with age, it is important to note that the severity of the metabolic aberrations observed in NAFL subjects reach the severity of those observed with full blown T2DM, despite the tendency of a lower age in our NAFL subjects compared to the T2DM patients $(54.5 \pm 2.04$ vs. $61.5 \pm$ 1.62 years). Also this observation underscores the need to start treating people with NAFL promptly upon diagnosis.

Table 3. Mitochondrial density protein content (AU).

\begin{tabular}{lcccc}
\hline & CTRL & NAFL & T2DM & P value \\
\hline Complex I & $0.96 \pm 0.13$ & $0.96 \pm 0.12$ & $0.87 \pm 0.10$ & 0.828 \\
Complex II & $0.96 \pm 0.10$ & $0.94 \pm 0.09$ & $0.96 \pm 0.10$ & 0.986 \\
Complex III & $1.03 \pm 0.08$ & $1.00 \pm 0.11$ & $0.90 \pm 0.06$ & 0.501 \\
Complex IV & $1.01 \pm 0.08$ & $0.98 \pm 0.08$ & $0.91 \pm 0.06$ & 0.645 \\
Complex V & $1.06 \pm 0.06$ & $0.93 \pm 0.08$ & $0.89 \pm 0.05$ & 0.210 \\
Average of the complexes & $1.02 \pm 0.07$ & $0.97 \pm 0.08$ & $0.90 \pm 0.05$ & 0.487 \\
\hline
\end{tabular}

$\mathrm{P}$ value for group comparison (one-way ANOVA); Tukey or Games-Howell post-hoc analysis with " $p<0.05$ to CTRL; ${ }^{\$} p<0.05$ to NAFL. Data are mean \pm SEM.

Another characteristic of the insulin resistant skeletal muscle is impaired metabolic flexibility ${ }^{28}$. Although metabolic inflexibility might reflect reduced ability of mitochondria to shift fuel selection, it also reflects reduced insulin-stimulated glucose uptake, and hence reduced availability of glucose for oxidation ${ }^{34}$. Upon insulin stimulation, changes in glucose and lipid oxidation were similar in NAFL subjects and T2DM patients. As a consequence, metabolic flexibility in NAFL subjects and T2DM patients was similarly impaired. Not only impaired glucose oxidation was responsible for the impaired insulin-stimulated rate of glucose disappearance, also NOGD was similarly impaired in NAFL subjects and T2DM patients compared to CTRL subjects. Previously, we reported intermediate values for carbohydrate oxidation and NOGD in a group of first degree relatives, when compared with age- and BMI-matched control and T2DM patients ${ }^{35}$. Furthermore, in another group of 'pre-diabetic' subjects (IFG or IGT), metabolic flexibility was found to be reduced, but not NOGD, when compared to normal glucose tolerant 
subjects $^{36}$. Therefore, the similar reduction in $R_{d}$, metabolic flexibility and NOGD in NAFL subjects as in T2DM patients was unexpected.

We conclude that NAFL subjects develop hepatic, skeletal muscle and adipose tissue insulin resistance to a similar extent as patients of similar to older age with well-established T2DM. Therefore, in people with NAFL, early diagnosis and proper treatment of insulin resistance is necessary to prevent development of type 2 diabetes mellitus. 


\section{REFERENCES}

1. Gastaldelli, A. Role of beta-cell dysfunction, ectopic fat accumulation and insulin resistance in the pathogenesis of type 2 diabetes mellitus. Diabetes Res Clin Pract 93 Suppl 1, S60-65 (2011).

2. Abdul-Ghani, M.A. \& DeFronzo, R.A. Pathophysiology of prediabetes. Curr Diab Rep 9, 193-199 (2009).

3. Yki-Jarvinen, H. Liver fat in the pathogenesis of insulin resistance and type 2 diabetes. Dig Dis 28, 203-209 (2010).

4. Bilet, L., et al. Acute exercise does not decrease liver fat in men with overweight or NAFLD. Sci Rep 5, 9709 (2015).

5. Lindeboom, L., et al. Proton magnetic resonance spectroscopy reveals increased hepatic lipid content after a single high-fat meal with no additional modulation by added protein. Am J Clin Nutr 101, 65-71 (2015).

6. Utzschneider, K.M. \& Kahn, S.E. Review: The role of insulin resistance in nonalcoholic fatty liver disease. J Clin Endocrinol Metab 91, 4753-4761 (2006).

7. Deivanayagam, S., et al. Nonalcoholic fatty liver disease is associated with hepatic and skeletal muscle insulin resistance in overweight adolescents. Am J Clin Nutr 88, 257-262 (2008).

8. Fabbrini, E., et al. Intrahepatic fat, not visceral fat, is linked with metabolic complications of obesity. Proc Natl Acad Sci U S A 106, 15430-15435 (2009).

9. Korenblat, K.M., Fabbrini, E., Mohammed, B.S. \& Klein, S. Liver, muscle, and adipose tissue insulin action is directly related to intrahepatic triglyceride content in obese subjects. Gastroenterology 134, 1369-1375 (2008).

10. Kotronen, A., Juurinen, L., Tiikkainen, M., Vehkavaara, S. \& Yki-Jarvinen, H. Increased liver fat, impaired insulin clearance, and hepatic and adipose tissue insulin resistance in type 2 diabetes. Gastroenterology 135, 122-130 (2008).

11. Seppala-Lindroos, A., et al. Fat accumulation in the liver is associated with defects in insulin suppression of glucose production and serum free fatty acids independent of obesity in normal men. J Clin Endocrinol Metab 87, 3023-3028 (2002).

12. Tiikkainen, M., et al. Liver-fat accumulation and insulin resistance in obese women with previous gestational diabetes. Obes Res 10, 859-867 (2002). 
13. Byrne, C.D., Olufadi, R., Bruce, K.D., Cagampang, F.R. \& Ahmed, M.H. Metabolic disturbances in non-alcoholic fatty liver disease. Clin Sci (Lond) 116, 539-564 (2009).

14. Kotronen, A., Westerbacka, J., Bergholm, R., Pietilainen, K.H. \& YkiJarvinen, H. Liver fat in the metabolic syndrome. J Clin Endocrinol Metab 92, 3490-3497 (2007).

15. Marchesini, G., et al. Association of nonalcoholic fatty liver disease with insulin resistance. Am J Med 107, 450-455 (1999).

16. Perseghin, G., et al. Elevated fasting plasma C-peptide occurs in nondiabetic individuals with fatty liver, irrespective of insulin resistance. Diabet Med 26, 847-854 (2009).

17. Andre, P., et al. Hepatic markers and development of type 2 diabetes in middle aged men and women: a three-year follow-up study. The D.E.S.I.R. Study (Data from an Epidemiological Study on the Insulin Resistance syndrome). Diabetes Metab 31, 542-550 (2005).

18. Fraser, A., Thinggaard, M., Christensen, K. \& Lawlor, D.A. Alanine aminotransferase, gamma-glutamyltransferase (GGT) and all-cause mortality: results from a population-based Danish twins study alanine aminotransferase, GGT and mortality in elderly twins. Liver Int 29, 14941499 (2009).

19. Fan, J.G., et al. Effects of nonalcoholic fatty liver disease on the development of metabolic disorders. J Gastroenterol Hepatol 22, 10861091 (2007).

20. Kuipers, H., Verstappen, F.T., Keizer, H.A., Geurten, P. \& van Kranenburg, G. Variability of aerobic performance in the laboratory and its physiologic correlates. Int J Sports Med 6, 197-201 (1985).

21. DeFronzo, R.A., Tobin, J.D. \& Andres, R. Glucose clamp technique: a method for quantifying insulin secretion and resistance. Am J Physiol 237, E214-223 (1979).

22. Mensink, M., Blaak, E.E., van Baak, M.A., Wagenmakers, A.J. \& Saris, W.H. Plasma free Fatty Acid uptake and oxidation are already diminished in subjects at high risk for developing type 2 diabetes. Diabetes 50, 2548-2554 (2001).

23. Frayn, K.N. Calculation of substrate oxidation rates in vivo from gaseous exchange. J Appl Physiol Respir Environ Exerc Physiol 55, 628-634 (1983). 
24. Ackermans, M.T., et al. The quantification of gluconeogenesis in healthy men by (2) $\mathrm{H} 2 \mathrm{O}$ and [2-(13)C]glycerol yields different results: rates of gluconeogenesis in healthy men measured with (2) H2O are higher than those measured with [2-(13)C]glycerol. The Journal of clinical endocrinology and metabolism 86, 2220-2226 (2001).

25. Steele, R. Influences of glucose loading and of injected insulin on hepatic glucose output. Ann N Y Acad Sci 82, 420-430 (1959).

26. Bergstrom, J., Hermansen, L., Hultman, E. \& Saltin, B. Diet, muscle glycogen and physical performance. Acta Physiol Scand 71, 140-150 (1967).

27. Koopman, R., Schaart, G. \& Hesselink, M.K. Optimisation of oil red O staining permits combination with immunofluorescence and automated quantification of lipids. Histochem Cell Biol 116, 63-68 (2001).

28. Meex, R.C., et al. Restoration of muscle mitochondrial function and metabolic flexibility in type 2 diabetes by exercise training is paralleled by increased myocellular fat storage and improved insulin sensitivity. Diabetes 59, 572-579 (2010).

29. Magkos, F., Fabbrini, E., Mohammed, B.S., Patterson, B.W. \& Klein, S. Increased whole-body adiposity without a concomitant increase in liver fat is not associated with augmented metabolic dysfunction. Obesity (Silver Spring) 18, 1510-1515 (2010).

30. Browning, J.D., et al. Prevalence of hepatic steatosis in an urban population in the United States: impact of ethnicity. Hepatology 40, 1387-1395 (2004).

31. Lattuada, G., Ragogna, F. \& Perseghin, G. Why does NAFLD predict type 2 diabetes? Curr Diab Rep 11, 167-172 (2011).

32. Ortiz-Lopez, C., et al. Prevalence of prediabetes and diabetes and metabolic profile of patients with nonalcoholic fatty liver disease (NAFLD). Diabetes care 35, 873-878 (2012).

33. Yun, J.W., et al. Abnormal glucose tolerance in young male patients with nonalcoholic fatty liver disease. Liver Int 29, 525-529 (2009).

34. Galgani, J.E., Moro, C. \& Ravussin, E. Metabolic flexibility and insulin resistance. Am J Physiol Endocrinol Metab 295, E1009-1017 (2008).

35. Phielix, E., et al. Lower intrinsic ADP-stimulated mitochondrial respiration underlies in vivo mitochondrial dysfunction in muscle of male type 2 diabetic patients. Diabetes 57, 2943-2949 (2008). 
36. Faerch, K. \& Vaag, A. Metabolic inflexibility is a common feature of impaired fasting glycaemia and impaired glucose tolerance. Acta Diabetol 48, 349-353 (2011). 




\section{CHAPTER 4}

\section{ACUTE EXERCISE DOES NOT DECREASE LIVER FAT IN MEN WITH OVERWEIGHT OR NAFLD}

Bram Brouwers*, Lena Bilet*, Petronella A. van Ewijk, Matthijs K.C. Hesselink,

Marianne E. Kooi, Patrick Schrauwen, Vera B. Schrauwen-Hinderling

Published in Scientific Reports, 2015, 5:9709

* These authors contributed equally to this work 


\section{ABSTRACT}

Elevated hepatic lipid content (intrahepatic lipid, IHL) increases the risk of metabolic complications. Although prolonged exercise training lowers IHL, it is unknown if acute exercise has the same effect. Furthermore, hepatic ATP content may be related to insulin resistance and IHL. We aimed to investigate if acute exercise leads to changes in IHL and whether this is accompanied by changes in hepatic ATP.

Twenty-one men (age $54.8 \pm 7.2$ years, BMI $29.7 \pm 2.2 \mathrm{~kg} / \mathrm{m}^{2}$ ) performed a $2 \mathrm{~h}$ cycling protocol, once while staying fasted and once while ingesting glucose. $\mathrm{IHL}$ was determined at baseline, $30 \mathrm{~min}$ post-exercise and $4 \mathrm{~h}$ post-exercise. Additionally ATP/Total Phosphorus ratio was measured at baseline and $4 \mathrm{~h}$ post-exercise.

Compared with baseline values we did not observe any statistically significant changes in $\mathrm{IHL}$ within $30 \mathrm{~min}$ post-exercise in neither the fasted nor the glucosesupplemented condition. However, IHL was elevated $4 \mathrm{~h}$ post-exercise compared with baseline in the fasted condition (from $8.3 \pm 1.8$ to $8.7 \pm 1.8 \%, p=0.010$ ), an effect that was blunted by glucose supplementation (from $8.3 \pm 1.9$ to $8.3 \pm 1.9 \%$, $\mathrm{p}=0.789$ ).

In conclusion, acute exercise does not decrease liver fat in overweight middle-aged men. Moreover, $\mathrm{IHL}$ increased $4 \mathrm{~h}$ post-exercise in the fasted condition, an increase that was absent in the glucose-supplemented condition. These data suggest that a single bout of exercise may not be able to lower IHL. 


\section{INTRODUCTION}

Regular exercise has beneficial effects on metabolic risk factors associated with type 2 diabetes ${ }^{1,2}$. Recent studies have suggested that prolonged exercise training reduces liver fat content and may thereby contribute to the beneficial effects of exercise on metabolic risk ${ }^{3-6}$. Results from exercise training studies in animals revealed that the effect of exercise on hepatic lipid content (intrahepatic lipid, IHL) strongly depends on the diet and that exercise is more effective in reducing $\mathrm{IHL}$ under conditions that favor liver fat accretion, such as when animals are fed a highfat diet $^{7}$. Interestingly, human data also revealed that exercise training appears to be more potent in reducing $\mathrm{IHL}$ in subjects with increased baseline IHL. Thus, the exercise-mediated reduction in IHL is more pronounced in subjects with NAFLD, type 2 diabetes, or in elderly ${ }^{8}$ than in healthy normal weight and young subjects. Furthermore, like with exercise training, acute bouts of exercise also improve insulin sensitivity ${ }^{9-11}$. The acute effects of exercise on IHL, however, have not yet been intensively studied. As yet, only three studies have examined the effect of acute exercise on IHL. However, all three of these studies were performed in lean young healthy volunteers in which liver fat is usually rather low ${ }^{12-14}$, which would complicate detecting exercise-mediated reductions in IHL. To circumvent this, one study ${ }^{12}$ let the male subjects consume a high-fat diet or a mixed diet for $67 \mathrm{~h}$ before exercise. Subsequently IHL was measured (with ${ }^{1} \mathrm{H}-\mathrm{MRS}$ ) before and after $90 \mathrm{~min}$ of moderate-intensity cycling. No statistically significant decrease was found directly after exercise, irrespective of the dietary condition. Hence it was concluded that acute exercise does not lower liver fat in young healthy men. In the other two studies, one study including male subjects with an average age of 28.9 years old ${ }^{13}$ and one study including male and female subjects with a slightly older age of 37.6 years old ${ }^{14}$, subjects also consumed a high-fat diet 3 days prior to the exercise trial. Here IHL (measured with ${ }^{1} \mathrm{H}-\mathrm{MRS}$ ) was increased rather than decreases after a $2 \mathrm{~h}$ exercise bout (50-60\% of $\mathrm{VO}_{2 \max }$ ), and it was speculated that the increase in $\mathrm{IHL}$ upon exercise was due to increased free fatty acid (FFA) availability during exercise. A clinically more relevant question, however, would be if acute exercise lowers liver fat in a middle-aged overweight subject population who are prone to the development of fatty liver or may already have elevated IHL (NAFLD), but such information is presently lacking. 
As mentioned above, a potentially biasing complication when measuring IHL postexercise is the increase in plasma fatty acids (FA) that goes along with exercise in the fasted state (originating from exercise-mediated increase in adipose tissue lipolysis) $)^{15,16}$. Plasma FA is an important source for hepatic triglyceride ${ }^{17}$, and therefore high levels of plasma FA during exercise might mask a potential exerciselowering effect. To circumvent this bias, we here investigated the effect of acute exercise on $\mathrm{IHL}$ in middle-aged overweight sedentary subjects with a wide range of liver fat content under conditions with high and low plasma FA concentrations.

Next to IHL, hepatic ATP concentrations, a measure for liver energy status, have also been suggested to be related to insulin resistance and hepatic lipid accumulation ${ }^{18}$. Therefore, we also studied if changes in $\mathrm{IHL}$ are accompanied by changes in hepatic ATP concentration. To this end, we employed ${ }^{1} \mathrm{H}$-MRS to determine IHL before and after exercise performed with and without glucose ingestion to suppress FFA levels, as well as $4 \mathrm{~h}$ post-exercise. ${ }^{31} \mathrm{P}$-MRS was employed before exercise and $4 \mathrm{~h}$ post-exercise to determine hepatic ATP concentrations.

\section{RESEARCH DESIGN AND METHODS}

\section{SUBJECTS}

Twenty-one middle-aged overweight men with a wide range of liver fat content participated in this study. None of the subjects participated in competitive sports and subjects with unstable body weight (> $3 \mathrm{~kg}$ change in preceding six months) were excluded from the study. The study protocol conforms to the ethical guidelines of the 1975 Declaration of Helsinki as reflected in a priori approval by the institution's human research committee and written informed consent was obtained from all participants. 


\section{STUDY PROTOCOL}

Before the start of the study, body composition and maximal aerobic capacity $\left(\mathrm{VO}_{2 \max }\right)$ were determined in all subjects. The experimental trial comprised two separate test days separated by at least one week and performed in random order. Subjects refrained from physical activity two days prior to the test days. Furthermore, subjects were instructed to consume a standardized meal the evening prior to the test days and stayed fasted from 10 P.M. onwards. On the test days, subjects reported to the laboratory (Maastricht University Medical Center +) at 7:00 A.M. after an overnight fast. IHL was investigated by proton magnetic resonance spectroscopy $\left({ }^{1} \mathrm{H}-\mathrm{MRS}\right)$. After this, a Teflon cannula was inserted into an antecubital forearm vein for sampling of blood and subjects rested for $30 \mathrm{~min}$. Immediately after drawing the first blood sample, subjects ingested either $1.4 \mathrm{~g} / \mathrm{kg}$ body weight of glucose (dissolved in water to a $20 \%$ solution and flavoured with 1 $\mathrm{ml}$ lemon juice) or the same amount of water. After this, subjects started exercising ( $t=0 \mathrm{~min}$ ) on a stationary bike at $50 \%$ of their pre-determined maximal power output $\left(\mathrm{W}_{\max }\right)$ for $2 \mathrm{~h}$. During exercise, blood samples were drawn and substrate oxidation was measured by indirect calorimetry (Omnical, Maastricht University, Maastricht, The Netherlands) every 30min (at t=30, 60, 90 and 120min) while heart rate was constantly measured. Immediately after cessation of exercise ( $t=120 \mathrm{~min})$, subjects were transferred to the MR facility for a second ${ }^{1} \mathrm{H}$-MRS measurement. Importantly, due to the transfer time and the preparation steps of the ${ }^{1} \mathrm{H}-\mathrm{MRS}$ measurement itself, IHL could only be measured within 30min after cessation of exercise. Subsequently, subjects bed-rested for $3 \mathrm{~h}$, followed by a third ${ }^{1} \mathrm{H}-\mathrm{MRS}$ scan $4 \mathrm{~h}$ post-exercise. Additionally, in a subgroup of eight subjects, ATP/Total Phosphorus ( $P$ ) ratio was measured by phosphorous magnetic resonance spectroscopy ( $\left.{ }^{31} \mathrm{P}-\mathrm{MRS}\right)$ at baseline as well as $4 \mathrm{~h}$ post-exercise. During the $4 \mathrm{~h}$ postexercise period, blood samples were drawn and substrate oxidation was measured for $15 \mathrm{~min}$ every hour (at $\mathrm{t}=180,240,300$ and $360 \mathrm{~min}$ ) in all subjects. The experimental design is depicted in figure 1. 


\section{MEASUREMENTS PRIOR TO TEST DAYS}

A routine incremental cycling test on a stationary bike was used to determine maximal exercise capacity as described previously ${ }^{19}$ and a hydrostatic weighing with simultaneous measurement of lung volume was used to determine body composition. The equation of $\mathrm{Siri}^{20}$ was used to calculate fat percentage, fat mass and fat-free mass.

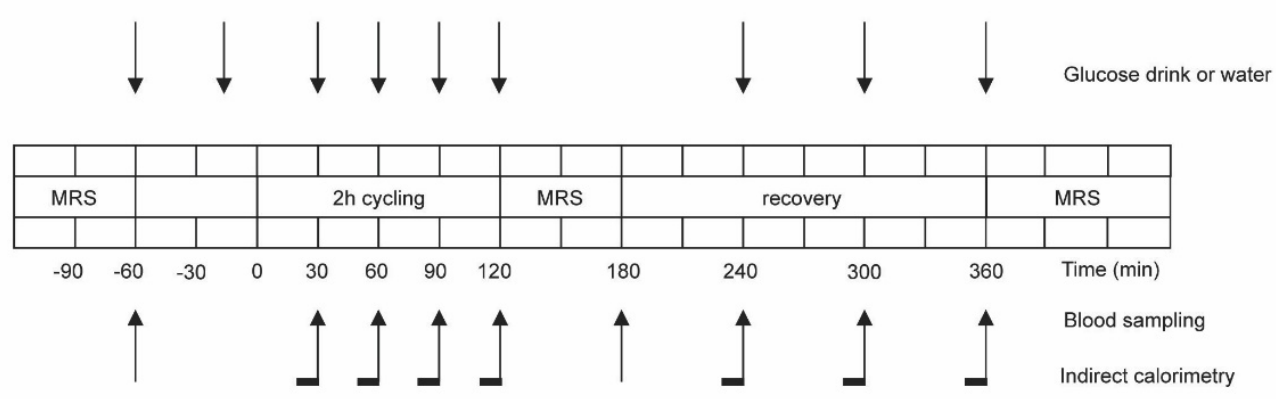

Figure 1. The experimental design of the study. All subjects performed the protocol twice, one time in the fasted state consuming water and one time with glucose supplementation. MRS: magnetic resonance spectroscopy.

\section{BLOOD SAMPLE ANALYSIS}

Blood samples were collected in EDTA-containing tubes and immediately spun at high speed and frozen in liquid nitrogen and subsequently stored at $-80{ }^{\circ} \mathrm{C}$ until assayed. Plasma free fatty acids, triglycerides, and glucose were measured with enzymatic assays automated on a Cobas Fara/Mira (FFA: Wako Nefa C test kit; Wako Chemicals, Neuss, Germany) (glucose: hexokinase method; Roche, Basel, Switzerland) (TG: ABX Pentra CP reagents, Horiba ABX Diagnostics, Montpellier, France) (glycerol: Enzytec ${ }^{\mathrm{TM}}$ glycerol kit, R-Biopharm, Germany). The liver enzymes Gamma-GT, ASAT and ALAT were routinely measured during the screening visit and analysed in the clinical chemistry department in the hospital. 


\section{PROTON MAGNETIC RESONANCE SPECTROSCOPY}

$\mathrm{IHL}$ was determined in vivo by ${ }^{1} \mathrm{H}-\mathrm{MRS}$. Measurements were performed on a whole body scanner (1.5T, Intera, Philips Healthcare, Best, The Netherlands) as reported previously ${ }^{21}$. An $18 \mathrm{~cm}^{3}$ Volume of Interest (VOI) was placed within the lower right hepatic lobe (point resolved spectroscopy (PRESS), repetition time $(T R)=4 \mathrm{~s}$, Echo time $(T E)=23 \mathrm{~ms}$, bandwidth $(B W)=1000 \mathrm{~Hz}, \mathrm{n}=1024$ points, number of signal averages $(N S A)=64)$. To minimize motion artifacts, subjects were asked to breathe in the rhythm of the measurement and to be at end-expiration during acquisition of spectra. Water signal was suppressed using frequency-selective prepulses. Spectra without water suppression were acquired with identical settings (NSA = 16) and all spectra were fitted with AMARES ${ }^{22}$ in the jMRUI software ${ }^{23}$. The $\mathrm{T}_{2}$-corrected ratios of the $\mathrm{CH}_{2}$ peak, relative to the unsuppressed $\mathrm{T}_{2}$-corrected water resonance was calculated and converted to a tissue fat percentage (weight/weight) by assuming a water content of $71.1 \%$, a $\mathrm{CH}_{2}$-proton density of triglycerides of 60.2 $\mathrm{mol}^{-1}$, a proton density of water of $2 \mathrm{~mol}^{-1}$, a molecular weight of triglycerides and water of $860 \mathrm{~g} / \mathrm{mol}$ and $18 \mathrm{~g} / \mathrm{mol}$, respectively. Three subjects had to be excluded from the $\mathrm{IHL}$ analysis due to poor quality of spectra.

\section{PHOSPHORUS MAGNETIC RESONANCE SPECTROSCOPY}

In a subgroup of eight subjects ATP content was determined at baseline and $4 \mathrm{~h}$ post-exercise in both the fasted and the glucose-supplemented condition. Subjects were positioned in supine position with a $10 \mathrm{~cm}$ diameter transmit/receive surface coil positioned at the level of the liver. MRI scout images were acquired during a breath hold and one-dimensional spectroscopic imaging (SI) was performed with 8 phase-encoding steps and SI slice thickness of $30 \mathrm{~mm}$ ( $T R=4 \mathrm{~s}, \mathrm{n}=512$ points, BW $=4000 \mathrm{~Hz}, \mathrm{NSA}=16$ ). At least one slice was planned to be exclusively in liver tissue. To minimize motion artifacts, subjects were asked to breathe in the rhythm of the measurement and to be at end-expiration during acquisition of spectra. All spectra were fitted with the AMARES algorithm ${ }^{22}$ in the jMRUI software package ${ }^{23}$. The $\gamma^{-}$ ATP resonance was quantified and expressed as ratio of the $\gamma$-ATP to total phosphorus signal in the -25 to $25 \mathrm{ppm}$ frequency region, expressed as ATP/Total $P$ ratio in the current paper. 


\section{STATISTICS}

Data are presented as mean \pm SEM, unless otherwise indicated. Hepatic lipid content was not normally distributed and therefore a non-parametric Friedman test was performed to test if there was an overall effect on IHL. Thereafter a pairwise comparison was performed with a Bonferroni correction for multiple comparisons. A two-way repeated measures ANOVA was performed to compare the mean differences between conditions for ATP, substrate oxidation and plasma values of FA, triglycerides, and glucose. All statistics were performed using SPSS 16.0 (IBM Corporation, Armonk, NY, USA) for Mac and P $<0.05$ was considered statistically significant.

\section{RESULTS}

\section{BASIC CHARACTERISTICS}

Twenty-one middle-aged overweight men participated in this study. The subject characteristics of the entire group are shown in table 1 . The subjects had a wide variety of liver fat content (see figure 2 for basal liver fat content per subject). Of these subjects, eleven subjects met the clinical criteria for NAFLD (> 5.6 \% liver fat $)^{24}$, while in ten subjects hepatic fat content was within the normal physiological range ( $<5.6 \%$ liver fat). There were no significant differences in body weight, BMI, whole body fat percentage and fasting plasma glucose levels between subjects that would qualify as NAFLD and subjects with a normal liver fat content, but the NAFLD subjects were somewhat younger than the subjects with normal liver fat content $(51.7 \pm 5.4$ years vs $58.2 \pm 7.7$ years, $p=0.036)$ and had a higher diastolic blood pressure $(p=0.011)$. As expected, clinical parameters that associate with fatty liver, such as plasma levels of TG and the liver enzymes gamma-GT, ASAT and ALAT were all significantly higher in subjects with a high (> $5.6 \%$ ) liver fat content compared to the subjects with normal liver fat content $(p<0.05)$. However, despite these clinical differences, liver fat content responded similarly in subjects with low and high liver fat content and hence all subjects are treated as one group in the data presented below. 
Table 1. Subject characteristics.

\begin{tabular}{|c|c|c|c|}
\hline & $\mathrm{IHL}<5.6 \%$ & $\mathrm{IHL}>5.6 \%$ & All subjects (pooled) \\
\hline Age (year) & $58.2 \pm 7.7$ & $51.7 \pm 5.4^{*}$ & $54.8 \pm 7.2$ \\
\hline Height (m) & $1.79 \pm 0.05$ & $1.79 \pm 0.03$ & $1.79 \pm 0.04$ \\
\hline Weight (kg) & $92.6 \pm 9.1$ & $97.5 \pm 8.6$ & $95.2 \pm 9.0$ \\
\hline BMI $\left(\mathrm{kg} / \mathrm{m}^{2}\right)$ & $28.7 \pm 1.8$ & $30.4 \pm 2.3$ & $29.6 \pm 2.2$ \\
\hline Fat (\%) & $27.5 \pm 6.2$ & $28.5 \pm 3.1$ & $27.9 \pm 4.9$ \\
\hline $\mathrm{VO}_{2 \max }(\mathrm{ml} / \mathrm{min} / \mathrm{kg})$ & $30.3 \pm 5.7$ & $29.1 \pm 6.1$ & $29.7 \pm 5.8$ \\
\hline $\mathrm{SBP}(\mathrm{mmHg})$ & $135.7 \pm 16.5$ & $144.7 \pm 12.6$ & $140.4 \pm 15.0$ \\
\hline $\mathrm{DBP}(\mathrm{mmHg})$ & $83.3 \pm 12.0$ & $95.1 \pm 6.7^{*}$ & $89.5 \pm 11.1$ \\
\hline Glucose (mmol/l) & $5.3 \pm 0.8$ & $5.6 \pm 0.4$ & $5.4 \pm 0.6$ \\
\hline Gamma-GT (U/I) & $27.6 \pm 9.4$ & $55.5 \pm 26.2^{*}$ & $42.2 \pm 24.2$ \\
\hline ASAT (U/I) & $18.0 \pm 4.5$ & $25.9 \pm 6.6^{*}$ & $22.1 \pm 6.9$ \\
\hline ALAT (U/I) & $25.4 \pm 8.8$ & $44.6 \pm 11.0^{*}$ & $35.5 \pm 13.9$ \\
\hline Triglycerides (mmol/l) & $1.1 \pm 0.3$ & $2.0 \pm 0.5^{*}$ & $1.5 \pm 0.6$ \\
\hline IHL (\%) & $2.7 \pm 1.5$ & $13.1 \pm 6.7^{*}$ & $8.1 \pm 7.2$ \\
\hline
\end{tabular}

${ }^{*} p<0.05$. Data are mean \pm SD.

\section{ENERGY EXPENDITURE AND SUBSTRATE OXIDATION}

No significant differences in energy expenditure between the glucose-fed and the fasted state were found at baseline, during exercise or post-exercise (see table 2). During exercise, there was a significant time $(p<0.001)$ and treatment $(p<0.001)$ effect, without a time*treatment interaction $(p<0.132)$ effect for respiratory quotient (RQ). RQ was significantly higher at every time point, except for baseline, in the glucose-fed state compared with the fasted state $(p<0.01)$ (see figure $3 a$ ), reflecting a higher carbohydrate oxidation in the glucose-fed state compared with the fasted state $(p<0.01)$ and a higher fat oxidation in the fasted state compared with the glucose-fed state (see table 2 for whole body glucose- and fat oxidation). $R Q$ dropped between $t=30$ and $t=120 \mathrm{~min}(p<0.001)$ in both conditions. In the postexercise period, a significant treatment effect $(p<0.001)$ was observed, with RQ remaining significantly higher in the glucose-fed state compared with the fasted state at every time point $(p<0.001)$. Data presented above is acquired from 17 subjects, data from four subjects had to be excluded from the analysis due to missing or poor quality of data. 


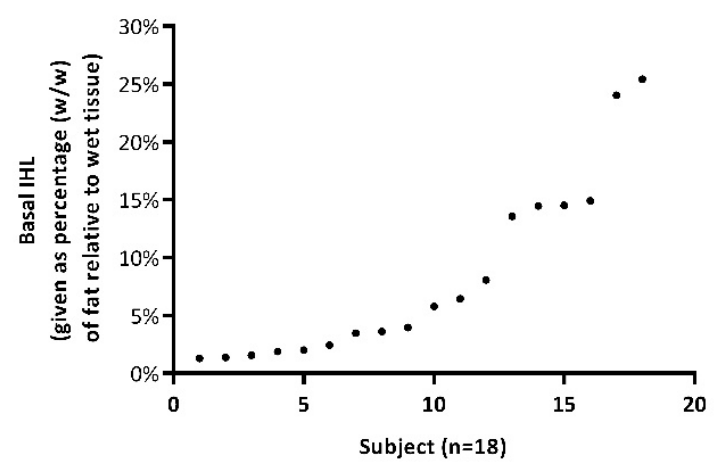

Figure 2. Intrahepatic lipid (IHL) content at baseline for each individual participant given as percentage (weight/weight) of hepatic fat relative to wet tissue, $\mathrm{n}=18$.

\section{PLASMA CONCENTRATIONS}

There was a significant time $(p<0.0001)$, treatment $(p<0.0001)$ and time*treatment interaction $(p<0.0001)$ effect for plasma FA concentrations. Plasma FA concentrations increased with time during exercise and recovery from exercise in the fasted state, with a significant higher plasma FA concentration at the end of exercise $(t=120)$ and $4 h$ post-exercise $(t=360)$ compared with before exercise $(t=-$ $60)(p<0.0001)$, whereas plasma FA concentrations decreased over time in the glucose-fed state $(p=0.002)$. Furthermore, plasma FA concentrations were substantially higher at the end of $2 \mathrm{~h}$ of exercise and $4 \mathrm{~h}$ post-exercise in the fasted state $(p<0.0001)$ compared with the glucose-fed state (figure $3 b)$.

Plasma glucose concentrations showed a significant time $(p<0.0001)$, treatment $(p<0.0001)$ and time*treatment interaction $(p<0.0001)$ effect. Thus, plasma glucose concentrations increased with time during exercise and recovery from exercise in the glucose-supplemented state, with a significant higher plasma glucose concentration at the end of exercise $(t=120)$ and 4 h post-exercise $(t=360)$ compared with before exercise $(t=-60)(p=0.0001)$, whereas plasma glucose concentrations decreased over time in the fasted state $(p=0.0001)$. Moreover, plasma glucose concentrations were higher at the end of $2 \mathrm{~h}$ of exercise and $4 \mathrm{~h}$ post-exercise in the glucose-supplemented state $(p<0.0001)$ compared with the fasted state (figure $3 c)$. Plasma triglyceride concentrations showed a significant time $(p<0.0001)$ and time*treatment interaction $(p<0.0001)$ effect in the fasted condition (figure $3 d$ ). 
Plasma triglyceride concentrations decreased with time from baseline $(\mathrm{t}=-60)$ to post-recovery ( $t=360)$ in the fasted state, whereas it did not change when glucose supplementation was given.

Table 2. Energy expenditure and fat and carbohydrate oxidation during and after exercise in glucose-supplemented and fasted condition.

\begin{tabular}{llccc|ccc}
\hline & & \multicolumn{3}{c}{ Glucose-supplemented } & \multicolumn{3}{c}{ Fasted condition } \\
& Time & E.E. & Fat Ox. & Glucose Ox. & E.E. & Fat Ox. & Glucose Ox. \\
& $\min$ & $\mathrm{kJ} / \mathrm{min}$ & $\mathrm{mg} / \mathrm{min}$ & $\mathrm{mg} / \mathrm{min}$ & $\mathrm{kJ} / \mathrm{min}$ & $\mathrm{mg} / \mathrm{min}$ & $\mathrm{mg} / \mathrm{min}$ \\
\hline Exercise & $\mathbf{3 0}$ & $36 \pm 4$ & $342 \pm 108^{\#}$ & $1442 \pm 273^{\#}$ & $36 \pm 5$ & $457 \pm 139$ & $1125 \pm 337$ \\
& $\mathbf{6 0}$ & $37 \pm 4$ & $512 \pm 133^{\#}$ & $1077 \pm 362^{\#}$ & $37 \pm 5$ & $592 \pm 162$ & $854 \pm 396$ \\
& $\mathbf{9 0}$ & $36 \pm 5$ & $534 \pm 114^{\#}$ & $942 \pm 326^{\#}$ & $36 \pm 5$ & $646 \pm 174$ & $666 \pm 424$ \\
& $\mathbf{1 2 0}$ & $35 \pm 4$ & $548 \pm 135^{\#}$ & $891 \pm 329^{\#}$ & $36 \pm 6$ & $672 \pm 164$ & $600 \pm 438$ \\
\hline Post-exercise & $\mathbf{2 4 0}$ & $5 \pm 1$ & $54 \pm 43^{\#}$ & $231 \pm 107^{\#}$ & $6 \pm 1$ & $107 \pm 21$ & $79 \pm 45$ \\
& $\mathbf{3 0 0}$ & $5 \pm 1$ & $45 \pm 20^{\#}$ & $238 \pm 54^{\#}$ & $5 \pm 1$ & $108 \pm 22$ & $72 \pm 45$ \\
& $\mathbf{3 6 0}$ & $5 \pm 1$ & $45 \pm 18^{\#}$ & $240 \pm 45^{\#}$ & $6 \pm 1$ & $114 \pm 21$ & $70 \pm 45$ \\
\hline
\end{tabular}

${ }^{\#} \mathrm{p}<0.01$ compared with fasted condition. E.E., energy expenditure. Data are mean $\pm \mathrm{SEM}$, $\mathrm{n}=17$.

\section{HEPATIC LIPID CONTENT}

Hepatic lipid content was investigated, by the means of ${ }^{1} \mathrm{H}-\mathrm{MRS}$, before, within $30 \mathrm{~min}$ after cessation of exercise and $4 \mathrm{~h}$ post-exercise. IHL did not change significantly with acute exercise neither in the fasted nor in the glucosesupplemented condition, with IHL after cessation of exercise $(t=120)$ being $8.3 \pm 1.9$ $\%$ of the water resonance in the fasted condition $(p=0.154)$ and $8.4 \pm 1.8 \%$ in the glucose-supplemented condition ( $p=0.181$ ). However, IHL was increased $4 \mathrm{~h}$ postexercise in the fasted condition compared with before exercise $(8.3 \pm 1.8 \%$ of the water resonance before exercise to $8.7 \pm 1.8 \%$ of the water resonance $4 \mathrm{~h}$ postexercise, $\mathrm{p}=0.010)$; this increase in IHL post-exercise was absent in the glucosesupplemented condition (from $8.3 \pm 1.9 \%$ to $8.3 \pm 1.9 \%$ of the water resonance, $\mathrm{p}=0.789$ ) (figure 4a).

When dividing subjects with low $(<5.6 \%)$ and high $(>5.6 \%)$ liver fat content, similar effects are found, with no significant changes in $\mathrm{IHL}$ within $30 \mathrm{~min}$ after cessation of exercise in both conditions and slightly increased $4 \mathrm{~h}$ post-exercise in the fasted 
condition. Furthermore, the increase in IHL 4 h post-exercise was similar for both groups $(p=0.630$ ) (see delta increase in figure $4 b)$.
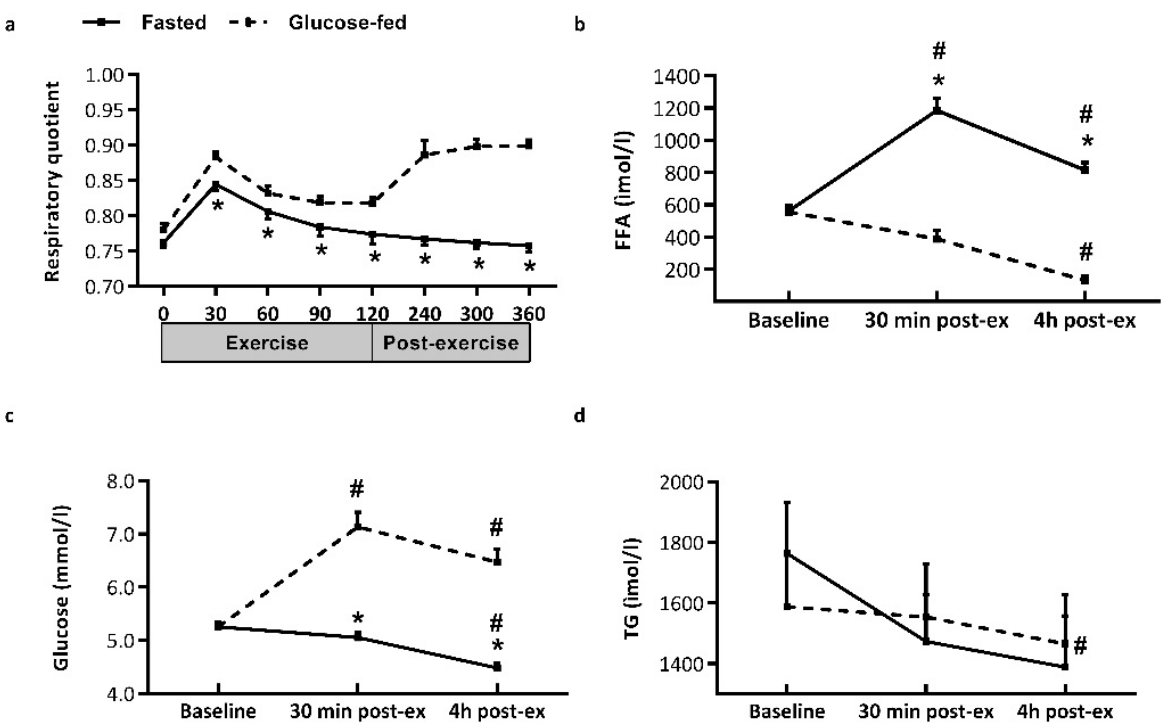

Figure 3. (a) Respiratory quotient during and after $2 \mathrm{~h}$ of cycling at $50 \%$ of maximal power output ( $\left.W_{\max }\right)(n=17)$ and plasma concentrations of (b) free fatty acids (FFA), (c) glucose, (d) and triglycerides (TG), with (open square) and without (filled square) glucose supplementation. ${ }^{*} p<0.05$ compared with glucose-supplemented condition; \#p<0.05 compared with baseline $(\mathrm{t}=-60)$. Data are mean \pm SEM.

\section{HEPATIC ATP/TOTAL P RATIO}

ATP/Total $P$ ratio was not statistically significant different from baseline $4 \mathrm{~h}$ postexercise in neither the fasted $(p=0.086)$ nor the glucose-supplemented $(p=0.582)$ condition (figure $5 a$ ). However, although not statistically significant, we observed a tendency to a decrease in ATP/Total $P$ ratio in the fasted condition. In six out of eight subjects ATP/Total $P$ ratio decreased with $16.9 \pm 3.3 \% 4$ h post-exercise, whereas we did not observe a decrease in ATP/Total $P$ ratio when glucose supplementation was given (See individual data in figure $5 \mathrm{~b}$ and $\mathrm{c}$ ). No correlation was found between IHL and hepatic ATP content in this subgroup of eight subjects. 

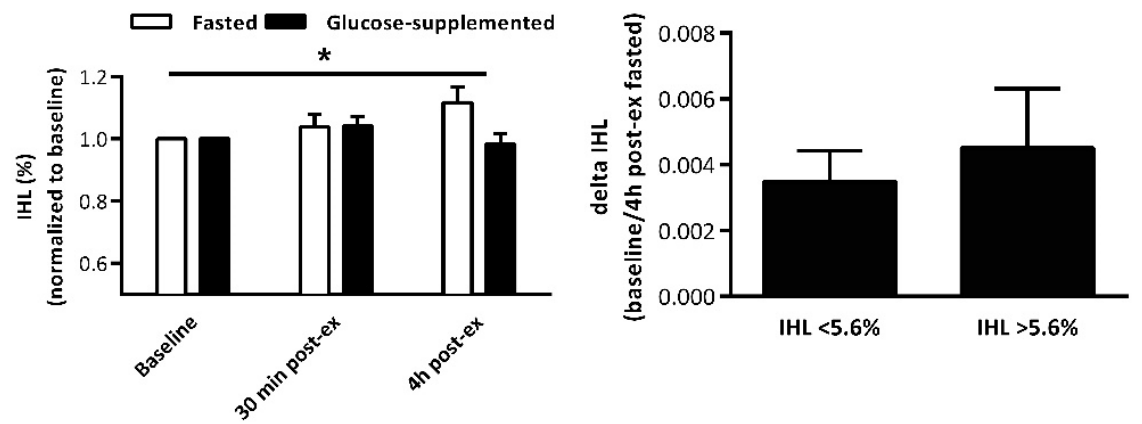

Figure 4. (a) Intrahepatic lipid (IHL) content within 30min after cessation of exercise (30min post-exercise) and $4 \mathrm{~h}$ post-exercise. Because the subjects had a wide variety of liver fat content data was normalized to baseline values, $n=18$, and (b) delta $(\Delta)$ IHL between baseline and $4 \mathrm{~h}$ post-exercise in the fasted condition in subjects with low $(<5.6 \%)$ and high ( $>5.6 \%$ ) liver fat content. $* p<0.05$. Data are mean \pm SEM.

\section{DISCUSSION}

Recent evidence suggests that prolonged exercise training might have a lowering effect on $\mathrm{IHL}^{3-6}$. Here, we examined the effect of an acute bout of exercise on the $\mathrm{IHL}$ in middle-aged overweight men with a wide range of liver fat content and we did not observe a decreased IHL in this population. Moreover, in the recovery phase after exercise, IHL was increased at $4 \mathrm{~h}$ post-exercise in the fasted condition; this increase in IHL was absent when subjects consumed glucose during and after exercise. During and after exercise, circulatory FFA levels are profoundly increased, which may deliver FFA to the liver with increased IHL as a consequence. Of note, glucose supplementation blunts the exercise-induced increase in plasma FFA levels. Therefore, the results of the present study indicate that in spite of strongly stimulated whole body fat oxidation during exercise, an acute bout of exercise is not enough to decrease IHL in this population.

There is an increasing prevalence of obesity worldwide, and obesity is associated with excessive storage of lipids (triglycerides) not only in adipose tissue, but also in non-adipose tissues, such as skeletal muscle and liver. Excessive lipid accumulation in the liver is associated with insulin resistance and type 2 diabetes. Lifestyle 
interventions like exercise training revealed that hepatic fat can be lowered by prolonged exercise training, hence preventing complications associated with hepatic fat accumulation ${ }^{3-6}$, with patients with high liver fat content showing the greatest reduction following physical activity training. Until now there are no studies investigating the acute effects of exercise on hepatic fat accumulation in an overweight middle-aged population at risk of developing fatty liver or with NAFLD already being present. We show here that an acute bout of exercise did not lead to a detectable decrease in $\mathrm{IHL}$, neither in a fasted nor in a glucose-supplemented condition. To our knowledge this is the first study to show this in overweight middle-aged men, which suggests that the long-term effects of physical activity training cannot simply be explained by an additive effect of acute exercise bouts.

Whereas IHL did not significantly change within 30min after cessation of exercise, it was slightly increased $4 \mathrm{~h}$ post-exercise in the fasted condition. This finding is consistent with recent reports in young, healthy subjects, in which one exercise session also lead to an increase in IHL after exercise ${ }^{13,14}$. The authors suggested that increased FFA availability during exercise ${ }^{15,16}$ might be responsible for the increase in IHL. We therefore also examined if exercise would have similar effects on IHL when performed with glucose supplementation during and after exercise. We have previously shown that glucose supplementation during/after exercise markedly blunts the exercise-induced increase in FFA. Consistently, the increase in IHL observed $4 \mathrm{~h}$ post-exercise was absent in the glucose-supplemented condition. Next to reducing FFA levels, glucose supplementation also increases plasma glucose levels and - although not measured - possibly also insulin levels ${ }^{25}$, and both glucose and insulin are able to stimulate de novo lipogenesis ${ }^{26}$, which is an important factor in hepatic fat accumulation ${ }^{27}$. However, since enhanced de novo lipogenesis would result in increased IHL, the absence of an exercise effect on $\mathrm{IHL}$ in the glucose fed condition is most likely due to the reduction in plasma FFA levels, suggesting that indeed FFA availability during and after exercise may be an important factor in determining post-exercise IHL content. However, studies directly measuring FFA fluxes into liver are needed to confirm this hypothesis. 
a

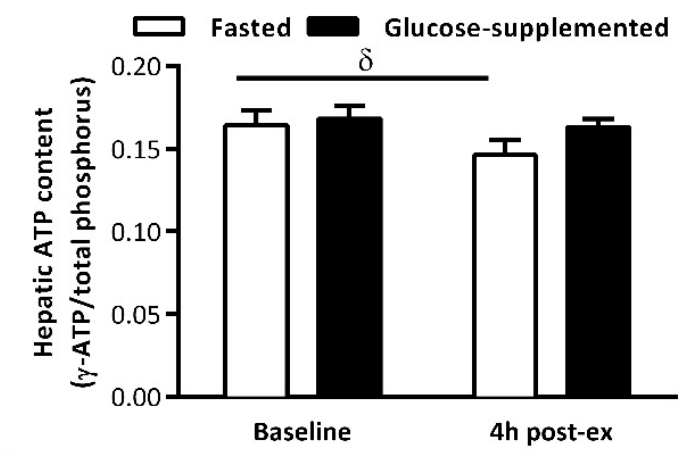

b

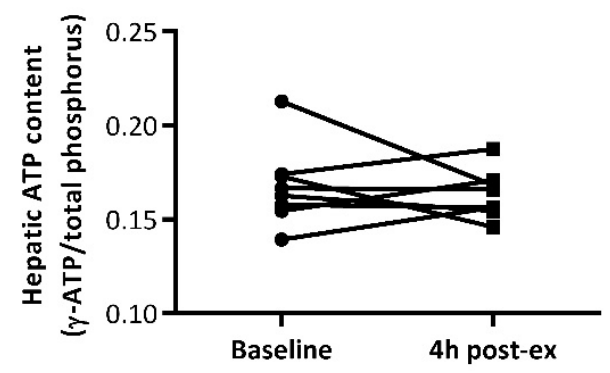

c

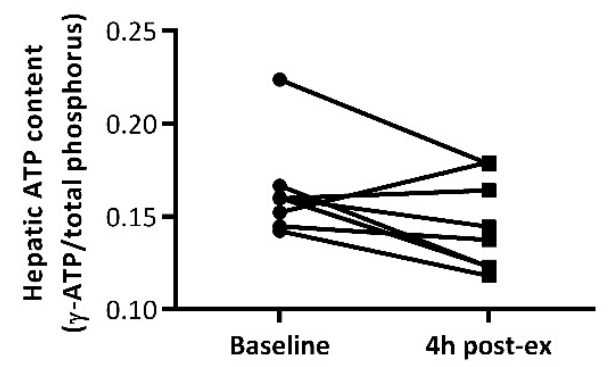

Figure 5. Hepatic ATP/Total P ratio (the relative amount of total phosphorous in the liver) at baseline and $4 \mathrm{~h}$ post-exercise as (a) group average and individual data with (b) and without (c) glucose supplementation. In the fasted condition (without glucosesupplementation) there was a trend to a lower hepatic ATP/Total $P$ ratio $4 \mathrm{~h}$ postexercise compared to baseline. Data are mean \pm SEM.

Next to measuring the IHL content, ATP/Total P ratio was determined at baseline and $4 \mathrm{~h}$ post-exercise in a subgroup of eight subjects. Although, ATP/Total P ratio did not significantly change $4 \mathrm{~h}$ post-exercise in neither the fasted nor the glucosesupplemented condition, ATP/Total $P$ ratio had a tendency to be decreased in six out of eight subjects $4 \mathrm{~h}$ post-exercise in the fasted condition. It has been suggested that hepatic ATP levels are decreased in conditions such as insulin resistance and type 2 diabetes mellitus ${ }^{18,28}$, and that hepatocellular ATP levels are negatively correlating with liver fat in human subjects, suggesting lipotoxicity. In agreement 
with these data the current study reports a trend to decreased ATP levels along with increased IHL, although no correlation between hepatic lipid content and hepatic ATP levels was found. Interestingly, a recent study investigating the energy charge in the liver of mice after one single bout of exercise found a clear increase of AMP and a strong decrease of ATP in the liver ${ }^{29}$, suggesting that the reduction in ATP levels after exercise is a direct consequence of exercise. In the current study we did observe a non-significant decrease in ATP/Total $P$ ratio after exercise in the fasted condition, whereas no decrease in ATP/Total $\mathrm{P}$ ratio was observed after exercise in the glucose-supplemented condition. This suggests that the elevation of IHL upon exercise instead of exercise per se - may be responsible for the reduced hepatic energy status of the liver. However, investigation of larger subject groups will have to determine to what extent the decrease in ATP/Total P ratio is related to increase in IHL.

In summary, we did not observe a decrease in liver fat after acute exercise in overweight middle-aged men. Moreover, IHL was increased post-exercise in the fasted state, an effect that is most likely due to the exercise-induced increase in plasma FFA levels. These data suggest that acute exercise is not responsible for the exercise-lowering effects on liver fat, and that a single bout of exercise may not be able to lower IHL, not even in a population with elevated IHL. 


\section{REFERENCES}

1. Dela, F. Other adaptations to training/inactivity in type 2 diabetics and other groups with insulin resistance: emphasis on prevention of CHD. Applied physiology, nutrition, and metabolism = Physiologie appliquee, nutrition et metabolisme 32, 602-606 (2007).

2. Gordon, B.A., Benson, A.C., Bird, S.R. \& Fraser, S.F. Resistance training improves metabolic health in type 2 diabetes: a systematic review. Diabetes research and clinical practice 83, 157-175 (2009).

3. Sullivan, S., Kirk, E.P., Mittendorfer, B., Patterson, B.W. \& Klein, S. Randomized trial of exercise effect on intrahepatic triglyceride content and lipid kinetics in nonalcoholic fatty liver disease. Hepatology 55, 1738-1745 (2012).

4. Hallsworth, K., et al. Resistance exercise reduces liver fat and its mediators in non-alcoholic fatty liver disease independent of weight loss. Gut 60, 1278-1283 (2011).

5. Finucane, F.M., et al. The effects of aerobic exercise on metabolic risk, insulin sensitivity and intrahepatic lipid in healthy older people from the Hertfordshire Cohort Study: a randomised controlled trial. Diabetologia 53, 624-631 (2010).

6. Chen, S.M., et al. Effects of therapeutic lifestyle program on ultrasounddiagnosed nonalcoholic fatty liver disease. J Chin Med Assoc 71, 551-558 (2008).

7. Magkos, F. Putative factors that may modulate the effect of exercise on liver fat: insights from animal studies. Journal of nutrition and metabolism 2012, 827417 (2012).

8. Magkos, F. Exercise and fat accumulation in the human liver. Curr Opin Lipidol 21, 507-517 (2010).

9. Rogers, M.A. Acute effects of exercise on glucose tolerance in non-insulindependent diabetes. Medicine and science in sports and exercise 21, 362368 (1989).

10. Frank, P., Katz, A., Andersson, E. \& Sahlin, K. Acute exercise reverses starvation-mediated insulin resistance in humans. American journal of physiology 304, E436-443 (2013). 
11. Mackenzie, R., et al. Intermittent exercise with and without hypoxia improves insulin sensitivity in individuals with type 2 diabetes. The Journal of clinical endocrinology and metabolism 97, E546-555 (2012).

12. Johnson, N.A., et al. Effect of prolonged exercise and pre-exercise dietary manipulation on hepatic triglycerides in trained men. European journal of applied physiology 112, 1817-1825 (2011).

13. Bucher, J., et al. The effect of a single $2 \mathrm{~h}$ bout of aerobic exercise on ectopic lipids in skeletal muscle, liver and the myocardium. Diabetologia (2014).

14. Egger, A., et al. The effect of aerobic exercise on intrahepatocellular and intramyocellular lipids in healthy subjects. PLoS One 8, e70865 (2013).

15. Schrauwen, P., et al. Effect of acute exercise on uncoupling protein 3 is a fat metabolism-mediated effect. American journal of physiology 282, E1117 (2002).

16. Bilet, L., et al. Exercise-induced modulation of cardiac lipid content in healthy lean young men. Basic research in cardiology 106, 307-315 (2010).

17. Donnelly, K.L., et al. Sources of fatty acids stored in liver and secreted via lipoproteins in patients with nonalcoholic fatty liver disease. J Clin Invest 115, 1343-1351 (2005).

18. Szendroedi, J., et al. Abnormal hepatic energy homeostasis in type 2 diabetes. Hepatology 50, 1079-1086 (2009).

19. Kuipers, H., Verstappen, F.T., Keizer, H.A., Geurten, P. \& van Kranenburg, $G$. Variability of aerobic performance in the laboratory and its physiologic correlates. International journal of sports medicine 6, 197-201 (1985).

20. Siri, W.E. The gross composition of the body. Advances in biological and medical physics 4, 239-280 (1956).

21. van Herpen, N.A., Schrauwen-Hinderling, V.B., Schaart, G., Mensink, R.P. \& Schrauwen, P. Three weeks on a high-fat diet increases intrahepatic lipid accumulation and decreases metabolic flexibility in healthy overweight men. The Journal of clinical endocrinology and metabolism 96, E691-695 (2011).

22. Vanhamme, L., van den Boogaart, A. \& Van Huffel, S. Improved method for accurate and efficient quantification of MRS data with use of prior knowledge. J Magn Reson 129, 35-43 (1997).

23. Naressi, A., et al. Java-based graphical user interface for the MRUI quantitation package. Magma (New York, N.Y 12, 141-152 (2001). 
24. Szczepaniak, L.S., et al. Magnetic resonance spectroscopy to measure hepatic triglyceride content: prevalence of hepatic steatosis in the general population. American journal of physiology 288, E462-468 (2005).

25. Stellingwerff, T., et al. Carbohydrate supplementation during prolonged cycling exercise spares muscle glycogen but does not affect intramyocellular lipid use. Pflugers Archiv : European journal of physiology 454, 635-647 (2007).

26. Hellerstein, M.K. De novo lipogenesis in humans: metabolic and regulatory aspects. European journal of clinical nutrition 53 Suppl 1, S53-65 (1999).

27. Ferre, P. \& Foufelle, F. Hepatic steatosis: a role for de novo lipogenesis and the transcription factor SREBP-1c. Diabetes, obesity \& metabolism 12 Suppl 2, 83-92 (2010).

28. Schmid, A.l., et al. Liver ATP synthesis is lower and relates to insulin sensitivity in patients with type 2 diabetes. Diabetes Care 34, 448-453 (2011).

29. Camacho, R.C., Donahue, E.P., James, F.D., Berglund, E.D. \& Wasserman, D.H. Energy state of the liver during short-term and exhaustive exercise in C57BL/6J mice. American journal of physiology 290, E405-408 (2006). 



\section{CHAPTER 5}

EXERCISE TRAINING REDUCES LIVER FAT CONTENT AND IMPROVES PERIPHERAL BUT NOT HEPATIC INSULIN SENSITIVITY IN OVERWEIGHT AND OBESE MEN WITH NONALCOHOLIC FATTY LIVER

Bram Brouwers, Vera B. Schrauwen-Hinderling, Anne Gemmink, Bas Havekes, Yvonne Bruls, Dennis Dahlmans, Matthijs K.C. Hesselink, Patrick Schrauwen

An adjusted version has been submitted 


\section{ABSTRACT}

Elevated hepatic lipid (intrahepatic lipid, IHL) content is associated with hepatic insulin resistance. We examined if exercise training decreased IHL content in BMImatched overweight/obese control subjects (CTRL), non-alcoholic fatty liver (NAFL) subjects and type 2 diabetes mellitus (T2DM) patients. Furthermore, we tested whether exercise training induced changes in $\mathrm{IHL}$ content coincided with improvements in hepatic insulin sensitivity.

Eleven sedentary overweight/obese male CTRL subjects, 11 male NAFL subjects and 13 male T2DM patients of comparable body weight, BMI and aerobic capacity $\left(\mathrm{VO}_{2 \max }\right)$ participated in a well-controlled 12 week progressive, combined endurance and resistance training program. IHL content (proton magnetic resonance spectroscopy $\left.\left({ }^{1} \mathrm{H}-\mathrm{MRS}\right)\right)$, insulin sensitivity (2-step hyperinsulinemiceuglycemic clamp) and substrate oxidation (indirect calorimetry) were measured before and after exercise training.

All 3 groups significantly improved $\mathrm{VO}_{2 \max }(p<0.001), W_{\max }(p<0.001)$ and $1 R M$ $(p<0.001)$ upon exercise training. The increase in $\mathrm{VO}_{2 \max }$ was least prominent in T2DM patients $\left(\mathrm{VO}_{2 \max }\right.$ from $25.6 \pm 1.0$ to $\left.26.8 \pm 0.9 \mathrm{ml} / \mathrm{min} / \mathrm{kg}\right)$, whereas increases in CTRL and NAFL subjects were more prominent $\left(\mathrm{VO}_{2 \max }\right.$ from $27.6 \pm 1.4$ to $30.4 \pm$ 1.5 and $26.1 \pm 1.4$ to $29.9 \pm 1.4 \mathrm{ml} / \mathrm{min} / \mathrm{kg}$ in CTRL and NAFL, respectively). IHL content significantly decreased by $22.7 \%$ in NAFL subjects $(p=0.038)$. No significant alteration in IHL content was found in CTRL subjects (from $1.9 \pm 0.4$ to $1.5 \pm 0.3 \%$, $\mathrm{p}=0.182$ ) and T2DM patients (from $8.9 \pm 3.3$ to $9.9 \pm 4.1 \%, p=0.378$ ). Glucose disposal $\left(R_{d}\right)$ significantly improved in NAFL (delta $R_{d}+39.0 \%, p=0.012$ ) and CTRL (delta $R_{d}+22.9 \%, p=0.031$ ) subjects. Interestingly though, insulin-stimulated suppression of endogenous glucose production (delta EGP \%) and insulinstimulated suppression of free fatty acids (delta FFA \%) were unaffected after exercise training. Surprisingly, T2DM patients did not significantly improve delta $R_{d}$, delta EGP \% or delta FFA \% upon exercise training.

Thus, exercise training resulted in a significant drop in IHL content in NAFL subjects. Next to IHL content, exercise training improved skeletal muscle insulin sensitivity in NAFL and CTRL subjects, without affecting hepatic or adipose tissue insulin sensitivity. Surprisingly, exercise training had no effect on IHL content and insulin sensitivity in T2DM patients. 


\section{INTRODUCTION}

The prevalence of obesity and obesity-related diseases has increased dramatically over the past decades ${ }^{1}$. Non-alcoholic fatty liver (NAFL) is a condition characterized by excessive fat accumulation in the liver. NAFL is affecting up to $30 \%$ of the general population $^{2}$ and its prevalence increases to $50-70 \%$ in obesity ${ }^{3}$. High liver fat (intrahepatic lipid, IHL) content is associated with impaired insulin sensitivity, and people with NAFL are characterized by hepatic, skeletal muscle and adipose tissue insulin resistance ${ }^{4-6}$. As a result, NAFL has been linked to the development of type 2 diabetes mellitus (T2DM) and the incidence of NAFL in T2DM is predicted to be as high as $70 \%{ }^{7}$.

High-caloric intake and lack of physical activity are two main factors responsible for the increased prevalence of NAFL. Therefore lifestyle interventions are considered a cornerstone for treatment ${ }^{8}$. Several dietary intervention studies have shown that a reduction in body weight coincides with a significantly decreased IHL content ${ }^{9-12}$. However, weight loss is difficult to achieve and maintain. Interestingly, addition of physical activity to a diet intervention promotes a greater reduction in hepatic fat content, independent of changes in body weight ${ }^{12}$.

Current American Heart Association and American Diabetes Association guidelines target $150 \mathrm{~min}$ of moderate-intensity or $60 \mathrm{~min}$ of vigorous physical activity per week to prevent and treat obesity-related diseases ${ }^{13}$. We and others have previously shown that exercise training is an effective way to improve hepatic and skeletal muscle insulin sensitivity in insulin resistant and T2DM subjects ${ }^{14-16}$. Interestingly, cross-sectional studies have shown that higher levels of physical activity are also associated with lower levels of $\mathrm{IHL}$ content ${ }^{17,18}$. Furthermore, recent intervention studies concluded that both aerobic ${ }^{8,19-21}$ and resistance ${ }^{8,22}$ training were effective in reducing IHL content in NAFL subjects. Only two of those studies measured insulin sensitivity (using HOMA-IR). No improvement in HOMAIR was observed after aerobic exercise training for 4 weeks ${ }^{20}$, while 8 weeks of resistance exercise training did improve HOMA-IR significantly ${ }^{22}$. In healthy overweight and obese subjects one study reported beneficial effects of exercise training on $\mathrm{IHL}$ content ${ }^{23}$, whereas others reported no effects of exercise training 
on hepatic lipid content ${ }^{24,25}$ in the presence ${ }^{24}$ or absence ${ }^{25}$ of improvements in insulin sensitivity.

Thus, consensus is lacking on whether exercise training can decrease hepatic fat content in all individuals and whether a putative decrease in liver fat coincides with improvements in hepatic insulin sensitivity. The aim of our study was therefore to examine if a well-controlled 12 week progressive, combined endurance and resistance training program in BMI-matched overweight/obese control (CTRL) subjects, NAFL subjects and T2DM patients results in reduced IHL content, and whether this drop coincides with improved hepatic insulin sensitivity.

\section{RESEARCH DESIGN AND METHODS}

Eleven overweight/obese male CTRL subjects, 11 male NAFL subjects and 13 male T2DM patients matched for BMI were included in the study. Subjects were sedentary, middle-aged ( $40-70$ years old) with stable dietary habits. General exclusion criteria were unstable body weight, cardiac disease, impaired renal function, hemoglobin $<7.5 \mathrm{mmol} / \mathrm{l}$, use of beta-blockers, use of anti-thrombotic medication, elevated blood pressure (>160/100 mmHg), claustrophobia, contraindications for MRI, recent participation in a weight loss or vigorous exercise program, history of substantial alcohol use ( $>3$ units per day), history of drug abuse, use of insulin therapy and use of medication known to interfere with glucose homeostasis (except for antidiabetic medication in T2DM patients). CTRL subjects were selected on having a liver fat content $\leq 4 \%$ as measured with ${ }^{1} \mathrm{H}-\mathrm{MRS}$, in the absence of liver disease or liver dysfunction (defined as alanine aminotransferase (ALAT) $>2.5 \times$ normal values) and had to be normoglycemic according to the WHO criteria. NAFL subjects were selected on having a liver fat content $\geq 5 \%$ as measured with ${ }^{1} \mathrm{H}-\mathrm{MRS}$, with a fasting plasma glucose (FPG) concentration $<7.0 \mathrm{mmol} / \mathrm{l}$. T2DM patients were allowed to be on sulphonyl urea or metformin therapy for at least 6 months with constant dose for at least 2 months or on a dietary treatment for at least 6 months, with FPG concentration $\geq 7.0 \mathrm{mmol} / \mathrm{l}$ and $<10.0 \mathrm{mmol} / \mathrm{l}$ at the time of screening. T2DM patients were not screened for liver fat content. 
Subjects were recruited via advertisements in local newspapers. Before the onset of the study, routine medical laboratory testing, a medical history and physical examination was performed, and a resting electrocardiogram was taken. Maximal work load $\left(\mathrm{W}_{\max }\right)$ and maximal aerobic capacity $\left(\mathrm{VO}_{2 \max }\right)$ were assessed during a graded cycling test with concurrent ECG until exhaustion ${ }^{26}$. Body composition was determined by DXA (Hologic Discovery A, Waltham, MA, USA).

All subjects gave written informed consent before participation in the study. The Maastricht University Medical Ethical Committee approved the study. The study was performed according to the principles expressed in the most recent version of the declaration of Helsinki.

\section{EXERCISE TRAINING PROTOCOL}

Subjects were enrolled in a tightly controlled, progressive, combined endurance and resistance training program for 12 weeks. Aerobic exercise was carried out on a cycling ergometer twice a week for $30 \mathrm{~min}$ at $70 \%$ of the previously determined $\mathrm{W}_{\text {max. }}$. Resistance exercise was performed once a week and comprised three series of ten repetitions at $60 \%$ of subjects' previously determined one repeated maximum (1RM) and focused on large muscle groups (chest press, lat pull down, leg extension, shoulder press, horizontal row, leg press, triceps curls and biceps curls). The $1 \mathrm{RM}$ test was preceded by a familiarization trial. Warming-up and cooling-down sessions of $5 \mathrm{~min}$ were performed on a stationary bike at $45 \%$ of $\mathrm{W}_{\text {max }}$. Every 4 weeks, $1 \mathrm{RM}$ and $\mathrm{VO}_{2 \max }$ were reassessed, and training loads were readjusted accordingly. Supervised training sessions were performed with 3-4 subjects at a time.

\section{PROTON MAGNETIC RESONANCE SPECTROSCOPY}

IHL content was measured in the morning $(6.00-7.00$ A.M.) of the 2-step hyperinsulinemic-euglycemic clamp test day. Proton magnetic resonance spectroscopy $\left({ }^{1} \mathrm{H}-\mathrm{MRS}\right)$ was used to quantify $\mathrm{IHL}$ content as described earlier ${ }^{27}$, however using a five element coil on a $3 \mathrm{~T}$ whole body scanner (Philips Achieva 3Tx; 
Philips Healthcare, Best, The Netherlands) and a repetition time $=4000 \mathrm{msec}$, echo time $=32.5 \mathrm{msec}$, and number of averages $=64$. To minimize motion artifacts, subjects were asked to breathe in the rhythm of the measurement and to be at end-expiration during acquisition of spectra. To determine the intensity of the lipid peak, the water signal was suppressed using frequency-selective prepulses. The unsuppressed water resonance was used as internal reference (number of averages = 32) and spectra were fitted with MATLAB R2014b (Mathworks, Natick, MA, USA). Values are given as $\mathrm{T2}$-corrected ratios of the $\mathrm{CH}_{2}$ resonance, relative to the unsuppressed water resonance, as percentage.

\section{PHOSPHORUS MAGNETIC RESONANCE SPECTROSCOPY}

Measurements were performed on a 3 T whole body scanner (Philips Achieva 3Tx; Philips Healthcare, Best, The Netherlands). Subjects were positioned in a supine position. A $14 \mathrm{~cm}$ diameter circular ${ }^{31} \mathrm{P}$ receive and transmit surface coil was positioned at the level of the liver and secured in place using Velcro bands. Before spectroscopic acquisition, fifteen axial, coronal and sagittal ${ }^{1} \mathrm{H}-\mathrm{MR}$ images (slice thickness $=10 \mathrm{~mm}$, field of view $=450 * 450 \mathrm{~mm}$, matrix size $=192 * 96, \mathrm{TE}=1.82$ $\mathrm{msec}, \mathrm{TR}=3.8 \mathrm{msec}$ ) from the liver region were acquired using a gradient-echo sequence during one breath hold. The VOI was positioned parallel to the coil at a distance of $5 \mathrm{~cm}$ from the center of the coil. Manual tuning and matching of the ${ }^{31} \mathrm{P}$ receive and transmit surface coil was performed and pencil bean shimming was performed. Localized phosphorus spectra were obtained using Image Selected In Vivo Spectroscopy (ISIS). Sequence parameters were repetition time (TR): 5500 msec, number of single acquisitions (NSA): 128, number of sample points: 1024 and spectral bandwidth: $3000 \mathrm{~Hz}$. The carrier frequency was chosen to match the resonance frequency of $\mathrm{y}$-ATP.

\section{HYPERINSULINEMIC-EUGLYCEMIC CLAMP}

All participants underwent a 2-step $6 \mathrm{~h}$ hyperinsulinemic-euglycemic clamp (10 and $\left.40 \mathrm{mU} / \mathrm{m}^{2} / \mathrm{min}\right)^{28}, 4$ days prior to the start of the exercise training protocol and $60 \mathrm{~h}$ after the last exercise bout. After an overnight fast, participants received a primed 
continuous infusion of $\left[6,6-{ }^{2} \mathrm{H}_{2}\right]$ glucose $(0.04 \mathrm{mg} / \mathrm{kg} / \mathrm{min})$ to determine rates of endogenous glucose production (EGP) and whole body glucose disposal rates $\left(R_{d}\right)$ as previously described ${ }^{29}$. After $180 \mathrm{~min}$, low-dose insulin infusion was started (10 $\mathrm{mU} / \mathrm{m}^{2} / \mathrm{min}$ ) for $3.5 \mathrm{~h}$ until a steady state was reached, after which blood sampling and indirect calorimetry was performed during $30 \mathrm{~min}$. Thereafter, high-dose insulin infusion was started $\left(40 \mathrm{mU} / \mathrm{m}^{2} / \mathrm{min}\right)$ for $1.5 \mathrm{~h}$ until steady state was reached, after which blood sampling and indirect calorimetry were repeated for $30 \mathrm{~min}$. During the clamp, oxygen consumption and carbon dioxide production were measured with daily calibrated automated respiratory gas analyzer using a ventilated hood system (Omnical, IDEE, Maastricht, The Netherlands). Whole body glucose and fat oxidation rates were calculated using stoichiometric equations based on measured oxygen consumption and carbon dioxide concentrations ${ }^{30}$ with the assumption that protein oxidation was negligible.

\section{TRACER CALCULATIONS}

Isotopic enrichment of plasma glucose was determined by electric impact ionization gas chromatography-mass spectroscopy as previously described ${ }^{31}$. Steele's single-pool steady state equations were used to calculate glucose rate of appearance $\left(R_{a}\right)$ and $R_{d}{ }^{32}$. Volume of distribution was assumed to be $0.160 \mathrm{l} / \mathrm{kg}$ for glucose. Insulin-stimulated glucose disposal (delta $R_{d}$ ) was computed as the difference between $R_{d}$ under insulin-stimulated conditions and $R_{d}$ under basal and non-insulin-stimulated conditions. Endogenous glucose production (EGP) was calculated as $R_{a}$ - exogenous glucose infusion rate. Non-oxidative glucose disposal (NOGD) was calculated as $R_{d}$ - glucose oxidation.

\section{BLOOD SAMPLE ANALYSIS}

On the 2-step hyperinsulinemic-euglycemic clamp test day, arterialized blood samples were collected and immediately centrifuged at high speed. Plasma was frozen in liquid nitrogen and stored at $-80^{\circ} \mathrm{C}$ until assayed. Plasma free fatty acids and FPG were measured with enzymatic assays automated on a Cobas Fara/Mira (FFA: Wako Nefa C test kit; Wako Chemicals, Neuss, Germany) (FPG: hexokinase 
method; La Roche, Basel, Switzerland). Plasma triglycerides (TG) were measured colorimetrically (Roche, Vienna, Austria). Insulin and serum liver function parameters (alanine transaminase (ALAT), aspartate aminotransferase (ASAT), $\gamma$ glutamyl transpeptidase (GGT)) were routinely measured during the screening visit and analyzed at the clinical chemistry department.

\section{MUSCLE BIOPSY AND ANALYSIS}

Muscle biopsies were taken from the musculus vastus lateralis, prior to the 2-step hyperinsulinemic-euglycemic clamp under local anesthesia (2 \% xylocaine), according to the Bergstrom technique ${ }^{33}$. Any visible non-muscle material was dissected from muscle tissue. Samples were stored at $-80^{\circ} \mathrm{C}$ until further use.

Oxidative phosphorylation (OXPHOS) was measured in whole muscle by western blotting as a reflection of mitochondrial density, as previously described ${ }^{14}$. OXPHOS proteins were detected using a monoclonal antibody cocktail of 5 monoclonal antibodies directed against the different OXPHOS complexes (MS601, Mitosciences, Eugene, OR, USA). Gels were loaded with equal amounts of protein of pre- and post-exercise training lysates to allow valid comparison between preand post-exercise training samples. To adjust for inter-gel variation, the optical density of the band of interest per subject was normalized to the mean optical density of the complete gel. Protein content is expressed as arbitrary units (AU).

\section{STATISTICS}

All values are reported as the mean \pm standard error of the mean (SEM). Subject baseline characteristics were analyzed using one-way ANOVA with Tukey or GamesHowell post-hoc correction. Exercise training effects were analyzed using a twoway ANOVA model for repeated measures using CTRL, NAFL and T2DM subjects as between-subject variables and pre- and post-training data as repeated withinsubject variables. Statistical significance was set at $P \leq 0.05$. Normality was assessed by the Shapiro-Wilk test or determination of skewness and kurtosis. Homogeneity 
of variances was assessed by Levene's test for equality of variances. All statistic calculations were performed using IBM SPSS 21 (SPSS, Chicago, IL, USA).

\section{RESULTS}

\section{SUBJECT BASELINE CHARACTERISTICS}

Groups were carefully matched for BMI (table 1). NAFL subjects tended to be younger than any of the other groups $(57.6 \pm 2.5,54.5 \pm 2.0$ and $61.5 \pm 1.6$ years, ANOVA $p=0.055$ for CTRL, NAFL and T2DM, respectively). Body composition and physical fitness were similar across groups at the onset of the study (table 1). By design, FPG levels after an overnight fast were significantly different across groups (5.4 $\pm 0.2,5.6 \pm 0.2$ and $8.7 \pm 0.6 \mathrm{mmol} / \mathrm{l}, \mathrm{p}<0.001$ for CTRL, NAFL and T2DM, respectively), and post-hoc analysis confirmed that FPG was significantly higher in the T2DM patients compared to the CTRL $(p<0.001)$ and NAFL $(p<0.001)$ subjects. Also fasting insulin levels were significantly different across groups $(9.2 \pm 0.7,15.2$ \pm 1.9 and $14.9 \pm 2.6 \mathrm{mU} / \mathrm{l}, \mathrm{p}=0.012$ for CTRL, NAFL and T2DM, respectively). Posthoc analysis revealed significantly higher fasting insulin levels in NAFL than in CTRL subjects $(p=0.030)$. Plasma glucose values $120 \mathrm{~min}$ after an oral glucose tolerance test (OGTT) bolus were significantly higher in NAFL $(6.7 \pm 0.7 \mathrm{mmol} / \mathrm{l})$ compared to CTRL $(4.8 \pm 0.3 \mathrm{mmol} / \mathrm{I})(\mathrm{p}=0.021)$. For T2DM patients, no OGTT was performed. Plasma TG and free glycerol levels were similar across groups at the onset of the study. Subject characteristics are summarized in table 1.

\section{EXERCISE CAPACITY}

Subjects participated in a supervised exercise training protocol. Compliance was comparable between groups, with average attendance $96.0 \pm 1.5,94.7 \pm 1.5$ and $96.5 \pm 1.2 \%$ in CTRL, NAFL and T2DM, respectively ( $p=0.798)$.

To test if the exercise training intervention was successful, we first examined if exercise training increased maximal aerobic capacity. 


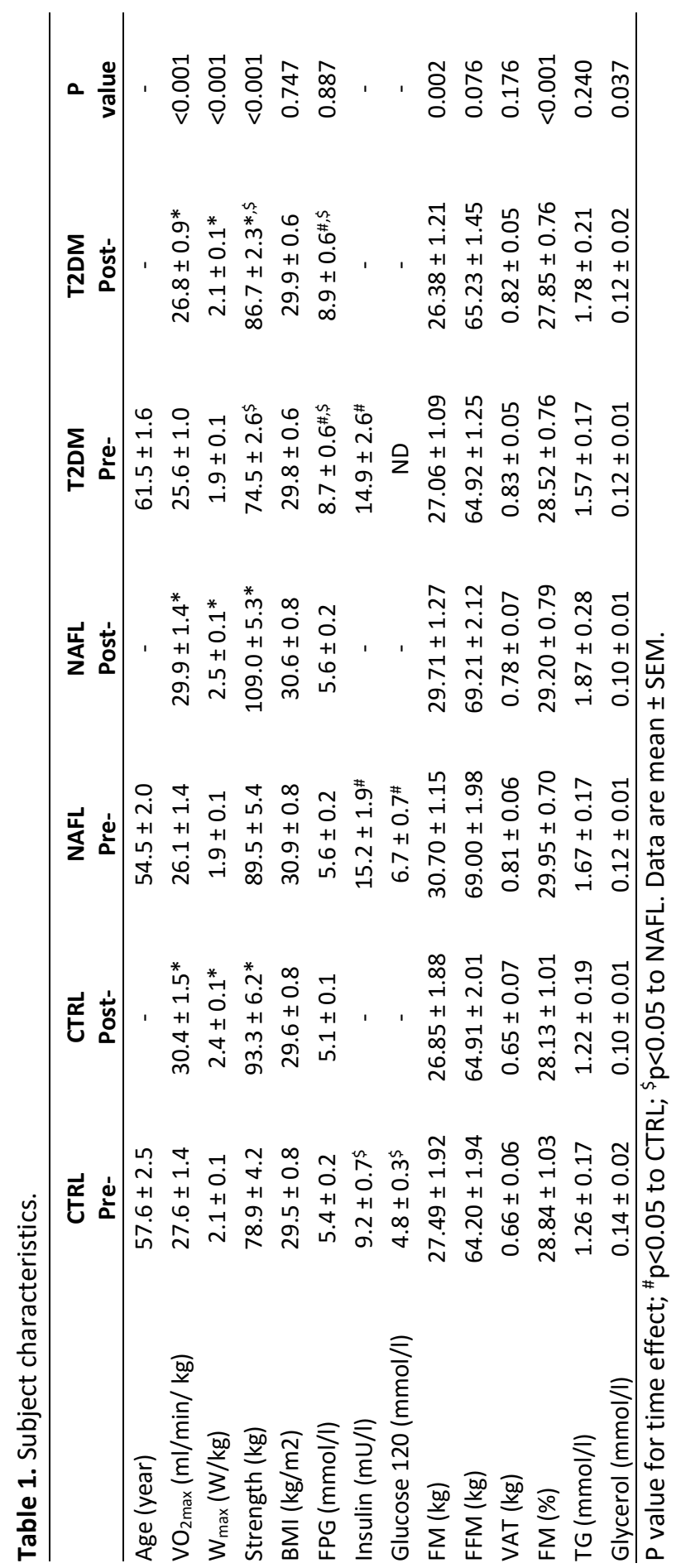


Indeed, there was a significant time $(p<0.001)$ and time* group $(p=0.012)$ effect in $\mathrm{VO}_{2 \max }$ upon exercise training (table 1). Although $\mathrm{VO}_{2 \max }$ increased significantly in all three groups $(p<0.001)$, the increase in $\mathrm{VO}_{2 \max }$ was significantly larger in the NAFL subjects when compared to T2DM patients $(p=0.009)$. Also $W_{\max }$ showed a significant time $(p<0.001)$ and time*group $(p=0.001)$ effect upon exercise training. Again, $W_{\max }$ increased significantly in all three groups (table $1, p<0.001$ ), but consistent with the observation in $\mathrm{VO}_{2 \max }$, the increase in $\mathrm{W}_{\max }$ was significantly larger in the NAFL subjects when compared to CTRL subjects $(p=0.028)$ and T2DM patients $(p=0.001)$.

We also investigated if exercise training improved muscle strength. Indeed, there was a significant time $(p<0.001)$ and group $(p=0.011)$ effect for $1 R M$. All 3 groups improved $1 \mathrm{RM}$ to a similar extend by on average $18.9 \%$. Both before and after exercise training, NAFL subjects were significantly stronger than T2DM patients (table 1, $\mathrm{p}=0.009$ ).

\section{BODY COMPOSITION}

We next examined if exercise training had an effect on body composition. Exercise training induced no significant time, group or time* group effect on BMI or visceral adipose tissue mass (table 1$)$. There was a time effect for total fat mass $(p=0.002)$ and fat percentage $(p<0.001)$ (table 1$)$, with a small but significant decrease in fat mass and fat percentage after exercise training. No group or time*group effect could be observed for neither, fat mass or fat percentage. Fat free mass did not significantly change after exercise training, although there was a trend for a small increase over time (table 1, $\mathrm{p}=0.076$ ).

\section{INTRAHEPATIC LIPID CONTENT AND HEPATIC ENERGY STATUS}

The main objective of the current study was to examine if exercise training affects $\mathrm{IHL}$ content. Interestingly, we found that IHL content showed a time*group $(p=0.050)$, but no significant time $(p=0.329)$ or group $(p=0.089)$ effect upon exercise training. Thus, IHL content decreased significantly by approximately $22.7 \%$ in NAFL subjects (from $9.7 \pm 2.0$ to $7.5 \pm 1.3 \%, p=0.038$ ). IHL content did not significantly 
change in either CTRL subjects (from $1.9 \pm 0.4$ to $1.5 \pm 0.3 \%, p=0.182$ ) or T2DM patients (from $8.9 \pm 3.3$ to $9.9 \pm 4.1 \%, p=0.378$ ) (figure 1 ).

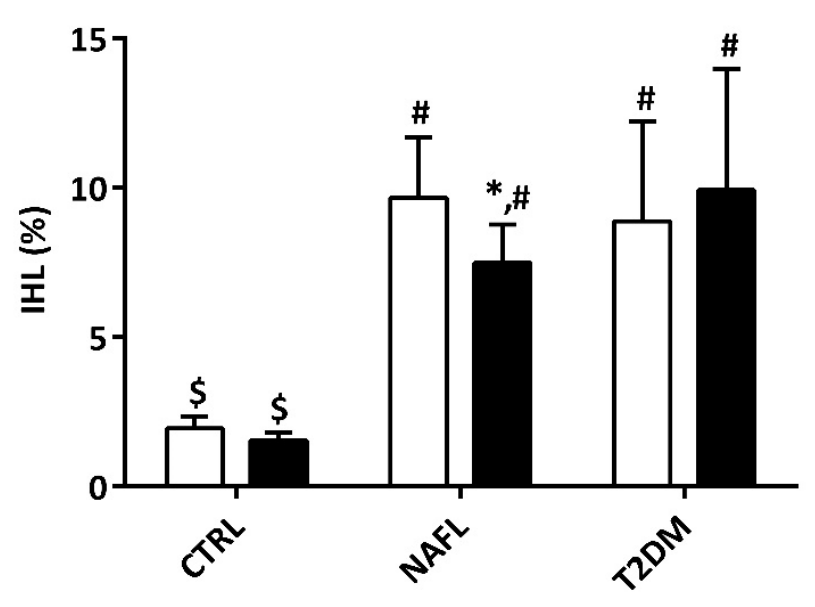

Figure 1. Intrahepatic lipid (IHL) content pre- (white columns) and post- (black columns) exercise training. $\mathrm{IHL}$ is expressed as the relative $\mathrm{CH}_{2}$ peak intensity, compared to the unsuppressed $\mathrm{H}_{2} \mathrm{O}$ peak, corrected for $\mathrm{T} 2$ relaxation. $\mathrm{n}=11$ for $\mathrm{CTRL}, \mathrm{n}=11$ for NAFL, $n=13$ for T2DM. $* p<0.05$ from baseline; ${ }^{\#} p<0.05$ to CTRL; ${ }^{\$} p<0.05$ to NAFL. Data are mean \pm SEM.

We next examined if changes in IHL content in the complete group were associated with changes in other outcome parameters measured. We interestingly observed that a decrease in IHL content with exercise training correlated positively with changes in fat mass $(r=0.482, p=0.003)$ and negatively with an increase in $\mathrm{VO}_{2 \max }$ $(r=-0.347, p=0.041$ ) (figure 2).

Hepatic energy status defined as ATP/Total Phosphorus ratio did not show a significant time $(p=0.890)$, group $(p=0.357)$ or time*group $(p=0.193)$ effect upon exercise training. Pre-training, ATP/Total Phosphorus was $15.5 \pm 0.4,15.8 \pm 0.6$ and $15.8 \pm 0.4 \%$ in CTRL, NAFL and T2DM, respectively. Post-training, ATP/Total Phosphorus values were $14.8 \pm 0.7,16.5 \pm 0.6$ and $15.7 \pm 0.4 \%$ in CTRL, NAFL and T2DM, respectively. 


\section{INSULIN SENSITIVITY}

Data on insulin sensitivity is displayed in table 2 . There was a significant group $(p<0.001)$, but no time $(p=0.131)$ or time*group $(p=0.176)$ effect in basal EGP, with basal EGP being significantly higher in T2DM patients compared to CTRL $(p<0.001)$ and NAFL subjects $(p<0.001$ ). Also relative suppression of EGP (delta EGP \%) during low-dose insulin infusion showed a significant group $(p<0.001)$, but no time $(p=0.337)$ or time*group $(p=0.584)$ effect upon exercise training $(-70.1 \pm 3.5$ to $76.5 \pm 6.2,-41.0 \pm 6.0$ to $-40.7 \pm 6.3$ and $-42.1 \pm 1.9$ to $-43.7 \pm 2.4 \%$ for CTRL, NAFL and T2DM, respectively). Delta EGP \% during low-dose insulin infusion was significantly more pronounced in CTRL subjects in comparison to NAFL $(p<0.001)$ and T2DM $(p<0.001)$, with the latter two being comparable $(p=0.976)$. EGP with high-dose insulin infusion was almost completely suppressed in all groups, both pre- and post-exercise training (table 2).
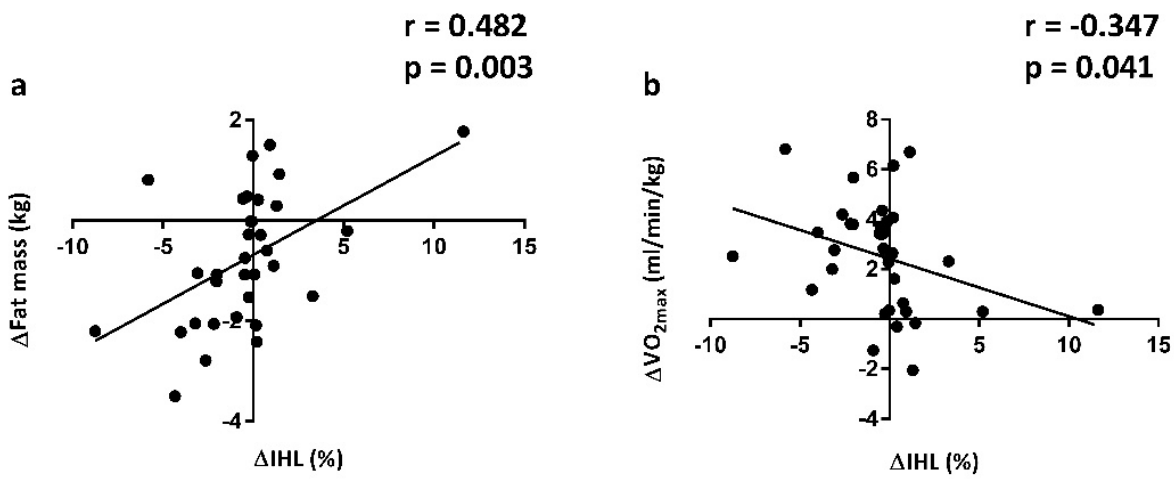

Figure 2. Comparison between the change in intrahepatic lipid content $(\Delta \mathrm{HL})$ and changes in (a) fat mass ( $\Delta$ fat mass, $n=35)$ and (b) maximum aerobic capacity $\left(\Delta \mathrm{VO}_{2 \max }, \mathrm{n}=35\right)$ upon exercise training. $\mathrm{IHL}$ is expressed as the relative intensity of the $\mathrm{CH}_{2}$ peak, compared to the unsuppressed water resonance.

Skeletal muscle insulin sensitivity was examined by measuring insulin-stimulated glucose disposal (delta $R_{d}$ ). Upon low-dose insulin infusion, no effect of exercise training on delta $R_{d}$ was observed, although there was a significant group effect $(p<0.001)$, with groups responding differently on low-dose insulin infusion (table 2 ). 


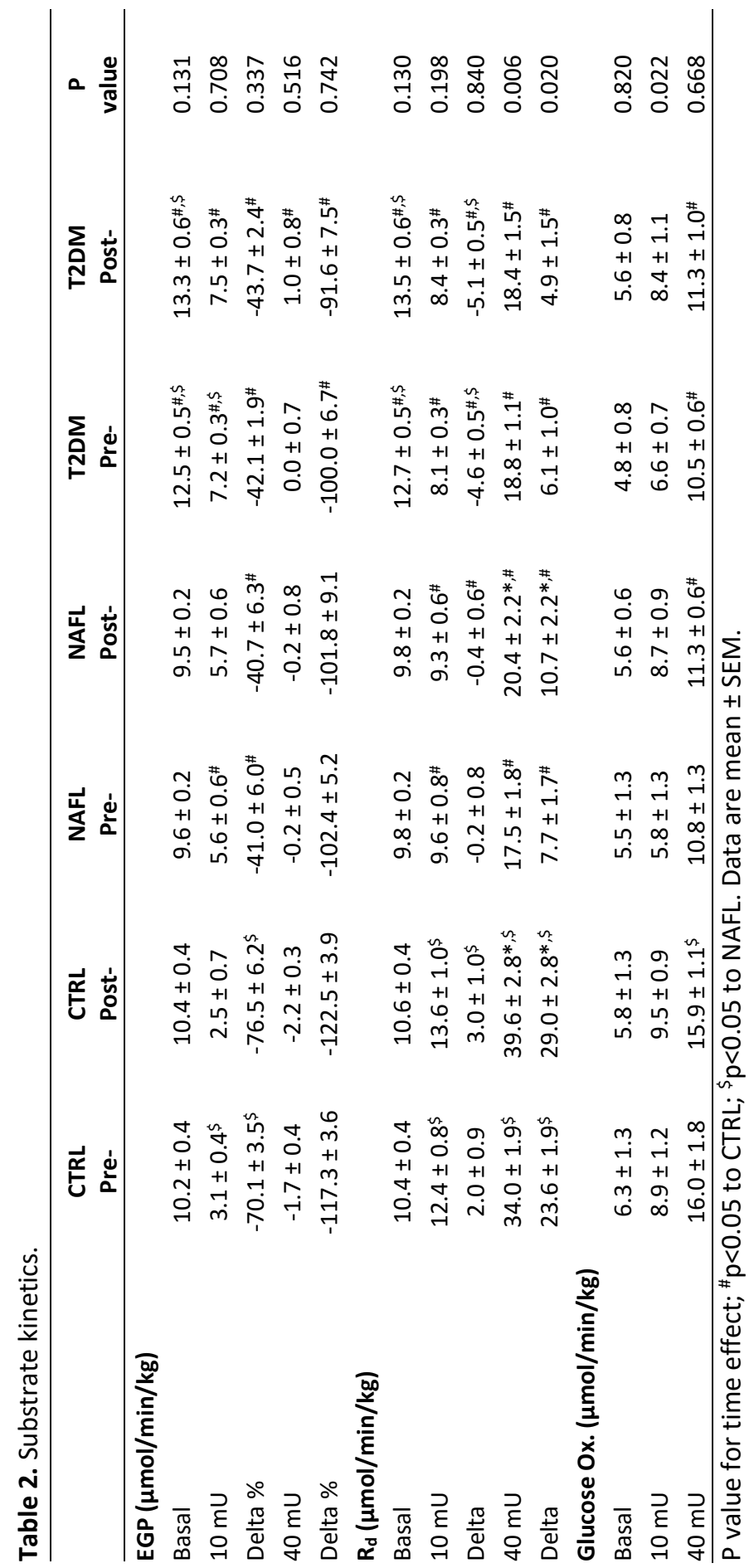




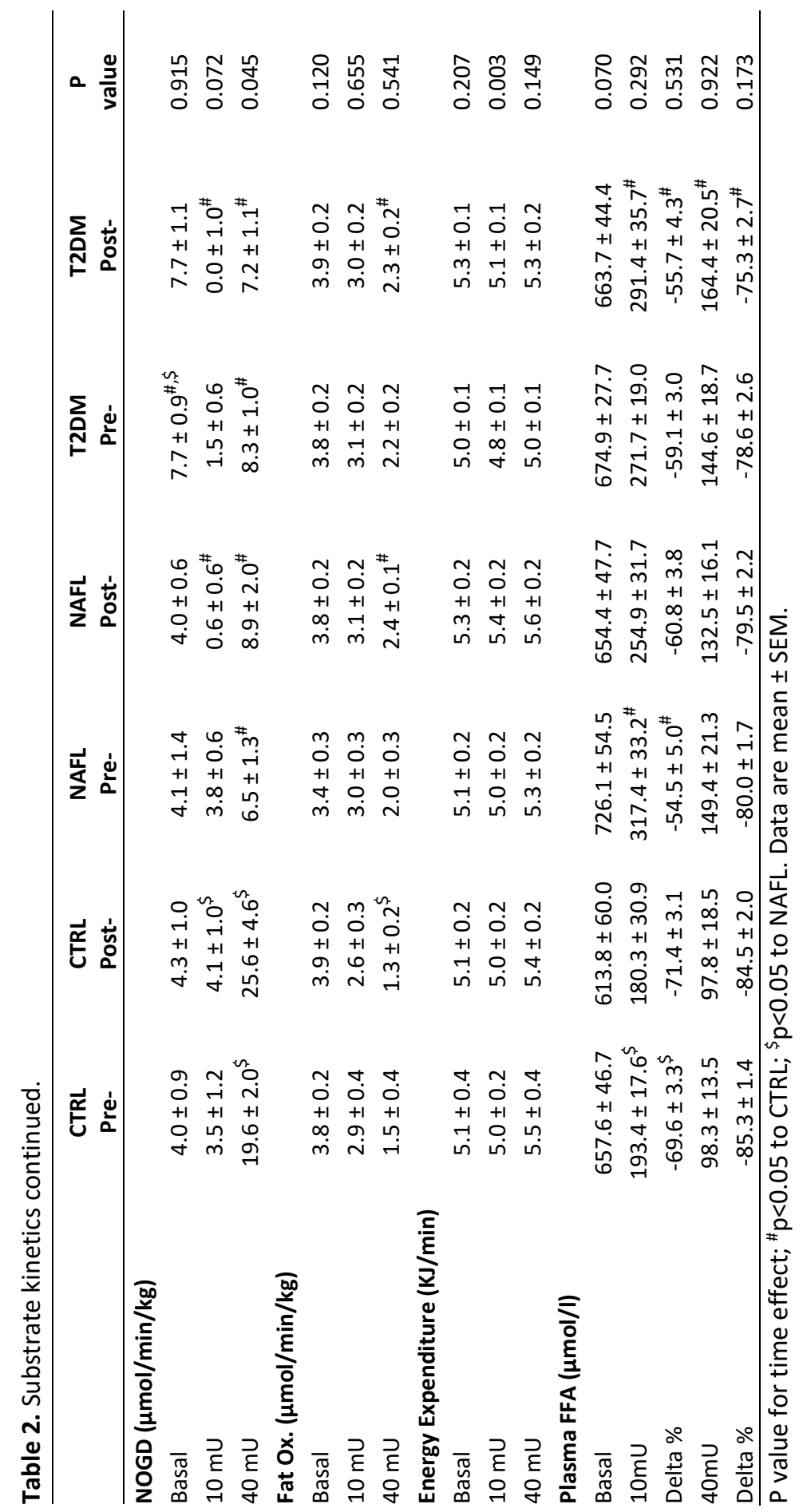


Interestingly, whereas in CTRL the low-dose of insulin already increased $R_{d}$, glucose disposal was not increased in T2DM and NAFL (table 2). In T2DM patients, this lack of effect of a low-dose insulin infusion on $R_{d}$ is generally explained by a glucose mass effect, as basal $R_{d}$ is measured under hyperglycemic conditions. However, the lack of effect of low-dose insulin infusion on $R_{d}$ in the normoglycemic NAFL subjects indicates severe skeletal muscle insulin resistance in these subjects. Indeed, when using a high-dose insulin infusion, insulin-stimulated $R_{d}$ was significantly higher in CTRL subjects compared to NAFL $(p<0.001)$ and T2DM $(p<0.001)$ patients, with no significant differences between the latter two groups (table 2 ). In contrary to the low-dose insulin infusion, there was a significant time $(p=0.020)$, group $(p<0.001)$ and time*group ( $p=0.022$ ) effect in delta $R_{d}$ upon $40 \mathrm{mU} / \mathrm{m}^{2} / \mathrm{min}$ insulin infusion after exercise training (table 2). Exercise training significantly increased delta $R_{d}$ in CTRL (from $23.6 \pm 1.9$ to $29.0 \pm 2.8 \mu \mathrm{mol} / \mathrm{min} / \mathrm{kg}, \mathrm{p}=0.031$ ) and NAFL (from $7.7 \pm 1.7$ to $10.7 \pm 2.2 \mu \mathrm{mol} / \mathrm{min} / \mathrm{kg}, \mathrm{p}=0.012$ ) subjects, but peripheral insulin sensitivity was not significantly changed in T2DM patients (from $6.1 \pm 1.0$ to $4.9 \pm 1.5 \mu \mathrm{mol} / \mathrm{min} / \mathrm{kg}$, $p=0.475$ ) (figure 3 ). The time effect in delta $R_{d}$ during high-dose insulin infusion was mainly caused by a significant increase in NOGD during high-dose insulin infusion over time (table $2, p=0.045)$, with a close to significant time* group effect ( $p=0.059)$, whereas glucose oxidation during high-dose insulin infusion was not significantly changed over time (table 2, $\mathrm{p}=0.668$ ).

\section{SUBSTRATE OXIDATION AND ENERGY EXPENDITURE}

There was no significant time $(p=0.820)$, group $(p=0.769)$ or time*group $(p=0.698)$ effect for basal glucose oxidation (table 2). Glucose oxidation during low-dose insulin infusion increased significantly over time $(p=0.022)$, with an average increase over time of $21.1 \%$ (from $7.1 \pm 0.5$ to $8.6 \pm 0.5 \mu \mathrm{mol} / \mathrm{min} / \mathrm{kg}$ ). Glucose oxidation upon high-dose insulin stimulation showed a significant group $(p<0.001)$, but no time or time*group effect, with glucose oxidation being significantly higher in the CTRL subjects compared to NAFL $(p=0.002)$ and T2DM $(p=0.001)$ patients (table 2). Basal fat oxidation and fat oxidation during low-dose insulin infusion revealed no significant time, group or time*group effects (table 2). Fat oxidation upon high-dose insulin stimulation showed a significant group $(p=0.019)$, but no 
time or time*group effect, with fat oxidation being significantly lower in the CTRL subjects compared to NAFL $(p=0.050)$ and T2DM $(p=0.018)$ patients (table 2$)$.

There was no time $(p=0.207)$, group $(p=0.965)$ or time*group $(p=0.622)$ effect in basal energy expenditure. Energy expenditure upon low-dose insulin infusion showed a significant time effect $(p=0.003)$, with an average increase over time of $0.3 \mathrm{KJ} / \mathrm{min}$ (from $4.9 \pm 0.1$ to $5.2 \pm 0.1 \mathrm{KJ} / \mathrm{min}$ ). Energy expenditure with high-dose insulin infusion did not show any significant effect. The increase in energy expenditure during low-dose insulin infusion correlated positively with changes in fat free mass upon exercise training $(r=0.461, p=0.008)$.

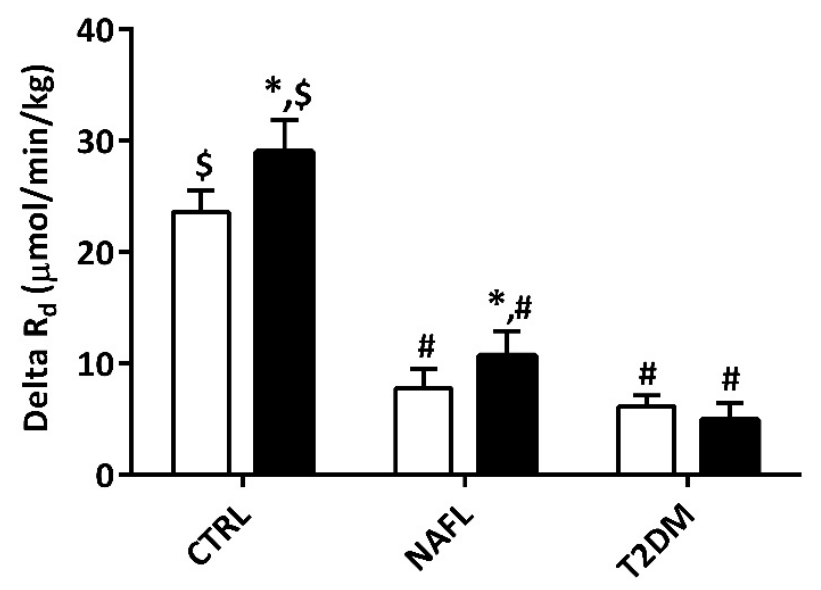

Figure 3. Insulin- stimulated glucose disposal (delta $\mathrm{Rd}_{\mathrm{d}}$ ) with $40 \mathrm{mU} / \mathrm{m}^{2} / \mathrm{min}$ insulin infusion pre- (white columns) and post- (black columns) exercise training. $\mathrm{n}=9$ for $\mathrm{CTRL}, \mathrm{n}=8$ for NAFL, $\mathrm{n}=11$ for T2DM. $* \mathrm{p}<0.05$ from baseline; ${ }^{\#} \mathrm{p}<0.05$ to CTRL; $\$ p<0.05$ to NAFL. Data are mean \pm SEM.

\section{BLOOD SAMPLE ANALYSIS}

By definition, there was a significant group effect $(p<0.001)$, but no time $(p=0.887)$ or time*group ( $p=0.576$ ) effect for FPG, which was significantly higher in the T2DM patients than in CTRL $(p<0.001)$ and NAFL $(p<0.001)$ subjects (table 1$)$. There was no time $(p=0.240)$, group $(p=0.145)$ or time*group $(p=0.537)$ effect for plasma TG. Free glycerol levels showed a significant time $(p=0.037)$, but no group or time*group effect with exercise training, and decreased with $13.2 \%$ on average over time (from $0.12 \pm 0.07$ to $0.11 \pm 0.07 \mathrm{mmol} / \mathrm{l}$ ). Fasting plasma FFA levels were similar across groups and were not significantly changed upon exercise training 
(from $657.6 \pm 46.7$ to $613.8 \pm 60.0,726.1 \pm 54.5$ to $654.4 \pm 47.7$ and $674.9 \pm 27.7$ to $663.7 \pm 44.4 \mu \mathrm{mol} / \mathrm{I}$ for CTRL, NAFL and T2DM, respectively, table 2).

To evaluate the insulin sensitivity of adipose tissue, the relative suppression of plasma FFA upon insulin infusion was determined. We found that with low-dose insulin infusion a significant group $(p=0.009)$, but no time $(p=0.531)$ or time*group $(p=0.291)$ effect was found. Thus, CTRL subjects were significantly better in suppressing plasma FFA levels than NAFL $(p=0.020)$ and T2DM $(p=0.018)$ patients, suggesting differences in adipose tissue insulin sensitivity (table 2 ). A significant group $(p=0.020)$, but no time $(p=0.173)$ or time* $\operatorname{group}(p=0.526)$ effect in relative suppression of plasma FFA was also visible with high-dose insulin infusion, with CTRL subjects having a significantly more profound suppression than T2DM patients $(p=0.017$, table 2$)$.

Table 3. Mitochondrial density protein content (AU).

\begin{tabular}{llllllll}
\hline & $\begin{array}{l}\text { CTRL } \\
\text { Pre- }\end{array}$ & $\begin{array}{l}\text { CTRL } \\
\text { Post- }\end{array}$ & $\begin{array}{l}\text { NAFL } \\
\text { Pre- }\end{array}$ & $\begin{array}{l}\text { NAFL } \\
\text { Post- }\end{array}$ & $\begin{array}{l}\text { T2DM } \\
\text { Pre- }\end{array}$ & $\begin{array}{l}\text { T2DM } \\
\text { Post- }\end{array}$ & $\begin{array}{l}\text { P } \\
\text { value }\end{array}$ \\
\hline Complex I & $0.96 \pm$ & $1.13 \pm$ & $1.00 \pm$ & $1.17 \pm$ & $0.87 \pm$ & $0.99 \pm$ & 0.118 \\
& 0.13 & 0.16 & 0.13 & 0.23 & 0.10 & 0.12 & \\
Complex II & $0.96 \pm$ & $1.07 \pm$ & $0.98 \pm$ & $1.06 \pm$ & $0.96 \pm$ & $1.01 \pm$ & 0.200 \\
& 0.10 & 0.12 & 0.11 & 0.15 & 0.10 & 0.10 & \\
Complex III & $1.03 \pm$ & $1.09 \pm$ & $1.04 \pm$ & $0.99 \pm$ & $0.90 \pm$ & $1.01 \pm$ & 0.368 \\
& 0.08 & 0.06 & 0.13 & 0.13 & 0.06 & 0.05 & \\
Complex IV & $1.01 \pm$ & $1.07 \pm$ & $1.02 \pm$ & $1.01 \pm$ & $0.91 \pm$ & $1.04 \pm$ & 0.303 \\
& 0.08 & 0.07 & 0.10 & 0.15 & 0.06 & 0.09 & \\
Complex V & $1.06 \pm$ & $1.14 \pm$ & $0.96 . \pm$ & $1.06 \pm$ & $0.89 \pm$ & $0.97 \pm$ & 0.040 \\
& 0.06 & 0.05 & 0.10 & 0.17 & 0.05 & 0.05 & \\
Average of the & $1.02 \pm$ & $1.10 \pm$ & $1.04 \pm$ & $1.13 \pm$ & $0.90 \pm$ & $1.01 \pm$ & 0.016 \\
complexes & 0.07 & 0.05 & 0.11 & 0.12 & 0.05 & 0.06 & \\
\hline P value for tim & & & &
\end{tabular}

$\mathrm{P}$ value for time effect; ${ }^{\#} \mathrm{p}<0.05$ to $\mathrm{CTRL} ;{ }^{\zeta} \mathrm{p}<0.05$ to NAFL. Data are mean \pm SEM.

\section{MITOCHONDRIAL DENSITY}

Mitochondrial density was evaluated by measuring protein content of 5 structural subunits of the 5 distinct complexes of the electron transport chain. There was no time, group or time*group effect for complex I, II, III and IV (table 3). A significant time $(p=0.040)$, but no group $(p=0.274)$ or time*group $(p=0.994)$ effect for complex 
$\mathrm{V}$ was visible after exercise training. Thus, average complex $\mathrm{V}$ protein content significantly increased over time from $0.96 \pm 0.04$ to $1.05 \pm 0.06 \mathrm{AU}$. Interestingly, also the average protein content of these complexes revealed a significant time $(p=0.016)$, but no group $(p=0.365)$ or time*group $(p=0.927)$ effect upon exercise training. Average OXPHOS protein content significantly increased over time from $0.98 \pm 0.04$ to $1.07 \pm 0.04 \mathrm{AU}$.

\section{DISCUSSION}

Lifestyle intervention is considered the standard treatment for NAFL, and diet intervention studies have been effective in lowering $\mathrm{IHL}$ content ${ }^{9-12}$. Adding exercise training to a dietary restriction can have an additive effect in reducing hepatic fat content, and therefore exercise might be an effective way to decrease $\mathrm{IHL}$ content ${ }^{12}$. Until now, studies that investigated the effect of exercise training on $\mathrm{IHL}$ used quite a range of training paradigms and focused either on only one of the following (sub)populations; overweight/obese healthy subjects, subjects with NAFL or T2DM patients with NAFL. This most likely explains lack of consensus in the field on the exact effects of exercise training on IHL content in these groups. Hence we here measured the effects of a well-controlled exercise training intervention on $\mathrm{IHL}$ content and insulin sensitivity, without dietary restrictions and without targeted weight loss, in healthy overweight/obese CTRL subjects, NAFL subjects and T2DM patients all undergoing the same individualized training program. We observed that 12 weeks of supervised exercise training without targeted weight loss significantly decreased IHL content in NAFL subjects, but intriguingly not in CTRL subjects and T2DM patients. Interestingly, hepatic insulin sensitivity was not improved in any of the groups, whereas in CTRL and NAFL subjects peripheral insulin sensitivity significantly improved after exercise training.

The approximately $23 \%$ decrease in IHL content in NAFL subjects is in line with previous results reported upon aerobic and resistance exercise training programs ${ }^{19}$ 22. Van der Heijden et al. ${ }^{19}$ reported a $37 \%$ reduction in IHL after 12 weeks of aerobic exercise training in obese, Hispanic adolescents with elevated liver fat concentrations. Eight weeks of resistance exercise reduced IHL content with $13 \%$ in obese, middle-aged subjects with $\mathrm{NAFL}^{22}$. Our finding that $\mathrm{IHL}$ did not 
significantly decrease in CTRL subjects may be due to the lower IHL content at the onset of the study, prior to the training program, and therefore absolute changes in IHL might be smaller than in people with NAFL. Thus, we observed that IHL content decreased by $21 \%$ in CTRL (from $1.9 \pm 0.4$ to $1.5 \pm 0.3 \%$ ), but this effect did not reach statistical significance. Previously, one study reported beneficial effects of exercise training in 50 obese people without $\mathrm{NAFL}^{23}$, while two other intervention studies did not find an exercise lowering effect on IHL in 10 and 20 obese subjects ${ }^{24,25}$. Nevertheless, given the average decrease of $21 \%$ observed in our study, exercise training might be effective to prevent a potential increase in liver fat content in overweight/obese people over time. In contrast, however, in T2DM patients IHL content was completely unaffected by exercise training (from $8.9 \pm 3.3$ to $9.9 \pm 4.1 \%$ ). Bacchi et al. ${ }^{8}$ reported decreased $\mathrm{IHL}$ content after 4 months of aerobic and resistance exercise training in T2DM patients with NAFL. However, here we included people with T2DM without selecting for IHL content. Interestingly, the lack of effect of exercise training on IHL content in T2DM patients coincides with a lack of effect on insulin sensitivity in this group, suggesting that an effect on insulin sensitivity may be required to affect IHL content.

Higher levels of physical activity have been associated with a lower IHL content ${ }^{18,34}$. Kantartzis et al. ${ }^{17}$ reported that cardiorespiratory fitness at baseline, independently of total adiposity, body fat distribution and exercise intensity, determines the change in liver fat content after a lifestyle intervention, suggesting that fitness and liver fat are causally related to each other. Here we found that changes in $\mathrm{IHL}$ content over time correlated negatively with changes in $\mathrm{VO}_{2 \max }$ (i.e. the more $\mathrm{VO}_{2 \max }$ increased upon exercise training, the larger the drop in $\mathrm{IHL}$ ) and positively with changes in fat mass (a larger decrease in fat mass correlates with a larger decrease in $\mathrm{IHL}$ ), whereas changes in $\mathrm{VO}_{2 \max }$ did not correlate with changes in fat mass. These data suggest that both $\mathrm{VO}_{2 \max }$ and fat mass are important determinants of exercise training mediated changes in $\mathrm{IHL}$ content.

Next to IHL, we measured the effect of exercise training on insulin sensitivity. It has been previously reported that NAFL subjects suffer from impaired hepatic, adipose tissue and skeletal muscle insulin sensitivity when compared to obese subjects without NAFL ${ }^{4-6}$. A significant improvement in whole body insulin sensitivity using HOMA-IR after 8 weeks of resistance exercise has been reported by Hallsworth et 
al. ${ }^{22}$, whereas Johnson et al. ${ }^{20}$ did not observe an improvement in HOMA-IR after 4 weeks of aerobic exercise training. To the best of our knowledge, this is the first study to investigate tissue specific effects of exercise training on insulin sensitivity in people with elevated IHL content. We found that in CTRL and NAFL subjects, exercise training positively affected skeletal muscle insulin sensitivity, but not hepatic or adipose tissue insulin sensitivity. The lack of effect of exercise training on hepatic insulin sensitivity in NAFL and CTRL subjects is interesting and suggests that, in contrast to what is commonly believed, a reduction in $\mathrm{IHL}$ content does not necessarily results in improved hepatic insulin sensitivity.

High fasting and postprandial FFA levels are believed to be a main contributor of elevated IHL content ${ }^{35,36}$. However, it is not clear whether exercise training influences adipose tissue insulin sensitivity and hence adipose tissue lipolysis directly ${ }^{24,37}$, and if this could contribute to the exercise training lowering effect on IHL content. In that respect, the lack of effect of exercise training on fasting FFA levels and insulin-stimulated suppression of plasma FFA in this study suggests that an improvement in adipose tissue insulin sensitivity is not necessary to reduce IHL content. Therefore, our study results may imply that the reduction of IHL upon exercise training is not due to alterations in adipose tissue fatty acid metabolism.

Surprisingly, we did not observe any significant improvement in hepatic, adipose tissue or peripheral insulin sensitivity in the T2DM patients. Previously, we showed that 12 weeks of combined exercise training improved insulin-stimulated glucose disposal by $63 \%$ in a group of T2DM patients with comparable characteristics ${ }^{14}$. It should be noted, though, that in the study of Meex et al. (for reasons unexplained) the increase in $\mathrm{VO}_{2 \max }$ in the T2DM patients was more pronounced than the increase we observed in our T2DM patients (13\% increase in the study of Meex et al., $5 \%$ in this study). One of the putative cellular mechanisms contributing to attenuated peripheral insulin sensitivity might be impaired mitochondrial oxidative capacity ${ }^{38}$. Interestingly, in the study of Meex et al. $^{14}$, the increase in insulinstimulated glucose uptake occurred in parallel with improvements in $\mathrm{VO}_{2 \max }$, mitochondrial content and mitochondrial function. In the study of Meex et al., these adaptive responses translated in an exercise training mediated restoration of oxidative glucose uptake post-exercise training, compared to obese normoglycemic controls. Although we did not measure skeletal muscle mitochondrial function in 
the present study, the mild increase in $\mathrm{VO}_{2 \max }$ in our T2DM patients may reflect a more modest mitochondrial response in the present study compared to the study by Meex et al.. Therefore we did analyze mitochondrial content pre- and postexercise training by measuring the protein content of 5 distinct structural proteins in the electron transport chain. We found that exercise training increased protein content of complex $\mathrm{V}$ and average OXPHOS protein content over time, indicating that exercise training did increase mitochondrial content in our subjects.

So, the reason for this lack of adaptive response on insulin sensitivity is currently unknown, but does not involve compliance (96, 95 and $97 \%$ for CTRL, NAFL and T2DM, respectively) or subject characteristics (as those were similar to the study of Meex et al. $\left.^{14}\right)$. We cannot rule out that intrinsic defects of the mitochondria compromised the mitochondrial function in our T2DM patients ${ }^{39,40}$. Furthermore, since $\mathrm{VO}_{2 \max }$ only mildly improved, other factors like (a lack of exercise training effect on) cardiac output and/or capillary density, which were not measured in this study, might underlie the lack of adaptive response on insulin sensitivity in our study. On the other hand, it is known that $15-20 \%$ of individuals fail to improve their glucose homeostasis, insulin sensitivity and/or skeletal muscle mitochondrial function after supervised exercise training interventions ${ }^{41,42}$, and Stephens and Sparks recently reviewed that epigenetic modifications may dictate the exercise training response ${ }^{43}$. Therefore more in depth analysis, including genotyping for genes that may affect exercise training responsiveness on insulin sensitivity, might be needed to unravel this lack of exercise training response.

In conclusion, exercise training significantly decreased intrahepatic lipid content in NAFL subjects. Remarkably, however, exercise training induced changes in IHL content in NAFL subjects did not result in improved hepatic insulin sensitivity, while skeletal muscle insulin sensitivity was improved. Our data suggest that exercise training may specifically be of interest to reduce IHL in NAFL subjects, especially in whom exercise training indeed improves $\mathrm{VO}_{2 \max }$ and/or reduces fat mass. 


\section{REFERENCES}

1. Ng, M., et al. Global, regional, and national prevalence of overweight and obesity in children and adults during 1980-2013: a systematic analysis for the Global Burden of Disease Study 2013. Lancet 384, 766-781 (2014).

2. Armstrong, M.J., et al. Abdominal subcutaneous adipose tissue insulin resistance and lipolysis in patients with non-alcoholic steatohepatitis. Diabetes Obes Metab 16, 651-660 (2014).

3. Utzschneider, K.M. \& Kahn, S.E. Review: The role of insulin resistance in nonalcoholic fatty liver disease. J Clin Endocrinol Metab 91, 4753-4761 (2006).

4. Fabbrini, E., et al. Intrahepatic fat, not visceral fat, is linked with metabolic complications of obesity. Proc Natl Acad Sci U S A 106, 15430-15435 (2009).

5. Korenblat, K.M., Fabbrini, E., Mohammed, B.S. \& Klein, S. Liver, muscle, and adipose tissue insulin action is directly related to intrahepatic triglyceride content in obese subjects. Gastroenterology 134, 1369-1375 (2008).

6. Hwang, J.H., et al. Increased intrahepatic triglyceride is associated with peripheral insulin resistance: in vivo MR imaging and spectroscopy studies. Am J Physiol Endocrinol Metab 293, E1663-1669 (2007).

7. Gaggini, M., et al. Non-alcoholic fatty liver disease (NAFLD) and its connection with insulin resistance, dyslipidemia, atherosclerosis and coronary heart disease. Nutrients 5, 1544-1560 (2013).

8. Bacchi, E., et al. Both resistance training and aerobic training reduce hepatic fat content in type 2 diabetic subjects with NAFLD (The RAED2 randomized trial). Hepatology (2013).

9. Shah, K., et al. Diet and exercise interventions reduce intrahepatic fat content and improve insulin sensitivity in obese older adults. Obesity (Silver Spring) 17, 2162-2168 (2009).

10. Larson-Meyer, D.E., et al. Effect of calorie restriction with or without exercise on insulin sensitivity, beta-cell function, fat cell size, and ectopic lipid in overweight subjects. Diabetes Care 29, 1337-1344 (2006).

11. Lazo, M., et al. Effect of a 12-month intensive lifestyle intervention on hepatic steatosis in adults with type 2 diabetes. Diabetes Care 33, 21562163 (2010). 
12. Goodpaster, B.H., et al. Effects of diet and physical activity interventions on weight loss and cardiometabolic risk factors in severely obese adults: a randomized trial. Jama 304, 1795-1802 (2010).

13. Colberg, S.R., et al. Exercise and type 2 diabetes: the American College of Sports Medicine and the American Diabetes Association: joint position statement. Diabetes Care 33, e147-167 (2010).

14. Meex, R.C., et al. Restoration of muscle mitochondrial function and metabolic flexibility in type 2 diabetes by exercise training is paralleled by increased myocellular fat storage and improved insulin sensitivity. Diabetes 59, 572-579 (2010).

15. Bruce, C.R., Kriketos, A.D., Cooney, G.J. \& Hawley, J.A. Disassociation of muscle triglyceride content and insulin sensitivity after exercise training in patients with Type 2 diabetes. Diabetologia 47, 23-30 (2004).

16. Misra, A., et al. Effect of supervised progressive resistance-exercise training protocol on insulin sensitivity, glycemia, lipids, and body composition in Asian Indians with type 2 diabetes. Diabetes Care 31, 1282-1287 (2008).

17. Kantartzis, K., et al. High cardiorespiratory fitness is an independent predictor of the reduction in liver fat during a lifestyle intervention in nonalcoholic fatty liver disease. Gut 58, 1281-1288 (2009).

18. Perseghin, G., et al. Habitual physical activity is associated with intrahepatic fat content in humans. Diabetes Care 30, 683-688 (2007).

19. van der Heijden, G.J., et al. A 12-week aerobic exercise program reduces hepatic fat accumulation and insulin resistance in obese, Hispanic adolescents. Obesity (Silver Spring) 18, 384-390 (2010).

20. Johnson, N.A., et al. Aerobic exercise training reduces hepatic and visceral lipids in obese individuals without weight loss. Hepatology 50, 1105-1112 (2009).

21. Sullivan, S., Kirk, E.P., Mittendorfer, B., Patterson, B.W. \& Klein, S. Randomized trial of exercise effect on intrahepatic triglyceride content and lipid kinetics in nonalcoholic fatty liver disease. Hepatology 55, 1738-1745 (2012).

22. Hallsworth, K., et al. Resistance exercise reduces liver fat and its mediators in non-alcoholic fatty liver disease independent of weight loss. Gut 60, 1278-1283 (2011). 
23. Finucane, F.M., et al. The effects of aerobic exercise on metabolic risk, insulin sensitivity and intrahepatic lipid in healthy older people from the Hertfordshire Cohort Study: a randomised controlled trial. Diabetologia 53, 624-631 (2010).

24. Shojaee-Moradie, F., et al. Exercise training reduces fatty acid availability and improves the insulin sensitivity of glucose metabolism. Diabetologia 50, 404-413 (2007).

25. Devries, M.C., Samjoo, I.A., Hamadeh, M.J. \& Tarnopolsky, M.A. Effect of endurance exercise on hepatic lipid content, enzymes, and adiposity in men and women. Obesity (Silver Spring) 16, 2281-2288 (2008).

26. Kuipers, H., Verstappen, F.T., Keizer, H.A., Geurten, P. \& van Kranenburg, G. Variability of aerobic performance in the laboratory and its physiologic correlates. Int J Sports Med 6, 197-201 (1985).

27. Bilet, L., et al. Acute exercise does not decrease liver fat in men with overweight or NAFLD. Sci Rep 5, 9709 (2015).

28. DeFronzo, R.A., Tobin, J.D. \& Andres, R. Glucose clamp technique: a method for quantifying insulin secretion and resistance. Am J Physio/ 237, E214-223 (1979).

29. Mensink, M., Blaak, E.E., van Baak, M.A., Wagenmakers, A.J. \& Saris, W.H. Plasma free Fatty Acid uptake and oxidation are already diminished in subjects at high risk for developing type 2 diabetes. Diabetes 50, 2548-2554 (2001).

30. Peronnet, F. \& Massicotte, D. Table of nonprotein respiratory quotient: an update. Can J Sport Sci 16, 23-29 (1991).

31. Ackermans, M.T., et al. The quantification of gluconeogenesis in healthy men by (2) $\mathrm{H} 2 \mathrm{O}$ and [2-(13)C]glycerol yields different results: rates of gluconeogenesis in healthy men measured with (2) $\mathrm{H} 2 \mathrm{O}$ are higher than those measured with [2-(13)C]glycerol. The Journal of clinical endocrinology and metabolism 86, 2220-2226 (2001).

32. Steele, R. Influences of glucose loading and of injected insulin on hepatic glucose output. Ann N Y Acad Sci 82, 420-430 (1959).

33. Bergstrom, J., Hermansen, L., Hultman, E. \& Saltin, B. Diet, muscle glycogen and physical performance. Acta Physiol Scand 71, 140-150 (1967). 
34. Church, T.S., et al. Association of cardiorespiratory fitness, body mass index, and waist circumference to nonalcoholic fatty liver disease. Gastroenterology 130, 2023-2030 (2006).

35. Sanyal, A.J., et al. Nonalcoholic steatohepatitis: association of insulin resistance and mitochondrial abnormalities. Gastroenterology 120, 11831192 (2001).

36. Donnelly, K.L., et al. Sources of fatty acids stored in liver and secreted via lipoproteins in patients with nonalcoholic fatty liver disease. J Clin Invest 115, 1343-1351 (2005).

37. Lange, K.H., et al. Endurance training and GH administration in elderly women: effects on abdominal adipose tissue lipolysis. American journal of physiology. Endocrinology and metabolism 280, E886-897 (2001).

38. Malin, S.K., et al. Insulin sensitivity and metabolic flexibility following exercise training among different obese insulin-resistant phenotypes. American journal of physiology. Endocrinology and metabolism 305, E12921298 (2013).

39. Mogensen, M., et al. Mitochondrial respiration is decreased in skeletal muscle of patients with type 2 diabetes. Diabetes 56, 1592-1599 (2007).

40. Phielix, E., et al. Lower intrinsic ADP-stimulated mitochondrial respiration underlies in vivo mitochondrial dysfunction in muscle of male type 2 diabetic patients. Diabetes 57, 2943-2949 (2008).

41. Bajpeyi, S., et al. Effect of exercise intensity and volume on persistence of insulin sensitivity during training cessation. J Appl Physiol (1985) 106, 10791085 (2009).

42. Sparks, L.M., et al. Nine months of combined training improves ex vivo skeletal muscle metabolism in individuals with type 2 diabetes. The Journal of clinical endocrinology and metabolism 98, 1694-1702 (2013).

43. Stephens, N.A. \& Sparks, L.M. Resistance to the beneficial effects of exercise in type 2 diabetes: are some individuals programmed to fail? The Journal of clinical endocrinology and metabolism 100, 43-52 (2015). 




\section{CHAPTER 6}

\section{EXERCISE TRAINING-INDUCED EFFECTS ON ABDOMINAL SUBCUTANEOUS ADIPOSE TISSUE GENE EXPRESSION IN OBESE HUMANS}

Bram Brouwers*, Rudi Stinkens*, Johan W.E. Jocken, Ellen E. Blaak, Matthijs K.C. Hesselink, Patrick Schrauwen, Gijs Goossens

In preparation for submission

* These authors contributed equally to this work 


\section{ABSTRACT}

Rodent studies have indicated that physical exercise may improve adipose tissue function. Here, we aimed to determine the effects of a 12 week exercise training program on abdominal subcutaneous adipose tissue gene expression of markers related to adipose tissue function in well-phenotyped, metabolically healthy and metabolically compromised obese subjects.

Twenty-one obese men (14 metabolically compromised (non-alcoholic fatty liver and/or type 2 diabetes) and 7 metabolically healthy) participated in a 12 week supervised, progressive, combined exercise training program. At baseline and after intervention, abdominal subcutaneous adipose tissue biopsies were collected to determine adipokine expression and gene expression of markers for lipolysis, inflammation, browning and mitochondrial biogenesis/function.

At baseline, adipose tissue gene expression of HSL $(P=0.005), C G I-58(P<0.001)$ and PGC-1 $\alpha(P=0.037)$ as well as peripheral $(P<0.001)$, hepatic $(P<0.001)$ and adipose tissue insulin sensitivity $(P=0.048)$ were significantly lower, while fasting plasma glucose $(P=0.009)$ and HOMA-IR $(P<0.002)$ were significantly higher in the metabolically compromised as compared to metabolically healthy subjects. The exercise training program, which increased maximal aerobic capacity $(P<0.001)$ and muscle strength $(P<0.001)$ and slightly reduced body fat mass $(P=0.021)$, did not affect abdominal subcutaneous adipose tissue gene expression of markers for mitochondrial biogenesis and function, browning, lipolysis, inflammation and adipokines, irrespective of baseline metabolic status.

In conclusion, a 12 week supervised, progressive exercise training program did not affect abdominal subcutaneous adipose tissue gene expression of markers related to adipose tissue function in obese subjects, irrespective of the metabolic status. 


\section{INTRODUCTION}

The obesity epidemic is paralleled by a profound increase in the prevalence of obesity-related diseases, including type 2 diabetes mellitus (T2DM), non-alcoholic fatty liver disease (NAFLD), cardiovascular disease and certain types of cancer ${ }^{1}$. A sedentary lifestyle is a major contributor to obesity-related complications. In line, increased habitual physical activity and exercise training may have beneficial effects on insulin sensitivity and glucose homeostasis in obese, insulin resistant and T2DM patients ${ }^{2-4}$. Therefore, increasing physical activity is a recommended lifestyle modification in the prevention and treatment of obesity-related disorders, including $\mathrm{T}^{2} \mathrm{DM}^{5}$.

Since skeletal muscle is responsible for the majority of glucose disposal, adaptations in skeletal muscle metabolism are thought to play a central role in the exercise training induced improvement of insulin sensitivity. Adipose tissue dysfunction in obesity, however, represents one of the initial steps in the development of insulin resistance and obesity-related diseases ${ }^{6,7}$. The reason for this is that enlargement of fat cells (adipocyte hypertrophy), due to excessive energy storage, promotes low-grade inflammation and decreases the capacity of adipose tissue to buffer the daily lipid fluxes. This leads to lipid accumulation in nonadipose tissues, such as skeletal muscle and the liver, thereby accelerating the development and progression of insulin resistance and chronic metabolic diseases ${ }^{6-}$ 8 .

Interestingly, there is evidence that exercise training may improve adipose tissue function. In fact, several rodent studies have demonstrated that exercise training increased mitochondrial biogenesis ${ }^{9,10}$ and function ${ }^{11-13}$, induced browning of white adipose tissue $\mathrm{e}^{9,10,12,14,15}$ and altered adipokine expression ${ }^{16,17}$, as reviewed ${ }^{18}$. However, human studies that have investigated the effects of exercise training on the adipose tissue phenotype are scarce. Nevertheless, exercise training may increase gene expression of peroxisome proliferator-activated receptor-gamma coactivator-1 alpha (PGC- $1 \alpha)^{19}$ and markers of oxidative phosphorylation (OXPHOS) in human adipose tissue ${ }^{20}$. Conflicting data regarding adipokine expression and secretion and lipolytic markers have been reported in human adipose tissue following exercise training ${ }^{21-29}$. 
Importantly, most human studies that have investigated the effect of exercise training on adipose tissue metabolism did not perform detailed metabolic phenotyping, and have not assessed the metabolic response to exercise training in parallel. Therefore, the aim of the present study was to investigate the effects of a 12 week supervised, progressive, combined exercise training program on abdominal subcutaneous adipose tissue gene expression of markers involved in mitochondrial biogenesis and function, browning, lipolysis, inflammation and adipokines in obese, well-phenotyped subjects. In addition, we compared the exercise training effects on abdominal subcutaneous adipose tissue gene expression between obese metabolically compromised (subjects with NAFL and T2DM) and obese metabolically healthy individuals.

\section{RESEARCH DESIGN AND METHODS}

\section{STUDY DESIGN}

Twenty-one sedentary, middle-aged (40-70 year), overweight/obese men (14 NAFL/T2DM and 7 control subjects) participated in the present study, which was a sub-study of a larger clinical trial designed to primarily investigate the effects of exercise training on liver fat content, hepatic, adipose tissue and peripheral insulin sensitivity (Brouwers B. et al., unpublished data). Control subjects $(n=7)$ were selected on having a liver fat content $\leq 4 \%$, as measured with proton magnetic resonance spectroscopy ( $\left.{ }^{1} \mathrm{H}-\mathrm{MRS}\right)$, in the absence of liver dysfunction (defined as alanine aminotransferase (ALAT) $>2.5 \times$ normal values) and had to be normoglycemic according to the WHO criteria. Subjects were defined as NAFL patients $(n=7)$ when having a liver fat content $\geq 5 \%$ as measured with MRS, in addition to a fasting plasma glucose concentration $<7.0 \mathrm{mmol} / \mathrm{l}$. Furthermore, T2DM patients ( $n=7)$ were allowed to be on sulphonyl urea or metformin therapy for at least 6 months with stable dosage for at least 2 months or on a dietary treatment for 6 months, with fasting plasma glucose concentrations $\geq 7.0 \mathrm{mmol} / \mathrm{I}$ and $<10.0 \mathrm{mmol} / \mathrm{l}$ at the time of screening. T2DM patients were not selected for liver fat content. Subjects were instructed not to change their habitual dietary intake during the study period. General exclusion criteria were unstable body 
weight, cardiovascular disease, impaired renal function, haemoglobin $<7.5 \mathrm{mmol} / \mathrm{l}$, blood pressure $>160 / 100 \mathrm{mmHg}$, participation in a weight loss or exercise training program, history of substantial alcohol use ( $>3$ units per day), history of drug abuse, use of beta-blockers, anti-thrombotic medication, insulin therapy and use of medication known to interfere with glucose homeostasis (except for T2DM patients).

At screening, routine laboratory analyses and physical examinations were performed, medical history was checked and a resting electrocardiogram (ECG) was taken. Maximal power output $\left(\mathrm{W}_{\max }\right)$ and maximal aerobic capacity $\left(\mathrm{VO}_{2 \max }\right)$ were assessed during a graded cycling test with concurrent ECG until exhaustion. Body composition was determined using DEXA (Hologic Discovery A, Waltham, MA, USA). All subjects gave written informed consent before participation in the study, which was approved by the Medical Ethical Committee of Maastricht University Medical Center +. All procedures were performed according the principles expressed in the Declaration of Helsinki.

\section{EXERCISE TRAINING PROTOCOL}

Subjects participated in a 12 week supervised, progressive exercise training program. Aerobic exercise training was carried out on a cycle ergometer twice a week for $30 \mathrm{~min}$ at $70 \%$ of $\mathrm{W}_{\max }$, which was determined just before the start of the intervention. In addition, resistance exercise training, which was focused on large muscle groups (chest press, lat pull down, leg extension, shoulder press, horizontal row, leg press, triceps extensions and biceps curls), was performed once a week and comprised three series of ten repetitions at $60 \%$ of subjects' previously determined one repeated maximum (1RM). The 1RM test was preceded by a familiarization trial. Warming-up and cooling-down sessions of $5 \mathrm{~min}$ were performed on a stationary bike at $45 \%$ of $\mathrm{W}_{\text {max }}$. Every 4 weeks, $1 \mathrm{RM}$ and maximal aerobic capacity were reassessed and training loads were adjusted accordingly to assure that the training stimulus was maintained. Supervised exercise training sessions were performed with 3-4 subjects simultaneously. At baseline and after the 12 week exercise training program, several measurements were performed, as described below. 


\section{ADIPOSE TISSUE BIOPSIES}

After an overnight fast, an abdominal subcutaneous adipose tissue biopsy ( $\sim 1 \mathrm{~g})$ was collected 6-8 cm lateral from the umbilicus, under local anaesthesia ( $2 \%$ lidocaine) by needle biopsy. The biopsy was washed with sterile saline, snap frozen in liquid nitrogen and stored at $-80^{\circ} \mathrm{C}$ until further analysis.

\section{ADIPOSE TISSUE GENE EXPRESSION}

Total RNA was extracted from frozen adipose tissue biopsies (500 mg) using Trizol chloroform extraction (Invitrogen, Cergy Pontoise, France) and 300 ng RNA was reversed transcribed using iscript cDNA synthesis kit (BIO-RAD). Gene expression for markers of lipolysis (ATGL [also known as PNPLA2], HSL [also known as LIPE], CGI-58 [also known as ABHD5], and PLIN1 [encoding perilipin 1]), inflammation (TNFa, IL-6, MCP-1 [also known as CCL2], CD68), browning (CIDEA, PRDM16), mitochondrial biogenesis (PGC-1 $\alpha$ [also known as PPARGC1A]) and adipokine expression (adiponectin and leptin) (for primer sequences see supplementary material) was determined in a total volume of $25 \mu \mathrm{L}$ containing $12.5 \mathrm{ng}$ CDNA using SYBR-Green based real-time PCRs (iCycler/MyIQ, BIO-RAD). Results were normalized for $18 \mathrm{~S}$ (housekeeping gene) ribosomal RNA.

\section{BIOCHEMISTRY}

Arterialized blood samples were collected and immediately centrifuged at $4{ }^{\circ} \mathrm{C}$ for $10 \mathrm{~min}$ at $1000 \mathrm{~g}$, and plasma was snap frozen in liquid nitrogen and stored at -80 ${ }^{\circ} \mathrm{C}$ until further analysis. Plasma free fatty acid (FFA; Wako Nefa C test kit; Wako Chemicals, Neuss, Germany) and glucose (FPG: hexokinase method; LaRoche, Basel, Switzerland) concentrations were measured with enzymatic assays, whereas triglyceride (TG) concentrations were measured colorimetrically (Roche, Vienna, Austria), automated on a Cobas Fara/Mira. Plasma insulin and serum liver function parameters (alanine transaminase (ALAT), aspartate aminotransferase (ASAT), $\gamma^{-}$ glutamyl transpeptidase (GGT)) were routinely measured and analyzed at the clinical chemistry department in the hospital. 


\section{STATISTICS}

Baseline comparisons between groups were analysed using Student's unpaired ttest. The effect of the 12 week exercise training intervention in the total group was assessed using Student's paired t-test. The effect of exercise training effects in obese NAFL/T2DM and obese metabolically healthy control subjects was compared by two-way repeated-measures ANOVA, using time (pre and post-intervention) as within-subject factor and group as between-subject factor. All variables were checked for normal distribution and were Ln-transformed to satisfy conditions of normality. Data are presented as means \pm SEM. Calculations were done using SPSS 21 for Mac OS X (IBM, Chicago, IL, USA). P $<0.05$ was considered statistically significant. 


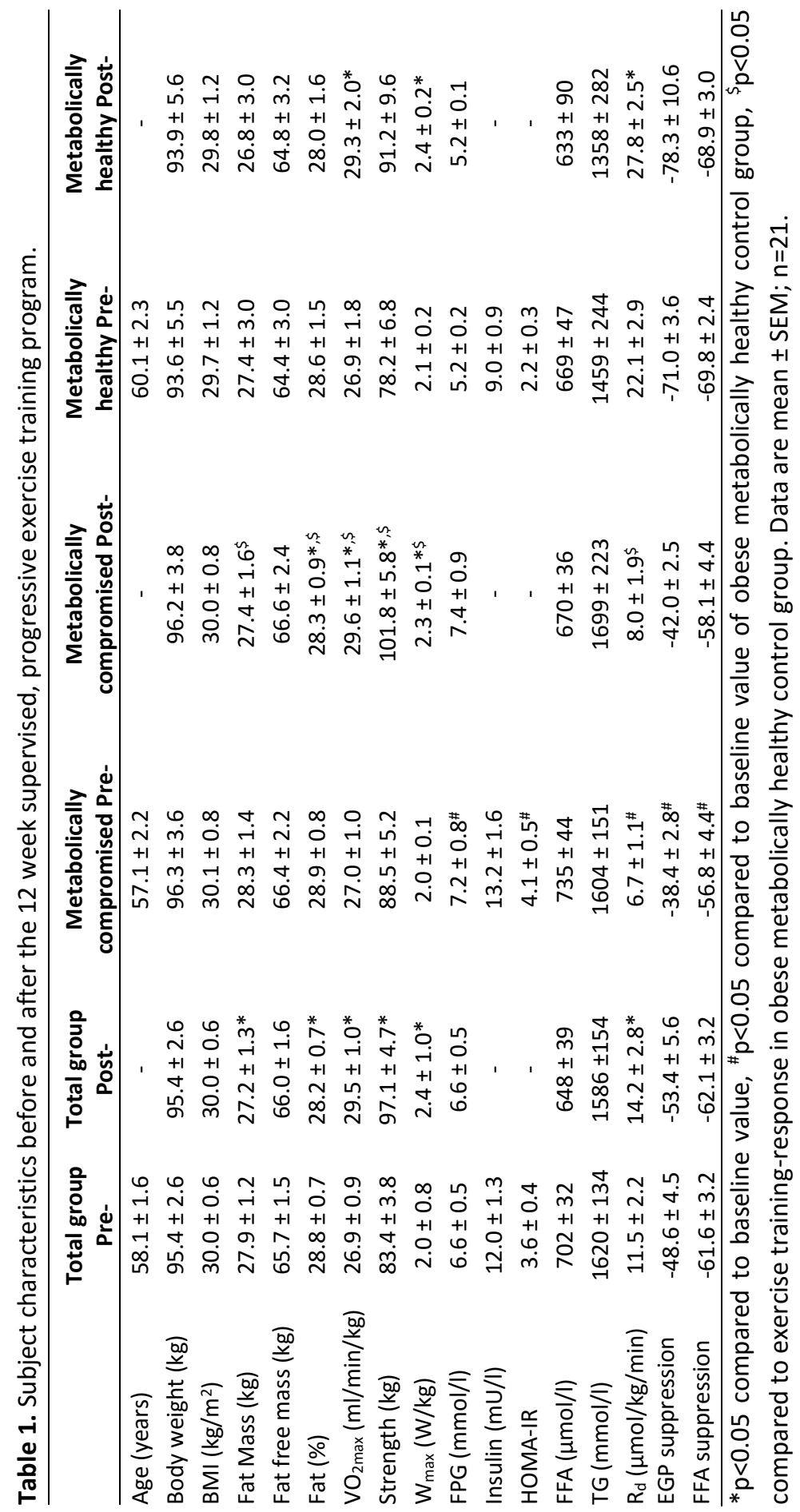




\section{RESULTS}

\section{ANTHROPOMETRIC AND CLINICAL CHARACTERISTICS}

Subject characteristics before and after the 12 week supervised, progressive exercise training program are summarized in table 1 . As previously observed in a larger cohort of subjects (Brouwers B. et al., unpublished data), fasting plasma glucose (FPG) and HOMA-IR were significantly higher at baseline in NAFL/T2DM subjects as compared to obese metabolically healthy controls, whereas peripheral, adipose tissue and hepatic insulin sensitivity were significantly lower in the metabolically compromised individuals (table 1). As expected, aerobic fitness and muscle strength were significantly increased after the exercise training intervention. Furthermore, total fat mass and body fat percentage were slightly but significantly decreased, whereas body weight, BMI, fat free mass and plasma metabolites were not significantly altered after the exercise training intervention (table 1).

\section{ADIPOSE TISSUE GENE EXPRESSION}

At baseline, there was a significantly lower gene expression of HSL $(0.35 \pm 0.08$ vs $0.45 \pm 0.11, p=0.005), C G I-58(0.39 \pm 0.04$ vs $0.75 \pm 0.19, p<0.001)$ and PGC- $1 \alpha$ ($0.35 \pm 0.22$ vs $0.00 \pm 0.13, p=0.037$ ) in the obese metabolically compromised as compared to obese metabolically healthy individuals. In the total group, adipose tissue gene expression of lipolysis remained unchanged following exercise training. More specific, gene expression of ATGL ( $p=0.253$, figure $1 a), H S L$ ( $p=0.875$, figure 1b), PLIN1 ( $p=0.733$, figure $1 c)$ and CGI-58 ( $p=0.966$, figure $1 d)$ were not affected by the intervention. Furthermore, the inflammatory markers TNF $\alpha(p=0.858$, figure 1e), IL6 ( $p=0.353$, figure 1f), MCP1 ( $p=0.144$, figure $1 g$ ) and CD68 ( $p=0.686$, figure $1 \mathrm{~h})$ were unchanged after the intervention. Next, the gene expression of browning markers CIDEA ( $p=0.901$, figure 11$)$ and PRDM16 $(p=0.872$, figure $1 j)$ and PGC- $1 \alpha$ $(p=0.793$, figure $1 k)$, which is a marker for mitochondrial biogenesis and function, were not affected by the intervention. Finally, gene expression of leptin ( $p=0.951$, figure $1 \mathrm{l}$ ) and adiponectin ( $p=0.340$, figure $1 \mathrm{~m}$ ) was not significantly altered by the 
12 week exercise training program. In line, no significant differences in exercise training induced alterations in these parameters were observed between groups, except for minor differential effects in CGI-58 ( $p=0.037)$ and TNF $\alpha(p=0.051)$.

\section{DISCUSSION}

The aim of the present study was to investigate the effect of a 12 week supervised, progressive exercise training program on abdominal subcutaneous adipose tissue gene expression in well-phenotyped obese individuals with or without NAFL/T2DM. Here, we demonstrate that a 12 week exercise training intervention did not significantly alter abdominal subcutaneous adipose tissue gene expression of markers for mitochondrial biogenesis and function, browning, lipolysis, inflammation and adipokines, irrespective of baseline metabolic status. In line with previous observations in a larger cohort (Brouwers B. et al., unpublished data), peripheral, hepatic and adipose tissue insulin sensitivity were significantly lower in obese metabolically compromised (NAFL/T2DM) as compared to obese metabolically healthy controls at baseline (table 1). The 12 week exercise training program significantly increased peripheral insulin sensitivity in the total group $(p=0.047)$, whereas no significant improvement in adipose tissue and hepatic insulin sensitivity were found, irrespective of metabolic status (table 1). These data suggest that alterations in the phenotype of abdominal subcutaneous adipose tissue do not substantially contribute to the previously reported exercise training induced improvement in peripheral insulin sensitivity in obese humans. The training program induced a significant increase in aerobic capacity $\left(\mathrm{VO}_{2 \max }\right)$, maximal muscle strength (1RM) and maximal power output $\left(\mathrm{W}_{\max }\right)$, indicating that the supervised, progressive nature of the program has been successful regarding enhancement of physical fitness. This was accompanied by a slight but significant decrease in fat mass and body fat percentage.

Since impaired lipolysis is one of the characteristics of adipose tissue dysfunction and relates to peripheral insulin resistance ${ }^{30,31}$, we determined adipose tissue gene expression of lipolytic enzymes and genes encoding lipid droplet-associated proteins, namely adipose triglyceride lipase (ATGL), hormone sensitive lipase (HSL), perilipin-1 (PLIN) and comparative gene identification-58 (CGI58). At baseline, a 
reduced expression of HSL and CGI-58 was found in obese metabolically compromised subjects as compared to obese metabolically healthy controls. These findings are in line with previous studies, showing a reduced expression of lipolytic genes in obese insulin resistant as compared to obese insulin sensitive individuals ${ }^{31}$ and patients with $\mathrm{T}_{2} \mathrm{DM}^{32}$. In the present study, exercise training did not affect the expression of these genes, irrespective of the metabolic phenotype (compromised or healthy) of the subjects.

In addition to impairments in lipid metabolism, low-grade inflammation in adipose tissue has been linked with insulin resistance in obese subjects and type 2 diabetic patients $s^{6-8}$. In the present study, no marked changes in adipose tissue gene expression of the pro-inflammatory factors TNF $\alpha$, IL- 6 and CD68 and the insulinsensitizing factor adiponectin were found following exercise training. These findings are in line with previous findings in obese subjects $22,23,25,26$, although a reduction in adipose tissue expression of MCP-1 has also been reported ${ }^{22}$, and increase in adiponectin mRNA expression ${ }^{23}$ have previously been reported after exercise training.

Rodent studies have demonstrated that increasing brown adipose tissue mass/activity or inducing browning of white adipose tissue, via cold or other factors, might be a promising strategy for the treatment of obesity and obesityrelated disorders such as $\mathrm{T}_{2} \mathrm{DM}^{33}$. Moreover, browning of white adipose tissue improved glucose homeostasis in mice ${ }^{34}$. Recently, cold acclimation and brown adipose tissue has also been shown to play a physiologically significant role in glucose homeostasis and insulin sensitivity in humans ${ }^{35}$. Interestingly, physical exercise has been linked to browning of white adipose tissue, which may be mediated via the secretion of the myokine irisin in mice ${ }^{14}$ or increased natriuretic peptide concentrations ${ }^{36}$. In accordance, the expression of browning markers in white adipose tissue of endurance-trained athletes was not different from lean sedentary controls ${ }^{37}$. In the present study, the 12 week exercise training program did not change the expression of two major brown fat-selective genes (CIDEA and PRDM16). In agreement with our findings, no increase in browning markers was found after 10 consecutive days of exercise in healthy lean subjects ${ }^{38}$, after a 12 week exercise training program in lean and overweight men $^{39}$, and following a 6 month exercise training in healthy overweight subjects ${ }^{20}$. Thus, our data, together 
with previous findings, suggests that exercise training does not induce browning of abdominal subcutaneous adipose tissue in humans.
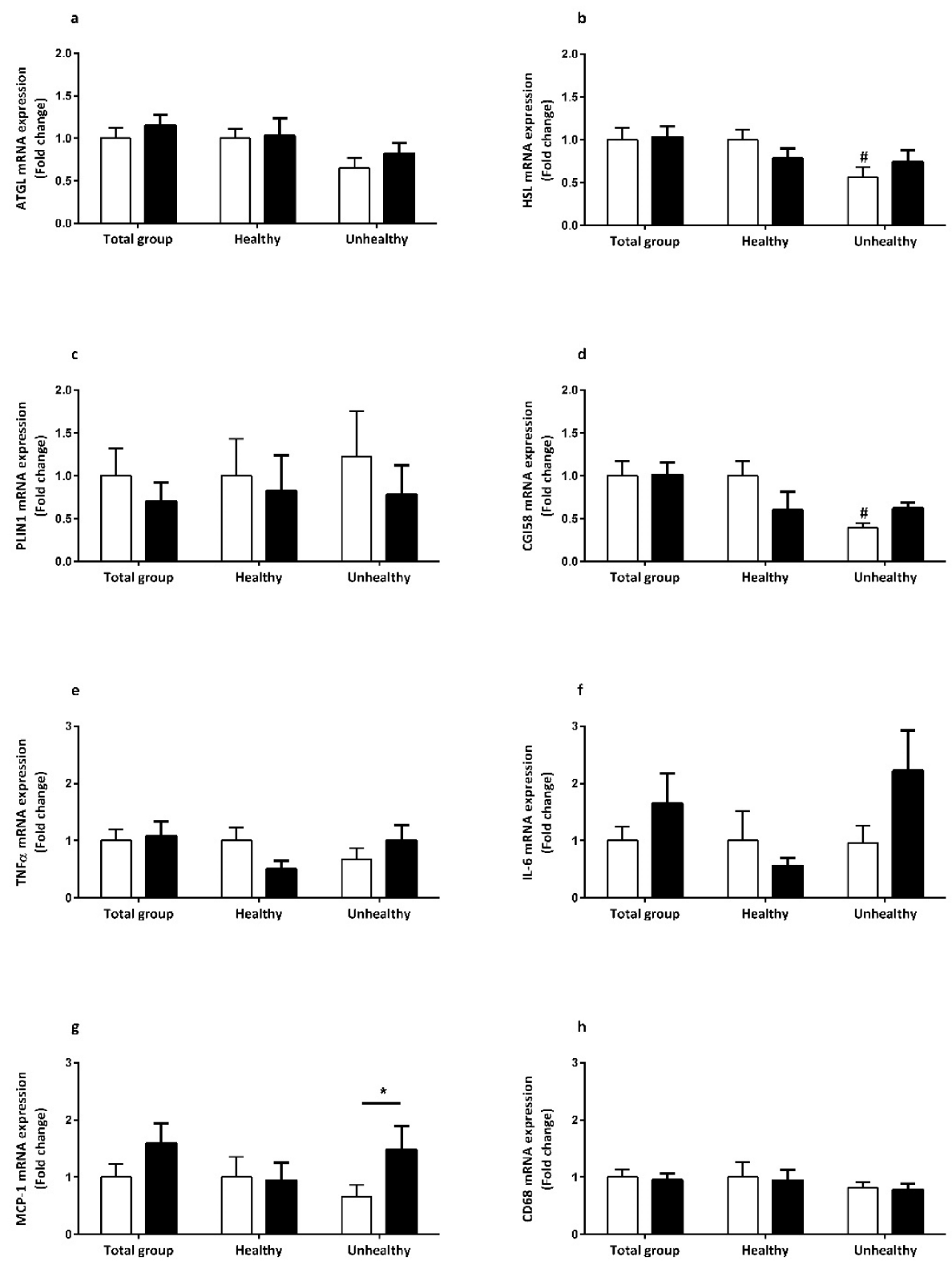

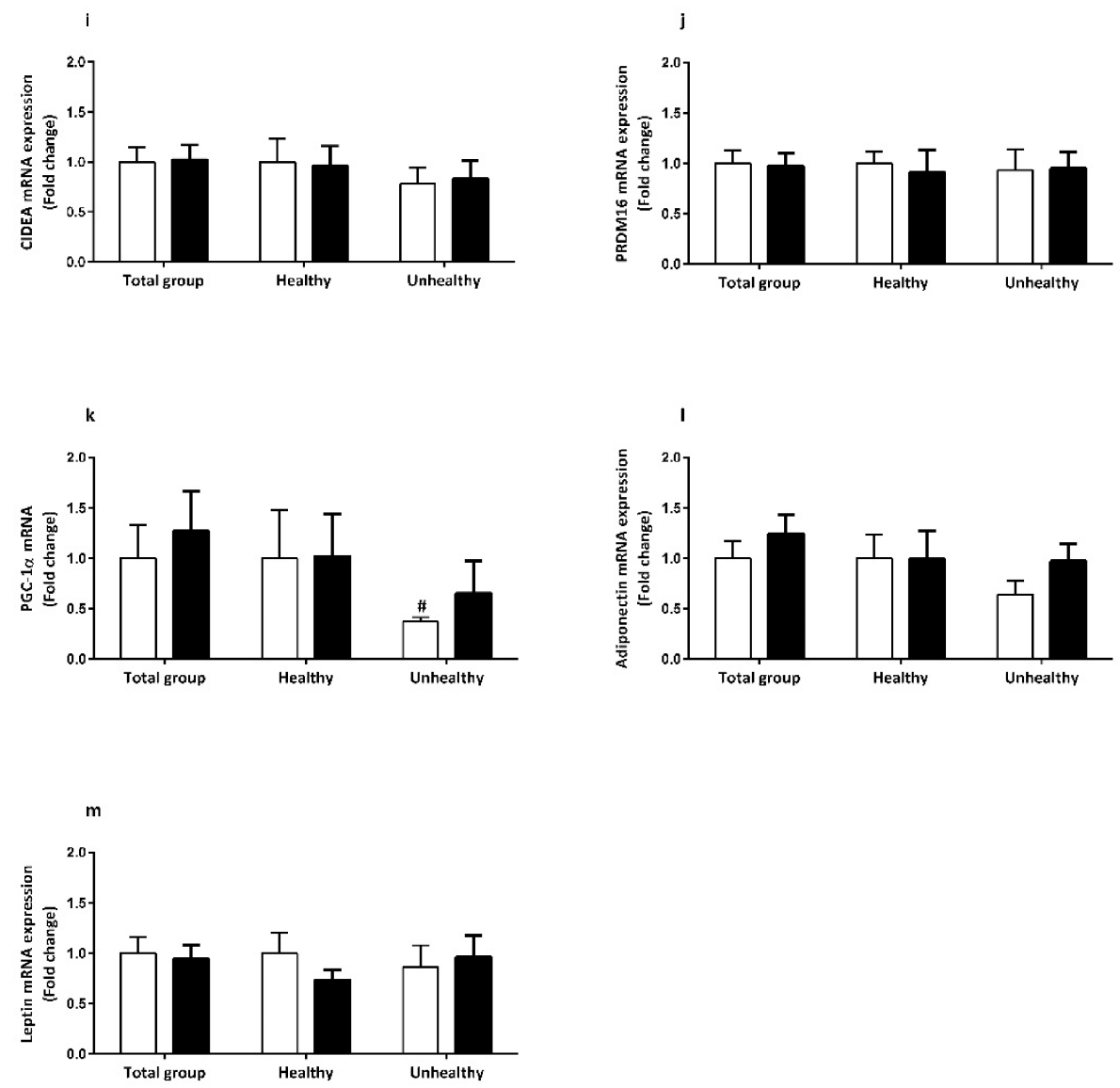

Figure 1. Exercise training-induced effects on gene expression in abdominal subcutaneous adipose tissue. Relative expression of genes involved in lipolysis (a-d), inflammation (e-h), browning (i-j), mitochondrial biogenesis and function (k), and adipokine expression (l-m) is shown. ${ }^{*} p<0.05$ compared to pre-exercise. ${ }^{*} p<0.05$ compared to baseline value of obese metabolically healthy control group. White columns, baseline values; black columns, postintervention values. Data are mean \pm SEM.

The oxidative phenotype of adipose tissue is associated with insulin resistance and T2DM in rodents ${ }^{40}$. Interestingly, we found that baseline gene expression of PGC$1 \alpha$, a key regulator of mitochondrial function and biogenesis, was significantly lower in obese metabolically compromised as compared to obese controls. In accordance with this, reduced expression of PGC-1 $\alpha$ has previously been shown in subcutaneous adipose tissue of obese, insulin resistant and T2DM patients ${ }^{19,41-43}$. 
Furthermore, an unsupervised informatics analysis using $>1000$ human adipose tissue biopsies has shown that mitochondrial oxidative pathways were markedly downregulated in morbidly obese insulin resistant as compared to morbidly obese insulin sensitive patients ${ }^{44}$. In the present study, we were not able to demonstrate alterations in adipose tissue gene expression of PGC-1 $\alpha$. This is in accordance with previous findings ${ }^{20,38,45}$, although data seem controversial ${ }^{19,43}$.

Finally, we assessed the effects of exercise training on the expression of leptin and adiponectin, which are involved in glucose homeostasis ${ }^{6,7}$. The present study indicated that exercise training did not affect leptin and adiponectin gene expression in adipose tissue. Although some studies have found increased adipose tissue leptin ${ }^{22}$ and adiponectin ${ }^{24}$ gene expression in overweight/obese subjects, several studies have demonstrated that leptin and adiponectin expression remained unchanged after exercise training in both lean and overweight/obese humans $s^{25,26,45}$.

In conclusion, the present study demonstrated that a supervised, progressive 12 week exercise training intervention, which improved physical fitness and peripheral insulin sensitivity, had no significant effects on abdominal subcutaneous adipose tissue gene expression of markers of lipolysis, inflammation, mitochondrial biogenesis and function, browning of white adipose tissue and adipokine expression in obese individuals, irrespective of baseline metabolic status. Thus, exercise training mediated improvements in peripheral insulin sensitivity in healthy and metabolically compromised obese subjects does not require adaptive responses in markers of subcutaneous white adipose tissue function. Noteworthy, we cannot exclude that exercise training may induce beneficial alterations in other fat depots, such as visceral adipose tissue, or after a more prolonged period. 


\section{SUPPLEMENTARY MATERIAL}

The following Real-Time RT-PCR Primer Sequences were used for gene expression in white adipose tissue.

\begin{tabular}{|c|c|}
\hline Genes & Sequences \\
\hline \multirow[t]{2}{*}{ ATGL } & GTGTCAGACGGCGAGAATG \\
\hline & TGGAGGGAGGGAGGGATG \\
\hline \multirow[t]{2}{*}{ HSL } & GCGGATCACACAGAACCTGGAC \\
\hline & AGCAGGCGGCTTACCCTCAC \\
\hline \multirow[t]{2}{*}{ CGI-58 } & CAGCATCCAGTCCTTACGACCA \\
\hline & GTTCAGTCCACAGTGTCGCAGA \\
\hline \multirow[t]{2}{*}{ PLIN1 } & CTCTCGATACACCGTGCAGA \\
\hline & TGGTCCTCATGATCCTCCTC \\
\hline \multirow[t]{2}{*}{ TNF $\alpha$} & CCGAGTGACAAGCCTGTAGC \\
\hline & GAGGACCTGGGAGTAGATGAG \\
\hline \multirow[t]{2}{*}{ IL-6 } & AAATTCGGTACATCCTCGACGG \\
\hline & GGAAGGTTCAGGTTGTTTTCTGC \\
\hline \multirow[t]{2}{*}{ MCP-1 } & CCCCAGTCACCTGCTGTTAT \\
\hline & TCCTGAACCCACTTCTGCTT \\
\hline \multirow[t]{2}{*}{ CD68 } & СCCTATGGACACCTCAGCTTT \\
\hline & GAAGGACACATTGTACTCCACC \\
\hline \multirow[t]{2}{*}{ CIDEA } & TCAGACCTTGGGAGACAACACG \\
\hline & CGAAGGTGACTCTCGCTATTCC \\
\hline \multirow[t]{2}{*}{ PRDM16 } & CAGCCAATCTCACCAGACACCT \\
\hline & GTGGCACTTGAAAGGCTTCTCC \\
\hline \multirow[t]{2}{*}{ PGC-1 $\alpha$} & TCTGAGTCTGTATGGAGTGACAT \\
\hline & CCAAGTCGTTCACATCTAGTTCA \\
\hline
\end{tabular}




\section{REFERENCES}

1. Kopelman, P.G. Obesity as a medical problem. Nature 404, 635-643 (2000).

2. Mann, S., et al. Changes in insulin sensitivity in response to different modalities of exercise: a review of the evidence. Diabetes/metabolism research and reviews 30, 257-268 (2014).

3. Roberts, C.K., Little, J.P. \& Thyfault, J.P. Modification of insulin sensitivity and glycemic control by activity and exercise. Medicine and science in sports and exercise 45, 1868-1877 (2013).

4. Meex, R.C., et al. Restoration of muscle mitochondrial function and metabolic flexibility in type 2 diabetes by exercise training is paralleled by increased myocellular fat storage and improved insulin sensitivity. Diabetes 59, 572-579 (2010).

5. Colberg, S.R., et al. Exercise and type 2 diabetes: the American College of Sports Medicine and the American Diabetes Association: joint position statement. Diabetes care 33, e147-167 (2010).

6. Goossens, G.H. The role of adipose tissue dysfunction in the pathogenesis of obesity-related insulin resistance. Physiology \& behavior 94, 206-218 (2008).

7. Rosen, E.D. \& Spiegelman, B.M. What we talk about when we talk about fat. Cell 156, 20-44 (2014).

8. Stinkens, R., Goossens, G.H., Jocken, J.W. \& Blaak, E.E. Targeting fatty acid metabolism to improve glucose metabolism. Obesity reviews : an official journal of the International Association for the Study of Obesity 16, 715-757 (2015).

9. Sutherland, L.N., Bomhof, M.R., Capozzi, L.C., Basaraba, S.A. \& Wright, D.C. Exercise and adrenaline increase PGC-1\{alpha\} mRNA expression in rat adipose tissue. The Journal of physiology 587, 1607-1617 (2009).

10. Trevellin, E., et al. Exercise training induces mitochondrial biogenesis and glucose uptake in subcutaneous adipose tissue through eNOS-dependent mechanisms. Diabetes 63, 2800-2811 (2014).

11. Stallknecht, B., Vinten, J., Ploug, T. \& Galbo, H. Increased activities of mitochondrial enzymes in white adipose tissue in trained rats. The American journal of physiology 261, E410-414 (1991). 
12. Stanford, K.I., et al. A novel role for subcutaneous adipose tissue in exercise-induced improvements in glucose homeostasis. Diabetes 64, 2002-2014 (2015).

13. Vernochet, C., et al. Adipose-specific deletion of TFAM increases mitochondrial oxidation and protects mice against obesity and insulin resistance. Cell metabolism 16, 765-776 (2012).

14. Bostrom, P., et al. A PGC1-alpha-dependent myokine that drives brown-fatlike development of white fat and thermogenesis. Nature 481, 463-468 (2012).

15. Cao, L., et al. White to brown fat phenotypic switch induced by genetic and environmental activation of a hypothalamic-adipocyte axis. Cell metabolism 14, 324-338 (2011).

16. Zachwieja, J.J., Hendry, S.L., Smith, S.R. \& Harris, R.B. Voluntary wheel running decreases adipose tissue mass and expression of leptin mRNA in Osborne-Mendel rats. Diabetes 46, 1159-1166 (1997).

17. Bradley, R.L., Jeon, J.Y., Liu, F.F. \& Maratos-Flier, E. Voluntary exercise improves insulin sensitivity and adipose tissue inflammation in dietinduced obese mice. American journal of physiology. Endocrinology and metabolism 295, E586-594 (2008).

18. Stanford, K.I., Middelbeek, R.J. \& Goodyear, L.J. Exercise Effects on White Adipose Tissue: Beiging and Metabolic Adaptations. Diabetes (2015).

19. Ruschke, K., et al. Gene expression of PPARgamma and PGC-1alpha in human omental and subcutaneous adipose tissues is related to insulin resistance markers and mediates beneficial effects of physical training. European journal of endocrinology / European Federation of Endocrine Societies 162, 515-523 (2010).

20. Ronn, T., et al. Extensive changes in the transcriptional profile of human adipose tissue including genes involved in oxidative phosphorylation after a 6-month exercise intervention. Acta physiologica 211, 188-200 (2014).

21. Bluher, M., et al. Gene expression of adiponectin receptors in human visceral and subcutaneous adipose tissue is related to insulin resistance and metabolic parameters and is altered in response to physical training. Diabetes care 30, 3110-3115 (2007). 
22. Sjogren, P., et al. Functional changes in adipose tissue in a randomised controlled trial of physical activity. Lipids in health and disease 11, 80 (2012).

23. Trachta, P., et al. Three months of regular aerobic exercise in patients with obesity improve systemic subclinical inflammation without major influence on blood pressure and endocrine production of subcutaneous fat. Physiological research / Academia Scientiarum Bohemoslovaca 63 Suppl 2, S299-308 (2014).

24. Moghadasi, M., et al. High-intensity endurance training improves adiponectin mRNA and plasma concentrations. European journal of applied physiology 112, 1207-1214 (2012).

25. Klimcakova, E., et al. Dynamic strength training improves insulin sensitivity without altering plasma levels and gene expression of adipokines in subcutaneous adipose tissue in obese men. The Journal of clinical endocrinology and metabolism 91, 5107-5112 (2006).

26. Polak, J., et al. Effect of aerobic training on plasma levels and subcutaneous abdominal adipose tissue gene expression of adiponectin, leptin, interleukin 6, and tumor necrosis factor alpha in obese women. Metabolism: clinical and experimental 55, 1375-1381 (2006).

27. Hulver, M.W., et al. Adiponectin is not altered with exercise training despite enhanced insulin action. American journal of physiology. Endocrinology and metabolism 283, E861-865 (2002).

28. Richterova, B., et al. Effect of endurance training on adrenergic control of lipolysis in adipose tissue of obese women. The Journal of clinical endocrinology and metabolism 89, 1325-1331 (2004).

29. Covington, J.D., et al. Potential effects of aerobic exercise on the expression of perilipin 3 in the adipose tissue of women with polycystic ovary syndrome: a pilot study. European journal of endocrinology / European Federation of Endocrine Societies 172, 47-58 (2015).

30. Girousse, A., et al. Partial inhibition of adipose tissue lipolysis improves glucose metabolism and insulin sensitivity without alteration of fat mass. PLoS biology 11, e1001485 (2013).

31. Jocken, J.W., et al. Adipose triglyceride lipase and hormone-sensitive lipase protein expression is decreased in the obese insulin-resistant state. The Journal of clinical endocrinology and metabolism 92, 2292-2299 (2007). 
32. Watt, M.J., et al. Hormone-sensitive lipase is reduced in the adipose tissue of patients with type 2 diabetes mellitus: influence of IL- 6 infusion. Diabetologia 48, 105-112 (2005).

33. Harms, M. \& Seale, P. Brown and beige fat: development, function and therapeutic potential. Nature medicine 19, 1252-1263 (2013).

34. Mossenbock, K., et al. Browning of white adipose tissue uncouples glucose uptake from insulin signaling. PloS one 9, e110428 (2014).

35. Hanssen, M.J., et al. Short-term cold acclimation improves insulin sensitivity in patients with type 2 diabetes mellitus. Nature medicine 21, 863-865 (2015).

36. Bordicchia, M., et al. Cardiac natriuretic peptides act via p38 MAPK to induce the brown fat thermogenic program in mouse and human adipocytes. The Journal of clinical investigation 122, 1022-1036 (2012).

37. Vosselman, M.J., et al. Low brown adipose tissue activity in endurancetrained compared with lean sedentary men. International journal of obesity (2015).

38. Camera, D.M., Anderson, M.J., Hawley, J.A. \& Carey, A.L. Short-term endurance training does not alter the oxidative capacity of human subcutaneous adipose tissue. European journal of applied physiology 109, 307-316 (2010).

39. Norheim, F., et al. The effects of acute and chronic exercise on PGC-1alpha, irisin and browning of subcutaneous adipose tissue in humans. The FEBS journal 281, 739-749 (2014).

40. Kusminski, C.M. \& Scherer, P.E. Mitochondrial dysfunction in white adipose tissue. Trends Endocrinol Metab 23, 435-443 (2012).

41. Hammarstedt, A., Jansson, P.A., Wesslau, C., Yang, X. \& Smith, U. Reduced expression of PGC-1 and insulin-signaling molecules in adipose tissue is associated with insulin resistance. Biochemical and biophysical research communications 301, 578-582 (2003).

42. Semple, R.K., et al. Expression of the thermogenic nuclear hormone receptor coactivator PGC-1alpha is reduced in the adipose tissue of morbidly obese subjects. International journal of obesity and related metabolic disorders : journal of the International Association for the Study of Obesity 28, 176-179 (2004). 
43. Khadir, A., et al. MAP kinase phosphatase DUSP1 is overexpressed in obese humans and modulated by physical exercise. American journal of physiology. Endocrinology and metabolism 308, E71-83 (2015).

44. Qatanani, M., et al. Inverse regulation of inflammation and mitochondrial function in adipose tissue defines extreme insulin sensitivity in morbidly obese patients. Diabetes 62, 855-863 (2013).

45. Alvehus, M., Boman, N., Soderlund, K., Svensson, M.B. \& Buren, J. Metabolic adaptations in skeletal muscle, adipose tissue, and whole-body oxidative capacity in response to resistance training. European journal of applied physiology 114, 1463-1471 (2014). 




\section{CHAPTER 7}

\section{COMBINED AEROBIC AND RESISTANCE}

EXERCISE TRAINING IMPROVES THE CARDIOMETABOLIC PROFILE IN OVERWEIGHT AND OBESE MEN WITH METABOLIC SYNDROME

Bram Brouwers, Peter J. Joris, Matthijs K.C. Hesselink, Patrick Schrauwen, Vera B. Schrauwen-Hinderling

In preparation for submission 


\section{ABSTRACT}

Overweight and obesity is associated with increased risk for diabetes, especially when fasted glucose is also elevated. In this population at risk for metabolic disease, also incidence of cardiovascular disease is high. To define a subclass with elevated cardiovascular risk, the metabolic syndrome (MetS) was defined, considering also several markers of cardiovascular risk. Physical activity prevents and treats obesityrelated diseases and next to dietary changes, increase in physical activity is advocated. We examined whether combined aerobic and resistance exercise training (CT) improved the metabolic profile in overweight and slightly obese men with MetS. We were interested to address which risk components of the MetS were affected by CT. Furthermore, we performed more detailed measurements to assess cardiovascular risk.

Twelve overweight/obese (BMI $27-35 \mathrm{~kg} / \mathrm{m}^{2}$ ), middle-aged male subjects (age 40 - 70 years old) with MetS participated in a 12 week progressive CT program. Body composition, maximal aerobic capacity $\left(\mathrm{VO}_{2 \max }\right)$, MetS-score and individual risk components (waist circumference (WC), triglycerides (TG), high density lipoprotein cholesterol (HDL-C), blood pressure and fasting plasma glucose (FPG)) were measured before and after $\mathrm{CT}$. Pulse wave analysis to derive central augmentation index (Alx), subendocardial viability ratio (SEVR) and central pressure, and echocardiography to measure cardiac function were performed pre- and post-CT. CT significantly improved $\mathrm{VO}_{2 \max }(\mathrm{p}<0.001)$ in people with MetS. Average MetSscore significantly dropped from $3.5 \pm 0.2$ to $2.4 \pm 0.3$ ( $p=0.003$ ), and seven subjects were cured from having the MetS. CT did not significantly change WC, TG, HDL-C, diastolic blood pressure and FPG. CT significantly reduced systolic blood pressure (from $141.1 \pm 4.7$ to $130.5 \pm 3.6 \mathrm{mmHg}, p=0.004$ ), pulse pressure (from $52.3 \pm 3.9$ to $46.0 \pm 2.0 \mathrm{mmHg}, p=0.031$ ), and central systolic blood pressure (from $129.8 \pm 5.7$ to $121.0 \pm 4.7 \mathrm{mmHg}, p=0.043)$. Alx $(p=0.566)$, SEVR $(p=0.579)$ and parameters for cardiac function were unaffected by CT.

In conclusion, CT improves the cardio-metabolic profile of overweight and obese men with MetS. CT formally reduced the number of people with MetS as hypertension, one of the components contributing to MetS, did no longer exceed the cut-off level. Despite the positive effect on the cardio-metabolic profile, CT does not improve cardiac function and arterial stiffness. 


\section{INTRODUCTION}

Over the last 25 years, there has been a steady increase in BMI in the general population ${ }^{1}$. This increases the risk for diabetes, certainly in the older age groups and when fasting plasma glucose (FPG) is elevated. To also detect increased cardiovascular risk, the metabolic syndrome (MetS) was defined as a constellation of cardiovascular risk factors ${ }^{2}$. According to the International Diabetes Federation (IDF) definition, the key components of the MetS include central obesity, dyslipidemia (elevated levels of plasma triglycerides (TG), decreased levels of high density lipoprotein cholesterol (HDL-C)), hypertension and hyperglycemia ${ }^{3}$. Approximately $25 \%$ of the global adult population has the metabolic syndrome $e^{4,5}$. Among men, nearly $30 \%$ of those who are overweight and $65 \%$ of those who are obese meet the criteria for the MetS ${ }^{6}$.

Management of the MetS includes recommendation of regular physical exercise ${ }^{7}$. In current American Diabetes Association guidelines, the target is $150 \mathrm{~min}$ of moderate-intensity exercise per week to prevent and treat obesity-related diseases ${ }^{8}$. Exercise training is also an effective way to improve cardiac function and cardiovascular risk ${ }^{9}$. Numerous studies have shown that combining diet with exercise training is very effective in the prevention of MetS ${ }^{10}$. However, increasing physical activity, combined with targeted weight loss is difficult and therefore adherence is low, especially on the longer term. Relative few studies have investigated the effect of exercise training on the MetS per $s e^{10}$, but most have demonstrated that exercise decreases the metabolic risk of people with MetS ${ }^{11-14}$. Most studies have focused on the effects of aerobic exercise training. Aerobic exercise training aims to increase endurance performance, leading to increases in maximal aerobic capacity and cardiac output ${ }^{15}$. Resistance exercise training increases muscular strength and induces muscle hypertrophy, leading to a higher resting metabolic rate ${ }^{15,16}$. Moreover, increased muscle strength and mass may ameliorate the effect of aerobic exercise training. Therefore, adding resistance exercise to aerobic exercise is known to increase the beneficial effects of exercise training on cardio-metabolic parameters ${ }^{16}$.

Central pressures and indices have a high predictability for the pathogenesis of cardiovascular events ${ }^{17-19}$. Enhanced augmentation of the pressure waveform is a 
manifestation of vascular aging and arterial stiffness, and is directly related to cardiovascular risk ${ }^{20}$. The augmentation index (Alx) is a non-invasive measure of enhanced augmentation and a lower Alx is associated with a lower risk of cardiovascular disease ${ }^{21}$. In a meta-analysis ${ }^{21}$ that included 11 longitudinal studies with a mean follow-up of 45 months, central pressures and measures of arterial stiffness, such as Alx, had significant predictive value for future cardiovascular events. Furthermore, Alx had significant predictive value for all-cause mortality, independent of blood pressure and heart rate. Because these parameters can be reliably assessed non-invasively with relatively inexpensive devices, they are very useful for clinical practice.

The purpose of the present study was to investigate whether combined aerobic and resistance exercise training (CT) is an effective tool to improve the cardio-metabolic profile in overweight and obese men with the MetS. To this end, we measured which traditional risk components of the MetS were affected by CT. We also measured Alx, central pressure, SEVR and echocardiography to address the effect of $\mathrm{CT}$ on vascular and cardiac function.

\section{RESEARCH DESIGN AND METHODS}

Twelve Caucasian overweight and slightly obese men with MetS were included in this study. Subjects with MetS were selected from a larger cohort of overweight/obese individuals who participated in a larger clinical trial designed to primarily investigate the effects of exercise training on liver fat content and insulin sensitivity (Brouwers B. et al. ${ }^{22}$, unpublished data). Subjects were sedentary, overweight/obese (BMI $27-35 \mathrm{~kg} / \mathrm{m}^{2}$ ), middle-aged ( $40-70$ years old) with stable dietary habits. General exclusion criteria were unstable body weight (weight gain or loss $>3 \mathrm{~kg}$ in the past 3 months), cardiac disease, diabetes, use of beta-blockers, use of anti-thrombotic medication, elevated blood pressure ( $>160 / 100 \mathrm{mmHg}$ ), history of substantial alcohol use ( $>3$ units per day), history of drug abuse and participation in a weight loss or vigorous exercise program. Before the onset of the study, routine medical laboratory, a medical history and physical examination was performed, and a resting electrocardiogram was taken. Maximal work load ( $\left.\mathrm{W}_{\max }\right)$ and maximal aerobic capacity $\left(\mathrm{VO}_{2 \max }\right)$ were assessed during a graded cycling test 
with concurrent ECG until exhaustion ${ }^{23}$. All subjects gave written informed consent before participation in the study. The Maastricht University Medical Ethical Committee approved the study. The study was performed according the principles expressed in the most recent version of the declaration of Helsinki.

\section{EXERCISE TRAINING PROTOCOL}

Subjects enrolled in a tightly controlled combined aerobic and resistance exercise training program for 12 weeks. Aerobic exercise was carried out on a cycling ergometer twice a week for $30 \mathrm{~min}$ at $70 \%$ of the previously determined $\mathrm{W}_{\max }$. Resistance exercise was performed once a week and comprised three series of ten repetitions at $60 \%$ of subjects' previously determined maximal voluntary contractions (1RM) and focused on large muscle groups (chest press, lat pull down, leg extension, shoulder press, horizontal row, leg press, triceps curls and biceps curls). The $1 \mathrm{RM}$ test was preceded by a familiarization trial. Warming-up and cooling-down sessions of $5 \mathrm{~min}$ before aerobic and resistance exercise were performed on a stationary bike at $45 \%$ of $\mathrm{W}_{\max }$. Every 4 weeks, $1 \mathrm{RM}$ and $\mathrm{VO}_{2 \max }$ were reassessed, and training loads were readjusted accordingly. Supervised training sessions were performed with 3-4 subjects at a time.

\section{RISK COMPONENTS OF METS AND THE METS-SCORE}

MetS was defined by the presence of central obesity (waist circumference (WC) > $94 \mathrm{~cm}$ in men) plus any 2 of the following 4 metabolic risk components as defined by the IDF ${ }^{3}$ : (1) Systolic blood pressure (SBP) $\geq 130 \mathrm{mmHg}$ or diastolic blood pressure (DBP) $\geq 85 \mathrm{mmHg}$; (2) HDL-C $<1.03 \mathrm{mmol} / \mathrm{l}$; (3) FPG $<5.6 \mathrm{mmol} / \mathrm{l}$; and (4) $\mathrm{TG} \geq 1.7 \mathrm{mmol} / \mathrm{l}$. To measure the overall effects of exercise training, the MetS-score and the risk components of the MetS were examined. The MetS-score is a clinically useful index of MetS severity that is correlated with atherosclerotic risk factors and the severity of $C V D^{24}$. The value of the MetS-score was calculated as the sum of each participant's risk components of the MetS (WC, plasma TG, inverted HDL-C, $\mathrm{SBP}, \mathrm{DBP}$, and FPG ${ }^{25}$. The MetS-score ranged from 0 (no risk components of MetS) to 5 (presence of all 5 risk components of MetS). 


\section{ANTHROPOMETRIC ASSESSMENT AND BLOOD PRESSURE}

WC was assessed at the end of exhalation, with the subjects standing erect and relaxed with arms in the sides and feet positioned close together. The WC was measured to the nearest $0.1 \mathrm{~cm}$ in a horizontal plane around the abdomen at the level of the iliac crest. Blood pressure was measured using a semi-continuous blood-pressure monitor (Omron M7; Cemex Medische Techniek BV, Nieuwegein, The Netherlands). After a rest period of $30 \mathrm{~min}$, participants were fitted with an appropriately sized cuff in supine position and blood pressure was assessed 3 times, with the first measurement discarded and the average of the last 2 measurements reported. WC, blood pressure (SBP and DBP) and heart rate were determined 2 days before and 2 days after the $C T$ intervention. Pulse pressure was calculated as the difference between SBP and DBP.

\section{PULSE WAVE ANALYSIS}

Vascular measurements were performed in a quiet, darkened room in 11 subjects. The room was temperature controlled at $24{ }^{\circ} \mathrm{C}$. Radial artery pulse wave analysis (PWA) was performed with a non-invasive tonometer (SphygmoCor v9; AtCor Medical, West Ryde, Australia) applied to the radial artery near the wrist of the arm. The central waveform was derived from the peripheral waveform using a generalized transfer function ${ }^{26}$. Central augmentation indices (Alx) were defined as the difference between the first and second peak of the arterial waveform, expressed as a percentage of the pulse pressure and corrected for heart rate. The myocardial perfusion relative to left ventricle workload has been indirectly estimated by subendocardial viability ratio (SEVR), and is calculated as previously described ${ }^{27}$. A critical value for SEVR of 0.5 has been suggested ${ }^{27}$, below which insufficient subendocardial perfusion may occur. 


\section{ECHOCARDIOGRAPHY}

Cardiac function was estimated using ultrasound before and after CT. M-mode, two-dimensional and Doppler echocardiography was performed, using a Vivid 7 ultrasound system (GE healthcare, Milwaukee, WI, USA) with $3.5 \mathrm{MHz}$ cardiac transducer. E/A ratio, left ventricular ejection fraction (LVEF), left ventricular enddiastolic diameter (LVEDD) and left ventricular end-systolic diameter (LVESD) were used as markers for the function of the left ventricle of the heart.

\section{BLOOD SAMPLING}

Arterialized venous blood samples were collected from an antecubital vein in NaFcontaining/serum tubes (Becton, Dickinson and Company, Franklin Lanes, NY, USA) and immediately centrifuged at high speed (speed/time for serum/plasma samples), and plasma was frozen in liquid nitrogen and stored at $-80{ }^{\circ} \mathrm{C}$ until analysis at the end of the study. FPG was measured with enzymatic assays automated on a Cobas Fara/Mira (hexokinase method; LaRoche, Basel, Switzerland). Plasma TG were measured colorimetrically (Roche, Vienna, Austria). Total cholesterol (Roche Diagnostics, Mannheim, Germany) and HDL-C (Roche Diagnostics, Mannheim, Germany), after precipitation of apolipoprotein B containing lipoproteins with phosphotungstic acid and magnesium ions, were analyzed in serum enzymatically (GPO-Trinder; Sigma-Aldrich Corp., St. Louis, MO, USA). Low density lipoprotein cholesterol (LDL-C) was calculated according to the Friedewald equation ${ }^{28}$, with no subjects having plasma TG levels above $4.5 \mathrm{mmol} / \mathrm{l}$.

\section{STATISTICAL ANALYSES}

All values are reported as the mean \pm standard error of the mean (SEM). Statistical analysis was performed using IBM SPSS 21 (SPSS Incorporated, Chicago, IL, USA). Differences were considered statistically significant at $P<0.05$. A paired-samples $t-$ test was used to determine whether there was a statistically significant mean difference between pre- and post-intervention values. 


\section{RESULTS}

\section{BASELINE CHARACTERISTICS}

Subjects were $55.3 \pm 2.1$ years old and had a BMl of $30.4 \pm 0.9 \mathrm{~kg} / \mathrm{m}^{2}$. Average MetSscore pre-exercise was $3.5 \pm 0.2$ per subject. All subjects had WC above $94 \mathrm{~cm}$ and in 6 subjects plasma TG levels exceeded $1.7 \mathrm{mmol} / \mathrm{I}$. Six subjects had HDL-C concentration lower than $1.03 \mathrm{mmol} / \mathrm{l}$. All participants were characterized with SBP $>130 \mathrm{mmHg}$ or DBP $>85 \mathrm{mmHg}$, whereas seven individuals were diagnosed with FPG concentration exceeding the $5.6 \mathrm{mmol} / \mathrm{l}$ cut-off value.

Table 1. Pre- and post-CT values in people with MetS.

\begin{tabular}{lccc}
\hline & Pre-CT & Post-CT & P value \\
\hline BMI $\left(\mathrm{kg} / \mathrm{m}^{2}\right)$ & $30.4 \pm 0.9$ & $30.1 \pm 0.9$ & 0.159 \\
$\mathrm{VO}_{2 \mathrm{max}}(\mathrm{ml} / \mathrm{min} / \mathrm{kg})$ & $25.9 \pm 1.3$ & $29.3 \pm 1.5$ & $<0.001$ \\
$\mathrm{~W}_{\max }(\mathrm{W} / \mathrm{kg})$ & $2.0 \pm 0.1$ & $2.4 \pm 0.1$ & $<0.001$ \\
MetS-score & $3.5 \pm 0.2$ & $2.4 \pm 0.3$ & 0.003 \\
Waist circumference (cm) & $107.9 \pm 2.2$ & $107.3 \pm 2.3$ & 0.159 \\
Triglycerides (mmol/l) & $1.55 \pm 0.20$ & $1.61 \pm 0.27$ & 0.761 \\
HDL-C (mmol/l) & $1.07 \pm 0.05$ & $1.14 \pm 0.07$ & 0.155 \\
SBP (mmHg) & $141.1 \pm 4.7$ & $130.5 \pm 3.6$ & 0.004 \\
DBP (mmHg) & $88.8 \pm 3.4$ & $84.5 \pm 3.0$ & 0.069 \\
Pulse pressure (mmHg) & $52.3 \pm 3.9$ & $46.0 \pm 2.0$ & 0.031 \\
FPG (mmol/l) & $5.7 \pm 0.2$ & $5.4 \pm 0.2$ & 0.146 \\
Heart rate at rest (beats/min) & $62.2 \pm 2.1$ & $59.6 \pm 2.2$ & 0.164 \\
Central SBP (mmHg) & $129.8 \pm 5.7$ & $121.0 \pm 4.7$ & 0.043 \\
Central DBP (mmHg) & $90.1 \pm 3.8$ & $85.8 \pm 3.6$ & 0.143 \\
Alx & $17.0 \pm 2.7$ & $19.1 \pm 3.2$ & 0.566 \\
SEVR (\%) & $1.69 \pm 0.08$ & $1.72 \pm 0.09$ & 0.579 \\
E/A & $0.82 \pm 0.06$ & $0.86 \pm 0.07$ & 0.358 \\
LVEF (\%) & $60.0 \pm 1.3$ & $61.9 \pm 1.0$ & 0.226 \\
LVESD (mm) & $33.0 \pm 0.8$ & $33.1 \pm 0.9$ & 0.913 \\
LVEDD (mm) & $48.5 \pm 0.6$ & $50.2 \pm 1.3$ & 0.099 \\
\hline Differences are considered statistically & & \\
& & &
\end{tabular}

Differences are considered statistically significant at $\mathrm{P}<0.05$. Data are mean \pm SEM. 


\section{COMBINED EXERCISE TRAINING EFFECTS}

Average compliance to the CT protocol was $95.1 \pm 1.1 \%$. CT significantly improved $\mathrm{VO}_{2 \max }$ with $13.1 \%$ (from $25.9 \pm 1.3$ to $29.3 \pm 1.5 \mathrm{ml} / \mathrm{min} / \mathrm{kg}, \mathrm{p}<0.001$ ), and $\mathrm{W}_{\max }$ with $20.0 \%$ (from $2.0 \pm 0.1$ to $2.4 \pm 0.1 \mathrm{~W} / \mathrm{kg}, \mathrm{p}<0.001$ ), whereas BMI did not significantly change (to $30.1 \pm 0.9 \mathrm{~kg} / \mathrm{m}^{2}, \mathrm{p}=0.159$ ).

The average MetS-score significantly dropped to $2.4 \pm 0.3$ at the end of the intervention period ( $p=0.003$ ) (figure 1 ). Nine of the 12 subjects decreased in number of components contributing to the MetS. Of these 9 subjects, 7 were cured from having the MetS. CT did not significantly change WC, TG, HDL-C, DBP and FPG (table 1). The intervention significantly reduced SBP with $7.5 \%$ (from $141.1 \pm 4.7$ to $130.5 \pm 3.6 \mathrm{mmHg}, p=0.004$ ). Also pulse pressure significantly decreased from 52.3 \pm 3.9 to $46.0 \pm 2.0 \mathrm{mmHg}(p=0.031$ ) (table 1$)$. Heart rate at rest was not significantly different between pre- and post-CT conditions (from $62.2 \pm 2.1$ to $59.6 \pm 2.2$ beats/min, $p=0.164$ ).

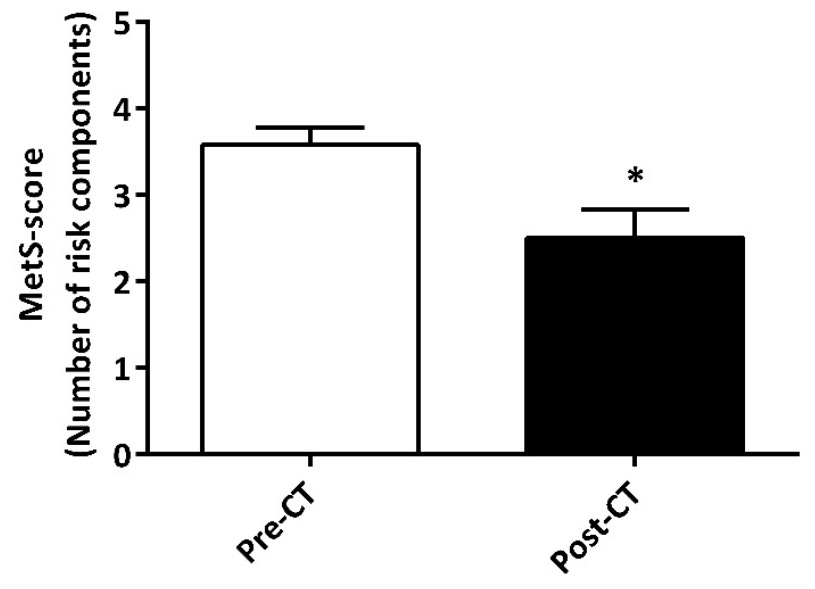

Figure 1. MetS-score pre(white column) and post(black column) CT. The value of the MetS-score was calculated as the sum of each participant his risk components of the MetS, $\mathrm{n}=12$. ${ }^{*} \mathrm{p}<0.05$ from pre-CT. Data are mean \pm SEM.

We measured aortic blood pressure and arterial stiffness with pulse wave analysis. Central SBP significantly decreased from $129.8 \pm 5.7$ to $121.0 \pm 4.7 \mathrm{mmHg}(p=0.043)$. Central DBP did not significantly change (from $90.1 \pm 3.8$ to $85.8 \pm 3.6 \mathrm{mmHg}$, $\mathrm{p}=0.143$ ) (table 1). Alx was not affected by $\mathrm{CT}$, with Alx being $17.0 \pm 2.7$ pre-training and $19.1 \pm 3.2$ post-training $(p=0.566)$ (table 1$)$. Also SEVR, as measure for 
myocardial perfusion relative to left ventricle workload, did not change with CT (from $1.69 \pm 0.08$ to $1.72 \pm 0.09 \%, p=0.579$ ). SEVR was above the critical $0.5 \%$ both pre- and post-CT, and thus subjects were not characterized by insufficient subendocardial perfusion.

Cardiac function was measured using echocardiography. CT did not improve diastolic function, as illustrated by unchanged E/A ratio (from $0.82 \pm 0.06$ to $0.86 \pm$ $0.07, \mathrm{p}=0.358$ ) and LVEF (from $60.0 \pm 1.3$ to $61.9 \pm 1.0 \%, \mathrm{p}=0.226$ ). Also systolic function was unaffected by CT, without changes in LVESD (from $33.0 \pm 0.8$ to 33.1 $\pm 0.9 \mathrm{~mm}, \mathrm{p}=0.913$ ) and LVEDD (from $48.5 \pm 0.6$ to $50.2 \pm 1.3 \mathrm{~mm}, \mathrm{p}=0.099$ ).

\section{DISCUSSION}

While BMI and FPG are often used as indicators of risk for the development of type 2 diabetes mellitus, MetS is associated with an increased risk for cardiovascular disease $^{2}$. Numerous studies have shown that combining diet with exercise training is very effective in the prevention of Met ${ }^{10}$. The limited amount of studies investigating the effect of exercise per se focused on aerobic exercise training. However, adding resistance exercise to aerobic exercise training is known to ameliorate the beneficial effects of exercise training on cardio-metabolic parameters ${ }^{16}$. In this study with overweight and obese men with MetS, we found that $\mathrm{CT}$ for 12 weeks improved the metabolic profile. CT formally reduced the number of people with MetS as hypertension, one of the components contributing to MetS, did no longer exceed the cut-off level. Although the cardio-metabolic profile improved after $\mathrm{CT}, \mathrm{CT}$ had no effect on indices for cardiac function and arterial stiffness.

Aerobic exercise training has been previously shown to be effective in reducing the metabolic risk in people with MetS. In a large group of 171 overweight, sedentary adults, 8 months of low-amount moderate-intensity and high-amount vigorousintensity exercise training decreased the MetS-score significantly ${ }^{29}$. Wang et al. ${ }^{30}$ found that 12 weeks of aerobic exercise training significantly decreased the MetSscore in postmenopausal women compared to a non-exercising group. Furthermore, they found that $17 \%$ of the participants converted to a non-MetS 
status after 12 weeks of aerobic exercise training. In a group of males and females $31 \%$ of the participants with MetS at baseline did no longer have MetS after 20 weeks of supervised aerobic exercise training ${ }^{31}$. Tjønna et al. ${ }^{12}$ reported $45 \%$ of MetS subjects to be cured after 16 weeks of aerobic interval training. One year of aerobic exercise training reduced the prevalence of MetS by $24 \%$ among middleaged men with Met $\mathrm{S}^{32}$. In line with these previous results upon aerobic exercise training, we found that CT without targeted weight loss reduced MetS-score by 31 $\%$. We further observed that $58 \%$ of the subjects converted to a non-MetS status after 12 weeks of CT. Only 2 other studies investigated the effect of CT on MetS. Steward et al..$^{33}$ investigated the effect of 6 months supervised CT in older men and women with mildly elevated blood pressure. Comparably to our results, they found that $40 \%$ of the participants who were diagnosed with MetS at baseline converted to a non-MetS status after CT. In postmenopausal women with MetS, 12 months of CT decreased MetS-score by $11 \%^{34}$. The milder effect in this study might be explained by the lower compliance to the CT protocol compared to our study; exercise attendance was $74 \%$ for the joint exercise sessions and $41 \%$ for the homebased exercise sessions, while in our study compliance to the CT protocol was 95 $\%$. Thus, our results show that $\mathrm{CT}$ might be as effective as aerobic exercise training in reducing the metabolic risk in people with MetS.

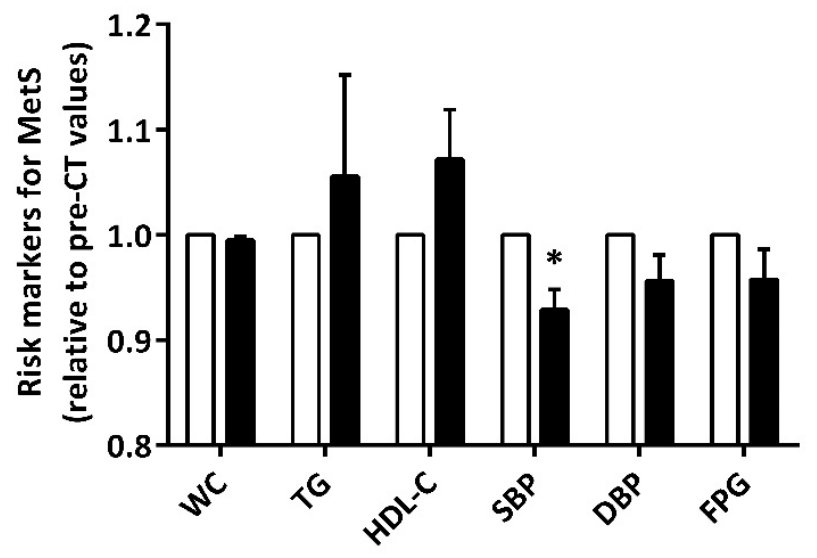

Figure 2. Changes in traditional risk components of the MetS upon $\mathrm{CT}$, relative to pre-CT values. Pre-CT white columns, post-CT black columns, $n=12 . \quad * p<0.05$ from pre-CT. Data are mean \pm SEM.

The positive effect of CT on MetS in our study was especially due to a CT-lowering effect on blood pressure. This is interesting, because in middle-aged and older subjects blood pressure is strongly and directly related to vascular and overall 
mortality ${ }^{35}$. We found that peripheral blood pressure was elevated in all 12 subjects before onset of the exercise training intervention, and systolic blood pressure decreased by $7.5 \%$ on average upon CT $(-10.6 \mathrm{mmHg})$. A recent meta-analysis ${ }^{36}$ investigated the effect of exercise training on peripheral blood pressure. Aerobic, resistance and combined aerobic and resistance exercise training studies were included, and exercise training intervention periods ranged from 4 to 52 weeks. This analysis showed that aerobic exercise training $(-3.5 \mathrm{mmHg})$ and resistance exercise training $(-1.8 \mathrm{mmHg}$ for dynamic resistance training, $-10.9 \mathrm{mmHg}$ for isometric resistance training) were very effective in reducing systolic blood pressure. The meta-analysis did not show a significant effect of CT on systolic blood pressure, however. This would suggest that CT may be less effective in lowering systolic blood pressure, and this would conflict with our observations. However, only 5 studies used CT to investigate effects on blood pressure, and 4 of these studies measured the effect of $\mathrm{CT}$ in subjects who did not suffer from hypertension. The other study, conducted by Laterza et $a .^{37}$, investigated the effect of CT in hypertensive and normotensive middle-aged subjects. They found that CT significantly reduced systolic blood pressure in hypertensive (from 145 to $130 \mathrm{mmHg}$ ), but not in normotensive subjects. Thus, the effect of CT on blood pressure might be limited to hypertensive people.

Arterial stiffness is regarded a predictor of increased cardiovascular risk and allcause mortality ${ }^{17-19}$. Alx is a non-invasive measure of arterial stiffness and is inversely associated with blood pressure ${ }^{38}$. Nevertheless, we did not find differences in Alx upon 12 weeks of CT. A recent meta-analysis ${ }^{39}$ investigated the effects of aerobic, resistance and combined exercise training on Alx. In the analysis, the median duration of structured exercise training was 12 weeks. It was found that Alx significantly improved after aerobic exercise training, but not after resistance exercise training. Only one study investigated the effect of CT on Alx previously ${ }^{40}$. In this study, 12 weeks of moderate-intensity CT did not change the Alx significantly in 97 overweight and obese, sedentary, middle-aged subjects. Our data adds that even in people with MetS CT does not affect Alx, and that blood pressure decreases in the absence of changes in arterial stiffness. This is in agreement with a previous finding upon resistance exercise training, where obese young men after 12 weeks of resistance training decreased systolic and diastolic blood pressure, without changes in Alx ${ }^{41}$. Thus, although there is a relationship between blood pressure and 
vascular function, CT seems to lower blood pressure independent of changes in arterial stiffness.

Diastolic or systolic cardiac function was unaffected upon CT training in the current study. This is in agreement with an earlier study in sedentary, elderly women who had a LVEF of $69.5 \%$ at baseline, and where 36 weeks of aerobic exercise training had no effect on ejection fraction, with an average LVEF post-exercise value of 69.6 $\%^{42}$. While it is generally accepted that physical activity training increases cardiac mass and ejection fraction on the long-term ${ }^{43}$, these changes may be too small to be measurable after 12 weeks of exercise training. We previously showed that 12 weeks of supervised CT improved LVEF very slightly, but significantly from $52.2 \pm$ 1.3 to $54.2 \pm 1.2 \%$ in 14 healthy, overweight/obese middle-aged men ${ }^{9}$. This small difference was measured by using magnetic resonance imaging (MRI). Cardiac MRI is the gold standard method to measure LVEF, and echocardiography is known to be a less reproducible and accurate measure of LVEF compared to cardiac MRI ${ }^{44}$. In the current study, echocardiography was used with slightly higher variation because this enabled us to monitor diastolic function (E/A ratio), which could mean that such small improvements may not have been detectable.

In summary, we conclude that CT improves the metabolic profile of overweight and obese men with MetS. CT formally reduced the number of people with MetS as hypertension, one of the components contributing to MetS, did no longer exceed the cut-off level. Although the positive effect on cardio-metabolic, CT does not improve cardiac function and arterial stiffness. 


\section{REFERENCES}

1. Ng, M., et al. Global, regional, and national prevalence of overweight and obesity in children and adults during 1980-2013: a systematic analysis for the Global Burden of Disease Study 2013. Lancet 384, 766-781 (2014).

2. Grundy, S.M., Brewer, H.B., Jr., Cleeman, J.I., Smith, S.C., Jr. \& Lenfant, C. Definition of metabolic syndrome: report of the National Heart, Lung, and Blood Institute/American Heart Association conference on scientific issues related to definition. Arterioscler Thromb Vasc Biol 24, e13-18 (2004).

3. Alberti, K.G., Zimmet, P. \& Shaw, J. Metabolic syndrome--a new world-wide definition. A Consensus Statement from the International Diabetes Federation. Diabet Med 23, 469-480 (2006).

4. Lennie, T.A. Cardiology patient page. The metabolic syndrome. Circulation 114, e528-529 (2006).

5. Hildrum, B., Mykletun, A., Hole, T., Midthjell, K. \& Dahl, A.A. Age-specific prevalence of the metabolic syndrome defined by the International Diabetes Federation and the National Cholesterol Education Program: the Norwegian HUNT 2 study. BMC Public Health 7, 220 (2007).

6. Esposito, K. \& Giugliano, D. Obesity, the metabolic syndrome, and sexual dysfunction in men. Clin Pharmacol Ther 90, 169-173 (2011).

7. Grundy, S.M., Hansen, B., Smith, S.C., Jr., Cleeman, J.I. \& Kahn, R.A. Clinical management of metabolic syndrome: report of the American Heart Association/National Heart, Lung, and Blood Institute/American Diabetes Association conference on scientific issues related to management. Circulation 109, 551-556 (2004).

8. Meex, R.C., et al. Restoration of muscle mitochondrial function and metabolic flexibility in type 2 diabetes by exercise training is paralleled by increased myocellular fat storage and improved insulin sensitivity. Diabetes 59, 572-579 (2010).

9. Schrauwen-Hinderling, V.B., et al. Improved ejection fraction after exercise training in obesity is accompanied by reduced cardiac lipid content. J Clin Endocrinol Metab 95, 1932-1938 (2010).

10. Roberts, C.K., Hevener, A.L. \& Barnard, R.J. Metabolic syndrome and insulin resistance: underlying causes and modification by exercise training. Compr Physiol 3, 1-58 (2013). 
11. Stensvold, D., et al. Strength training versus aerobic interval training to modify risk factors of metabolic syndrome. J Appl Physiol (1985) 108, 804810 (2010).

12. Tjonna, A.E., et al. Aerobic interval training versus continuous moderate exercise as a treatment for the metabolic syndrome: a pilot study. Circulation 118, 346-354 (2008).

13. Irving, B.A., et al. Effect of exercise training intensity on abdominal visceral fat and body composition. Med Sci Sports Exerc 40, 1863-1872 (2008).

14. Dumortier, M., et al. Low intensity endurance exercise targeted for lipid oxidation improves body composition and insulin sensitivity in patients with the metabolic syndrome. Diabetes Metab 29, 509-518 (2003).

15. Pal, S., Radavelli-Bagatini, S. \& Ho, S. Potential benefits of exercise on blood pressure and vascular function. J Am Soc Hypertens 7, 494-506 (2013).

16. Marzolini, S., Oh, P.I. \& Brooks, D. Effect of combined aerobic and resistance training versus aerobic training alone in individuals with coronary artery disease: a meta-analysis. Eur J Prev Cardiol 19, 81-94 (2012).

17. Vlachopoulos, C., Aznaouridis, K. \& Stefanadis, C. Clinical appraisal of arterial stiffness: the Argonauts in front of the Golden Fleece. Heart 92, 1544-1550 (2006).

18. Laurent, S., et al. Expert consensus document on arterial stiffness: methodological issues and clinical applications. Eur Heart J 27, 2588-2605 (2006).

19. Agabiti-Rosei, E., et al. Central blood pressure measurements and antihypertensive therapy: a consensus document. Hypertension 50, 154160 (2007).

20. O'Rourke, M.F. \& Gallagher, D.E. Pulse wave analysis. J Hypertens Supp/ 14, S147-157 (1996).

21. Vlachopoulos, C., et al. Prediction of cardiovascular events and all-cause mortality with central haemodynamics: a systematic review and metaanalysis. Eur Heart J 31, 1865-1871 (2010).

22. Brouwers, B., Schrauwen-Hinderling, V.B., Gemmink, A., Havekes, B., Bruls, Y., Dahlmans, D., Hesselink, M.K.C., Schrauwen, P. Exercise training reduces liver fat content and improves peripheral, but not hepatic insulin sensitivity 
in overweight and obese, middle-aged men with non-alcoholic fatty liver disease. in prep.

23. Kuipers, H., Verstappen, F.T., Keizer, H.A., Geurten, P. \& van Kranenburg, $G$. Variability of aerobic performance in the laboratory and its physiologic correlates. Int J Sports Med 6, 197-201 (1985).

24. Solymoss, B.C., et al. Effect of increasing metabolic syndrome score on atherosclerotic risk profile and coronary artery disease angiographic severity. Am J Cardiol 93, 159-164 (2004).

25. Vidal, J., et al. The importance of diagnostic criteria in the association between the metabolic syndrome and cardiovascular disease in obese subjects. Int J Obes (Lond) 29, 668-674 (2005).

26. O'Rourke, M.F. \& Pauca, A.L. Augmentation of the aortic and central arterial pressure waveform. Blood Press Monit 9, 179-185 (2004).

27. Salvi, P., et al. Changes in subendocardial viability ratio with acute highaltitude exposure and protective role of acetazolamide. Hypertension 61, 793-799 (2013).

28. Friedewald, W.T., Levy, R.I. \& Fredrickson, D.S. Estimation of the concentration of low-density lipoprotein cholesterol in plasma, without use of the preparative ultracentrifuge. Clin Chem 18, 499-502 (1972).

29. Johnson, J.L., et al. Exercise training amount and intensity effects on metabolic syndrome (from Studies of a Targeted Risk Reduction Intervention through Defined Exercise). Am J Cardiol 100, 1759-1766 (2007).

30. Wang, C.H., Chung, M.H., Chan, P., Tsai, J.C. \& Chen, F.C. Effects of endurance exercise training on risk components for metabolic syndrome, interleukin-6, and the exercise capacity of postmenopausal women. Geriatr Nurs 35, 212-218 (2014).

31. Katzmarzyk, P.T., et al. Targeting the metabolic syndrome with exercise: evidence from the HERITAGE Family Study. Med Sci Sports Exerc 35, 17031709 (2003).

32. Anderssen, S.A., Carroll, S., Urdal, P. \& Holme, I. Combined diet and exercise intervention reverses the metabolic syndrome in middle-aged males: results from the Oslo Diet and Exercise Study. Scand J Med Sci Sports 17, 687-695 (2007). 
33. Stewart, K.J., et al. Exercise and risk factors associated with metabolic syndrome in older adults. Am J Prev Med 28, 9-18 (2005).

34. Kemmler, W., Von Stengel, S., Engelke, K. \& Kalender, W.A. Exercise decreases the risk of metabolic syndrome in elderly females. Med Sci Sports Exerc 41, 297-305 (2009).

35. Lewington, S., Clarke, R., Qizilbash, N., Peto, R. \& Collins, R. Age-specific relevance of usual blood pressure to vascular mortality: a meta-analysis of individual data for one million adults in 61 prospective studies. Lancet 360, 1903-1913 (2002).

36. Cornelissen, V.A. \& Smart, N.A. Exercise training for blood pressure: a systematic review and meta-analysis. J Am Heart Assoc 2, e004473 (2013).

37. Laterza, M.C., et al. Exercise training restores baroreflex sensitivity in never-treated hypertensive patients. Hypertension 49, 1298-1306 (2007).

38. DeLoach, S.S. \& Townsend, R.R. Vascular stiffness: its measurement and significance for epidemiologic and outcome studies. Clin J Am Soc Nephrol 3, 184-192 (2008).

39. Ashor, A.W., Lara, J., Siervo, M., Celis-Morales, C. \& Mathers, J.C. Effects of exercise modalities on arterial stiffness and wave reflection: a systematic review and meta-analysis of randomized controlled trials. PLoS One 9, e110034 (2014).

40. Ho, S.S., Radavelli-Bagatini, S., Dhaliwal, S.S., Hills, A.P. \& Pal, S. Resistance, aerobic, and combination training on vascular function in overweight and obese adults. J Clin Hypertens (Greenwich) 14, 848-854 (2012).

41. Croymans, D.M., et al. Effects of resistance training on central blood pressure in obese young men. J Hum Hypertens 28, 157-164 (2014).

42. Park, S.K., Park, J.H., Kwon, Y.C., Yoon, M.S. \& Kim, C.S. The effect of longterm aerobic exercise on maximal oxygen consumption, left ventricular function and serum lipids in elderly women. J Physiol Anthropol Appl Human Sci 22, 11-17 (2003).

43. Gielen, S., Laughlin, M.H., O'Conner, C. \& Duncker, D.J. Exercise training in patients with heart disease: review of beneficial effects and clinical recommendations. Prog Cardiovasc Dis 57, 347-355 (2015).

44. Dorosz, J.L., Lezotte, D.C., Weitzenkamp, D.A., Allen, L.A. \& Salcedo, E.E. Performance of 3-dimensional echocardiography in measuring left 
ventricular volumes and ejection fraction: a systematic review and metaanalysis. J Am Coll Cardiol 59, 1799-1808 (2012). 




\section{CHAPTER 8}

\section{THE DUAL FACES OF EXERCISE TRAINING ON WHOLE BODY INSULIN SENSITIVITY}

Bram Brouwers, Ruth C.M. Meex, Vera B. Schrauwen-Hinderling, Esther Phielix, Bas Havekes, Patrick Schrauwen, Matthijs K.C. Hesselink

In preparation for submission 


\section{ABSTRACT}

Whereas physical exercise training is one of the most advocated routes to promote whole body insulin sensitivity, not all participants benefit similarly. Here we aimed to identify metabolic characteristics or responses that distinguished subjects who failed to promote whole body insulin sensitivity upon exercise training (nonresponders, NON-RESP) from subjects who did improve insulin sensitivity (responders, RESP).

Forty-one overweight/obese subjects and 30 type 2 diabetic (T2DM) patients, all men, were included in this analysis. All subjects participated in a 12 week supervised progressive, combined aerobic and resistance exercise training protocol. Pre- and post-training cardiorespiratory fitness $\left(\mathrm{VO}_{2 \max }, \mathrm{W}_{\max }\right)$ and muscle strength (1RM) were measured. Whole body insulin sensitivity was measured (hyperinsulinemic-euglycemic clamp) before and after training and used for stratification of RESP and NON-RESP. An array of parameters related to physical fitness and metabolic health were measured. Retrospectively, 17 subjects (23.9\%) were classified as NON-RESP whereas 54 subjects as RESP based on exercise training induced changes in glucose infusion rate $(\triangle G I R)$.

$\operatorname{RESP}(G I R+23.7 \pm 2.2 \%, p<0.001)$ and NON-RESP $(G I R-20.7 \pm 3.5 \%, p<0.001)$ did not differ in any of the baseline parameters measured (age $58.6 \pm 0.8$ and $59.2 \pm$ 1.1 year in RESP and NON-RESP, $p=0.739$ ). Exercise training increased $\mathrm{VO}_{2 \max }$ $(p<0.001), W_{\max }(p<0.001)$ and $1 R M(p<0.001)$ similarly in RESP and NON-RESP. Also muscle mitochondrial content in NON-RESP increased similarly compared to RESP upon exercise training. Body composition related parameters were unaffected by the exercise training program, except for a modest but significant drop in fat mass over time $(p=0.006)$. In the RESP group, training mediated improvements in whole body insulin sensitivity did translate in a significant drop in fasting plasma glucose (FPG) (from $7.0 \pm 0.3$ to $6.6 \pm 0.3 \mathrm{mmol} / \mathrm{l}, \mathrm{p}<0.001$ ) and an almost significant decrease in plasma triglycerides (TG) levels (from $1.59 \pm 0.09$ to $1.46 \pm 0.09 \mathrm{mmol} / \mathrm{l}$, $\mathrm{p}=0.051$ ) after training, whereas in the NON-RESP group FPG increased (from $7.4 \pm$ 0.5 to $8.0 \pm 0.7 \mathrm{mmol} / \mathrm{l}, \mathrm{p}<0.001$ ) along with a striking increase in plasma TG posttraining (from $1.46 \pm 0.08$ to $1.90 \pm 0.16 \mathrm{mmol} / \mathrm{l}, \mathrm{p}<0.001$ ).

In conclusion, in this group of overweight/obese subjects and T2DM patients, 23.9 $\%$ of subjects failed to respond to a 12 week exercise training program with respect to whole body insulin sensitivity. Classical parameters of physical fitness and muscle 
strength, including skeletal muscle mitochondrial mass, increased similarly in RESP and NON-RESP. We could not identify any differences in baseline parameters, which could be at the base of the differential response to exercise training. Most strikingly, FPG and plasma TG levels decreased in the RESP group, whereas both increased in the NON-RESP group after exercise training. The mechanism underlying this differential response remains to be elucidated. Therefore, more research is warranted to understand why some people do, and other do not, improve insulin sensitivity upon exercise training. 



\section{INTRODUCTION}

Exercise training is a cornerstone in prevention and treatment of insulin resistance and type 2 diabetes mellitus (T2DM) and likewise is advocated as first line treatment by the American Diabetes Association ${ }^{1}$. Exercise training has been shown to improve insulin sensitivity and glycemic control ${ }^{2}$ and medical practitioners routinely prescribe exercise to patients with - and to people at risk for - T2DM, as a means to improve their metabolic health ${ }^{3}$.

Despite the known favorable effects of exercise training at the group level, not all individuals do indeed benefit from exercise training with respect to metabolic parameters ${ }^{4,5}$. As a matter of fact, approximately $15-20 \%$ of individuals fail to improve glucose homeostasis, insulin sensitivity, and/or muscle mitochondrial density after supervised exercise training interventions ${ }^{5,6}$. Differences in exercise training responses could be attributed to a too low exercise intensity, too low volume of exercise or the wrong type of exercise (resistance exercise versus aerobic exercise) $)^{7}$. In respect to the latter, combining aerobic and resistance exercise training has been suggested to increase the beneficial effects of exercise training on cardio-metabolic parameters ${ }^{8}$. Results from a large-scale randomized control trial revealed that (with iso-energetic exercise programs) $\mathrm{HbA}_{1 \mathrm{c}}$ levels improved only in patients undergoing a combined aerobic-resistance type of exercise training program ${ }^{9}$. Nevertheless, even upon combined exercise training, Stephens et al. ${ }^{5,10}$ reported a $21 \%$ non-responsiveness with respect to glucose homeostasis and muscle substrate metabolism after 9 months of supervised aerobic, resistance, or combined exercise training.

Thus, other determinants than the exercise program itself seem to play a role in the responsiveness to exercise training. It has been reported that the level of T2DM prevention upon a lifestyle intervention (compromising exercise training) closely associates with the amount of weight lost during the intervention ${ }^{11,12}$. However, some studies also observed significant improvements in whole body insulin sensitivity upon exercise training in the absence of relevant changes in body mass ${ }^{13}$. Moreover, it is unclear if relevant differences exist in baseline characteristics of people who do not improve insulin sensitivity upon exercise training versus subjects who do improve. Furthermore, it is not known if these baseline differences 
somehow help to explain the non-responsiveness. Neither has it been systematically examined whether or not other metabolic parameters do respond to exercise training in subjects who fail to improve insulin sensitivity upon exercise training. Therefore, we examined baseline characteristics and evaluated the impact of a supervised, progressive combined aerobic and resistance type of exercise training program on cardiorespiratory fitness, body composition, tissue-specific insulin sensitivity, metabolic flexibility, mitochondrial density and plasma markers of glucose and lipid metabolism in overweight/obese subjects and T2DM patients, who were categorized as responders or non-responders based upon improvements in whole body insulin sensitivity upon exercise training.

\section{RESEARCH DESIGN AND METHODS}

\section{SUBJECT RECRUITMENT AND STRATIFICATION}

Thirty-seven subjects who participated in the study of Meex et al. ${ }^{13}$ and 34 subjects who participated in the study of Brouwers et al. ${ }^{14}$ were included in this analysis, resulting in a total number of 71 subjects. Subjects were sedentary, middle-aged (40-70 years old) with stable dietary habits. In total, 30 subjects (17 subjects from Meex et al. and 13 subjects from Brouwers et al.) had been diagnosed with T2DM. T2DM patients were not on insulin treatment, but were allowed to use oral glucose lowering medication for at least 6 months - with constant dose for at least 2 months - or on a dietary treatment for at least 6 months, with fasting plasma glucose concentrations $\geq 7.0 \mathrm{mmol} / \mathrm{l}$ and $<10.0 \mathrm{mmol} / \mathrm{l}$ at the time of screening. Subjects were recruited via advertisements in local newspapers. All subjects gave written informed consent before participation in the study. The Maastricht University Medical Ethical Committee approved the study. The study was performed according to the principles expressed in the most recent version of the declaration of Helsinki.

Physical fitness was examined in a graded cycling test on a stationary bike until exhaustion during which maximal work load $\left(\mathrm{W}_{\max }\right)$ and maximal aerobic capacity $\left(\mathrm{VO}_{2 \max }\right)$ were assessed ${ }^{15}$. Maximal strength for the larger muscle groups was measured as one-repetition maximum (1RM), for details see Meex et $a l^{13}$. Body 
composition was determined by hydrostatic weighing ${ }^{16}$ or DXA (Hologic Discovery A, Waltham, MA, USA).

Retrospectively, the entire group of 71 subjects was stratified according to improvement (responders, RESP) or no improvement (non-responders, NON-RESP) in whole body insulin sensitivity upon exercise training. The increase in glucose infusion rate (GIR) required to maintain euglycemia upon infusion of insulin (40 $\mathrm{mU} / \mathrm{m}^{2} / \mathrm{min}$ ) was measured to assess whole body insulin sensitivity. The difference in $\mathrm{GIR}$ before and after exercise training $(\Delta G I R)$ was used to stratify for the response of whole body insulin sensitivity to the exercise training program. Stratification resulted in a group of 54 subjects who possessed a positive $\triangle$ GIR (RESP) and 17 subjects who possessed a negative $\triangle G I R$ after exercise training (NON-RESP).

\section{EXERCISE TRAINING PROTOCOL}

Subjects were engaged in a supervised progressive, combined aerobic and resistance exercise training program as previously described ${ }^{13,14}$. In short, subjects performed a tightly controlled exercise training program for 12 weeks. Aerobic exercise was carried out on a cycling ergometer twice a week for $30 \mathrm{~min}$ and resistance exercise was performed once a week and focused on large muscle. Every 4-6 weeks, $1 \mathrm{RM}$ and $\mathrm{VO}_{2 \max }$ were reassessed, and training loads were readjusted accordingly. Supervised training sessions were performed with 3-4 subjects at a time.

\section{HYPERINSULINEMIC-EUGLYCEMIC CLAMP}

A primed-continuous infusion of $\left[6,6-{ }^{2} \mathrm{H}_{2}\right]$ glucose was used to determine noninsulin-stimulated rates of endogenous glucose production (EGP), glucose appearance $\left(R_{a}\right)$ and whole body glucose disposal rates $\left(R_{d}\right)$ as previously described ${ }^{17}$. Insulin sensitivity was measured by a $3 \mathrm{~h}$ hyperinsulinemic-euglycemic clamp ( $\left.40 \mathrm{mU} / \mathrm{m}^{2} / \mathrm{min}\right)^{13}$ or a 2-step $6 \mathrm{~h}$ hyperinsulinemic-euglycemic clamp (10 and $\left.40 \mathrm{mU} / \mathrm{m}^{2} / \mathrm{min}\right)^{14}$, before and after the exercise training period, as previously described. 
Isotopic enrichment of plasma glucose was determined by electric impact ionization gas chromatography-mass spectroscopy as previously described ${ }^{18}$. Steele's single-pool steady state equations were used to calculate $R_{a}$ and $R_{d}{ }^{19}$. Volume of distribution was assumed to be $0.160 \mathrm{l} / \mathrm{kg}$ for glucose. Insulin-stimulated glucose disposal (delta $R_{d}$ ) was computed as the difference between $R_{d}$ under insulin-stimulated conditions and $R_{d}$ under basal and non-insulin-stimulated conditions. EGP was calculated as $R_{a}$ - exogenous glucose infusion rate.

\section{BLOOD SAMPLE ANALYSIS}

On the hyperinsulinemic-euglycemic clamp test day, overnight fasted arterialized blood samples were collected and immediately centrifuged at high speed. Plasma was frozen in liquid nitrogen and stored at $-80{ }^{\circ} \mathrm{C}$ until assayed. Plasma free fatty acids and fasting plasma glucose were measured with enzymatic assays automated on a Cobas Fara/Mira (FFA: Wako Nefa C test kit; Wako Chemicals, Neuss, Germany) (FPG: hexokinase method; La Roche, Basel, Switzerland) or spectrophotometrically ${ }^{13}$. Plasma triglycerides (TG) were measured colorimetrically (Roche, Vienna, Austria) or enzymatically (Sigma-Aldrich Chemie, Steinheim, Germany). Total cholesterol (Roche Diagnostics, Mannheim, Germany) and high density lipoprotein cholesterol (HDL-C) (Roche Diagnostics, Mannheim, Germany), after precipitation of apolipoprotein B containing lipoproteins with phosphotungstic acid and magnesium ions, were analyzed in serum enzymatically (GPO-Trinder; Sigma-Aldrich Corp., St. Louis, MO, USA). Low density lipoprotein cholesterol (LDL-C) was calculated according to the Friedewald equation ${ }^{20}$, with no subjects having plasma TG levels above $4.5 \mathrm{mmol} / \mathrm{l}$. 


\section{MUSCLE BIOPSY AND ANALYSIS}

Muscle biopsies were taken from the musculus vastus lateralis, prior to the clamp under local anesthesia ( $2 \%$ xylocaine), according to the Bergstrom technique ${ }^{21}$. Any visible non-muscle material was dissected from muscle tissue. Samples were stored at $-80^{\circ} \mathrm{C}$ until further use.

Mitochondrial density was assessed, as previously described ${ }^{13}$. In brief, a monoclonal antibody cocktail detecting structural proteins in any of the 5 complexes of the oxidative phosphorylation system (MS601, Mitosciences, Eugene, OR, USA) was used for western blotting of muscle lysates. Gels were loaded with equal amounts of protein of pre- and post-exercise training lysates to allow valid comparison between pre- and post-exercise training samples. To adjust for intergel variation, the optical density of the band of interest per subject was normalized to the mean optical density of the complete gel. Protein content is expressed as arbitrary units $(\mathrm{AU})$.

\section{STATISTICS}

All values are reported as the mean \pm standard error of the mean (SEM). Statistical analysis was performed two-sided using IBM SPSS 21 (SPSS, Chicago, IL, USA). Statistical significance was set at $P<0.05$. Subject baseline characteristics were analyzed using one-way ANOVA with Tukey or Games-Howell post-hoc correction. A Mann-Whitney $U$ test was run to determine if there was a difference in group composition (overweight/obese subjects versus T2DM patients) between RESP and NON-RESP. Exercise training effects were analyzed using a two-way ANOVA model for repeated measures using RESP and NON-RESP as between-subject variables and pre- and post-training data as repeated within-subject variables. A Pearson's correlation was run to assess the relationship between $\Delta \mathrm{GIR}$ and changes upon exercise training $(\Delta)$ in other outcome parameters. 


\section{RESULTS}

\section{SUBJECTS}

Seventy-one subjects were included in this analysis. Of these 71 subjects, 54 improved their GIR (average $\Delta \mathrm{GIR}$ of $+23.7 \pm 2.2 \%$ ) upon exercise training (RESP) whereas 17 subjects ( $23.9 \%$ ) decreased their GIR (average $\Delta$ GIR of $-20.7 \pm 3.5 \%$ ) after exercise training (NON-RESP) (figure 1a). In the RESP group, 21 of the 54 subjects (38.9\%) had T2DM, which was not significantly different from the NONRESP group in which 9 of the 17 subjects ( $52.9 \%$ ) had been diagnosed with T2DM $(p=0.310)$. T2DM patients are represented by the green columns in figure 1 and 3 . RESP and NON-RESP did not differ in age, with an average age of $58.6 \pm 0.8$ year in RESP and $59.2 \pm 1.1$ year in NON-RESP $(p=0.740)$ (table 1). Compliance to the exercise training program was high and comparable between RESP and NON-RESP. Average attendance to the supervised exercise training sessions was $98.1 \pm 0.5 \%$ in RESP and $96.8 \pm 1.2 \%$ in NON-RESP $(p=0.245)$.

\section{CHANGES IN EXERCISE CAPACITY, MITOCHONDRIAL DENSITY AND BODY COMPOSITION}

Upon exercise training, $\mathrm{VO}_{2 \max }, \mathrm{W}_{\max }$ and $1 \mathrm{RM}$ significantly increased similarly over time (all $p<0.001)$ in both groups $\left(\Delta \mathrm{VO}_{2 \max } 10.1 \pm 1.2\right.$ and $7.8 \pm 3.1 \%, \Delta \mathrm{W}_{\max } 17.2 \pm$ 1.5 and $14.9 \pm 2.5 \%, \triangle 1 R M 21.4 \pm 1.4$ and $19.1 \pm 2.6 \%$ in RESP and NON-RESP, respectively, all $p>0.05$ for time*group) (table 1 ). Notwithstanding the significant increase in $\mathrm{VO}_{2 \max }$ upon exercise training in both groups, a few subjects in fact decreased $\mathrm{VO}_{2 \max }$ upon exercise training. These subjects, however, were not overrepresented in the NON-RESP group (figure $1 \mathrm{~b}$ ). Likewise, muscle strength robustly increased in all but 2 subjects (figure 1c). In line with these observations, exercise training mediated change in whole body glucose uptake did not correlate with changes in $\mathrm{VO}_{2 \max }$ or $1 \mathrm{RM}\left(\Delta \mathrm{GIR}\right.$ with $\Delta \mathrm{VO}_{2 \max }, \mathrm{r}=0.195, \mathrm{p}=0.117$ and $\Delta \mathrm{GIR}$ with $\Delta 1 \mathrm{RM}, \mathrm{r}=0.012, \mathrm{p}=0.923)$. 
Mitochondrial density was evaluated by measuring protein content of the 5 structural subunits of the 5 distinct complexes of the electron transport chain. In 13 out of 71 subjects, there was insufficient muscle sample left to perform the mitochondrial density assays. Of these 13 subjects, 3 were representing the NONRESP group and 10 the RESP group (figure 1d). Exercise training induced a time effect for all individual complexes and the mean protein content of these complexes. Thus, exercise training increased mitochondrial density comparably in both groups (table 2). No significant group or time*group effects was observed. In the complete group of subjects, exercise training induced changes in $\triangle$ GIR did not correlate with OXPHOS protein content ( $\triangle$ OXPHOS protein content) $(r=0.049$, $\mathrm{p}=0.714)$.

Although training did not alter body weight, BMI and fat free mass (table 1), we observed a significant change in body fat percentage and fat mass upon exercise training (time effect $p=0.004$ for body fat percentage and $p=0.006$ for body fat mass), which was not significantly different between RESP and NON-RESP ( $p=0.228)$ (figure 1e). In the complete group of subjects, exercise training induced changes in $\Delta G I R$ negatively correlated with fat mass ( $\Delta$ fat mass) $(r=-0.237, p=0.048)$ (figure $2 c$ ).

\section{BASELINE CHARACTERISTICS}

We next investigated whether differences in baseline parameters could explain the difference in $\triangle \mathrm{GIR}$ response upon exercise training. Before training, $\mathrm{VO}_{2 \max }, \mathrm{W}_{\max }$ and $1 \mathrm{RM}$ was comparable in RESP and NON-RESP $\left(\mathrm{VO}_{2 \max } 27.6 \pm 0.7\right.$ and $27.0 \pm 1.0$ $\mathrm{ml} / \mathrm{min} / \mathrm{kg}, \mathrm{W}_{\max } 2.1 \pm 0.1$ and $2.0 \pm 0.1 \mathrm{~W} / \mathrm{kg}, 1 \mathrm{RM} 84.0 \pm 2.1$ and $81.1 \pm 2.9 \mathrm{~kg}$ for RESP and NON-RESP, respectively, $p>0.05$ ) (table 1). For the 5 structural proteins in the 5 distinct complexes of the electron transport chain, we observed no differences between RESP and NON-RESP (table 2). Averaging total protein content of these different complexes also revealed no baseline differences between RESP and NON-RESP $(0.84 \pm 0.06$ and $0.72 \pm 0.08 \mathrm{AU}, \mathrm{p}=0.303$, respectively, table 2$)$. Also body weight, $\mathrm{BMI}$, fat mass, fat free mass and fat percentage was not different between RESP and NON-RESP (table 1). 


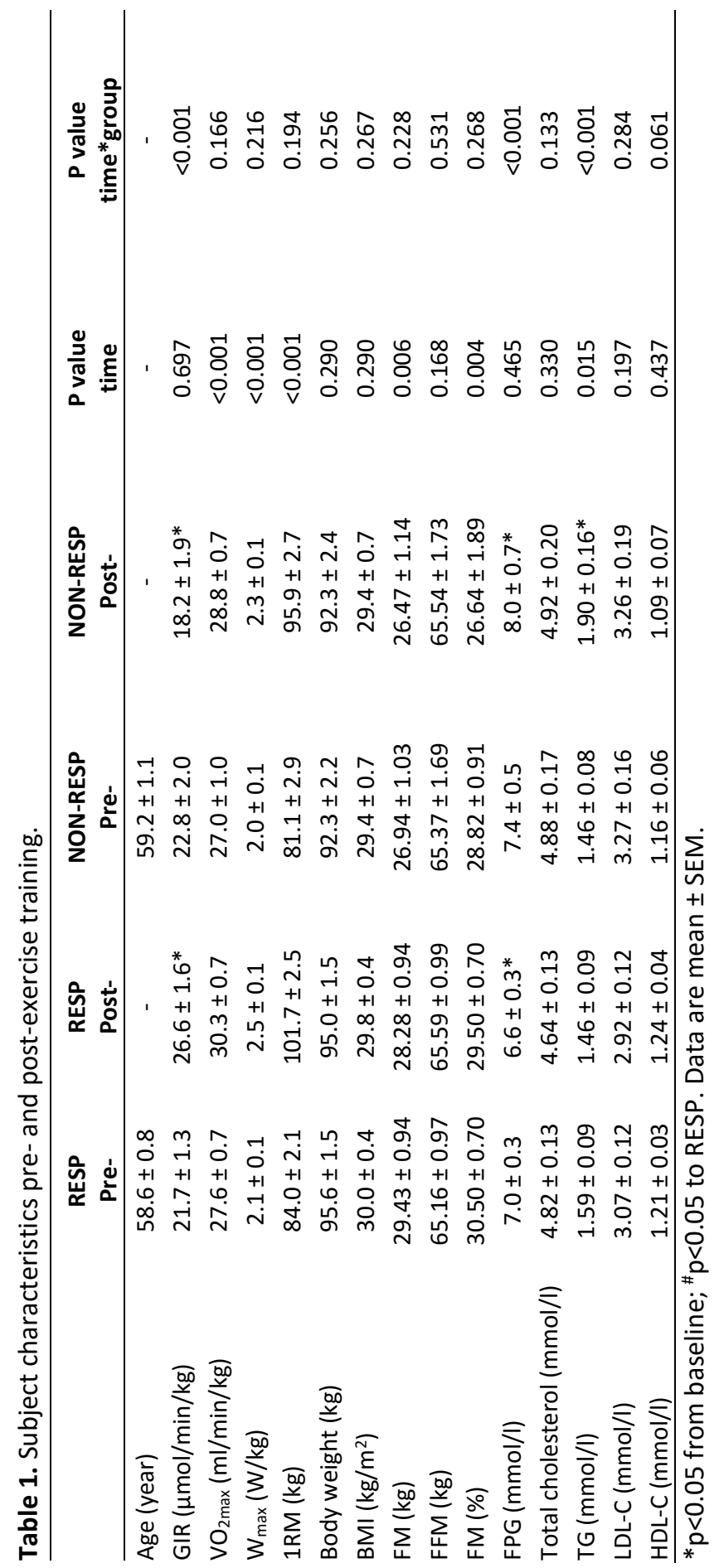


FPG levels were similar at the onset of the exercise training program $(7.0 \pm 0.3$ and $7.4 \pm 0.5 \mathrm{mmol} / \mathrm{l}, \mathrm{p}=0.417$, for RESP and NON-RESP, respectively). Total cholesterol $(p=0.718)$, plasma TG $(p=0.463), L D L-C(p=0.337)$ and HDL-C $(p=0.498)$ levels were comparable between groups at the start of the exercise training program (table 1). Also fasting FFA levels were comparable between RESP and NON-RESP (581.5 \pm 22.7, $586.8 \pm 34.1 \mu \mathrm{mol} / \mathrm{l}$, in RESP and NON-RESP, respectively, $p=0.905)$. Insulinmediated suppression of lipolysis, measured as the relative drop in plasma FFA upon $40 \mathrm{mU} / \mathrm{m}^{2} / \mathrm{min}$ insulin infusion, was $81.1 \pm 1.0 \%$ in RESP and $80.2 \pm 1.9 \%$ in NON-RESP, and this was not significantly different $(p=0.665)$.

GIR was comparable between groups pre-training $(21.7 \pm 1.3 \mu \mathrm{mol} / \mathrm{min} / \mathrm{kg}$ in RESP and $22.8 \pm 2.0 \mu \mathrm{mol} / \mathrm{min} / \mathrm{kg}$ in NON-RESP, $p=0.678$ ). Delta $R_{d}$, determined by the change in glucose disposal rate during the hyperinsulinemic-euglycemic clamp, was not significantly different between RESP and NON-RESP, with delta $R_{d}$ being $12.3 \pm$ $1.4 \mu \mathrm{mol} / \mathrm{min} / \mathrm{kg}$ in RESP and $12.2 \pm 2.4 \mu \mathrm{mol} / \mathrm{min} / \mathrm{kg}$ in NON-RESP $(p=0.960)$. Also insulin-stimulated suppression of EGP, determined as the relative suppression of EGP during the hyperinsulinemic-euglycemic clamp (delta EGP \%) upon 40 $\mathrm{mU} / \mathrm{m}^{2} / \mathrm{min}$ insulin infusion, was not significantly different between groups (-82.1 \pm 6.5 and $-91.1 \pm 7.0 \%, p=0.466$, in RESP and NON-RESP, respectively). Thus, neither peripheral nor central insulin sensitivity was different between the RESP and the NON-RESP group at the onset of the training program.

\section{CHANGES IN GLUCOSE AND LIPID PROFILE}

Twelve weeks of exercise training induced a time*group $(p<0.001)$, but no time $(p=0.465)$ or group $(p=0.100)$ effect on FPG levels (table 1$)$. While RESP possessed the anticipated drop in FPG levels (from $7.0 \pm 0.3$ to $6.6 \pm 0.3 \mathrm{mmol} / \mathrm{l}, p<0.001$ ), the reverse response was observed in the NON-RESP group; FPG increased significantly upon exercise training from $7.4 \pm 0.5$ to $8.0 \pm 0.7 \mathrm{mmol} / \mathrm{l}, \mathrm{p}=0.043$. The individual responses on FPG are depicted in figure $1 \mathrm{f}$. In the complete group of subjects, exercise training induced changes in $\triangle$ GIR negatively correlated with FPG ( $\triangle \mathrm{FGP}$ ) $(r=-0.415, p<0.001$, figure $2 a)$. 


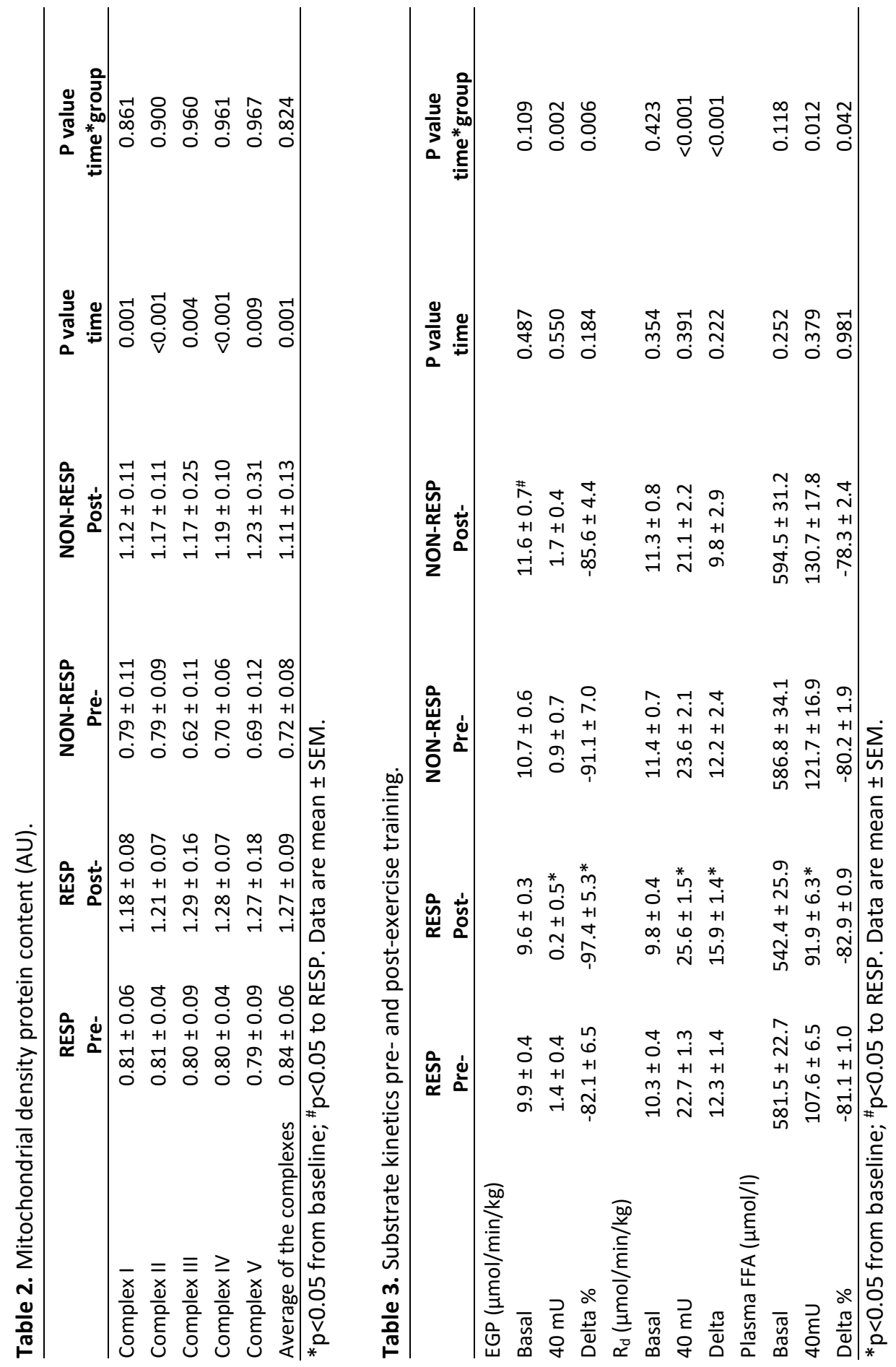



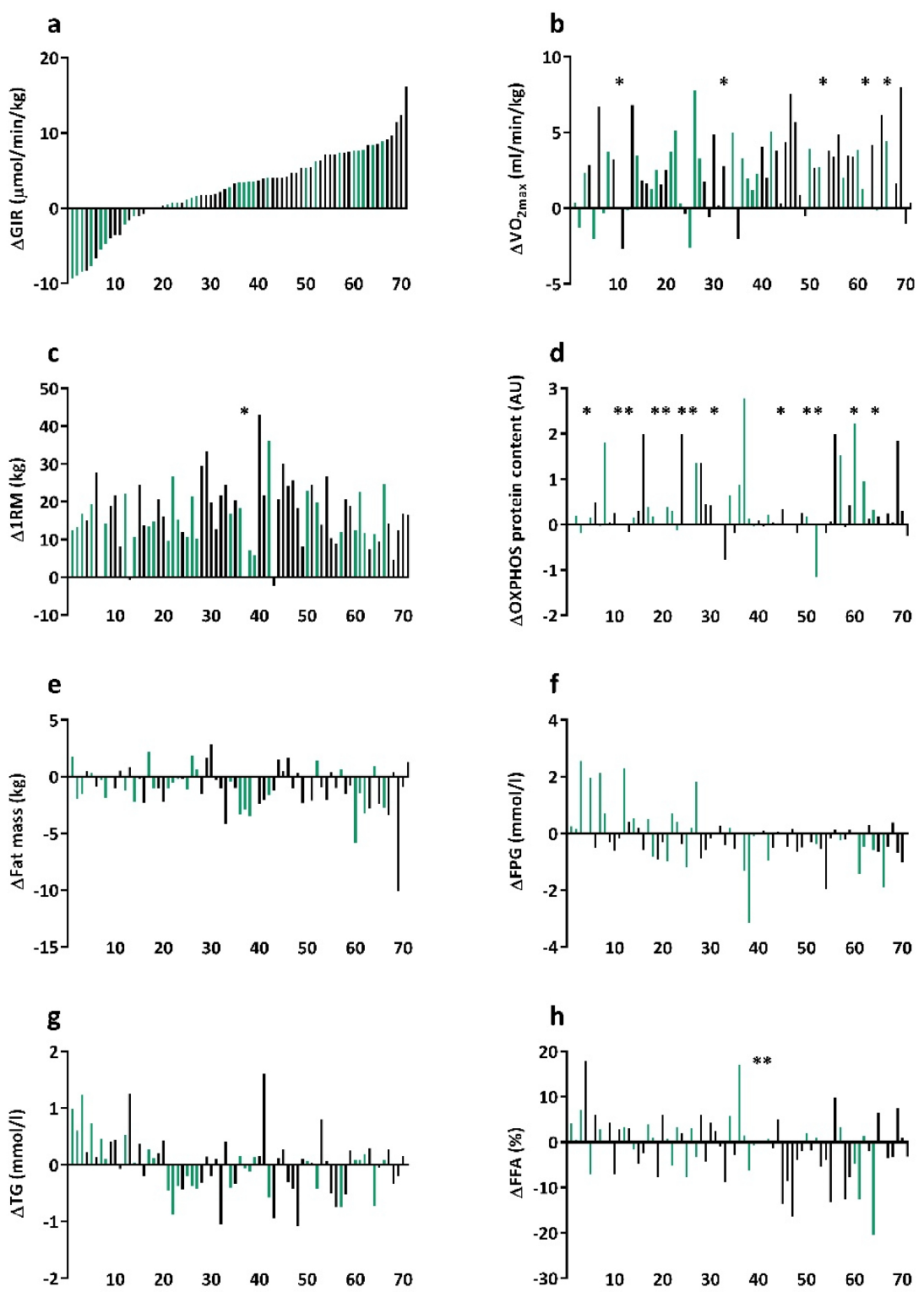

Figure 1. Individual data on changes in (a) glucose infusion rate $(\Delta G I R)$ during 40 $\mathrm{mU} / \mathrm{m}^{2} / \mathrm{min}$ and corresponding changes in (b) cardiorespiratory fitness $\left(\Delta \mathrm{VO}_{2 \mathrm{max}}\right)$, (c) one repeated maximum ( $\triangle 1 \mathrm{RM})$, (d) mitochondrial density ( $\triangle \mathrm{OXPHOS}$ protein content), (e) fat mass ( $\Delta$ fat mass), (f) fasting plasma glucose $(\Delta \mathrm{FPG}),(\mathrm{g})$ fasting plasma triglycerides $(\Delta T \mathrm{TG})$ and (h) fasting free fatty acid (FFA) levels. Green columns represent T2DM patients, black columns non-T2DM subjects. * represents missing data. 
Exercise training induced a time $(p=0.015)$ and time*group $(p<0.001)$ effect for plasma TG (table 1). Strikingly, plasma TG levels increased significantly upon exercise training in the NON-RESP group (from $1.46 \pm 0.08$ to $1.90 \pm 0.16 \mathrm{mmol} / \mathrm{l}$, $\mathrm{p}=0.001$ ), whereas plasma TG tended to decrease in the RESP group (from $1.59 \pm$ 0.09 to $1.46 \pm 0.09 \mathrm{mmol} / \mathrm{l}, \mathrm{p}=0.051$ ). The individual responses on plasma TG are depicted in figure $1 \mathrm{~g}$. In the complete group of subjects, exercise training induced changes $\Delta$ GIR negatively correlated with plasma TG $(\Delta T G)(r=-0.371, p=0.001$, figure $2 b)$. Exercise training did not significantly affect total cholesterol, LDL-C or HDL-C in both, RESP and NON-RESP (table 1).

Exercise training had no time $(p=0.252)$, group $(p=0.417)$ or time*group $(p=0.118)$ effect on fasting FFA levels (table 3). Exercise training induced a significant time*group $(p=0.042)$, but no time $(p=0.981)$ or group $(p=0.356)$ effect for insulinmediated suppression of lipolysis (table 3 ). RESP tended to increase their ability to suppress lipolysis from $81.1 \pm 1.0$ to $82.9 \pm 0.9 \%(p=0.051)$, whereas insulinstimulated suppression of lipolysis in NON-RESP was unaffected with exercise training (from $80.2 \pm 1.9$ to $78.3 \pm 2.4 \%, p=0.194$ ). Individual responses to exercise training on insulin-stimulated suppression of lipolysis ( $\triangle F F A \%)$ are depicted in figure $1 \mathrm{~h}$. In the complete group of subjects, exercise training induced changes $\triangle \mathrm{GIR}$ did not significantly correlate with $\triangle$ FFA $\%(r=-0.225, p=0.063)$.

\section{CHANGES IN SKELETAL MUSCLE AND HEPATIC INSULIN SENSITIVITY}

In line with the stratification design on $\Delta G I R$, there was a significant time*group $(p<0.001)$, but no time $(p=0.222)$ or group $(p=0.378)$ effect for delta $R_{d}$ upon exercise training. Thus, upon stratification for the exercise training mediated change in whole body glucose uptake also delta $R_{d}$ responded significantly different to exercise training in the RESP group compared to the NON-RESP group. RESP significantly increased delta $R_{d}$ with $29.3 \%$ (from $12.3 \pm 1.4$ to $15.9 \pm 1.4$ $\mu \mathrm{mol} / \mathrm{min} / \mathrm{kg}, \mathrm{p}<0.001$ ), while delta $R_{d}$ in NON-RESP did not significantly change upon exercise training (from $12.2 \pm 2.4$ to $9.8 \pm 2.9 \mu \mathrm{mol} / \mathrm{min} / \mathrm{kg}, \mathrm{p}=0.111$ ) (table $3)$. As skeletal muscle represents approximately $80 \%$ of the whole body insulinstimulated glucose uptake, these results were anticipated upon stratification in RESP and NON-RESP based on $\Delta G I R$. Individual responses for $\Delta R_{d}$ are represented 
in figure 3b. We next evaluated training effects on hepatic insulin sensitivity. Suppression of hepatic glucose output by insulin did not show a time $(p=0.184)$ or group ( $p=0.901$ ) effect with exercise training. There was a significant time*group $(p=0.006)$ effect, in which RESP improved their insulin-stimulated suppression of EGP (from $-82.1 \pm 6.5$ to $-97.4 \pm 5.3 \%, p<0.001$ ), indicating improved hepatic insulin sensitivity in the RESP group, whereas no improvement could be detected in the NON-RESP subjects. If anything, hepatic insulin sensitivity was negatively affected by the exercise training program in the NON-RESP group (insulin-stimulated suppression of EGP changed from $-91.1 \pm 7.0$ to $-85.6 \pm 4.4 \%, p=0.456$ after training) (table 3 ). Individual responses in hepatic insulin sensitivity are depicted in figure 3c.

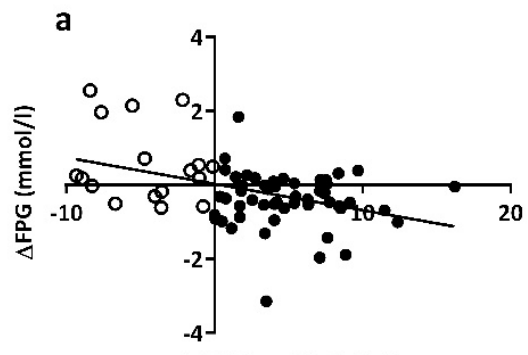

$\Delta \mathrm{GIR}(\mu \mathrm{mol} / \mathrm{min} / \mathrm{kg})$
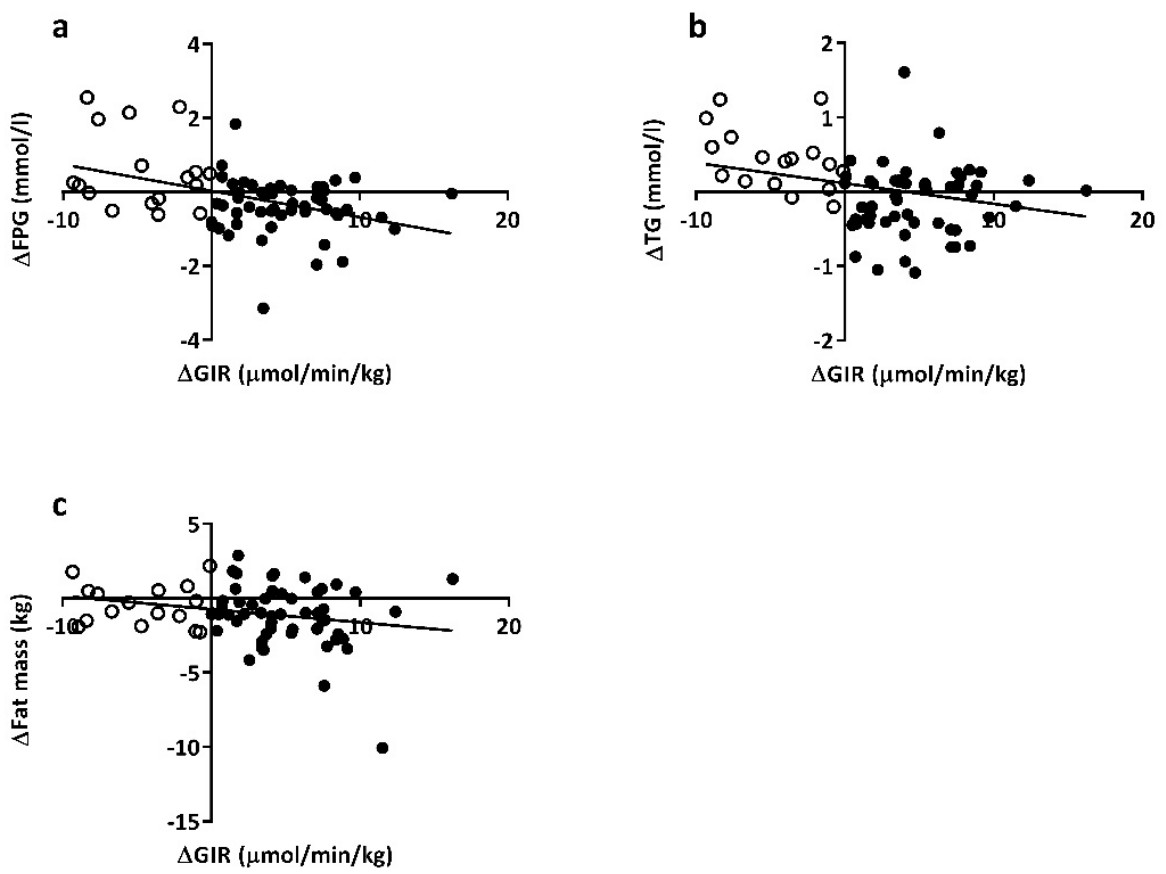

Figure 2. Comparison between the change in glucose infusion rate upon exercise training $(\Delta G I R)$ and exercise training induced changes in (a) fasting plasma glucose ( $\triangle F P G, n=71)$, (b) fasting triglycerides $(\Delta T G, n=71)$, (c) fat mass ( $\Delta$ fat mass, $n=70)$. $\Delta G I R$ negatively correlated with $\Delta F G P(r=-0.415, p<0.001), \Delta T G(r=-0.371, p=0.001)$ and $\Delta$ fat mass $(r=-0.237, p=0.048)$. Open dots represent NON-RESP, black dots represent RESP. 


\section{DISCUSSION}

Physical exercise training is a cornerstone in the prevention and treatment of type 2 diabetes mellitus ${ }^{1}$. Whereas the general assumption is that regular exercise training improves insulin sensitivity in all subjects compliant to a training program, recent data also identified non-responders to the insulin sensitizing effect of regular physical exercise ${ }^{22}$. We aimed to identify baseline characteristics and evaluated the impact of exercise training in responders and non-responders based upon improvements in whole body insulin sensitivity upon exercise training.

Upon stratification to changes in whole body insulin sensitivity following exercise training we observed that approximately $24 \%$ of the subjects who were compliant to exercise training, failed to improve whole body insulin sensitivity upon a 12 week tightly controlled, progressive, combined aerobic and resistance exercise training program. In these so-called non-responders (NON-RESP), the glucose infusion rate required to maintain euglycemia during a hyperinsulinemic-euglycemic clamp before training was higher than after training (reflected in a negative $\Delta G I R$, indicative of a drop in whole body insulin sensitivity). In fact, the maximal improvement in $\Delta \mathrm{GIR}$ in the subject most responsive to the exercise training program $(\Delta G I R=+40 \%)$ was in the same order of magnitude as the maximal drop in $\Delta G I R$ in the non-responsive subject $(\Delta G I R=-52 \%)$. This difference in benefit could not be attributed to differences in adherence to the exercise training program (98.1 $\pm 0.5 \%$ in RESP and $96.8 \pm 1.2 \%$ in NON-RESP, $p=0.245$ ); and classical parameters of physical fitness and muscle strength, including skeletal muscle mitochondrial mass, increased similarly in RESP and in NON-RESP. Moreover, we could not identify any differences in baseline parameters, which could be at the base of the differential response in $\Delta G I R$ to exercise training.

While the health benefits of an exercise training program in middle-aged overweight/obese people are generally not debated, the outcome on clinically relevant parameters like FPG and plasma TG levels is inconsistent ${ }^{22}$. Interestingly, we observed in the RESP group that plasma TG levels tended to drop after exercise training, whereas in the NON-RESP group plasma TG levels rose post-training. Similar observations were made for FPG; in the RESP group we observed the anticipated drop in FPG, whereas in the NON-RESP FPG levels actually rose. Plasma 
TG are transported in the form of lipoproteins, and changes in plasma TG levels in the fasted state are predominantly due to changes in hepatic very low density lipoprotein (VLDL)-TG secretion or changes in clearance of plasma TG, incorporated in LDL-C particles, by skeletal muscle ${ }^{23}$. As we did not observe changes in LDL-C upon exercise training in neither group, the alterations in plasma TG most likely originate from changes in hepatic VLDL-TG secretion. Thus, exercise training may have had a lowering effect on hepatic VLDL-TG secretion in the RESP group, while in the NON-RESP group VLDL-TG secretion by the liver most likely increased upon exercise training, actually reflecting more severe hepatic insulin resistance. Elevated VLDL-TG secretion is more common under conditions of severe insulin resistance ${ }^{24}$, and hence matches with the observation in the NON-RESP group that not only whole body insulin sensitivity deteriorated upon exercise training, but also hepatic insulin sensitivity was more compromised upon exercise training, although not significantly. We have to mention that we measured hepatic insulin sensitivity upon high-dose insulin infusion $\left(40 \mathrm{mU} / \mathrm{m}^{2} / \mathrm{min}\right)$. Ideally, suppression of EGP is calculated upon low-dose insulin infusion $\left(10 \mathrm{mU} / \mathrm{m}^{2} / \mathrm{min}\right)$, and therefore, our observation should be confirmed using low-dose insulin infusion in the future.

Importantly, the observation that FPG only dropped significantly after exercise training in the RESP group and not in the total group (RESP and NON-RESP together) helps to understand the commonly modest effects of exercise training on $\mathrm{FPG}^{25}$. Especially since the approximately $24 \%$ of non-responsiveness in the present study is similar to numbers of non-responders in other exercise training studies ${ }^{5}$. Thus, it could be that also in other studies exercise training lowering effects on FPG are limited to the individuals who improve insulin sensitivity upon exercise training. Therefore, it would be of interest to see if stratification of these studies in responders and non-responders would also result in significant FPG lowering effects in the responders only.

As anticipated, the exercise training program resulted in increased physical fitness as reflected by improvements in $\mathrm{VO}_{2 \max }, \mathrm{W}_{\max }$ and $1 \mathrm{RM}$. The increase was similar for RESP and NON-RESP. Thus, in the NON-RESP group, improved physical fitness dissociates from the change in whole body insulin sensitivity. This dissociation is remarkable as improvements in insulin sensitivity following exercise training are often associated with improvements in physical fitness ${ }^{26}$. It has, however, 
previously been suggested that physical activity could influence cardiorespiratory fitness and insulin sensitivity by independent parallel mechanisms ${ }^{4,26-29}$. Likewise, it has been shown that people with T2DM who do not possess a clinically meaningful improvement in $\mathrm{VO}_{2 \text { peak }}$ may nevertheless display significant improvements in $\mathrm{HbA}_{1 \mathrm{c}}$ and body fat, to an extent that is similar to improvements observed in $\mathrm{VO}_{2 \text { peak }}$-responders ${ }^{4}$. Although we basically report the reverse - almost all subjects improved markers for physical fitness whereas only a subset also improved insulin sensitivity - our study is in support of the idea that there might be no direct link between improvement in physical fitness and insulin sensitivity.
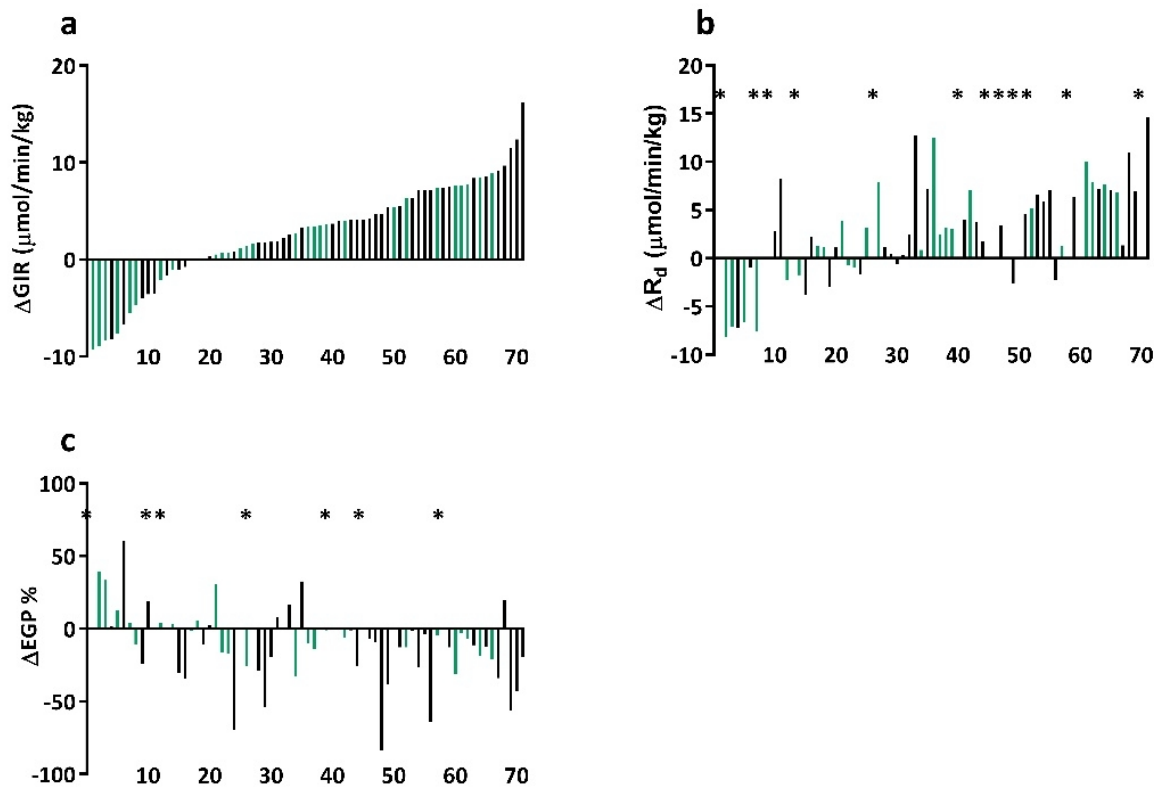

Figure 3. Individual data on changes in (a) glucose infusion rate $(\Delta G \mid R)$ during 40 $\mathrm{mU} / \mathrm{m}^{2} / \mathrm{min}$ and corresponding changes in (b) insulin-stimulated glucose disposal $\left(\Delta R_{d}\right)$ and (c) insulin-stimulated suppression of endogenous glucose disposal ( $\triangle E G P \%$ ). Green columns represent T2DM patients, black columns non-T2DM subjects. * represents missing data. 
Previous studies that observed dissociation between exercise training mediated improvements in physical fitness and insulin sensitivity have identified exercise associated weight loss ${ }^{11,12}$, blunted induction of mitochondrial biogenesis ${ }^{30,31}$ or improved resting in vivo mitochondrial function ${ }^{32}$ as contributors to the lack of effect of exercise training on insulin sensitivity. Although we did not examine these factors systematically, it is of interest to note that we indeed observed (upon examination of the complete group) that those who lost most fat mass upon exercise training were those who improved most in insulin sensitivity. Thus, it could be suggested that indeed the ability of losing body fat upon exercise training is a determinant for responsiveness of insulin sensitivity to exercise training.

As a crude marker of mitochondrial biogenesis and mitochondrial function, we measured protein content of 5 distinct structural proteins in the electron transport chain. We observed that this marker increased in almost all subjects and in a comparable fashion in RESP and NON-RESP. We previously demonstrated that exercise training increased mitochondrial content to a similar extent in obese control subjects and T2DM patients ${ }^{33}$. In our previous study, the concomitant improvement in mitochondrial oxidative capacity was mainly accounted for by the exercise training induced increase in mitochondrial content, and not intrinsic mitochondrial capacity. Interestingly, our current observation that mitochondrial content increased similarly in NON-RESP and RESP along with the lack of correlation between the changes on OXPHOS content and glucose infusion rate, may suggest that insulin sensitivity and mitochondrial content/function are exercise training mediated responses that are not necessarily causally related. Phielix et al. ${ }^{33}$ examined the association between mitochondrial function and insulin sensitivity. They found no correlation between (changes in) insulin sensitivity and (changes in) mitochondrial function, although both improved in parallel upon exercise training.

Genetic and epigenetic factors dictating the myocellular adaptation to exercise training have also been mentioned as modulating factors for the response of insulin sensitivity to exercise training. Likewise, a 29-gene molecular signature has been used to predict exercise training mediated improvements in $\mathrm{VO}_{2 \max }{ }^{34}$. In the present study most subjects did improve $\mathrm{VO}_{2 \max }$, also those who were in the NON-RESP group. Therefore, it is unlikely that this genetic signature explains the observed non-responsiveness in insulin sensitivity in the present study. Along the same lines, 
a single nucleotide polymorphism ( $\mathrm{rs} 540467 \mathrm{G} / \mathrm{A}$ ) in the gene encoding for NDUFB6 (one of the subunits of complex I of the electron transport chain) was shown to affect mitochondrial function and has hence been classified as a 'non-responder gene' ${ }^{32}$. It should be noted though that in that particular study whole body insulin sensitivity has not been measured and therefore it is not clear if this gene defect affects exercise training mediated changes in insulin sensitivity is. Detailed genetic analysis should be performed to elucidate if indeed genetic and/or epigenetic factors contribute to the non-responsiveness to training we reported here.

In conclusion, we here report that in this group of middle-aged overweight/obese and T2DM men, $23.9 \%$ failed to respond to a 12 week exercise training program with respect to whole body insulin sensitivity. Classical parameters of physical fitness and muscle strength, including skeletal muscle mitochondrial mass, increased similarly in responders and non-responders. We could not identify any differences in baseline characteristic, which could be at the base of the differential response to exercise training. Most strikingly, FPG and plasma TG levels decreased in the RESP group after training, whereas both increased in the NON-RESP group. Thus, a positive response on whole body insulin sensitivity upon exercise training seems to be necessary to improve FPG and plasma TG levels. Importantly, the mechanism underlying this differential response remains to be elucidated. Therefore, more research is warranted to understand why some people do, and other do not, improve insulin sensitivity upon exercise training. 


\section{REFERENCES}

1. Standards of medical care in diabetes--2015: summary of revisions. Diabetes Care 38 Suppl, S4 (2015).

2. Boule, N.G., Haddad, E., Kenny, G.P., Wells, G.A. \& Sigal, R.J. Effects of exercise on glycemic control and body mass in type 2 diabetes mellitus: a meta-analysis of controlled clinical trials. Jama 286, 1218-1227 (2001).

3. Organization, W.H. Global Recommendations on Physical Activity for Health. in Global Recommendations on Physical Activity for Health (Geneva, 2010).

4. Pandey, A., et al. Metabolic Effects of Exercise Training Among FitnessNonresponsive Patients With Type 2 Diabetes: The HART-D Study. Diabetes Care 38, 1494-1501 (2015).

5. Stephens, N.A. \& Sparks, L.M. Resistance to the beneficial effects of exercise in type 2 diabetes: are some individuals programmed to fail? The Journal of clinical endocrinology and metabolism 100, 43-52 (2015).

6. Bouchard, C., et al. Adverse metabolic response to regular exercise: is it a rare or common occurrence? PLoS One 7, e37887 (2012).

7. Slentz, C.A., Houmard, J.A. \& Kraus, W.E. Exercise, abdominal obesity, skeletal muscle, and metabolic risk: evidence for a dose response. Obesity 17 Suppl 3, S27-33 (2009).

8. Marzolini, S., Oh, P.I. \& Brooks, D. Effect of combined aerobic and resistance training versus aerobic training alone in individuals with coronary artery disease: a meta-analysis. Eur J Prev Cardiol 19, 81-94 (2012).

9. Church, T.S., et al. Effects of aerobic and resistance training on hemoglobin A1c levels in patients with type 2 diabetes: a randomized controlled trial. Jama 304, 2253-2262 (2010).

10. Stephens, N.A., et al. A transcriptional signature of "exercise resistance" in skeletal muscle of individuals with type 2 diabetes mellitus. Metabolism (2015).

11. Knowler, W.C., et al. Reduction in the incidence of type 2 diabetes with lifestyle intervention or metformin. N Engl J Med 346, 393-403 (2002). 
12. Tuomilehto, J., et al. Prevention of type 2 diabetes mellitus by changes in lifestyle among subjects with impaired glucose tolerance. N Engl J Med 344, 1343-1350 (2001).

13. Meex, R.C., et al. Restoration of muscle mitochondrial function and metabolic flexibility in type 2 diabetes by exercise training is paralleled by increased myocellular fat storage and improved insulin sensitivity. Diabetes 59, 572-579 (2010).

14. Brouwers, B., Schrauwen-Hinderling, V.B., Gemmink, A., Havekes, B., Bruls, Y., Dahlmans, D., Hesselink, M.K.C., Schrauwen, P. Exercise training reduces liver fat content and improves peripheral, but not hepatic insulin sensitivity in overweight and obese, middle-aged men with non-alcoholic fatty liver disease. in prep.

15. Kuipers, H., Verstappen, F.T., Keizer, H.A., Geurten, P. \& van Kranenburg, G. Variability of aerobic performance in the laboratory and its physiologic correlates. Int J Sports Med 6, 197-201 (1985).

16. Siri, W.E. The gross composition of the body. Adv Biol Med Phys 4, 239-280 (1956).

17. Mensink, M., Blaak, E.E., van Baak, M.A., Wagenmakers, A.J. \& Saris, W.H. Plasma free Fatty Acid uptake and oxidation are already diminished in subjects at high risk for developing type 2 diabetes. Diabetes 50, 2548-2554 (2001).

18. Ackermans, M.T., et al. The quantification of gluconeogenesis in healthy men by (2) $\mathrm{H} 2 \mathrm{O}$ and [2-(13)C]glycerol yields different results: rates of gluconeogenesis in healthy men measured with (2) $\mathrm{H} 2 \mathrm{O}$ are higher than those measured with [2-(13)C]glycerol. J Clin Endocrinol Metab 86, 22202226 (2001).

19. Steele, R. Influences of glucose loading and of injected insulin on hepatic glucose output. Ann N Y Acad Sci 82, 420-430 (1959).

20. Friedewald, W.T., Levy, R.I. \& Fredrickson, D.S. Estimation of the concentration of low-density lipoprotein cholesterol in plasma, without use of the preparative ultracentrifuge. Clin Chem 18, 499-502 (1972).

21. Bergstrom, J., Hermansen, L., Hultman, E. \& Saltin, B. Diet, muscle glycogen and physical performance. Acta Physiol Scand 71, 140-150 (1967).

22. van de Weijer, T., et al. Relationships between mitochondrial function and metabolic flexibility in type 2 diabetes mellitus. PloS one 8, e51648 (2013). 
23. Ramasamy, I. Recent advances in physiological lipoprotein metabolism. Clin Chem Lab Med 52, 1695-1727 (2014).

24. Choi, S.H. \& Ginsberg, H.N. Increased very low density lipoprotein (VLDL) secretion, hepatic steatosis, and insulin resistance. Trends Endocrinol Metab 22, 353-363 (2011).

25. Cornelissen, V.A., Fagard, R.H., Coeckelberghs, E. \& Vanhees, L. Impact of resistance training on blood pressure and other cardiovascular risk factors: a meta-analysis of randomized, controlled trials. Hypertension 58, 950-958 (2011).

26. Messier, V., Malita, F.M., Rabasa-Lhoret, R., Brochu, M. \& Karelis, A.D. Association of cardiorespiratory fitness with insulin sensitivity in overweight and obese postmenopausal women: a Montreal Ottawa New Emerging Team study. Metabolism 57, 1293-1298 (2008).

27. Heikkila, H.M., Krachler, B., Rauramaa, R. \& Schwab, U.S. Diet, insulin secretion and insulin sensitivity--the Dose-Responses to Exercise Training (DR's EXTRA) Study (ISRCTN45977199). Br J Nutr 112, 1530-1541 (2014).

28. Brennan, A.M., Lam, M., Stotz, P., Hudson, R. \& Ross, R. Exercise-induced improvement in insulin sensitivity is not mediated by change in cardiorespiratory fitness. Diabetes Care 37, e95-97 (2014).

29. Vollaard, N.B., et al. Systematic analysis of adaptations in aerobic capacity and submaximal energy metabolism provides a unique insight into determinants of human aerobic performance. J Appl Physiol (1985) 106, 1479-1486 (2009).

30. Kacerovsky-Bielesz, G., et al. Short-term exercise training does not stimulate skeletal muscle ATP synthesis in relatives of humans with type 2 diabetes. Diabetes 58, 1333-1341 (2009).

31. De Filippis, E., et al. Insulin-resistant muscle is exercise resistant: evidence for reduced response of nuclear-encoded mitochondrial genes to exercise. Am J Physiol Endocrinol Metab 294, E607-614 (2008).

32. Kacerovsky-Bielesz, G., et al. A single nucleotide polymorphism associates with the response of muscle ATP synthesis to long-term exercise training in relatives of type 2 diabetic humans. Diabetes Care 35, 350-357 (2012).

33. Phielix, E., Meex, R., Moonen-Kornips, E., Hesselink, M.K. \& Schrauwen, P. Exercise training increases mitochondrial content and ex vivo 
mitochondrial function similarly in patients with type 2 diabetes and in control individuals. Diabetologia 53, 1714-1721 (2010).

34. Timmons, J.A. Variability in training-induced skeletal muscle adaptation. J Appl Physiol (1985) 110, 846-853 (2011). 




\section{CHAPTER 9}

GENERAL DISCUSSION 
The prevalence of obesity is reaching epidemic proportions worldwide ${ }^{1}$. Obesity is associated with type 2 diabetes mellitus (T2DM) ${ }^{2}$, and both obesity and T2DM are associated with insulin resistance ${ }^{3}$. The development of insulin resistance often coincides with fat accumulation in peripheral tissues ${ }^{4,5}$. In the liver, this is referred to as non-alcoholic fatty liver (NAFL). NAFL is closely related to insulin resistant states, such as obesity, the metabolic syndrome and $\mathrm{T}^{2} \mathrm{DM}^{6-9}$. Exercise is considered fundamental in the treatment and prevention of $\mathrm{T}^{2} \mathrm{DM}^{10}$ and may also have beneficial metabolic effects on liver fat metabolism. The research performed in this $\mathrm{PhD}$ thesis focused on the impact exercise has as a therapeutic tool on modulating liver fat metabolism, insulin resistance and cardiovascular risk factors associated with NAFL and T2DM.

\section{IS NON-ALCOHOLIC FATTY LIVER A PREDIABETIC STATE?}

T2DM is characterized by a sustained reduction in insulin sensitivity. Progression from the early stages of insulin resistance (when glycemic control is not yet abnormal) to overt T2DM is a gradual and underdiagnosed process ${ }^{11}$. In this process the distinction is made between normal glucose tolerance (NGT), impaired glucose tolerance (IGT), impaired fasting glucose (IFG) and overt T2DM ${ }^{12}$. The prevalence of IGT and IFT is significantly higher in people with non-alcoholic fatty liver (NAFL) than in people with normal intrahepatic lipid $(\mathrm{IHL})$ content $^{6,7}$. Furthermore, having a fatty liver increases the risk to develop T2DM by 2 -fold in comparison to non-NAFL subjects, when adjusted for age, gender, race and $\mathrm{BMI}^{13,14}$. Ortiz-Lopez et al. ${ }^{15}$ showed that in a group of 118 overweight and obese patients with NAFL, $85 \%$ was diagnosed with IGT, IFT or T2DM, while this was only $30 \%$ in the non-NAFL control group. In turn, people with T2DM are more prone to have a fatty liver, and the prevalence of NAFL might be as high as $50-75 \%$ in T2DM patients ${ }^{6,8,16}$. Thus, it is clear that NAFL and T2DM frequently co-exist.

With T2DM, insulin sensitivity of liver, skeletal muscle and adipose tissue is significantly reduced ${ }^{17,18}$. Also in people with NAFL impaired hepatic and whole body insulin sensitivity in comparison to non-NAFL individuals has been previously described ${ }^{19-22}$. In obese middle-aged subjects it has been shown that people with high IHL content had markedly hepatic, adipose tissue and skeletal muscle insulin 
resistance in comparison to people with low $\mathrm{IHL}$ content ${ }^{19}$. In overweight ${ }^{21}$ and in obese $^{22}$ younger adults, having NAFL was related to impaired insulin sensitivity in comparison to BMI-matched adolescents without NAFL. In chapter 3, we investigated the severity of the metabolic disturbances in NAFL compared to the metabolic disturbances observed in T2DM. We observed that under conditions of insulin stimulation hepatic, skeletal muscle and adipose tissue insulin sensitivity all are impaired in NAFL subjects to a similar degree as in patients with overt T2DM. This observation was not completely anticipated. We expected to find intermediate values of insulin resistance in NAFL subjects in accordance with previous observations in other populations at risk to develop T2DM. In first degree relatives for example, insulin-stimulated glucose disposal was lower compared to control subjects, but was still higher than in T2DM patients ${ }^{17}$. In subjects with IGT, insulinstimulated glucose disposal was $34 \%$ lower than in NGT subjects, but $40 \%$ higher than in T2DM patients ${ }^{23}$. Thus, our observations point out that insulin resistance in NAFL patients is more severe than in other groups known to be at risk to develop T2DM and in fact should already be considered a target of intervention.

We further observed that NAFL subjects had marked fasting hyperinsulinemia, while fasting plasma glucose concentrations were comparable to non-NAFL subjects (chapter 3 ). The presence of hyperinsulinemia to compensate for impaired insulin sensitivity, and thereby maintaining normoglycemia, has been frequently reported in previous studies with NAFL subjects ${ }^{19,21,22}$. It might be that higher fasting insulin concentrations arise from increased insulin production to compensate for insulin resistance in NAFL. We did not measure pancreatic $\beta$-cell function, but a possible marker for this would be the measurement of serum Cpeptide concentrations ${ }^{24}$. While insulin is exposed to first-pass hepatic extraction after secretion, C-peptide passes through the liver without extraction, and thus plasma C-peptide concentrations reflect pancreatic insulin secretion ${ }^{25}$. Previous studies have shown that a fatty liver strongly associates with fasting C-peptide concentrations ${ }^{24}$. In non-diabetic subjects, Kotronen et al. ${ }^{26}$ reported that the best correlates of $\mathrm{IHL}$ content were fasting insulin and fasting C-peptide concentrations. Perseghin et al. ${ }^{25}$ found that individuals with NAFL were characterized by enhanced fasting pancreatic $\beta$-cell function when compared to non-NAFL subjects. Thus, elevated insulin levels due to elevated pancreatic $\beta$-cell function may suffice to mask severe deteriorations in glucose metabolism that are already present in NAFL. 
It can be hypothesized that once insulin production also fails to compensate for the observed insulin resistance, these NAFL individuals eventually develop T2DM.

Exercise training is a recognized cornerstone in the treatment of insulin resistance and T2DM. If NAFL is a risk factor to develop T2DM, exercise training might be beneficial to alleviate the metabolic disturbances. Previously, a significant improvement in whole body insulin sensitivity measured by HOMA-IR after 8 weeks of resistance exercise in NAFL subjects has been reported by Hallsworth et al. ${ }^{27}$, whereas Johnson et al. ${ }^{28}$ did not observe an improvement in HOMA-IR after 4 weeks of aerobic exercise training. No previous study, however, investigated the tissue specific effects of exercise training on insulin sensitivity in NAFL. In this thesis, we found that 12 weeks of exercise training positively affected skeletal muscle insulin sensitivity, also in NAFL subjects (chapter 4). Our finding is in agreement with previous data in other diabetes prone populations like first degree relatives ${ }^{29}$, people with IFT or IGT ${ }^{30}$ as well as in patients with full-blown $\mathrm{T}^{2} \mathrm{DM}^{18}$, who all are capable of improving insulin-stimulated glucose uptake upon exercise training.

Taken together, people with NAFL are characterized by severe insulin resistance comparable to T2DM patients. A preserved pancreatic $\beta$-cell function might initially compensate for the metabolic aberrations in the fasted state, as suggested by the presence of hyperinsulinemia. However, over time, pancreatic $\beta$-cell function might fail, and eventually hyperglycemia and T2DM may develop. Exercise training effectively improves the metabolic profile of people with NAFL to prevent the possible development towards type 2 diabetes mellitus. 


\section{DOES EXERCISE POSITIVELY AFFECT LIVER METABOLISM?}

\section{INTRAHEPATIC LIPID CONTENT}

Lifestyle interventions are considered a cornerstone for treatment of NAFL ${ }^{31}$ and cross-sectional studies have shown that higher levels of physical activity are associated with lower levels of IHL content ${ }^{32,33}$. However, only few studies have investigated the direct effect of exercise training on $\mathrm{IHL}$ content in interventional studies ${ }^{27,28,31,34-36}$. In this thesis, we investigated whether 12 weeks of supervised, combined aerobic and resistance exercise training without targeted weight loss could decrease IHL content in non-NAFL control subjects, NAFL subjects and T2DM patients (chapter 5). We found a significant decrease in IHL content in the NAFL subjects. Intriguingly, no change in IHL content was present in the non-NAFL control subjects and T2DM patients. This is the first study to investigate the effect of exercise training on $\mathrm{IHL}$ content in these 3 populations in a controlled parallel design, all performing exercise training at a comparable intensity. Therefore we were able to conclude that exercise training is especially effective in decreasing $\mathrm{IHL}$ content in those possessing high liver fat concentrations. This observation is in agreement with previous studies which included only one group of subjects (nonNAFL, NAFL or T2DM). Indeed aerobic and resistance exercise training have been described to be effective in lowering $\mathrm{IHL}$ content in people with NAFL $27,28,31,35,36$. In non-NAFL obese subjects one study observed a significant decrease in $\mathrm{IHL}$ content ${ }^{34}$, while 2 other studies were not able to measure differences in liver fat content upon exercise training ${ }^{37,38}$. Bacchi et al. ${ }^{31}$ found lower liver fat content in T2DM patients after 4 months of aerobic and resistance exercise training. However, in their study only T2DM patients with NAFL were included, while our T2DM patients were not selected for having a fatty liver.

The long-term exercise training effect might be explained by the accumulated effect of acute exercise bouts. Insulin sensitivity, for example, already improves after a single exercise bout ${ }^{39}$. In chapter 4 , we investigated whether an acute bout of exercise could lower IHL content. We found that IHL content did not decrease after an acute exercise bout in middle-aged overweight men with a wide range of $\mathrm{IHL}$ 
content. Our observation that acute exercise does not lower IHL content is supported by studies in healthy normal-weight participants ${ }^{40,41}$ and in trained athletes ${ }^{42}$. Moreover, we found that, in the post-exercise recovery phase, IHL content was increased at $4 \mathrm{~h}$ post-exercise in the fasted condition. The increase in $\mathrm{IHL}$ content might be explained by higher availability of plasma free fatty acids (FFA) to the liver. During exercise in the fasted state, plasma FFA profoundly increases ${ }^{43}$. The circulatory fatty acids might be taken up by the liver, resynthesized to triglycerides (TG) and eventually increase IHL content. This is supported by our own finding, in which the increase in IHL content was absent when subjects consumed a glucose beverage during and after exercise, to lower the plasma FFA concentrations.

Thus, exercise training has the potential to lower IHL content, especially in people with high liver fat concentrations. Contrary, acute exercise does not decrease IHL content. Therefore, the effect of exercise training on liver fat content cannot simply be explained by changes upon acute exercise.

\section{HEPATIC ENERGY STATUS}

Hepatic ATP levels have been described to be lower in insulin resistance and T2DM $^{44,45}$. Moreover, YATP concentrations ${ }^{44}$ and hepatocellular ATP production ${ }^{45}$ negatively correlate to IHL content. Furthermore, hepatic ATP recovery after ATP depletion is significantly impaired in non-alcoholic steatohepatitis (NASH) compared to healthy individuals ${ }^{46}$. Mitochondria are the main site of aerobic ATP production, and mitochondrial dysfunction has been reported in skeletal muscle of pre-diabetic and diabetic subjects. Upon exercise training, skeletal muscle mitochondrial function has been shown to be restored ${ }^{18}$. Thus, one may hypothesize that mitochondrial dysfunction in liver may associate with hepatic insulin resistance at baseline and that exercise training mediated improvements in liver insulin sensitivity may be paralleled by improvement in hepatic mitochondrial function. Therefore, we also investigated if exercise resulted in changes in hepatic ATP concentration, a possible marker for hepatic mitochondrial function. 
Concomitant with the increase in IHL content after acute exercise in the fasted state we observed a trend towards a decreased hepatic energy status in middle-aged overweight men with a wide range of IHL content (chapter 4). A possible explanation could be that a decrease in hepatic ATP concentration is a direct consequence of acute exercise, due to increased energy demand. A study in mice, for example, showed that after an acute exercise bout hepatic ATP was significantly decreased, while AMP concentration was increased ${ }^{47}$. Though, we did not observe a decreased ATP/Total Phosphorus ratio after acute exercise in the glucosesupplemented condition. This could suggest that the increase in IHL content after acute exercise in the fasted state, rather than the exercise bout per se, influenced hepatic energy status. Thus, IHL accumulation could be an important determinant in the impaired hepatic energy status seen in T2DM and NASH. However, Schmid et al. ${ }^{45}$ detected a clear difference in flux of ATP between T2DM and non-diabetic subjects despite comparable IHL content, arguing hepatic insulin resistance is a more important factor explaining lower hepatic energy status. In chapter 3 of this thesis, ATP/Total Phosphorus ratio was comparable in non-NAFL subjects, NAFL subjects and T2DM patients, although both IHL content and hepatic insulin resistance was lower in non-NAFL subjects than in the other 2 groups. Since we used ATP/Total Phosphorus ratio, we could not detect parallel increases or decreases of phosphor metabolites, as have been described in alcoholic fatty liver ${ }^{48}$. Therefore we cannot conclude on potential differences in absolute ATP concentrations or flux of ATP concentrations.

Szendroedi et al. ${ }^{44}$ previously reported that hepatic YATP negatively associated with hepatic insulin sensitivity, but not with skeletal muscle insulin sensitivity. The suggestion that hepatic insulin resistance could be an important determinant for hepatic energy status might explain why hepatic energy status was unaffected upon exercise training. In chapter 5 , we found that exercise training significantly increased peripheral, but not hepatic insulin sensitivity over time. Furthermore in NAFL subjects, the significant reduction in IHL content upon exercise training was accompanied by improved peripheral insulin sensitivity, but did not result in higher hepatic insulin sensitivity or higher hepatic energy status. Thus, the lack of effect of exercise training on hepatic energy status coincided with a lack of effect on hepatic insulin sensitivity. This might suggest that an effect on hepatic insulin sensitivity may be required to affect hepatic energy status. 


\section{DETERMINANTS OF DIVERGENT RESPONSES TO EXERCISE TRAINING}

Exercise training has been shown to improve insulin sensitivity and glycemic control $^{18,49}$. Intriguingly, despite the known favorable effects of exercise training, not all individuals do indeed benefit and some people respond poorly to training with respect to metabolic parameters ${ }^{50,51}$. In chapter 8 , we examined differences in baseline characteristics and the impact of exercise training on metabolic parameters in obese subjects and T2DM patients, who were categorized as responders or non-responders based upon exercise training induced changes in whole body insulin sensitivity. There were no differences in baseline characteristics between responders and non-responders. Moreover, upon exercise training maximal aerobic capacity $\left(\mathrm{VO}_{2 \max }\right)$, maximal work load $\left(\mathrm{W}_{\max }\right)$ and muscle strength improved to a similar extend in both groups. Thus, the divergent exercise training response on insulin sensitivity between responders and non-responders could not be explained by a different exercise training response on cardiorespiratory fitness. Previous studies have shown that a smaller improvement in aerobic capacity upon exercise training does not necessarily coincides with a smaller improvement in metabolic parameters, such as $\mathrm{HbA}_{1 c}$ and insulin sensitivity ${ }^{51-53}$. These studies suggested that physical activity may influence cardiorespiratory fitness and insulin sensitivity by independent mechanisms.

Endurance-trained individuals are characterized by low fasting plasma glucose (FPG) and plasma TG concentrations. However, there is debate whether exercise training decreases FPG and TG. Cornelissen et $a .^{54}$ found that resistance exercise training significantly decreases plasma TG concentrations, but not FPG in healthy adults. Another meta-analysis ${ }^{55}$ concluded that exercise training is not effective in lowering TG and FPG levels in adults with the metabolic syndrome. In chapter 8, we found that exercise training significantly decreased plasma TG, but not FPG over time. Intriguingly, however, changes in plasma TG and FPG upon exercise training were related to changes in whole body insulin sensitivity with exercise training. More specifically, we found that fasting plasma TG and FPG levels decreased after exercise training in responders, while non-responders in terms of whole body insulin sensitivity increased plasma TG and FPG concentrations. A divergent 
response in plasma TG upon exercise training has been previously described in a large cohort of 6 different exercise training studies ${ }^{56}$. In this cohort 1 out of 10 individuals increased plasma TG upon exercise training. This observation was consistent in all 6 studies, even though the age and health status of the subjects were very different and the exercise training interventions were quite heterogeneous. The relationship with changes in insulin sensitivity was not measured, however. Thus chapter 8 suggests that (positive/negative) exercise training responses on whole body insulin sensitivity are paralleled by (positive/negative) exercise training responses on fasting plasma TG and FPG, but we cannot conclude on a possible cause-and-effect relationship. Most likely, changes in insulin sensitivity resulted in concomitant changes in plasma TG and FPG concentrations. We found that exercise training improved hepatic insulin sensitivity in responders, but deteriorated in non-responders. Given that endogenous glucose production is the main determinant of plasma glucose in the fasted state, this could have contributed to the observed changes in FPG. Moreover, changes in plasma TG levels most likely originate from changes in hepatic very low density lipoprotein (VLDL)-TG secretion, especially in the absence of changes in LDL-C concentrations ${ }^{57}$. VLDL-TG secretion is known to positively correlate with hepatic insulin resistance ${ }^{58}$. Nevertheless, vice-versa, the changes in plasma TG and FPG upon exercise training might also contribute to the divergent exercise training effect on whole body insulin sensitivity in responders and non-responders.

\section{FUTURE PERSPECTIVES}

In this PhD thesis, we showed that people with NAFL are characterized by severe insulin resistance, comparable to that seen in T2DM. Because people with NAFL do not develop hyperglycemia yet, it might be suggested that NAFL is a pre-diabetic state. However, it remains to be elucidated whether IHL contributes to the development of insulin resistance, insulin resistance is responsible for the increase in IHL content, or whether both develop simultaneously. Future research should focus on the mechanisms by which insulin resistance and liver fat metabolism are related to each other. 
Exercise training decreased IHL content concomitant with an improvement in skeletal muscle, but not liver or adipose tissue, insulin sensitivity in people with NAFL. Skeletal muscle is identified as a secretory organ, releasing myokines to the periphery. These myokines can mediate interaction between skeletal muscle and other organs, like the liver. Some myokines are released after muscle contraction, and therefore may contribute to the positive metabolic effects upon exercise training ${ }^{59}$. Therefore, it can be interesting to further investigate the possible crosstalk between liver and skeletal muscle in relation to changes in liver metabolism and insulin resistance upon exercise training.

Although most people benefit from exercise training, we found that a significant number of individuals do not improve their metabolic health upon exercise training. These people were not characterized by baseline differences in metabolic and cardiorespiratory parameters, and the exercise training response on cardiorespiratory fitness was normal. Intriguingly, the divergent response in insulin sensitivity upon exercise training was paralleled by a different response in plasma TG and FPG concentrations with exercise training. Additional research is warranted to examine if the increases in plasma TG and FPG contribute to the nonresponsiveness of whole body insulin sensitivity to training, or whether it is a consequence of the lower whole body insulin sensitivity post-training. Furthermore, the challenge is now to investigate whether other baseline predictors and/or genetic components of non-responders can be identified to screen individuals at risk. 


\section{REFERENCES}

1. Ng, M., et al. Global, regional, and national prevalence of overweight and obesity in children and adults during 1980-2013: a systematic analysis for the Global Burden of Disease Study 2013. Lancet 384, 766-781 (2014).

2. Kahn, S.E., Hull, R.L. \& Utzschneider, K.M. Mechanisms linking obesity to insulin resistance and type 2 diabetes. Nature 444, 840-846 (2006).

3. Reaven, G.M. Banting lecture 1988. Role of insulin resistance in human disease. Diabetes 37, 1595-1607 (1988).

4. Bilet, L., et al. Exercise-induced modulation of cardiac lipid content in healthy lean young men. Basic Res Cardiol 106, 307-315 (2011).

5. Krssak, M. \& Roden, M. The role of lipid accumulation in liver and muscle for insulin resistance and type 2 diabetes mellitus in humans. Rev Endocr Metab Disord 5, 127-134 (2004).

6. Leite, N.C., Villela-Nogueira, C.A., Cardoso, C.R. \& Salles, G.F. Non-alcoholic fatty liver disease and diabetes: from physiopathological interplay to diagnosis and treatment. World J Gastroenterol 20, 8377-8392 (2014).

7. Yki-Jarvinen, $\mathrm{H}$. Liver fat in the pathogenesis of insulin resistance and type 2 diabetes. Dig Dis 28, 203-209 (2010).

8. Utzschneider, K.M. \& Kahn, S.E. Review: The role of insulin resistance in nonalcoholic fatty liver disease. J Clin Endocrinol Metab 91, 4753-4761 (2006).

9. Yun, J.W., et al. Abnormal glucose tolerance in young male patients with nonalcoholic fatty liver disease. Liver Int 29, 525-529 (2009).

10. Albright, A., et al. American College of Sports Medicine position stand. Exercise and type 2 diabetes. Med Sci Sports Exerc 32, 1345-1360 (2000).

11. Dunstan, D.W., et al. The rising prevalence of diabetes and impaired glucose tolerance: the Australian Diabetes, Obesity and Lifestyle Study. Diabetes Care 25, 829-834 (2002).

12. Gastaldelli, A. Role of beta-cell dysfunction, ectopic fat accumulation and insulin resistance in the pathogenesis of type 2 diabetes mellitus. Diabetes Res Clin Pract 93 Suppl 1, S60-65 (2011).

13. Musso, G., Gambino, R., Cassader, M. \& Pagano, G. Meta-analysis: natural history of non-alcoholic fatty liver disease (NAFLD) and diagnostic accuracy of non-invasive tests for liver disease severity. Ann Med 43, 617-649 (2011). 
14. Kotronen, A. \& Yki-Jarvinen, H. Fatty liver: a novel component of the metabolic syndrome. Arterioscler Thromb Vasc Biol 28, 27-38 (2008).

15. Ortiz-Lopez, C., et al. Prevalence of prediabetes and diabetes and metabolic profile of patients with nonalcoholic fatty liver disease (NAFLD). Diabetes care 35, 873-878 (2012).

16. Williams, C.D., et al. Prevalence of nonalcoholic fatty liver disease and nonalcoholic steatohepatitis among a largely middle-aged population utilizing ultrasound and liver biopsy: a prospective study. Gastroenterology 140, 124-131 (2011).

17. Phielix, E., et al. Lower intrinsic ADP-stimulated mitochondrial respiration underlies in vivo mitochondrial dysfunction in muscle of male type 2 diabetic patients. Diabetes 57, 2943-2949 (2008).

18. Meex, R.C., et al. Restoration of muscle mitochondrial function and metabolic flexibility in type 2 diabetes by exercise training is paralleled by increased myocellular fat storage and improved insulin sensitivity. Diabetes 59, 572-579 (2010).

19. Fabbrini, E., et al. Intrahepatic fat, not visceral fat, is linked with metabolic complications of obesity. Proc Natl Acad Sci U S A 106, 15430-15435 (2009).

20. Hwang, J.H., et al. Increased intrahepatic triglyceride is associated with peripheral insulin resistance: in vivo MR imaging and spectroscopy studies. Am J Physiol Endocrinol Metab 293, E1663-1669 (2007).

21. Deivanayagam, S., et al. Nonalcoholic fatty liver disease is associated with hepatic and skeletal muscle insulin resistance in overweight adolescents. Am J Clin Nutr 88, 257-262 (2008).

22. Lee, S., Rivera-Vega, M., Alsayed, H.M., Boesch, C. \& Libman, I. Metabolic inflexibility and insulin resistance in obese adolescents with non-alcoholic fatty liver disease. Pediatr Diabetes 16, 211-218 (2015).

23. Saad, M.F., et al. A comparison between the minimal model and the glucose clamp in the assessment of insulin sensitivity across the spectrum of glucose tolerance. Insulin Resistance Atherosclerosis Study. Diabetes 43, 1114-1121 (1994).

24. Lattuada, G., Ragogna, F. \& Perseghin, G. Why does NAFLD predict type 2 diabetes? Curr Diab Rep 11, 167-172 (2011). 
25. Perseghin, G., et al. Elevated fasting plasma C-peptide occurs in nondiabetic individuals with fatty liver, irrespective of insulin resistance. Diabet Med 26, 847-854 (2009).

26. Kotronen, A., Westerbacka, J., Bergholm, R., Pietilainen, K.H. \& YkiJarvinen, H. Liver fat in the metabolic syndrome. J Clin Endocrinol Metab 92, 3490-3497 (2007).

27. Hallsworth, K., et al. Resistance exercise reduces liver fat and its mediators in non-alcoholic fatty liver disease independent of weight loss. Gut 60, 1278-1283 (2011).

28. Johnson, N.A., et al. Aerobic exercise training reduces hepatic and visceral lipids in obese individuals without weight loss. Hepatology 50, 1105-1112 (2009).

29. Ostergard, T., et al. Impact of exercise training on insulin sensitivity, physical fitness, and muscle oxidative capacity in first-degree relatives of type 2 diabetic patients. American journal of physiology. Endocrinology and metabolism 290, E998-1005 (2006).

30. Malin, S.K., et al. Insulin sensitivity and metabolic flexibility following exercise training among different obese insulin-resistant phenotypes. American journal of physiology. Endocrinology and metabolism 305, E12921298 (2013).

31. Bacchi, E., et al. Both resistance training and aerobic training reduce hepatic fat content in type 2 diabetic subjects with nonalcoholic fatty liver disease (the RAED2 Randomized Trial). Hepatology 58, 1287-1295 (2013).

32. Perseghin, G., et al. Habitual physical activity is associated with intrahepatic fat content in humans. Diabetes Care 30, 683-688 (2007).

33. Kantartzis, K., et al. High cardiorespiratory fitness is an independent predictor of the reduction in liver fat during a lifestyle intervention in nonalcoholic fatty liver disease. Gut 58, 1281-1288 (2009).

34. Finucane, F.M., et al. The effects of aerobic exercise on metabolic risk, insulin sensitivity and intrahepatic lipid in healthy older people from the Hertfordshire Cohort Study: a randomised controlled trial. Diabetologia 53, 624-631 (2010).

35. Sullivan, S., Kirk, E.P., Mittendorfer, B., Patterson, B.W. \& Klein, S. Randomized trial of exercise effect on intrahepatic triglyceride content and 
lipid kinetics in nonalcoholic fatty liver disease. Hepatology 55, 1738-1745 (2012).

36. van der Heijden, G.J., et al. A 12-week aerobic exercise program reduces hepatic fat accumulation and insulin resistance in obese, Hispanic adolescents. Obesity (Silver Spring) 18, 384-390 (2010).

37. Devries, M.C., Samjoo, I.A., Hamadeh, M.J. \& Tarnopolsky, M.A. Effect of endurance exercise on hepatic lipid content, enzymes, and adiposity in men and women. Obesity (Silver Spring) 16, 2281-2288 (2008).

38. Shojaee-Moradie, F., et al. Exercise training reduces fatty acid availability and improves the insulin sensitivity of glucose metabolism. Diabetologia 50, 404-413 (2007).

39. Perseghin, G., et al. Increased glucose transport-phosphorylation and muscle glycogen synthesis after exercise training in insulin-resistant subjects. N Engl J Med 335, 1357-1362 (1996).

40. Egger, A., et al. The effect of aerobic exercise on intrahepatocellular and intramyocellular lipids in healthy subjects. PLoS One 8, e70865 (2013).

41. Bucher, J., et al. The effect of a single $2 \mathrm{~h}$ bout of aerobic exercise on ectopic lipids in skeletal muscle, liver and the myocardium. Diabetologia 57, 10011005 (2014).

42. Johnson, N.A., et al. Effect of prolonged exercise and pre-exercise dietary manipulation on hepatic triglycerides in trained men. Eur J Appl Physiol 112, 1817-1825 (2012).

43. Schrauwen, P., et al. Effect of acute exercise on uncoupling protein 3 is a fat metabolism-mediated effect. American journal of physiology. Endocrinology and metabolism 282, E11-17 (2002).

44. Szendroedi, J., et al. Abnormal hepatic energy homeostasis in type 2 diabetes. Hepatology 50, 1079-1086 (2009).

45. Schmid, A.l., et al. Liver ATP synthesis is lower and relates to insulin sensitivity in patients with type 2 diabetes. Diabetes Care 34, 448-453 (2011).

46. Cortez-Pinto, H., et al. Alterations in liver ATP homeostasis in human nonalcoholic steatohepatitis: a pilot study. JAMA 282, 1659-1664 (1999).

47. Camacho, R.C., Donahue, E.P., James, F.D., Berglund, E.D. \& Wasserman, D.H. Energy state of the liver during short-term and exhaustive exercise in 
C57BL/6J mice. American journal of physiology. Endocrinology and metabolism 290, E405-408 (2006).

48. Meyerhoff, D.J., Boska, M.D., Thomas, A.M. \& Weiner, M.W. Alcoholic liver disease: quantitative image-guided P-31 MR spectroscopy. Radiology 173, 393-400 (1989).

49. Snowling, N.J. \& Hopkins, W.G. Effects of different modes of exercise training on glucose control and risk factors for complications in type 2 diabetic patients: a meta-analysis. Diabetes Care 29, 2518-2527 (2006).

50. Stephens, N.A. \& Sparks, L.M. Resistance to the beneficial effects of exercise in type 2 diabetes: are some individuals programmed to fail? The Journal of clinical endocrinology and metabolism 100, 43-52 (2015).

51. Pandey, A., et al. Metabolic Effects of Exercise Training Among FitnessNonresponsive Patients With Type 2 Diabetes: The HART-D Study. Diabetes Care 38, 1494-1501 (2015).

52. Vollaard, N.B., et al. Systematic analysis of adaptations in aerobic capacity and submaximal energy metabolism provides a unique insight into determinants of human aerobic performance. J Appl Physiol (1985) 106, 1479-1486 (2009).

53. Brennan, A.M., Lam, M., Stotz, P., Hudson, R. \& Ross, R. Exercise-induced improvement in insulin sensitivity is not mediated by change in cardiorespiratory fitness. Diabetes Care 37, e95-97 (2014).

54. Cornelissen, V.A., Fagard, R.H., Coeckelberghs, E. \& Vanhees, L. Impact of resistance training on blood pressure and other cardiovascular risk factors: a meta-analysis of randomized, controlled trials. Hypertension 58, 950-958 (2011).

55. Pattyn, N., Cornelissen, V.A., Eshghi, S.R. \& Vanhees, L. The effect of exercise on the cardiovascular risk factors constituting the metabolic syndrome: a meta-analysis of controlled trials. Sports Med 43, 121-133 (2013).

56. Bouchard, C., et al. Adverse metabolic response to regular exercise: is it a rare or common occurrence? PLoS One 7, e37887 (2012).

57. Ramasamy, I. Recent advances in physiological lipoprotein metabolism. Clin Chem Lab Med 52, 1695-1727 (2014). 
58. Choi, S.H. \& Ginsberg, H.N. Increased very low density lipoprotein (VLDL) secretion, hepatic steatosis, and insulin resistance. Trends Endocrinol Metab 22, 353-363 (2011).

59. Eckardt, K., Gorgens, S.W., Raschke, S. \& Eckel, J. Myokines in insulin resistance and type 2 diabetes. Diabetologia 57, 1087-1099 (2014). 


ADDENDUM 



\section{SUMMARY}

The prevalence of obesity is reaching epidemic proportions worldwide. Environmental factors like consumption of high-caloric diets and low levels of physical activity are likely to underlie this increasing prevalence. Obesity is one of the most important risk factors contributing to mortality and the overall burden of disease. Type 2 diabetes mellitus (T2DM) may be the most devastating disease condition associated with obesity. T2DM is characterized by a gradual decline in insulin sensitivity, which impairs the effect of insulin and eventually elevates blood glucose values. The International Diabetes Federation (IDF) reported that 366 million people worldwide were suffering from diabetes in 2011. Ninety percent of those people suffered from T2DM, the most common form of diabetes. These number are likely to increase. The World Health Organization (WHO) predicts a 2fold increase in the prevalence of diabetes by 2030 .

The development of insulin resistance often coincides with fat accumulation in peripheral tissues. In the liver, this is referred to as non-alcoholic fatty liver (NAFL). NAFL develops when intrahepatic lipid (IHL) content exceeds $5 \%$ of the total liver weight in the absence of high alcohol consumption. Increased IHL content is associated with reduced hepatic, skeletal muscle and adipose tissue insulin sensitivity. Moreover, the risk to develop T2DM is higher in people with NAFL than in people without NAFL. In chapter 3, we compared the severity of metabolic disturbances in NAFL to those observed in T2DM. We found that in NAFL subjects hepatic, skeletal muscle and adipose tissue insulin sensitivity was similarly impaired as in T2DM patients. These observations pointed out that early diagnosis and proper treatment of insulin resistance in NAFL might be necessary to prevent development of T2DM.

Lifestyle interventions are considered a cornerstone for treatment of NAFL, and higher levels of physical activity are associated with lower levels of IHL content. Only a limited amount of interventional studies have investigated the effect of exercise training on IHL content, and most suggests that exercise training is mainly effective in lowering $\mathrm{IHL}$ content in people with high $\mathrm{IHL}$ content. While effects of exercise training might be long-term mediated, long-term effects of exercise 
training might also be explained by the accumulated effect of single exercise bouts. Therefore, in chapter 4, we were interested whether a single bout of exercise was also able to lower IHL content. However, no decrease in IHL content could be observed immediately or 4 hours post-exercise in men with overweight or NAFL. Instead, IHL increased 4 hours post-exercise, most likely due to the exerciseinduced elevation in plasma FFA levels.

We further explored the long-term effects of exercise training on hepatic lipid metabolism and insulin sensitivity in chapter 5. In this study people with and without NAFL and T2DM patients trained for 12 weeks, by combining aerobic and resistance exercise. Twelve weeks of supervised exercise training - without concomitant weight loss - lowered IHL content in subjects with NAFL. However, IHL content did not change in non-NAFL control subjects and T2DM patients. Remarkably, the exercise training induced decrease in IHL content in NAFL subjects was paralleled by improved skeletal muscle insulin sensitivity, but did not result in improved hepatic insulin sensitivity.

In chapter 6, we determined exercise training effects on adipose tissue gene expression of markers related to adipose tissue function in metabolically compromised and metabolically healthy obese subjects. While exercise training improved insulin sensitivity and maximal aerobic capacity, adipose tissue metabolism was not changed upon exercise training. Thus, exercise training mediated improvements in peripheral insulin sensitivity did not require adaptive responses in markers of subcutaneous white adipose tissue function in both, metabolically compromised and metabolically healthy obese subjects.

To detect the increase in cardiovascular risk that comes with obesity, the metabolic syndrome (MetS) has been defined as a constellation of cardiovascular risk factors. Like in T2DM and NAFL, management of the MetS includes recommendation of regular physical exercise. Thus, exercise training might also be an effective tool to improve the cardio-metabolic profile of overweight and obese subjects with MetS. In chapter 7 we found that 12 weeks of combined - aerobic and resistance - exercise training (CT) improved the metabolic profile of overweight and obese men with MetS. CT formally reduced the number of people with MetS as hypertension, one of the components contributing to MetS, did no longer exceed the cut-off level. 
Although the positive effect on the cardio-metabolic profile, CT did not improve cardiac function and arterial stiffness.

Despite the favorable effects of exercise training, not all individuals benefit from exercise training with respect to metabolic parameters. In a large group of overweight/obese control subjects and T2DM patients we explored possible differences in baseline parameters and evaluated the impact of exercise training on these parameters in responders (subjects who did increase insulin sensitivity upon exercise training) and non-responders (subjects who did not increase insulin sensitivity upon exercise training) (chapter 8). We were not able to identify any differences in baseline characteristics. Moreover, classical parameters of physical fitness and muscle strength increased similarly upon exercise training in responders and non-responders. Strikingly, fasting plasma glucose and triglyceride levels decreased in responders after exercise training, whereas both increased in nonresponders. Thus, a positive response on whole body insulin sensitivity upon exercise training seemed to be necessary to improve fasting plasma glucose and triglyceride levels.

In conclusion, the results obtained in this $\mathrm{PhD}$ thesis show that people with NAFL are characterized by severe insulin resistance, comparable to that seen in patients with T2DM. While acute exercise does not lowers IHL content, IHL content in people with NAFL decreases after exercise training, concomitant with an improvement in skeletal muscle insulin sensitivity. Exercise training does not improve markers of subcutaneous white adipose tissue function in metabolically compromised and metabolically healthy obese subjects, but is an effective treatment to decrease the cardio-metabolic risk in people with the metabolic syndrome. The divergent response in insulin sensitivity upon exercise training is paralleled by a different response in fasting plasma glucose and triglyceride concentrations. Further research is warranted to unravel the interrelationship between IHL content and insulin sensitivity, and to understand what causes the difference in response to exercise training in metabolically comparable people. 



\section{SAMENVATTING}

De wereldwijde prevalentie van obesitas bereikt epidemische proporties. Omgevingsfactoren - zoals de consumptie van calorierijke voeding en lage fysieke activiteit - liggen waarschijnlijk aan de oorzaak van deze toenemende prevalentie. Obesitas is een van de belangrijkste risicofactoren die bijdraagt tot het algemene sterftecijfer en de totale ziektelast. Type 2 diabetes mellitus (T2DM) is één van de ergste ziektes gekoppeld aan obesitas. T2DM wordt gekenmerkt door een geleidelijke daling in insulinegevoeligheid, waardoor het effect van insuline op het lichaam vermindert en uiteindelijk de bloedsuikerwaarden verhogen. Volgens de internationale Diabetes Federatie (IDF) leden er in 2011 wereldwijd 366 miljoen mensen aan diabetes. Negentig procent van deze 366 miljoen mensen leed aan T2DM, de meest voorkomende vorm van diabetes. Dit aantal mensen dreigt verder te verhogen. De Wereld GezondheidsOrganisatie (WHO) voorspelt een verdubbeling in de prevalentie van diabetes tegen 2030 .

De ontwikkeling van insulineresistentie valt vaak samen met vet accumulatie in perifere weefsels. In de lever wordt hiervoor de term niet-alcoholische vette lever (NAFL) gebruikt. NAFL ontwikkelt wanneer de intra-hepatische vet hoeveelheid (IHL) groter is dan $5 \%$ van het totale gewicht van de lever, in de afwezigheid van een verhoogde alcoholconsumptie. Een verhoogde IHL wordt geassocieerd met verminderde hepatische, skeletspier en vetweefsel insulinegevoeligheid. Bovendien is het risico voor het ontwikkelen van T2DM hoger in mensen met NAFL dan in mensen zonder NAFL. In hoofdstuk 3 vergeleken we de ernst van metabole storingen in NAFL met deze waargenomen in T2DM. We vonden dat hepatische, skeletspier en vetweefsel insulinegevoeligheid even erg verminderd was in NAFL proefpersonen dan in T2DM patiënten. Deze observaties wezen er op dat vroegtijdige diagnose en juiste behandeling van insulineresistentie in NAFL wellicht nodig is om de ontwikkeling van T2DM te voorkomen.

Verandering in levensstijl wordt beschouwd als een hoeksteen in de behandeling van NAFL, en een hogere fysieke activiteit worden geassocieerd met lagere niveaus van IHL. Slechts een beperkt aantal interventie studies hebben onderzocht wat het effect is van fysieke training op $\mathrm{IHL}$, en de meeste suggereren dat fysieke training 
vooral effectief is in het verlagen van IHL in mensen met een hoge vet hoeveelheid. Ondanks dat de effecten van fysieke training lange termijn gemedieerd kunnen zijn, kunnen de effecten van fysieke training misschien ook worden verklaard door het accumulerende effect van de aparte bewegingssessies. Daarom waren we in hoofdstuk 4 geïnteresseerd of een enkele bewegingssessie ook in staat was om de $\mathrm{IHL}$ te verlagen. We konden geen afname van de IHL waarnemen onmiddellijk of 4 uur na de bewegingssessie in mannen met overgewicht of NAFL. Daarentegen vonden we dat de IHL steeg 4 uur na de bewegingssessie. Deze observatie was hoogstwaarschijnlijk te wijten aan de verhoging van plasma vrije vetzuren veroorzaakt door de bewegingssessie.

Verder onderzochten we de lange termijn effecten van fysieke training op levervet metabolisme en insulinegevoeligheid in hoofdstuk $\mathbf{5}$. In deze studie werden mensen met en zonder NAFL en T2DM patiënten voor 12 weken getraind, door aerobe training en krachttraining te combineren. Twaalf weken fysieke training onder begeleiding - zonder gewichtsverlies - verlaagde de IHL in proefpersonen met NAFL. IHL veranderde echter niet in niet-NAFL controle proefpersonen en T2DM patiënten. Opmerkelijk, de afname in IHL door fysieke training in NAFL proefpersonen liep samen met een verbeterde insulinegevoeligheid in de skeletspieren, maar niet met een verbeterde hepatische insulinegevoeligheid.

In hoofdstuk 6 bepaalden we de effecten van fysieke training op genexpressie van markers gerelateerd aan vetweefsel functie in metabool gecompromitteerde en metabool gezonde proefpersonen met overgewicht en zwaarlijvigheid. Terwijl fysieke training de insulinegevoeligheid en maximale aerobe capaciteit verbeterde, was er geen effect op het vetweefsel metabolisme. Dus, de fysieke training gemedieerde verbeteringen in perifere insulinegevoeligheid waren niet afhankelijk van veranderingen in markers van vetweefsel functie, zowel bij metabool gecompromitteerde als bij metabool gezonde proefpersonen met overgewicht en zwaarlijvigheid.

Het metabool syndroom (MetS) is gedefinieerd als een verzameling van cardiovasculaire risicofactoren. Het MetS wordt gebruikt om de verhoging in cardiovasculair risico - gerelateerd met obesitas - te kunnen bepalen. Vergelijkbaar met T2DM en NAFL wordt regelmatige lichaamsbeweging ook aangeraden als 
behandeling van het MetS. Fysieke training zou dus ook doeltreffend kunnen zijn in het verbeteren van het cardio-metabole profiel van proefpersonen met MetS die overgewicht hebben of zwaarlijvig zijn. In hoofdstuk 7 vonden we dat 12 weken gecombineerde - aerobe en kracht - fysieke training (CT) het metabool profiel van mannen met het MetS, die overgewicht hebben of zwaarlijvig zijn, verbeterde. CT verminderde het aantal mensen die leden aan MetS duidelijk aangezien hypertensie - een van de onderdelen welke bijdraagt tot het MetS - niet langer de cutoff waarde overschreed. Ondanks het positieve effect op het cardio-metabole profiel, had CT geen positief effect op hartfunctie en arteriële vaatstijfheid.

Ondanks de gunstige effecten van fysieke training profiteren niet alle individuen van fysieke training met betrekking tot metabole parameters. In een grote groep van gezonde proefpersonen en T2DM patiënten met overgewicht en zwaarlijvigheid hebben we de mogelijke verschillen in karakteristieken onderzocht en hebben we de gevolgen van fysieke training op deze karakteristieken geëvalueerd in Responders (proefpersonen die hun insulinegevoeligheid na fysieke training verhogen) en non-Responders (proefpersonen die hun insulinegevoeligheid na fysieke training niet verhogen) (hoofdstuk 8). We konden geen verschillen observeren in de karakteristieken vóór de fysieke training interventie. Bovendien was de verbetering in klassieke parameters van fysieke fitness en spiersterkte na fysieke training in Responders en non-Responders vergelijkbaar. Opvallend was echter dat nuchtere glucose en triglyceride niveaus na fysieke training daalden in Responders. Daartegenover zagen we een stijging in beide parameters na fysieke training bij de non-Responders. Dus, een verbetering in insulinegevoeligheid na fysieke training leek nodig te zijn om de nuchtere glucose en triglyceride niveaus te verbeteren.

In conclusie tonen de resultaten in dit proefschrift aan dat mensen met NAFL worden gekenmerkt door ernstige insulineresistentie, vergelijkbaar met de insulineresistentie geobserveerd in patiënten met T2DM. Terwijl een enkele bewegingssessie IHL niet verlaagt, vermindert IHL bij mensen met NAFL na fysieke training. Daarbij treedt ook een verbetering van de skeletspier insulinegevoeligheid op. Fysieke training heeft geen effect op markers van vetweefsel functie bij metabool gecompromitteerde en metabool gezonde proefpersonen met overgewicht en zwaarlijvigheid. Het is wel een effectieve behandeling voor het 
verminderen van het cardio-metabole risico bij mensen met het metabool syndroom. De uiteenlopende reactie op insulinegevoeligheid na fysieke training loopt gelijk met een verschillende reactie op nuchtere glucose en triglyceride concentraties. Verder onderzoek is nodig om het onderlinge verband tussen IHL en insulinegevoeligheid te ontrafelen, en om de oorzaken van het verschil in reactie op fysieke training bij metabool vergelijkbare mensen beter te begrijpen. 




\section{VALORIZATION}

In this chapter we will cover (i) the social relevance, (ii) the target groups for whom this work could be interesting, (iii) the possible applications of our work for commercial activities, (iv) how innovative our results are, and (v) the future research possibilities.

\section{SOCIAL RELEVANCE}

The prevalence of obesity is very high and is reaching epidemic proportions worldwide. Obesity is one of the most important risk factor contributing to mortality and the overall burden of disease worldwide. The World Health Organization (WHO) describes obesity as one of the most blatantly visible, yet most neglected, public health problems that threatens to overwhelm both more and less developed countries. Since 1980, the obesity prevalence has nearly doubled, affecting 1.4 billion adults in 2008. Moreover, more than 40 million children under the age of 5 suffered from obesity.

Type 2 diabetes mellitus (T2DM) may be the most devastating disease condition associated with obesity. The International Diabetes Federation (IDF) reported that worldwide 366 million people were suffering from diabetes in 2011. Ninety percent of those people suffered from T2DM, the most common form of diabetes. The World Health Organization (WHO) predicts a 2-fold increase in the prevalence of diabetes by 2030 .

The development of obesity and T2DM coincides with fat accumulation in peripheral tissues. In the liver, this is referred to as non-alcoholic fatty liver (NAFL). NAFL is nowadays one of the most abundant liver diseases worldwide and has a very high prevalence in North and South America, much of Asia-Pacific, the Middle East, and Europe. The risk to develop T2DM is significantly higher in people with NAFL than in people without NAFL.

Environmental factors like consumption of high-caloric diets and low levels of physical activity are likely to underlie the increased prevalence in obesity, T2DM and NAFL. Because lack of exercise is considered an underlying cause, exercise training is considered a fundamental, important and cost-effective strategy for 
treatment and prevention. Current guidelines target 150 minutes of moderateintensity or 60 minutes of vigorous physical activity per week to prevent and treat obesity-related diseases.

\section{TARGET GROUPS}

The worldwide prevalence of obesity is very high and still increasing. As a consequence, the prevalence of obesity related comorbidities also increases. Interestingly, however, is the observation that not all people that are obese develop these comorbidities. Therefore, it is important to gather further understanding which underlying factors - related to obesity - contribute to decreased metabolic health. To answer this question, it is important to perform well-controlled clinical trials in human subjects.

In the first place, our obtained results will serve the scientific community via publications in international, peer-reviewed scientific journals. Publication of our observations will increase overall scientific knowledge and will be taken into account for future study proposals.

Secondly, our results might serve general physicians and medical doctors. In this thesis, we found that people with NAFL develop insulin resistance to a similar extend as T2DM patients. At present, people with NAFL are not treated for metabolic complications and therefore often develop T2DM later in life. Our results show that people with NAFL should be considered an important risk group to develop T2DM. Therefore, preventive treatment for metabolic complications in this population might be necessary. This knowledge might eventually be implemented in the clinic.

Exercise physiologist and lifestyle coaches are a third group of people that might use our results. Our work shows nicely that exercise training is very effective to treat obesity-related comorbidities, like insulin resistance, NAFL and the metabolic syndrome. Our results can contribute to improvements of the currently used exercise training protocols. Furthermore, we also show that - although people do improve their physical capacity with exercise training - some people do not improve their metabolic profile after exercise training. Thus, our findings might create a novel way to approach and measure exercise training effects. 


\section{ACTIVITIES AND PRODUCTS}

We found that people with NAFL develop insulin resistance to a similar extend as T2DM patients. Therefore, we suggested that it might be important to start treatment for metabolic complications already in people with NAFL. This suggested approach could serve as a potential novel healthcare strategy, that might reduce the amount of people that develop T2DM. We further found that when people with NAFL performed exercise training, the $\mathrm{IHL}$ content decreased and the insulin sensitivity increased, thus improving the metabolic profile. So, while today there is no treatment for people with NAFL regarding metabolic complications related to T2DM, our results show that exercise training might be an effective treatment to treat metabolic complications related to high IHL content.

Interestingly, we observed that - although people do improve their physical capacity with exercise training - some people do not improve their metabolic profile after exercise training. These findings might urge for a novel research field investigating the factors that determine exercise training effects in people.

All our results described in this thesis are documented in original scientific articles that have been published or are submitted to international scientific, wellrecognized, peer-reviewed journals. These articles can be found and accessed online by research scientists all over the world. Our results also have been presented on national and international conferences and can be used for future studies.

\section{INNOVATION}

The studies described in this thesis have been conducted at the department of human biology and human movement sciences of the Maastricht University Medical Center, which is known as a state of the art facility to conduct human research. Furthermore, this department holds a well-recognized scientific expertise in the field of obesity, type 2 diabetes, insulin resistance and exercise physiology research. The combination of well-recognized expertise and state of the art 
research facilities makes it possible to conduct innovative research that increases the knowledge in the scientific community.

All studies outlined in this thesis were performed in human volunteers. Although research in cells and rodents gives important insight in processes and pathways involved in certain disease conditions, it is of the uttermost importance to translate this research to humans. Not all research facilities have the opportunity and knowledge to perform this translational type of research. The department of human biology and human movement sciences of the Maastricht University Medical Center is one of the leading research facilities in the world performing translational research.

Most studies were done in collaboration with the department of radiology and endocrinology. These collaborations have been essential. The current non-invasive gold-standard to measure intrahepatic lipid (IHL) content is by using proton magnetic resonance spectroscopy $\left({ }^{1} \mathrm{H}-\mathrm{MRS}\right)$. These techniques were only available at the department of radiology. The collaboration with the department of endocrinology was necessary to elaborate the potential clinical implications of our study results.

All results described in this thesis are novel findings which have never been reported previously. Our results arise from close collaborations within the Maastricht University Medical Center, and from close collaborations with other Universities within the Netherlands and abroad.

\section{PLANNING AND REALIZATION}

In this thesis, clinical trials have been used to investigate the effects of exercise in NAFL and insulin resistance in humans. We demonstrated that exercise training is an effective treatment to lower IHL content in people with NAFL, improves insulin resistance, and improves the cardio-metabolic profile in people with the metabolic syndrome. We also observed that - although people do improve physical capacity with exercise training - some people do not improve their metabolic profile after exercise training. Further research is warranted to unravel the interrelationship between IHL content and insulin sensitivity, and to understand what causes the difference in response to exercise training in metabolically comparable people. The long-term collaboration between the department of human biology and human 
movement sciences with the department of radiology and the department of endocrinology will serve as a stable foundation to perform follow-up research in the research group of Prof. Dr. P. Schrauwen and Prof. Dr. M.K.C. Hesselink. 



\section{DANKWOORD}

Dit is het dan, met dit dankwoord sluit ik het hoofdstuk "promovendus" definitief af. Een periode van 5 jaar en een half leren, denken, hard werken, maar ook (zeker) veel plezier maken. Het was een periode waarin ik Maastricht leerde kennen als misschien wel de mooiste stad om in te wonen. Een periode waarin mijn Vlaamse accent vernederlandste. Een periode waarin ik pepernoten, Sateh-saus en bitterballen met mosterd ben gaan appreciëren, zo danig dat er momenten zijn waarop ik ze effectief mis.

Ik schrijf dit deel op zaterdagmiddag 26 maart 2016 - 2 dagen na mijn $30^{\mathrm{e}}$ verjaardag - buiten, met de Floridiaanse zon brandend op mijn gezicht. Ondanks kleine wetenschappelijk frustraties - omdat experimenten mislukten of proefpersoon inclusie niet altijd liep zoals ik het wou - heeft mijn prille wetenschappelijke carrière me nu reeds onvergetelijke momenten bezorgd. En niets van dit alles zou zijn gelukt zonder jullie steun, vertrouwen en vriendschap. Hierbij wil ik iedereen bedanken die ik op mijn pad ben tegen gekomen, met in het bijzonder volgende mensen.

Mijn promotoren en copromotor, Patrick, Matthijs en Vera. Zowel individueel als in team hebben jullie er aan bijgedragen dat ik nu een doctorsdiploma op zak heb. Dank jullie wel voor de mogelijkheden en de vrijheid die ik kreeg om zelf klinisch onderzoek uit te voeren, voor de dichte begeleiding in mijn ontwikkeling als wetenschapper en voor de mogelijkheid om wetenschappelijke congressen te bezoeken (en zo mooie steden te bezoeken onder het mom "werk"). Het laatste jaar was intens schrijven om het boekje snel af te krijgen. Ik ben dan ook dankbaar dat jullie de strikte deadlines respecteerden, zodat alles mooi op tijd is af geraakt!

Mijn lees- en beoordelingscommissie, Prof. Dr. Ir. A.M.W.J. Schols, Dr. M.R. Mensink, Prof. Dr. J. Plat, Prof. Dr. C.D.A. Stehouwer en Prof. Dr. S. R. Smith, with a special thank you for Prof. Dr. S.R. Smith, who gave me the opportunity to continue my scientific career in Orlando. I already enjoyed the first 6 months, but I am looking even more forward to the near future! 
Alle (ex)SHOCkers; jullie waren een geweldige groep om mee samen te werken. De SHOCk dagen waren altijd iets om naar uit te kijken!

Lena, from the start we were roomies and in those 5.5 years we never had any issues; we made a (the) strong(est) team. Although you prefer tea and I prefer coffee, we agreed on all other things; room temperature (basically, you could turn on the heating on 5 for the whole day, without me complaining;o)), office door open or closed, silence or music. I could not have had a better person to spend most hours of my day with. I may hope Eva is as good a roomie as I was ;o). Good luck with your postdoc, and your family life together with Stef and Sebastian.

Lauren, you and Heather are my first, and best, American friends. I am so happy that after 2 years Maastricht, I can work with you again in Orlando. Not only because you are a great friend, but also because you are a heck of a scientist! The 3 weeks I spend with you, Heather and Finley after my arrival in Orlando where amazing, a time I will never forget!

Tineke, als er 1 iemand een nog grotere mond heeft dan ik, dan ben jij het wel;o). Waarschijnlijk daarom dat we het zo goed met elkaar kunnen vinden. Jij was de perfecte gids tijdens mijn eerste maanden als AIO; om al het klinische werk onder de knie te krijgen en om mijn plaats te vinden in en groep met $90 \%$ vrouwen.

Esther P., jouw gedrevenheid en wetenschappelijke kennis heb ik altijd als een voorbeeld aanzien. Silvie, de Belgische connectie die met toch niet helemaal vreemd liet voelen de eerste dagen in Maastricht; veel succes in Nijmegen! Bianca, we konden goed lachen samen; geweldig dat je nu in Parijs werkt! Madeleen, veel respect voor jou als wetenschapper; na ons gesprek hier in Orlando ben ik maar al te blij dat je nu een mooie baan hebt dichter bij huis. Joris, de NVDO waren ieder jaar weer geweldig. Anne, Yvonne, Dennis, Lucas, Eline, Emmani, Esther K., Gert en Bas; dank jullie wel voor jullie helpende hand en expertise bij mijn experimenten. Marlies en Dirk, de buren; heerlijk om met een kop koffie binnen te wandelen en wat bij te praten. 
Alle andere HB-ers en BW-ers; twee mooie vakgroepen om voor te werken, met heel uitdagend en vernieuwend onderzoek.

Guy, we hebben en zullen nog mooie periodes beleven. Lange avonden met kaasen wijn, BBQ's, en in de Walkabout. Vaak waren wij de laatsten die nog aan de bar stonden bij promotiefeesten van anderen. Onze leuze luidt: Zolang het gezellig is en de bar niet sluit, ga je toch nog niet haar huis. Het was dan ook supermooi dat je in januari gewoon het vliegtuig nam, omdat het kon, en in Florida een week kwam "uitrusten". Voor jou staat de deur altijd open! Fantastisch dat jij mijn paranimf bent.

Boris, wat voor een ideeën hebben wij allemaal niet gehad de afgelopen jaren! We gingen de wereld veroveren met apps; soms "klooiden" we tot $4 u$ in de ochtend, met een pitstop rond $1 \mathrm{u}$ voor een Kapsalon. Avonden lang gezelschapspelletjes spelen - het liefste Le Havre - met een flesje wijn er bij en natuurlijk de "Famous Kingma Soup"; één ui, één courgette, één tomaat. Natuurlijk hebben we nog veel andere dingen meegemaakt; casino avonden waarbij we wonnen, casino avonden waarbij we alles opnieuw verloren. Het was dan ook heerlijk om hier onlangs in Orlando een weekend gezelschapspelletjes te spelen! Ik ben vereerd dat ook jij mijn paranimf bent, en natuurlijk staat ook voor jou de deur altijd open. Geniet van je gezinnetje, met Eva, Lisa, en de mooie uitbreiding die er zit aan te komen! Je bent een geweldige papa.

Maarten, veel van wat bij de vorige 2 heren staat kan ik natuurlijk copy-pasten, want vaak waren we gewoon met $z^{\prime} n$ allen op stap. Ik kan zeggen dat je een echte vriend bent. Je bent altijd oprecht, eerlijk, en je bent ontzettend verdraagzaam; dat kan ik met m'n hand op m'n hart zeggen na het kermis bezoek in München.. Je werkte hard als wetenschapper, maar nu heb je als ondernemer helemaal je ding gevonden; al had een gezamenlijke carrière bij de Lama's er ook in gezeten (herinner HB dagje uit)! Ik wens je van harte een prachtige toekomst binnen jouw familiebedrijf en samen met Sanne. Call me anytime!

Thomas, jij was de eerste collega die ik leerde kennen in Maastricht. Al werkten we niet in dezelfde vakgroep, tijdens de cursus PBL hadden we toch best een goeie tijd! Nadien werden onze wekelijkse koffies een begrip en een belangrijke routine. Wat een pad heb jij in tussentijd afgelegd; zelfstandige praktijk, getrouwd, huis gekocht, baby op komst, en hopelijk nog promoveren ook dit jaar! En dat terwijl ik je leerde kennen als een single kerel die in een studio woonde; Het leven is ook bij jou niet stil blijven staan! Wie weet wonen we binnen enkele jaren weer dichter bij elkaar, 
beiden professor in Maastricht ;o), en dan pikken we die wekelijkse koffies weer op!

Jasper, Masper, Most,.. Ik kan blijven doorgaan. Zelfde jaar geboren, zelfde jaar begonnen als AIO, zelfde jaar richting de VS, zelfde jaar gepromoveerd. Onze levens lopen zo parallel dat het maar goed is dat we vrienden zijn! Zowel als vriend en als wetenschapper heb ik ontzettend veel aan jou gehad. We konden uren praten over werk, voetbal, politiek, het onderwerp maakte eigenlijk niet uit. Zet ons samen aan een tafel, geef ons een lekker biertje, en we praten heel de avond vol. Ik hoop dat - tegen de tijd ik mijn proefschrift verdedig - ik jou een bezoek heb kunnen brengen in Baton Rouge om wat bij te praten. En mocht je niet bij mijn promotie aanwezig kunnen zijn - wat ik helemaal zou verstaan - dan vieren we binnenkort wel samen een Amerikaans feestje dat we nu eindelijk beiden officieel doctor zijn!

Stefan, de eerste 2.5 jaar beperkte onze band zich tot op het werk, maar wat hebben we daar de laatste 2.5 jaar verandering in gebracht! Ik kan je eerlijk zeggen, het was een fantastische keuze van jou om toch maar in Maastricht te komen wonen. De Walkabout, Van Bommel, De 2 Heren, Feesfebrik, Alla, noem ze maar op; we hebben ze allemaal meerdere keren bezocht. Je doet het nu prima in Singapore, en dat we nog steeds contact houden toont aan dat we in 2.5 jaar goede vrienden zijn geworden. Ik vind het doodzonde dat ik nog niet tot in Singapore ben geraakt. Maar hopelijk zien we elkaar ergens binnenkort, zodat ik de nieuwe mevrouw Camps te zien krijg;o).

Guy to the V(ijgen), je was een geweldige collega, met een efficiëntie die ik zelden heb gezien. Hopelijk tot binnenkort, en veel succes in Rotterdam met je werk en leven met Maartje. Erik (Kn)Aller, jou vergeten is onmogelijk, de spierbundel van $\mathrm{HB}$; wanneer ik op de $2^{\mathrm{e}}$ verdieping kwam viel er altijd wel iets te beleven in jouw buurt. Jos voor de heerlijke borrels. Antoine en Joan voor de hulp bij het prikken van een infuus. Loek en Paul voor hun Omnical- en computer-expertise. Gijs en Rudi voor de prettige samenwerking aan het adipose tissue paper. De secretaresses Desiree, Claudia en Yolanda voor de luchtige noot, behulpzaamheid en heerlijke pepernoten en paaseitjes. 
Mijn vrienden uit het Koninklijk Lyceum en de Universiteit Gent die de tand des tijds hebben doorstaan. Leendert, ik vergeet nooit onze rondreis in lerland en Noordlerland en onze stedentrip naar Barcelona, met de ochtendzon op ons terras achter de Plaza Catalunya. De pintjes in "het gildenhuis" vlak voor mijn vertrek naar de VS, waren zeker niet de laatste! Lode, onze stedentrips naar Lissabon, Madrid, Malta en de zomerweken dat je kwam "crashen" als mijn ouders op vakantie waren zijn prachtige herinneringen; jong en onbezonnen genieten van het goede leven (dat doen we nog steeds, natuurlijk). Ik heb het altijd ontzettend geapprecieerd dat jij me kwam bezoeken in Maastricht, om House of cards in 1 avond uit te kijken, of om FIFA te spelen tot in de vroege ochtend.

Elke, Mathieu en Tim; ondertussen kennen we elkaar al 12 jaar; tijd vliegt zeggen we dan. We zijn samen begonnen aan de Universiteit van Gent, van bachelor naar master in de biomedische wetenschappen. Ondertussen hebben we alle 4 onze eigen weg bewandeld; maar we vinden steeds de tijd om elkaar te zien, herinneringen op te halen en over de toekomst te praten. Het zegt veel dat we na al die jaren nog steeds vrienden zijn. Ik mag natuurlijk zeker Jonas, Carmen en Jolien niet vergeten; jullie 6 samen vormen één geheel dat me herinnert aan een prachtige studententijd!

Mijn familie; want dit zijn de meest belangrijke personen in iemand zijn leven. Familie is er altijd, goed of slecht, ver weg of dicht bij; onvoorwaardelijk.

Mijn ouders, mama en papa. Elk op jullie eigen manier hebben jullie er voor gezorgd dat ik nu sta waar ik sta. Jullie hebben mij getoond wat belangrijk is in het leven. Jullie hebben mij de kans en de vrijheid gegeven me te ontplooien. De afstand tussen ons is zeker niet altijd gemakkelijk, maar de band die we nu hebben is er één waar velen jaloers op zouden zijn.

Mijn broers, Jesse en Baptist. 6 handen op 1 buik, mijn beste vrienden. Jullie kunnen altijd op mij rekenen, net zoals ik altijd op jullie kan rekenen. Onze band is zoals een fles Romanée Conti; die was al goed in het begin, maar wordt nog beter met de jaren. Ik mis de zaterdagavonden aan het voetbalveld zeker, of de zondagavonden dat we samen F.C. de Kampioenen keken. Maar ik weet dat er ons nog veel mooie dingen te wachten staan. En natuurlijk ook Sofie, mijn schoonzus, zij die Jesse wat heeft weten temmen. 
Alle andere familie die hier vandaag speciaal aanwezig zijn; nonkels, ooms, tantes, neven en nichten; dank jullie wel voor de mooie momenten die we reeds samen hebben mogen meemaken.

Mijn schoonfamilie; Nathalie, Raf, Peter, Patricia, Myriam, Freddy en Linda. Dank jullie wel voor het vertrouwen dat jullie in mij stellen; je (klein)dochter meenemen naar de andere kant van de wereld is niet niets.

Tatiana, schat; reeds 6 jaar lang zit jij op de eerste rij. In al die tijd heb je mij gesteund, gestuurd, geholpen en vooral ook laten doen wanneer het nodig was; ik weet dat ik zeker niet altijd de gemakkelijkste ben. Dit boekje heeft veel tijd en opoffering van jou gevraagd; avonden en weekenden dat ik moest werken, en je eigenlijk niet veel aan me had. Maar dankzij jou staan we hier nu samen naast elkaar. Omdat ik al die tijd wist dat wanneer het even niet meer lukte, jij er voor me was. We wonen nu samen met Mila aan de andere kant van de wereld; ik had me geen beter leven kunnen voorstellen en ik ben super trots op wat jij ondertussen al hebt bereikt. Let's make some more memories.

Acta est fabula. 




\section{CURRICULUM VITAE}

Bram Brouwers was born on March 241986 in Ghent, Belgium. He graduated from secondary school at the Royal Lyceum Ghent in 2004 with a degree in mathematics and sciences. He joined Ghent University in September 2004 and obtained a Bachelor in Biomedical Sciences at Ghent University in August 2008. Subsequently, he joined the Master program in Biomedical Sciences at Ghent University. He graduated Cum Laude as a Master in Biomedical Sciences with a Major in Food and Metabolism in July 2010. The topic of his Master thesis was "The effect of rapamycin on angiogenesis in a diet-induced mouse model for non-alcoholic steatohepatitis".

Bram joined Maastricht University in The Netherlands in 2010 as a PhD student in the research group of Prof. Dr. P. Schrauwen and Prof. Dr. M.K.C. Hesselink. During his PhD trajectory he performed human intervention studies to investigate exercise effects on insulin resistance and liver, skeletal muscle and adipose tissue metabolism in relation to obesity, type 2 diabetes and non-alcoholic fatty liver disease.

In November 2015, Bram joined the research group of Prof. Dr. S.R. Smith at the Florida Hospital Translational Research Institute for Metabolism and Diabetes (TRI$\mathrm{MD}$ ) in Orlando, Florida, USA. During his Postdoctoral research he will further investigate the metabolic complications observed in obesity and type 2 diabetes. 



\section{LIST OF PUBLICATIONS}

B Brouwers, MKC Hesselink, P Schrauwen, VB Schrauwen-Hinderling. Effects of exercise training on intrahepatic lipid content in humans. Submitted

B Brouwers, VB Schrauwen-Hinderling, T Jelenik, A Gemmink, B Havekes, Y Bruls, D Dahlmans, M Roden, MKC Hesselink, $\mathrm{P}$ Schrauwen. Exercise training reduces liver fat content and improves peripheral but not hepatic insulin sensitivity in overweight and obese men with non-alcoholic fatty liver disease. Submitted

B Brouwers, R Stinkens, JWE Jocken, EE Blaak, MKC Hesselink, P Schrauwen, G Goossens. Exercise training-induced effects on abdominal subcutaneous adipose tissue gene expression in obese humans. In prep

B Brouwers, PJ Joris, MKC Hesselink, P Schrauwen, VB Schrauwen-Hinderling. Combined aerobic and resistance exercise training improves the cardio-metabolic profile in overweight and obese men with metabolic syndrome. In prep

B Brouwers, RCM Meex, VB Schrauwen-Hinderling, E Phielix, B Havekes, P Schrauwen, MKC Hesselink. The dual faces of exercise training on whole body insulin sensitivity. In prep

B Brouwers, L Bilet, PA van Ewijk, MKC Hesselink, ME Kooi, P Schrauwen, VB Schrauwen-Hinderling. Acute exercise does not decrease liver fat in men with overweight or NAFLD. Scientific reports; 2015 Apr, 13;5:9709

L Lindeboom, Cl Nabuurs, J Hoeks, B Brouwers, E Phielix, ME Kooi, MKC Hesselink, JE Wildberger, RD Stevens, T Koves, DM Muoio, P Schrauwen, VB SchrauwenHinderling. Long-echo time MR spectroscopy for skeletal muscle acetylcarnitine detection. Journal of Clinical Investigation; 2014 Nov;124(11):4915-25 
LM Sparks, M Bosma, B Brouwers, T van de Weijer, L Bilet, G Schaart, E MoonenKornips, TO Eichmann, A Lass, MKC Hesselink, P Schrauwen. Reduced incorporation of fatty acids into triacylglycerol in myotubes from obese individuals with type 2 diabetes. Diabetes. 2014 May;63(5):1583-93 